Universidade de São Paulo

Instituto de Astronomia, Geofísica e Ciências Atmosféricas

Departamento de Astronomia

Thaíse da Silva Rodrigues

\title{
Determinação da Distribuição de Idades de Estrelas Centrais de Nebulosas Planetárias
}

São Paulo 

Thaíse da Silva Rodrigues

\section{Determinação da Distribuição de Idades de Estrelas Centrais de Nebulosas Planetárias}

Dissertação apresentada ao Departamento de Astronomia do Instituto de Astronomia, Geofísica

e Ciências Atmosféricas da Universidade de São Paulo como requisito parcial para a obtenção do título de Mestre em Ciências.

Versão corrigida. Versão original encontra-se na unidade.

Área de Concentração: Astronomia

Orientador: Prof. Dr. Walter Junqueira Maciel

São Paulo 

À minha família: Lecir, Rubens, Diego e Matheus 



\section{Agradecimentos}

Em um determinado momento durante o mestrado, me deparei fazendo uma retrospectiva do porquê escolhi fazer astronomia. Resolvi então escrever um pouco sobre isso, para que as pessoas que aqui citarei percebam nas entrelinhas o quanto foram importantes durante todo meu trajeto.

Minha admiração pela astronomia começou pelas revistas de divulgação científica que meus pais, Lecir e Rubens, e minha tia Wanda me presentearam, tornando-se um desejo ao assistir as aulas de física ministradas com entusiasmo pelo professor Eduardo durante o ensino médio. Quando decidi prestar o vestibular para Astronomia, meus pais, apesar de apreensivos, me deram todo o apoio necessário, emocional e financeiramente. Enquanto minha avó Cenira pedia em suas orações para que eu mudasse de idéia, pois não queria que eu fosse morar na "cidade grande" (risos!). Ela não pôde imaginar o quanto seus ensinamentos me prepararam para isso! O apoio de amigos, como o da Liliana, Natália, Roberta e Victor, foram essenciais. Nesse momento, conheci alguém que foi um porto seguro em todos esses anos que se passaram: Juan Pablo, muito mais que um amigo.

Para conhecer um pouco do que eu pretendia seguir, troquei e-mails com um profissional da área que me esclareceu vários pontos (positivos e negativos) sobre o curso e a profissão. Recentemente descobri que quem fez questão de tirar minhas dúvidas é o professor Roberto Costa, o qual foi professor de uma das minhas máterias no mestrado. Escolhi fazer o curso de Astronomia no OV/UFRJ, embora tivesse a opção de fazer o curso de Física na UFMS e ficar mais perto de casa. Minha vida mudou totalmente, pois aos 17 anos eu saí do interior para correr atrás do que eu denominava "meu sonho". Cada passo que dei foi um desafio, tudo era completamente novo.

Com o passar do tempo, percebi que os cursos de exatas não selecionam os mais "in- 
teligentes", e sim, os que têm mais capacidade de resistir. Acompanhei vários amigos e colegas desistindo daquele "sonho em comum" e me vi desistindo de várias outras coisas para seguir em frente. Todos passam por isso um dia (ou vários!). Os amigos Ihani, João Antônio, Loloano, Luana, Lygia e Victor me ajudaram tanto nesses momentos de dúvida quanto nos feriados longos e nas férias em que eu não podia viajar para rever minha família, nos finais de semana e noites viradas estudando, e nos momentos de descontração: assistindo jogo de futebol, desabafando em algum boteco ou simplesmente conversando. Além disso, meus irmãos, mesmo distantes, foram importantes. O Diego por se tornar cada vez mais um amigo, e o Matheus pelas incessantes perguntas sobre tudo, achando que eu sabia a resposta para todos os porquês, e pela sua ingenuidade em dizer que minhas explicações sempre estavam erradas por mais que fossem comprovadas fisicamente!

Desde o primeiro semestre, percebi que a área que mais me encantava era Astrofísica Estelar. Meu primeiro passo nessa direção foi a iniciação científica com o professor Gustavo Porto de Mello, que foi um ótimo orientador, professor e amigo, sempre alegre, disposto e apaixonado pelo que fazia e faz. Outros professores fundamentais para minha formação foram Armando Aleixo, Belita Koiller, Luiz Felipe Coelho e Sergio Joraz do IF/UFRJ; Denise Gonçalves e Helio Rocha Pinto do OV/UFRJ. Todos transmitiram o conhecimento que obtiveram da melhor forma possível.

No fim da graduação, tive outra grande dúvida: o mestrado. Decidi vir para o IAG/USP, o que envolveu deixar tudo que havia construído no Rio de Janeiro e carregar todas as bagagens tanto físicas, como todo o material adquirido ao longo da graduação, quanto emocionais, como as experiências e maturidade, para recomeçar em um lugar novo. Mais uma vez, cada passo foi um desafio. Meus pais e toda minha família novamente me apoiaram. Juan Pablo me deu forças para buscar o que eu queria. Desde o início pude contar com minhas primas Marcia e Elenay. Ganhei um lar na casa da Marcia com seu filho, João Pedro, que alegra meus dias, e uma família "bem" grande com a Elenay, Hélio, Luiz Felipe, Yanele e Daniel.

No IAG, tive o prazer de ter como orientador o professor Walter Maciel. Ele ministrou com extrema dedicação duas matérias importantes para minha formação, e também me auxiliou em todos os aspectos necessários durante o mestrado. Agradeço pela oportunidade que tive em desenvolver um projeto orientado por ele, e por todo o conhecimento que me 
transmitiu. Entre as pessoas que conheci nesse instituto, construí uma amizade especial com a Aiara, minha Florzinha. Aprendemos tantas coisas uma com a outra, que parece que somos amigas de infância. Um outro amigo importante que fiz é o Daniel, Dani Dani! Foram tantas palavras trocadas, com ou sem nexo, que me possibilitaram ver as coisas por vários outros ângulos! Os dois foram mais que importantes para que eu chegasse "sã" nesse ponto da minha carreira (risos!). Além disso, o trio Marcia, Yanele e Bárbara foram essenciais para me manter firme na minha vida pessoal: amigas, psicólogas, "sócias", companheiras de balada. Não posso deixar de dizer que Aiara, Bárbara, Daniel, Juan Pablo e Yanele foram fundamentais para que essa dissertação desabrochasse!

Quero agradecer também aos colegas de sala: Guilherme, Graziela e Rodrigo; aos amigos da pós-graduação: Cesar, Juan Carlos, Luciene, Marcio, Nadili, Rafael, Reinaldo e Silvio; aos meus tios e tias, primos e primas, aos funcionários do IAG, aos paistrocínio e tiostrocínio. A todos vocês o meu "muito obrigada". Se estou aqui hoje é porque cada um de vocês me possibilitou construir um pouco da longa estrada que percorri.

Este trabalho foi financiado pelas agências CNPq (processo 133151/2010-7) e FAPESP (processos 2006/59453-0 e 2010/18835-3).

Esta dissertação foi escrita em AATEX com a classe IAGTESE, para teses e dissertações do IAG. 

"Tudo no mundo começou com um sim. Uma molécula disse sim a outra molécula e nasceu a vida. Mas antes da pré-história havia a pré-história da pré-história e havia o nunca e havia o sim. Sempre houve. Não sei o quê, mas sei que o universo jamais começou. Que ninguém se engane, só consigo a simplicidade através de muito trabalho. Enquanto eu tiver perguntas e não houver resposta continuarei a escrever."

A Hora da Estrela - Clarice Lispector

"... não conhece nada mais belo do que partir para o desconhecido."

A Insustentável Leveza do Ser - Milan Kundera 



\section{Resumo}

Estrelas centrais de nebulosas planetárias (ECNPs) têm um intervalo de massa relativamente grande na fase da sequência principal, por isso espera-se que elas também tenham diferentes idades, tipicamente acima de 1 Gano. Além de necessárias para o conhecimento das propriedades das ECNPs, a determinação das idades é também importante no contexto da evolução química da Galáxia, como no estudo da variação temporal dos gradientes radiais de abundâncias químicas. Entretanto, não há um método único e confiável que possa ser aplicado para estimar a idade de todos os tipos de estrelas. Neste projeto, desenvolvemos dois métodos de idades cinemáticas baseados na relação idade-dispersão de velocidade do disco da Galáxia encontrada pelo recente levantamento Geneva-Copenhagen. O primeiro método compara a velocidade de rotação esperada da ECNP com a velocidade predita pela curva de rotação da Galáxia em sua posição, e interpreta a diferença entre elas como a dispersão de velocidade do objeto, relacionada com a idade. O segundo método consiste em calcular as componentes da velocidade espacial, com algumas hipóteses, e estimar diretamente as dispersões das velocidades, inferindo uma idade pela relação adotada. Duas amostras foram escolhidas, contendo 234 e 866 nebulosas planetárias, para as quais velocidades radiais precisas estão disponíveis na literatura. Os resultados sugerem que a maioria das ECNPs no disco Galáctico tem idade abaixo de 5 Gano, e um pico ente 0 e 3 Gano. Esses resultados são comparados com algumas distribuições recentes de idades baseadas em correlações independentes envolvendo as abundâncias químicas das nebulosas planetárias. 



\section{Abstract}

Central stars of planetary nebulae (CSPN) have a relatively large mass interval on the main sequence, so that it is expected that these stars also have different ages, typically above 1 Gyr. Apart from the properties of the CSPN themselves, the problem of age determination is also important in the context of the chemical evolution of the Galaxy, as in the understanding of the time variation of chemical abundance gradients. However, there are no unique and reliable methods that can be applied to estimate the age of all types of stars. In this work, we developed two methods of kinematic ages based on the age-velocity dispersion relation of the Galactic disk derived by the recent GenevaCopenhagen survey. The first method compares the expected rotation velocity of CSPN with the predicted velocity by the Galactic rotation curve at its position, and interprets the difference between them as the velocity dispersion of the star, which is related to the stellar age. The second method consists in calculating the U, V, W velocity components of CSPN, with some hypotheses, and estimating directly the velocity dispersions, so that the age can be derived. Two samples were chosen, containing 234 and 866 nebulae, for which accurate radial velocities are available in the literature. The results suggest the most CSPN in the Galactic disk have ages under 5 Gyr with a distribution peaked between 0 and 3 Gyr. These results are also compared with some recent age distributions based on independent correlations involving the nebular chemical abundances. 



\section{Lista de Figuras}

$1.1 \quad$ Diagrama $\mathrm{HR}$ de uma estrela de $3 \mathrm{M}_{\odot}$. . . . . . . . . . . . . . . . . . . . . 23

2.1 Posição Galáctica dos objetos das amostras. . . . . . . . . . . . . . . . . . 37

2.2 Distribuições de distâncias heliocêntricas obtidas do catálogo de Maciel (1984). 40

2.3 Distribuiçoes de distâncias heliocêntricas obtidas do catálogo de Stanghellini et al. (2008). . . . . . . . . . . . . . . . . . . . 4 41

2.4 Distâncias de Stanghellini et al. (2008) versus distâncias de Maciel (1984). $\quad 42$

3.1 Relação idade-dispersão de velocidade do levantamento Geneva-Copenhagen. 49

3.2 Curvas de rotação Galáctica de Clemens (1985) e Maciel e Lago (2005). . 55

4.1 Distribuições de distâncias Galactocêntricas. . . . . . . . . . . . . . . . . . 60

4.2 Distribuições de idades das ECNPs das Amostras A e B, método 1. . . . . 62

4.3 Distribuiçoes das velocidades $U, V, W$ e $T$ das Amostras A e B, método 2 , hipótese do movimento próprio nulo . . . . . . . . . . . . . . . . . . . . . 65

4.4 Distribuições de idades das ECNPs das Amostras A e B, método 2, hipótese do movimento próprio nulo. . . . . . . . . . . . . . . . . . 67

4.5 Distribuiçoes das velocidades $U, V, W$ e $T$ das Amostras A e B, método 2 , hipótese do movimento próprio não-nulo. . . . . . . . . . . . . . . . . . . . 71

4.6 Distribuições de idades das ECNPs das Amostras A e B, método 2, hipótese do movimento próprio não-nulo . . . . . . . . . . . . . . . . . . 73

4.7 Representação gráfica de um boxplot. . . . . . . . . . . . . . . . . . . . . . 75

4.8 Distribuiçóes de idades das ECNPs da Amostra B, método 2, hipótese do movimento próprio não-nulo com a variação de seu sinal. . . . . . . . . . . 76 
4.9 Distribuiçoes de idades das ECNPs da Amostra B, método 2, hipótese do movimento próprio nulo, limite inferior na relação idade-dispersão de velocidade . . . . . . . . . . . . . . . . . . . . . . . 80

4.10 Distribuições de idades das ECNPs da Amostra B, método 2, hipótese do movimento próprio não-nulo, limite inferior na relação idade-dispersão de velocidade . . . . . . . . . . . . . . . . . . . . . 81

4.11 Distribuições das velocidades $U, V, W$ e $T$ das ECNPs com determinações

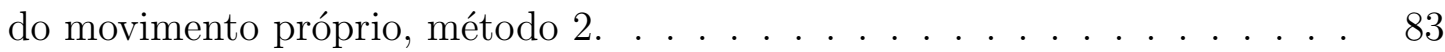

4.12 Distribuições de idades das ECNPs com determinaçoes do movimento próprio,

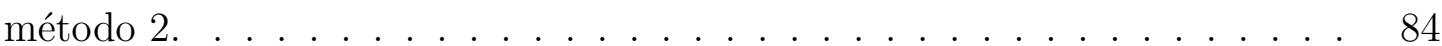

4.13 Distribuição do módulo da velocidade radial e módulo da velocidade tangencial versus módulo da velocidade radial. . . . . . . . . . . . . . . . . 85 


\section{Lista de Tabelas}

1.1 Classificação de Peimbert, massa da progenitora da NP e estimativa da idade. 29

2.1 Classificação de Peimbert das amostras. . . . . . . . . . . . . . . . . . . . 36

3.1 Coeficientes da relação idade-dispersão de velocidade. . . . . . . . . . . . . 50

3.2 Coeficientes do polinômio da curva de rotação Galáctica de Clemens (1985). 54

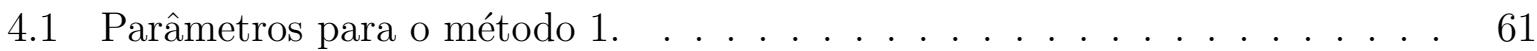

4.2 Média aritmética das velocidades com a hipótese do movimento próprio nulo. 66

4.3 Parâmetros para o método 2 com a hipótese do movimento próprio nulo. . 68

4.4 Média aritmética das velocidades com a hipótese do movimento próprio não-nulo . . . . . . . . . . . . . . . . . . . . . . . . 70

4.5 Parâmetros para o método 2 com a hipótese do movimento próprio não-nulo. 72

A.1 Amostra de nebulosas planetárias. . . . . . . . . . . . . . . . . . . . . . 101

B.1 Amostra de nebulosas planetárias com determinações do movimento próprio. 123 



\section{Sumário}

1. Introduçãd . . . . . . . . . . . . . . . . . . . . . . . . 21

1.1 Nebulosas Planetárias e suas Estrelas Centrais . . . . . . . . . . . . . . . . 22

L.2 ddades de Estrelas . . . . . . . . . . . . . . . . . . . . . . . . . . . . . . 24

T.2.1 Métodos de Éstimar ldades . . . . . . . . . . . . . . . . 25

1.22 Métodos de Estimar ldades de ECNPS . . . . . . . . . . . . . 27

1.2.2.1 Classificação de Peimbert . . . . . . . . . . . . . . . 28

1.2 .2 .2 Relação Tdade-Metalicidade . . . . . . . . . . . . . . . . . 30

$1.2 .2 .3 \quad$ Relação Idade-Massa (N/O) . . . . . . . . . . . . . . . . . 31

1.2 .2 .4 Distribuições de Massa Empíricas . . . . . . . . . . . . . . 32

[2.2.5 lsócronas . . . . . . . . . . . . . . . 33

1.3 Objetivo do Trabalho . . . . . . . . . . . . . . . . . . . . . . . . . 34

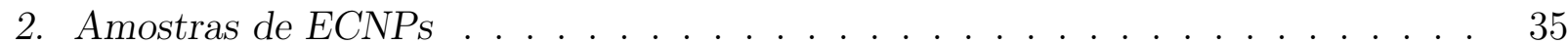

2.1 Introdução . . . . . . . . . . . . . . . . . . . . . . . . 35

2.2 Distâncias Heliocêntricas . . . . . . . . . . . . . . . . . . . . 36

2.2 .1 Éscala de Distância de Maciel e Pottasch . . . . . . . . . . . . . . . 38

2.2 .2 Escala de Distância de Stanghellini, Shaw e Villaver . . . . . . . . . 39

2.3 Velocidades Radiais Heliocêntricas . . . . . . . . . . . . . . . . . . . . . 42

3. Idades Cinemáticas . . . . . . . . . . . . . . . . . . . . 45

3.1 Relação Idade-Dispersão de Velocidade . . . . . . . . . . . . . . . . . . . . 45

3.1 .1 Processos de Aquecimento do Disco . . . . . . . . . . . . . . . . . . 46

3.1 .2 Levantamento Geneva-Copenhagen . . . . . . . . . . . . . . . . . . 48 
3.2 Métodos de Estimar dades Cinemáticas . . . . . . . . . . . . . . 51

3.2.1 Método 1: Velocidade de Rotacão das NPs . . . . . . . . . . . . 52

3.2.1.1 Curvas de Rotação Galácticas . . . . . . . . . . . . . . 53

3.2.2 Método 2: Componentes U, V e $\mathrm{W}$ da Velocidade Éspacial . . . . . 55

4. Resultados e Discussãd . . . . . . . . . . . . . . . . . . . . . . . . . . . . . . . . 59

4.1 Distribuiçóes de Idades Cinemáticas: Método 1 $\ldots$. . . . . . . . . . . . . . 59

4.2 Distribuiçoes de Tdades Cinemáticas: Método 2 . . . . . . . . . . . . . . . 63

4.2 .1 Movimento Próprio Nulo . . . . . . . . . . . . . . . . . . 64

4.2 .2 Movimento Próprio Não-Nulo $\ldots \ldots \ldots$. . . . . . . . . . . . . . . . . 68

4.2 .3 Teste do Sinal do Movimento Próprio . . . . . . . . . . . . . . . . . 74

4.2 .4 Limites da Relação Tdade-Dispersão de Velocidade . . . . . . . . . . 77

4.2 .5 Objetos com Determinaçoes do Movimento Próprio . . . . . . . . . 79

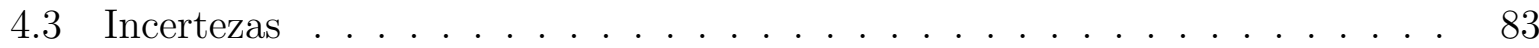

5. Conclusões e Perspectivas . . . . . . . . . . . . . . . . . . . . . 87

5.1 Conclusones . . . . . . . . . . . . . . . . . . . . . . . . . . . . . . . . 87

5.2 Perspectivas Futuras $\ldots \ldots \ldots \ldots$

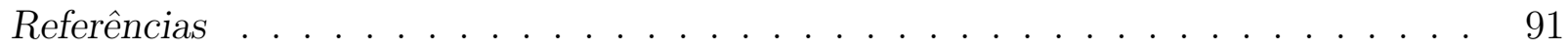

$\begin{array}{ll}\text { Apêndice } & 99\end{array}$

A. Amostra de Nebulosas Planetárias . . . . . . . . . . . . . . . . . . . 101

B. NPS com Determinaçôes do Movimento Próprid . . . . . . . . . . . . . . . . . . 123

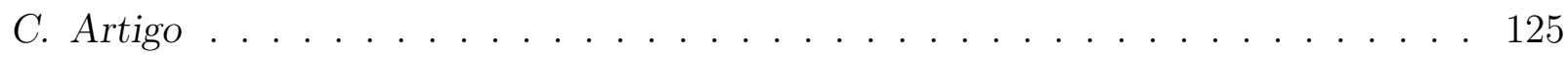


Capítulo 1

\section{Introdução}

A estrutura e a evolução de uma estrela podem ser inferidas se sua massa e composição química forem conhecidas, segundo o teorema de Vogt-Russell. Propriedades físicas, tais como campo magnético, rotação, entre outras, influenciam o interior estelar, porém em menor escala. A própria composição química da estrela modifica-se através das fusões de elementos químicos que ocorrem em seu interior com o tempo, logo, é necessário considerar a idade como um fator importante na evolução, apesar de não ser o agente causador dessas mudanças. Como a massa é a propriedade dominante, as estrelas são divididas em grupos de massa baixa, intermediária e alta, conforme as reações nucleares que ocorrem em seu interior. Todas as estrelas passam a maior parte de suas vidas na chamada sequência principal, cerca de 90\%, período no qual ocorre a fusão de hidrogênio em hélio em seus núcleos, porém a evolução de cada grupo ocorre de forma diferente, resultando objetos finais distintos.

As estrelas com massas muito baixas, menores que 0,5 massas solares $\left(\mathrm{M}_{\odot}\right)$ evoluem para anãs brancas de hélio. As estrelas de massa baixa e intermediária, após ejetarem seus envoltórios em forma de nebulosas planetárias, resultam em anãs brancas de carbono e oxigênio. As estrelas massivas $\left(>8 \mathrm{M}_{\odot}\right)$ sofrem o colapso de seus núcleos e a consequente explosão em supernova, resultando em uma estrela de nêutron ou em um buraco negro. Uma descrição detalhada da evolução desses grupos de estrelas não é relevante para esta dissertação, por essa razão iremos nos concentrar apenas nas estrelas de massa baixa e intermediária, em suas fases de nebulosas planetárias. Detalhes sobre a evolução estelar, principalmente de estrelas de massa baixa e intermediária, podem ser encontrados em Kippenhahn e Weigert (1994) e Maciel (14999). 


\subsection{Nebulosas Planetárias e suas Estrelas Centrais}

Após a exaustão de hidrogênio em seu núcleo, a estrela de massa baixa $(\approx 0,8$ a 2,5 $\mathrm{M}_{\odot}$ ) deixa a sequência principal (SP) e caminha pelo ramo das sub-gigantes no diagrama Hertzsprung-Russell (HR). Seu núcleo começa a contrair e a queima de hidrogênio continua em uma espessa camada ao redor do núcleo. O envoltório da estrela se aquece e expande, sua temperatura efetiva decai e a estrela entra no ramo das gigantes vermelhas (RGB, sigla do inglês: red giant branch). Sua densidade central fica tão elevada que o gás de elétrons torna-se degenerado. Quando a estrela atinge o topo do ramo das gigantes vermelhas, sua temperatura central está alta o suficiente para dar início à queima de hélio, chamado flash do hélio. Então a estrela passa para o ramo horizontal no diagrama HR diminuindo seu raio e aumentando sua temperatura efetiva.

Quando a fusão do hélio cessa no interior estelar, o núcleo novamente contrai e o envoltório se expande, aumentando o raio e a luminosidade, e a estrela entra no ramo assintótico das gigantes (AGB, sigla do inglês: asymptotic giant branch). Novamente o gás de elétrons do núcleo de carbono-oxigênio torna-se degenerado, e nessa fase a estrela possui duas camadas de queima: uma após o núcleo (de hélio) e outra mais externa (de hidrogênio). Durante esse estágio, a estrela perde massa por ventos, apresentando posteriormente ventos mais intensos que levam à ejeção das suas camadas mais externas na forma de nebulosa planetária (NP). O produto restante é o seu núcleo, quente e brilhante, chamado de estrela central de nebulosa planetária (ECNP), que ioniza o material ejetado. A ECNP caminha para temperaturas mais altas no diagrama HR mantendo a luminosidade aproximadamente constante. Com o fim das reações nucleares, a ECNP torna-se uma anã branca (WD, sigla do inglês: white dwarf) e resfria-se, perdendo sua luminosidade. Os restos da nebulosa planetária se misturam com o material do meio interestelar, sendo futuramente utilizados na formação de novas estrelas.

As estrelas de massa intermediária $\left(\approx 2,5\right.$ a $\left.8 \mathrm{M}_{\odot}\right)$ passam por uma evolução semelhante às de massa baixa, porém não iniciam a queima do hélio com um núcleo de gás de elétrons degenerado. A figura [L], retirada de Blöcker ([200)]), apresenta o diagrama HR para uma estrela de $3 \mathrm{M}_{\odot}$ e suas fases evolutivas. A escala de tempo dessas estrelas na sequência principal é da ordem de $10^{9}$ anos (1 Gano), na fase do ramo assintótico das gigantes em torno de $10^{6}$ anos, e na fase de nebulosa planetária, $10^{4}$ anos. 


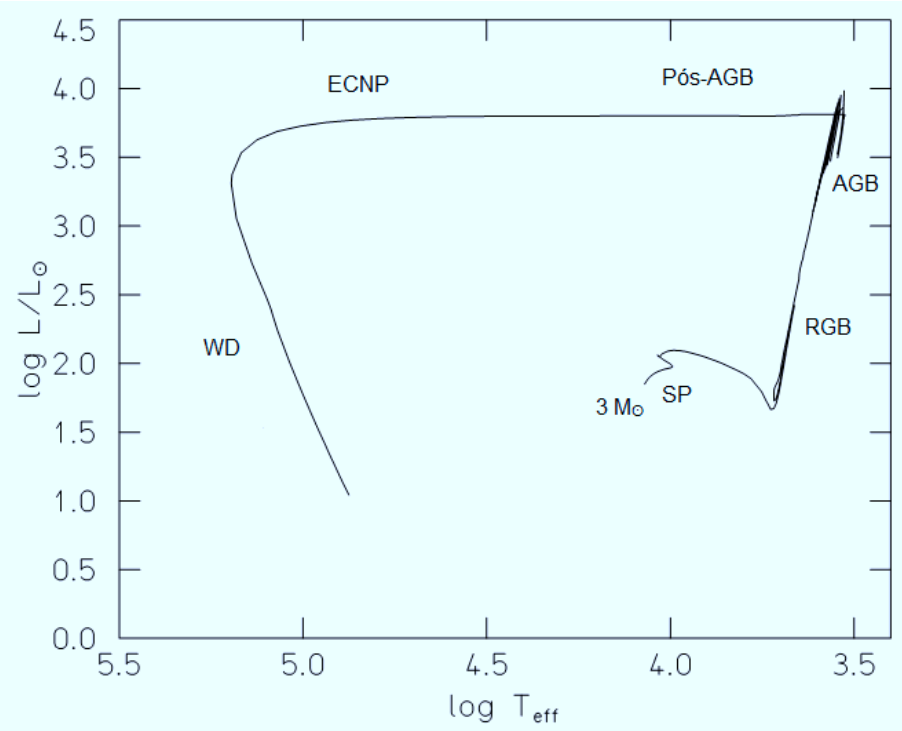

Figura 1.1: Diagrama HR de uma estrela de $3 \mathrm{M}_{\odot}$ e suas fases evolutivas. Figura retirada de Blöcker (200) .

As nebulosas planetárias são nebulosas difusas que consistem basicamente de um envoltório gasoso, contendo hidrogênio, hélio, carbono, oxigênio, e outros elementos pesados, com uma temperatura eletrônica da ordem de $10^{4}$ kelvin e densidades típicas de $10^{4}$ partículas por centímetro cúbico. São objetos interessantes, uma vez que pode-se determinar a abundância de elementos químicos leves e sintetizados pelos processos de nucleossíntese, como hélio, carbono e nitrogênio, e também de elementos mais pesados e que seriam representantes do meio interestelar onde a estrela progenitora se formou, tais como oxigênio, neônio, entre outros.

As ECNPs, na maioria das vezes, não são observadas diretamente e possuem temperaturas altas (em torno de $10^{5} \mathrm{~K}$ ), emitindo parte de seu fluxo fora do espectro visível. Suas massas distribuem-se entre 0,5 e $1,2 \mathrm{M}_{\odot}$, com uma média em torno de 0,6 a $0,8 \mathrm{M}_{\odot}$. Devido ao intervalo relativamente extenso de massas na fase da sequência principal, espera-se que essas estrelas tenham também idades diferentes, tipicamente acima de 1 Gano, representando tanto uma população de estrelas relativamente jovens (mais massivas) quanto de idades mais avançadas (massa baixa). Por essas razões, nebulosas planetárias são objetos atraentes para o estudo da evolução química da Galáxia, pois além de fornecerem abundâncias de elementos químicos com uma boa precisão, estão distribuídas pela Galáxia e suas estrelas centrais podem ter uma ampla variação de idade. Dessa forma, NPs podem 
contribuir para investigar a evolução química e dinâmica da Galáxia juntamente com as regiões $\mathrm{H}$ II, estrelas de tipo espectral $\mathrm{O}$ e B, entre outros objetos.

O conhecimento de tais características das NPs é particularmente relevante para o estudo de gradientes radiais de abundâncias e de suas variações com o tempo no disco da Galáxia, os quais são vínculos importantes para os modelos de evolução química. Vários trabalhos têm determinado gradientes de abundâncias radiais utilizando nebulosas planetárias (Maciel e Köppen, 1994; Maciel e Quireza, 19999), e há diversas evidências que indicam uma variação temporal desses gradientes (Maciel et al, 2003, 2005, 2006). Portanto, é necessário ter, além de abundâncias e distâncias bem determinadas, o conhecimento adequado das idades das ECNPs. A determinação da idade de uma estrela é um problema complexo, não havendo para isso um método único e confiável, o que será descrito na próxima seção.

\subsection{Idades de Estrelas}

A idade de uma estrela não pode ser determinada diretamente, apenas estimada, porém ela é essencial para o entendimento de como a estrela evolui, a duração de cada fase e os eventos astrofísicos pelos quais ela passa (Soderblom, 2010).

O Sol é a única estrela que possui uma idade bem acurada e precisa, mas isso se deve a medidas em laboratório de materias (meteoritos) do Sistema Solar devido ao decaimento de radionuclídeos. As características observadas no Sol não são capazes de revelar sua idade, e o método empregado para medí-la não pode ser aplicado a outras estrelas.

Geralmente a idade pode ser obtida ao se definir uma sequência de eventos e/ou fases pelas quais a estrela passa, e estimar a escala de tempo de duração de cada uma. Implicitamente a idade estimada é incerta, pois necessita da definição de um ponto inicial, ou seja, em qual momento da formação de uma estrela sua idade é igual a zero. Pontos teóricos e observacionais podem definir esse momento, tais como "linha de nascimento da queima de deutério", entrada na sequência principal de idade zero, entre outros (ver referências em Soderblom, 2010). Porém, a incerteza relacionada à definição de ponto zero, em torno de $10^{4}$ anos, não é significativa quando o interesse é obter a idade de estrelas em estágio de evolução avançado, em torno de $10^{9}$ anos.

Um indicador ideal de idade deve ser, conforme Barnes (2007), facilmente medido nas 
estrelas e não depender de muitos outros fatores; ser sensível à idade e insensível a qualquer outro parâmetro; ser calibrável usando um objeto com idade conhecida; a dependência da função deve ser invertível para fornecer a idade; seus erros devem ser mensuráveis e ao testar estrelas de mesma geração, deve-se obter resultados semelhantes. Dentre os indicadores de idade conhecidos, não há um que possua todas essas características desejáveis, logo não há um que possa ser aplicado a todos os tipos de estrelas. Geralmente eles são limitados a uma certa extensão de massa ou idade.

\subsubsection{Métodos de Estimar Idades}

Soderblom (200.9) classificou os métodos de estimativa de idades em quatro tipos, do mais confíavel ao menos: (i) fundamental, (ii) dependente de modelo, (iii) empírico e (iv) estatístico, baseado em quão bem é conhecida a física de cada um.

Idades fundamentais são aquelas em que os processos físicos envolvidos são bem conhecidos. A única idade fundamental é a estimada para o Sol, por meio de medidas de decaimento radioativo de material do Sistema Solar, e fornece uma incerteza muito pequena. Há também dois métodos que podem ser chamados de "semi-fundamentais" (Soderblom, [2010), pois necessitam apenas de algumas hipóteses que são plausíveis e não devem influenciar muito na idade determinada: (a) nucleocosmocronologia que mede o decaimento de isótopos de longa vida (urânio ou tório) de estrelas pobres em metais do disco espesso e do halo; (b) idades cinemáticas ou de expansão que consistem em traçar a órbita Galáctica de um grupo de estrelas jovens até o momento em que se encontravam próximas fisicamente, admitindo que seria o instante de sua formação.

Os métodos de estimar idades dependentes de modelos, como o nome já diz, necessitam de um modelo e hipóteses muito básicas. Esses métodos são: (a) isócronas, (b) asterosismologia e (c) limite de depleção de lítio (do inglês lithium depletion boundary).

Uma das técnicas mais utilizadas é o método de isócronas que consiste em localizar uma estrela em um modelo de isócronas (curvas de mesma idade) no diagrama HR. A física do interior estelar na sequência principal é relativamente bem conhecida, principalmente para estrelas semelhantes ao Sol, o que facilita a construção de modelos de isócronas, porém os parâmetros observacionais dessas estrelas, tais como luminosidade, temperatura efetiva e metalicidade, ainda são obtidos com incertezas que influenciam consideravelmente na 
determinação da idade. No caso de estrelas na pré-sequência principal, a física não é muito bem conhecida, gerando modelos distintos, e com isso, idades diferentes. Esse método é melhor aplicado para estrelas massivas ou mais velhas, porém ainda possui muitos pontos negativos, como uma complicada análise de seus erros, isócronas que passam pelo mesmo ponto (degenerescência) e dependência de dados observacionais precisos.

A asterosismologia estuda a estrutura interna de uma estrela através de suas diferentes frequências de pulsações. São utilizados os mesmos modelos que os da construção de isócronas, porém a asterosismologia consegue determinar mais vínculos para esses modelos (como o tamanho do envoltório ou núcleo convectivo), o que os restringem mais, fornecendo idades mais precisas $(\sim 10 \%)$. Porém, como requer observações de alta qualidade e modelos específicos para cada estrela, esse método ainda é pouco utilizado.

O método do limite de depleção de lítio é empregado para estimar a idade de aglomerados abertos jovens. O lítio é um elemento químico que é facilmente destruído em baixas temperaturas $\left(\sim 2,5 \times 10^{6} \mathrm{~K}\right)$ no interior estelar. Em estrelas de baixa massa na pré-sequência principal, o interior é completamente convectivo, o lítio da atmosfera é misturado e rapidamente destruído à medida que a temperatura do núcleo aumenta. Esse aumento de temperatura é uma função, principalmente, da massa, então a determinação da abundância de lítio restante na estrela pode ser correlacionada a sua idade. Essa técnica envolve poucas hipóteses, porém é apenas utilizada para uma pequena extensão de idades e requer espectros de boa qualidade.

As idades empíricas são estimadas pela correlação encontrada entre uma propriedade estelar observada e sua idade. Essa idade deve ser determinada a partir de outro método, tal como isócronas. As correlações são calibradas por aglomerados abertos e estrelas mais velhas com idades bem determinadas. A física dessa correlação não é muito bem entendida, contudo há explicações razoáveis para que ela exista e espera-se que a variação da idade seja monotônica. Os métodos de idades empíricas são, para estrelas de baixa massa: (a) girocronologia (do inglês gyrochronology) que relaciona o período de rotação de uma estrela com sua idade e massa, o decréscimo da rotação com a idade é bem entendido para estrelas do tipo solar; (b) atividade cromosférica ou coronal a qual decresce com a idade, sabendo-se que está relacionada com a convecção e a rotação estelar; e (c) depleção de lítio, considerando que a abundância de lítio diminui na fotosfera das estrelas com o aumento 
da idade.

As idades estatísticas são semelhantes às idades empíricas, uma vez que utilizam as correlações entre propriedades observadas das estrelas com as idades estimadas por outro método, porém tais propriedades conseguem apenas determinar uma faixa de idade, ou seja, não conseguem estimar uma idade única para cada objeto. Logo, esses métodos são válidos para fornecer a extensão de idade em que o objeto se encaixa ou a distribuição de idades de um grupo de estrelas. Há dois métodos estatísticos bem conhecidos: (a) relação idade-metalicidade: espera-se que estrelas mais velhas do disco tenham menor abundância de metais comparadas às mais novas, mas essa tendência possui grande dispersão, o que põe em dúvida a utilidade dessa relação; e (b) aquecimento do disco Galáctico: estrelas mais velhas tendem a ter movimentos espaciais maiores do que as mais jovens. Ambos os métodos serão explicados em mais detalhes nas próximas seções.

Todos esses métodos, usualmente, são utilizados para estimar idades de estrelas de massa baixa ou de aglomerados jovens. Mais detalhes podem ser obtidos em Mamajek et al. (20)(1) $)$. Ainda é difícil determinar a idade de estrelas evoluídas e/ou mais velhas, mas podemos utilizar alguns desses métodos para o estudo de estrelas centrais de nebulosas planetárias, o que será explicado na próxima seção.

\subsubsection{Métodos de Estimar Idades de ECNPS}

As ECNPs podem ter uma longa faixa de idades e não há um método desenvolvido exclusivamente para determiná-las. A necessidade em estimar a idade de ECNPs surgiu ao estudar a evolução química da Galáxia, mais especificamente a evolução temporal dos gradientes radiais de abundâncias de certos elementos químicos medidos em nebulosas planetárias, uma vez que elas fornecem abundâncias com uma boa precisão e podem ser tanto objetos jovens quanto velhos. Logo, estimar suas idades ou sua distribuição tornou-se um ponto imprescindível.

As próximas subseções explicarão alguns métodos desenvolvidos para estimar a idade desses objetos. Há métodos que só podem ser aplicados a uma amostra relativamente grande para fornecer a distribuição de idades e outros que podem produzir idades individuais. 


\subsubsection{Classificação de Peimbert}

Peimbert ([1978) propôs uma classificação para as nebulosas planetárias em quatro grupos (Tipo I, II, III e IV) baseando-se em suas abundâncias químicas de hidrogênio, hélio, carbono, nitrogênio, oxigênio e neônio, além das propriedades espaciais e cinemáticas. Esse esquema foi complementado por Peimbert e Torres-Peimbert ([198:3), Faundez-Abans e Maciel ([987) (Tipo IIa e IIb) e Maciel (1989) (Tipo V), e suas principais características são:

- Tipo I: objetos mais jovens do disco com estrelas progenitoras massivas, enriquecidos de hélio e hidrogênio, com estrutura filamentada;

- Tipo II: objetos do disco com estrelas progenitoras de massa intermediária e órbitas aproximadamente circulares;

- Tipo IIa: apresenta enriquecimento de nitrogênio entre Tipo I e Tipo IIb;

- Tipo IIb: não apresenta enriquecimento de nitrogênio.

- Tipo III: objetos do disco espesso com velocidades altas, órbitas elípticas e com distâncias maiores em relação ao plano Galáctico. Apresentam abundâncias de elementos pesados menores que as do disco fino;

- Tipo IV: objetos do halo Galáctico, com baixa abundância de elementos pesados;

- Tipo V: objetos do bojo Galáctico. Esses objetos possuem outros critérios para sua classificação (ver por exemplo Quireza et al., 2007), uma vez que possuem uma grande extensão de composição química e de massa da estrela progenitora.

A definição exata desses critérios, como os valores de abundâncias médios adotados para cada tipo, podem ser encontrados nos trabalhos mencionados anteriormente e mais recentemente na tabela 2 de Quireza et al. (20)7).

De acordo com essa classificação, os Tipos I a IV parecem reproduzir o decréscimo da massa da estrela progenitora e consequentemente, o aumento da idade da estrela central. Peimbert (1978) mencionou que NPs do Tipo I, provavelmente, corresponderiam a estrelas progenitoras com massas acima de $2,1 \mathrm{M}_{\odot}$ na $\mathrm{SP}$, e que as do Tipo II teriam estrelas progenitoras com massas em torno de $1,5 \mathrm{M}_{\odot}$. Peimbert e Serrano (匹.980) sugeriram que 
estrelas na SP com massa acima de 2,4 $\mathrm{M}_{\odot}$ gerariam NPs do Tipo I, e entre 1,0 a 2,4 $\mathrm{M}_{\odot}$, NPs do Tipo II e III. Considerando esses e outros trabalhos, Maciel (1992) estimou valores para a massa da estrela na sequência principal para cada tipo, o que está apresentado na tabela ㅁ.

A estimativa da idade da ECNP surgiu da necessidade de estudar os gradientes de abundâncias de cada tipo, para investigar a possível evidência de que eles apresentamse levemente mais acentuados na sequência de Tipos III-II-I (Maciel e Köppen, 1994). Com o intuito de comparar essa evidência com modelos numéricos de evolução química da Galáxia, esses autores adotaram a extensão de idades para cada tipo dado na tabela ㄴ.]. Esses valores decorreram de estudos que indicam que as estrelas centrais das nebulosas do Tipo I são objetos mais massivos, portanto mais jovens, e que as do Tipo II possuem abundâncias semelhantes às do Sol, logo teriam idades em torno de 5 Gano. Mais recentemente, Stasińska (2004) resumiu as idades de cada um dos tipos de Peimbert de acordo com a terceira linha da tabela ㄸ.]. As estimativas da quarta linha da tabela ㅁ. serão explicadas na próxima subseção.

Tanto as massas quanto as idades apresentadas na tabela प] são apenas aproximações e não levam em conta cálculos e modelos mais recentes para sua determinação. Além disso, existem as incertezas e os espalhamentos das abundâncias, podendo resultar em nebulosas com mais de uma classificação. Porém, podemos ter uma estimativa inicial bastante razoável das massas das estrelas progenitoras e as idades das estrelas centrais, o que é um resultado importante na busca desses valores.

Tabela 1.1 - Classificação de Peimbert relacionada à massa da estrela progenitora da NP na SP e à estimativa da idade da ECNP.

\begin{tabular}{ccccll}
\hline \hline & I & II & III & IV & \\
\hline Massa $\left(\mathrm{M}_{\odot}\right)$ & 2,4 a 8,0 & 1,2 a 2,4 & 1,0 a 1,2 & 0,8 a 1,0 & Maciel ([1992) \\
Idade (Gano) & 0 a 2 & 4 a 6 & 8 a 10 & $>10$ & Maciel e Köppen ([994) \\
& 1 & 3 & 6 & 10 & Stasińska (2004) \\
& $\lesssim 1$ a 2 & 3 a 7 & 7 a 12 & $\gtrsim 12$ & de Freitas Pacheco (11993) \\
\hline
\end{tabular}




\subsubsection{Relação Idade-Metalicidade}

A relação idade-metalicidade sugere que a metalicidade média das estrelas do disco da Galáxia deve aumentar com o tempo, uma vez que o meio interestelar se torna mais rico em metais devido à reciclagem do material provindo da nucleossíntese estelar. A metalicidade das estrelas é geralmente dada pela abundância de ferro em relação ao Sol e pode ser medida utilizando espectroscopia ou fotometria. A idade é geralmente calculada pelo método de isócronas. A relação é obtida através do estudo de uma grande amostra de estrelas, por isso é caracterizada como um método de idades estatísticas (Soderblom, $2(1) .9)$.

Essa relação foi amplamente estudada (Twarog, 1.980; Edvardsson et al., 149:3; RochaPinto et al, 2000), entre outros) e apresenta resultados semelhantes entre os diversos trabalhos, porém possui um grande espalhamento na metalicidade, maior do que a incerteza média das abundâncias medidas.

de Freitas Pacheco (149:3) derivou a abundância média de oxigênio, enxofre e argônio, e utilizou uma relação dada pela razão oxigênio-ferro para derivar a metalicidade média de cada tipo de NP, adotando para o Tipo I a metalicidade das Híades. Através da relação idade-metalicidade obtida por Nissen (19.92), de estrelas do disco e do halo, ele estimou o intervalo de idades para cada tipo da classificação de Peimbert, como mostrado na tabela ㅁ.]. Devido ao grande espalhamento da relação idade-metalicidade, as incertezas dessas idades são em torno de 3 a 4 Gano. Os intervalos de idades estimados por Maciel e Köppen (11994) são semelhantes aos de de Freitas Pacheco (199.3).

Por não ser clara a causa do espalhamento na relação idade-metalicidade e considerando que a distância Galactocêntrica da estrela pode interferir nos resultados, Eidvardsson et al. (199:3) derivaram uma relação idade-metalicidade dependente também da distância Galactocêntrica, chamada relação idade-metalicidade-distância. Essa foi a relação utilizada por Maciel et al. (2003) para estimar a idade de ECNPs e calcular a variação temporal do gradiente radial de abundância de oxigênio. Para tanto, derivaram a metalicidade das ECNPs usando a relação entre abundância de oxigênio e metalicidade obtida dos dados da amostra de Edvardsson et al. (1993), e calcularam suas distâncias Galactocêntricas. As incertezas nas idades individuais determinadas dessa forma ainda são grandes, porém o intuito do trabalho era obter uma idade relativa. 
Maciel et al. (2010) aplicaram o método baseado na relação idade-metalicidade-distância descrito anteriormente, porém com o interesse em encontrar a distribuição de idades da mesma amostra de Maciel et al. (20)3), com 234 NPs. De acordo com esse método, o pico da distribuição encontra-se entre 3 a 6 Gano, o que sugere que a maioria dessas NPs possuem progenitoras com massas na SP próximas a do Sol. Maciel et al. (2010) também utilizaram a relação idade-metalicidade de Rocha-Pinto et al. (2000) calculada para estrelas do disco da Galáxia, com idades determinadas a partir da atividade cromosférica e metalicidades provindas de dados fotométricos. A metalicidade das NPs foi calculada da mesma forma que no método anterior, com a aplicação da relação idade-metalicidade. A distribuição de idades encontrada não possui um pico acentuado, sendo mais plana. Porém em ambas as distribuições, a maioria das ECNPs possui idades abaixo de 6 Gano. A incerteza na idade individual das ENCPs, de ambos os métodos, é difícil de ser calculada, mas depois de algumas considerações, Maciel et al. (2010) chegaram à conclusão de que a incerteza média na distribuição de idades seria em torno de 1 Gano, o que não modifica o resultado encontrado.

\subsubsection{Relação Idade-Massa (N/O)}

Durante a evolução de uma estrela de massa baixa e intermediária, ocorre um processo de mistura do material, chamado dragagem (em inglês dredge-up), que modifica a abundância de determinados elementos nas camadas mais externas, enriquecendo-as com material provindo da queima nuclear. Em estrelas mais massivas, esse processo ocorre em torno de três vezes. Por essa razão, espera-se que a abundância de hélio e a razão da abundância nitrogênio-oxigênio cresçam com o aumento da massa da estrela na SP, o qual é observado nas abundâncias das NPs. Então Cazetta e Maciel (200) derivaram uma relação entre a massa da ECNP e a razão das abundâncias de N/O medida na NP.

Como a relação idade-metalicidade forneceu incertezas grandes, Maciel et al. (20)3) derivaram um método independente para verificar o comportamento da variação temporal do gradiente radial de abundância obtido com idades estimadas de maneira diferente. Com o uso (a) da relação massa da ECNP-abundância N/O de Cazetta e Maciel (20)(0); (b) de uma relação linear entre a massa inicial-massa final derivada em Maciell (2001), que conecta a massa da estrela na SP com a massa da ECNP; e (c) da relação idade- 
massa da estrela na SP obtida pelo tempo de vida médio de modelos de evolução estelar apresentados em Bahcall e Piran (198:3), Maciel et al (201)3) obtiveram uma relação idademassa (N/O) e a aplicaram para uma amostra de NPs com abundâncias conhecidas. O resultado encontrado para a variação temporal do gradiente radial, embora com algumas sobreposições, foi similar ao anterior, mostrando que métodos diferentes de estimativa de idade não modificam o comportamento relativo do gradiente. A incerteza na relação idademassa adotada está em torno de 10\%, o que forneceu uma incerteza grande para as idades calculadas, porém o método resultou em idades relativas que puderam ser empregadas para o objetivo do trabalho.

Maciel et al (2010) aplicaram esse mesmo método, porém com duas relações idademassa da estrela na SP. No Caso A, o tempo de vida é inversamente proporcional ao quadrado da massa da estrela na SP, e no Caso B, é usada a relação de Bahcall e Piran (1483). A diferença é que o Caso B aprensenta idades menores para estrelas mais massivas do que o Caso A. O pico das distribuições de idades está em torno de 3 a 7 Gano no Caso A, e de 1 a 4 Gano no Caso B, refletindo a relação massa-idade adotada. Depois de analisar as incertezas inseridas em cada relação, eles concluíram que as incertezas médias seriam 1,7 Gano e 2,9 Gano para o Caso A e B, respectivamente. Como o objetivo era o conhecimento da distribuição de idades, as incertezas não devem influenciar no comportamento delas, quando considerada uma amostra relativamente grande.

\subsubsection{Distribuições de Massa Empíricas}

É razoável esperar que a distribuição das massas de estrelas centrais de nebulosas planetárias deva ser semelhante à de anãs brancas, uma vez que a perda de massa durante a fase de nebulosa planetária é muito menor que a massa da ECNP. Com essa afirmação, Maciel et al. (2010) derivaram a distribuição de idades de ECNPs usando a distribuição de massa empírica das WD, para comparar com as distribuições encontradas pelos outros métodos que eles desenvolveram.

Inicialmente, ajustaram uma distribuição de densidade de probabilidade Gaussiana à distribuição de massa de 1175 WD encontrada por Madej et al. (20104), e usando a relação massa inicial-massa final de Maciel (2001), derivaram a distribuição de probabilidade de massa das estrelas na SP. Utilizando os mesmos Casos A e B da relação de idade-massa 
da SP citados na subseção anterior, eles estimaram a distribuição de idades das ECNPs admitindo que a taxa de formação estelar na Galáxia permaneceu constante durante o tempo de vida Galáctico. O pico da distribuição encontrada está entre 2 a 3 Gano no Caso A e em, aproximadamente, 1 Gano no Caso B. Novamente há dependência com a relação idade-massa da SP adotada, e a conclusão é que há um rápido decréscimo da distribuição para ECNPs mais velhas.

Gesicki e Zijlstra (2007) determinaram as massas de 101 ECNPs usando um método dinâmico que leva em conta a idade da NP e a temperatura da estrela central, e encontraram sua distribuição de massas. Ajustando uma distribuição de densidade de probabilidade Gaussiana a essa distribuição empírica, Maciel et al (2010) puderam estimar a distribuição de idades das ECNPs de uma maneira mais direta que a anterior, adotando novamente os Casos A e B. O pico da distribuição de ambos os casos se deslocou para idades maiores, 4 a 6 Gano para o Caso A e 2 a 4 Gano para o Caso B.

Como a relação idade-massa do Caso B parece ser mais realista e a determinação da distribuição de idades diretamente da distribuição de massas da ECNPs parece ser mais confiável, eles concluíram que a distribuição de idades de ECNPs deve possuir um pico entre 2 a 4 Gano. A distribuição de idades determinada pela relação idade-massa (N/O) (Caso B), nesse mesmo trabalho, é a que mais está de acordo com esta distribuição empírica.

\subsubsection{Isócronas}

Existem inúmeros modelos desenvolvidos para estudar a evolução de uma estrela, porém poucos incluem a fase AGB e pós-AGB, uma vez que a física nesses estágios é mais complexa e há a necessidade de considerar a convecção e mistura dos elementos químicos, pulsos térmicos, perda de massa, entre outros processos. Exemplos desses modelos são Vassiliadis e Wood (1994) e Blöcker (11995), que calcularam trajetórias evolutivas no diagrama HR para um determinado conjunto de massas e metalicidades até o estágio de anã branca.

Idiart et al. (2007) aplicaram o tradicional método de isócronas, obtidas dos modelos de Vassiliadis e Wood (1994), para determinar a idade de ECNPs da Pequena Nuvem de Magalhães, com o intuito de estudar a evolução temporal da abundância de oxigênio, derivando uma relação idade-metalicidade. É necessário calcular a temperatura efetiva, luminosidade e metalicidade da ECNP para colocá-la no diagrama HR e determinar sua 
massa, e então utilizar uma relação idade-massa para estimar sua idade. Todas essas quantidades são calculadas envolvendo algumas aproximações, o que introduz incertezas grandes no resultado e consequentemente na idade. Embora os modelos teóricos não sejam tão confiáveis para essa classe de objeto, esse método permite a determinação de uma idade individual para cada estrela. Porém, ele fornece melhores resultados para ECNPs extragalácticas, uma vez que leva em conta as suas distâncias, e como será discutido posteriormente, as distâncias de NPs Galácticas são difíceis de determinar.

\subsection{Objetivo do Trabalho}

A classificação de Peimbert fornece uma primeira avaliação sobre as idades das ECNPs, e os outros métodos mencionados resultam em estimativas razoáveis para distribuição de idades desses objetos. Entretanto, mesmo considerando somente as distribuições de idades, e não as idades individuais, esses métodos frequentemente apresentam resultados conflitantes. Por essa razão, é interessante desenvolver métodos independentes que possam ser aplicados às ECNPs. Neste projeto, pretende-se estimar a distribuição de idades a partir de correlações entre propriedades espaciais e cinemáticas das ECNPs da Galáxia, particularmente considerando o aquecimento do disco Galáctico, o que leva a uma relação entre a idade e a dispersão de velocidades das ECNPs.

O desenvolvimento deste projeto é complementar ao trabalho de Maciel et al. (2010), porém seu diferencial está em determinar as idades não considerando as abundâncias das NPs. Os principais resultados estão apresentados no artigo Kinematic ages of the central stars of planetary nebulae de Maciel, Rodrigues e Costa (201]a), anexado no apêndice Q. Parte dos resultados também foi apresentado no Simpósio 283 da União Astronômica Internacional Planetary Nebulae - An Eye to the Future, e um resumo será publicado nos proceedings do simpósio.

A amostra selecionada e os métodos para a determinação da idade são apresentados nos

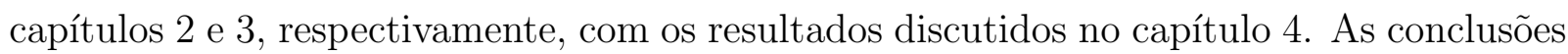
e perspectivas futuras serão discutidas no capítulo 5 . 
Capítulo 2

\section{Amostras de ECNPs}

\subsection{Introdução}

Inicialmente adotamos uma amostra de nebulosas planetárias selecionadas para estudar os gradientes de abundâncias radiais e a evolução temporal desses gradientes na Galáxia. Essa amostra foi baseada em Maciel e Köppen (19.94) e Maciel e Quireza (1199.9), com a adição de novas nebulosas, apresentadas em maior detalhe em Maciel et al. (20003, 20015, [2016). Ela também foi utilizada no primeiro trabalho de distribuições de idades das ECNPs, Maciel et al. (2010), o que facilita uma comparação direta com os métodos desenvolvidos neste trabalho. Essa amostra será chamada de Amostra A e é composta por 234 NPs com dados amplamente estudados e determinados com boa precisão na literatura. São NPs localizadas na vizinhança solar e no disco Galáctico.

Segundo os critérios de Peimbert, uma classificação preliminar da amostra pode ser feita. Para isso, utilizamos o trabalho de Quireza et al. (2007), que analisaram novamente os critérios de Peimbert em um estudo estatístico de uma amostra de 476 nebulosas. Como há objetos que possuem características de mais de um tipo e outros que não se encaixam em algum, foi aplicado o teorema de Bayes para calcular a probabilidade posterior de que uma nebulosa pertença a um dos tipos. Da Amostra A, 233 objetos foram reclassificados por esse método no trabalho de Quireza et al. (2007) e a distribuição em tipos está apresentada na tabela 2.]. Um dos objetos é classificado como pertencente a dois grupos, e cerca de $50 \%$ da Amostra A é classificada como Tipo IIa.

Como a Amostra A apresentou resultados preliminares interessantes, decidimos adotar uma segunda amostra com maior número de nebulosas planetárias para verificar se o comportamento das distribuições de idades encontradas permanecerá o mesmo, e se a 
Tabela 2.1 - Classificação de Peimbert das amostras.

\begin{tabular}{ccccccccccc}
\hline \hline \multirow{2}{*}{ Amostra } & $\#$ & I & II & IIa & IIa/IIb & IIa/III & IIb & IIb/III & III & IV \\
\hline A & 233 & 23 & 0 & 117 & 0 & 1 & 44 & 0 & 34 & 14 \\
B & 434 & 41 & 0 & 176 & 1 & 1 & 71 & 1 & 86 & 57 \\
\hline
\end{tabular}

Amostra A pode ser considerada representativa da população de NPs Galácticas. Para tanto, utilizamos o catálogo de dados cinemáticos de NPs compilados por Durand et al. (1998), visto que é necessário o conhecimento dos parâmetros cinemáticos das NPs para os métodos que desenvolvemos. Essa amostra será chamada de Amostra B e contém 866 objetos, a maioria do disco Galáctico.

Apenas 434 objetos da Amostra B foram reclassificados por Quireza et al. (2007) e a distribuição entre os tipos está na tabela [.]. Três objetos são classificados como pertencentes a dois grupos, e cerca de $40 \%$ são objetos do Tipo IIa e $20 \%$ do Tipo III.

Ambas as amostras estão apresentadas na tabela [A.] do Apêndice 田. As três primeiras colunas $(1,2,3)$ apresentam a identificação PN G (Acker et al., 1992), o nome usual e a identificação PK (Perek e Kohoutek, 1967) das NPs, respectivamente. A última coluna (9) designa qual amostra cada NP pertence. A ascensão reta e a declinação para época 1950.0 estão apresentadas na sétima e oitava colunas, respectivamente. As demais colunas (4-6) serão explicadas a seguir. Ao total são 880 objetos, e 220 NPs da Amostra A estão contidas na Amostra B. A figura [2.] apresenta a posição Galáctica de todos os objetos de ambas as amostras.

\subsection{Distâncias Heliocêntricas}

O primeiro parâmetro necessário para o desenvolvimento deste projeto é a distância heliocêntrica da ECNP. Determiná-la é muito difícil, uma vez que as NPs estão bastante afastadas para que métodos como a paralaxe trigonométrica sejam usados. Porém a distância é uma grandeza essencial para diversas investigações, como a distribuição e a taxa de nascimento de NPs, entre outras. Apenas algumas dezenas possuem distâncias derivadas com

\footnotetext{
${ }^{1} \mathrm{O}$ catálogo de Durand et al. (1998) foi publicado com 867 nebulosas planetárias, porém encontramos um erro em uma delas e decidimos retirá-la da amostra.
} 


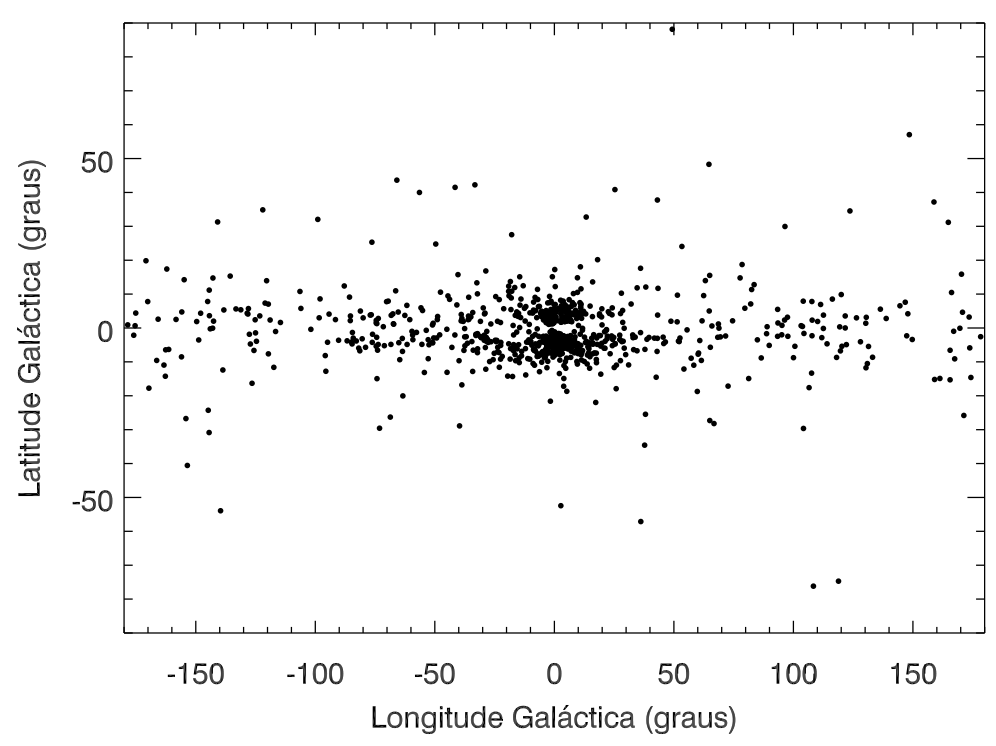

Figura 2.1: Posição Galáctica de todos os objetos das amostras.

incertezas pequenas, obtidas com métodos tradicionais, como paralaxe espectroscópica, presença de uma companheira (binária) ou ser membro de aglomerados.

A solução para obter a distância de uma amostra grande de NPs é a busca de métodos estatísticos, onde a distância (estatística) é usualmente estimada por duas ou mais propriedades da nebulosa, como fluxo, tamanho angular, densidade de elétrons, entre outras. Uma discussão sobre o problema das distâncias das NPs pode ser encontrada em Hajian (2006). A construção de uma escala de distância é feita através do desenvolvimento de um método com base na física e/ou dados empíricos, um conjunto de "calibradores" (NPs com distâncias bem conhecidas) e a análise da escala aplicada para um grupo de NPs. Diversos métodos têm sido propostos na literatura, mas ainda apresentam por vezes, distâncias contraditórias e/ou escalas de distâncias com incertezas grandes.

Um dos métodos mais conhecidos e aplicados é o de Shklovsky. Este admite que todas as NPs possuem a mesma massa ionizada e suas distâncias podem ser derivadas dos fluxos e tamanhos angulares de suas nebulosas (ver por exemplo Milne e Aller, 1975). Essa suposição é baseada no fato de que as massas das nebulosas são semelhantes, e as nebulosas costumam ser opticamente finas à radiação do contínuo de Lyman da estrela central. A densidade eletrônica pode ser escrita em termos do fluxo em 5 gigahertz $\left(F_{5 \mathrm{GHz}}\right)$, raio 
angular $(\theta)$ e distância $(d)$, e relacionada com a massa ionizada $\left(M_{i}\right)$

$$
M_{i}=7 \times 10^{-5} F_{5 \mathrm{GHz}}^{1 / 2} \varepsilon^{1 / 2} \theta^{3 / 2} d^{5 / 2}
$$

onde $\varepsilon$ é o fator de preenchimento utilizado para permitir geometria não-esférica das nebulosas; a massa é dada em $\mathrm{M}_{\odot}$, o fluxo em milijanskys, o raio angular em segundos de arco e a distância em kiloparsecs ( $(\underline{K w o k},[2000)$. Logo, a distância é dada por

$$
d=46 F_{5 \mathrm{GHz}}^{-1 / 5} \varepsilon^{-1 / 5} \theta^{-3 / 5} M_{i}^{2 / 5}
$$

Admitindo que a massa ionizada é constante $\left(M_{i} \approx 0,2 \mathrm{M}_{\odot}\right)$, a distância passa a ser dependente apenas do raio angular e do fluxo. Vários trabalhos empregaram esse método, embora sua hipótese não seja tão razoável, uma vez que a massa ionizada não é a mesma para todas as NPs, o que deve ser a principal fonte de erro para esse método.

Neste projeto serão adotadas duas escalas de distâncias heliocêntricas, com o intuito de diminuir o viés que elas possam introduzir na determinação da idade e verificar se há dependência com a escala adotada.

\subsubsection{Escala de Distância de Maciel e Pottasch}

Maciel e Pottasch (1980) determinaram a distância de NPs pelo uso de uma relação empírica entre a massa ionizada e o raio da nebulosa. Supondo um modelo esférico uniforme de nebulosa planetária com hidrogênio e hélio, a massa ionizada $\left(M_{i}\right)$ pode ser dada como

$$
M_{i}=\frac{4 \pi}{3} R_{i}^{3} \varepsilon m_{H} N_{e} \frac{1+4 y}{1+x y}
$$

onde $R_{i}$ é o raio ionizado, $\varepsilon$ é o fator de preenchimento, $m_{H}$ é a massa do hidrogênio, $N_{e}$ é a densidade numérica de elétrons, $y$ é a abundância por número de hélio, e $x=$ $N\left(\mathrm{He}^{+}\right)+2 N\left(\mathrm{He}^{++}\right) / N\left(\mathrm{He}^{+}\right) N\left(\mathrm{He}^{++}\right)$. Essa equação sugere que à medida que a massa ionizada cresce, o raio ionizado aumenta. Por isso, Maciel e Pottasch (1980) calcularam a massa e o raio ionizado (de maneira independente) para algumas nebulosas planetárias com distâncias bem determinadas, derivando assim uma relação linear empírica

$$
M_{i}\left(\mathrm{M}_{\odot}\right)=1,225 R_{i}(\text { parsec })-0,0123
$$

que deve ser válida na extensão $0,4 \gtrsim R$ (parsec) $\gtrsim 0,01$. Essa seria uma aproximação de primeira ordem para a massa ionizada da nebulosa quando comparada com a hipótese de massa constante de Shklovsky (ordem zero). 
Segundo a equação 2.2 considerando a massa ionizada constante $\left(M_{0}\right)$, a distância é $d_{0} \propto M_{0}^{2 / 5} F^{-1 / 5} \theta^{-3 / 5}$. Então a nova distância, com a aproximação de primeira ordem para a massa ionizada $(M)$, pode ser escrita como

$$
d=d_{0}\left(M / M_{0}\right)^{2 / 5}
$$

Com a relação entre o raio e a distância $R=\theta d$ e a equação [L.4, a nova distância pode ser derivada.

Maciel e Pottasch (1980) e Maciel (1981) calcularam as distâncias para 202 NPs, para as quais os fluxos são dados em Milne e Aller (1975) e Milne (11979). Posteriormente, Maciel (1984) aplicou esse mesmo método para objetos do catálogo de Cahn e Kaler (ए97]) com fluxo em $\mathrm{H} \beta$ e/ou $\mathrm{H} \alpha$ medidos, resultando em um catálogo de distâncias heliocêntricas de 468 NPs.

A incerteza de uma escala de distância é difícil de ser estimada, pois os próprios objetos calibradores são afetados por incertezas consideráveis. Maciel ([प981) estimou incertezas de $70 \%$ em média, e para os objetos mais próximos ( $d \lesssim 3 \mathrm{kpc})$, as incertezas são da ordem de $50 \%$.

Das Amostras A e B, 205 e 492 NPs, respectivamente, apresentam distâncias nesse catálogo (será referida como $d_{\mathrm{M} 84}$ ), apresentadas na quinta coluna em kpc da tabela A.] do Apêndice $\mathbb{A}$. As distribuições de distâncias heliocêntricas de ambas as amostras são apresentadas na figura [2.2. A Amostra A encontra-se concentrada até cerca de $5 \mathrm{kpc}$ do Sol, enquanto que há uma grande quantidade de objetos até 7 kpc do Sol na Amostra B.

\subsubsection{Escala de Distância de Stanghellini, Shaw e Villaver}

A escala de distância de Maciel e Pottasch (1980) é atualmente considerada como uma escala de distância "curta". Em princípio seria correto aplicá-la para objetos mais próximos ( $d \lesssim 3 \mathrm{kpc}$ ), porém seria insuficiente para objetos mais distantes, o que pode ser feito pelas chamadas "escalas longas". Uma dessas escalas é a de Stanghellini et al. (2008), os quais recalibraram o método de distâncias estatísticas desenvolvido em Cahn et al (1992), baseado em Daub (1982). A massa ionizada é escrita da seguinte forma

$$
\mu=\left(2,266 \times 10^{-21} F \theta^{3} d^{5}\right)^{1 / 2},
$$



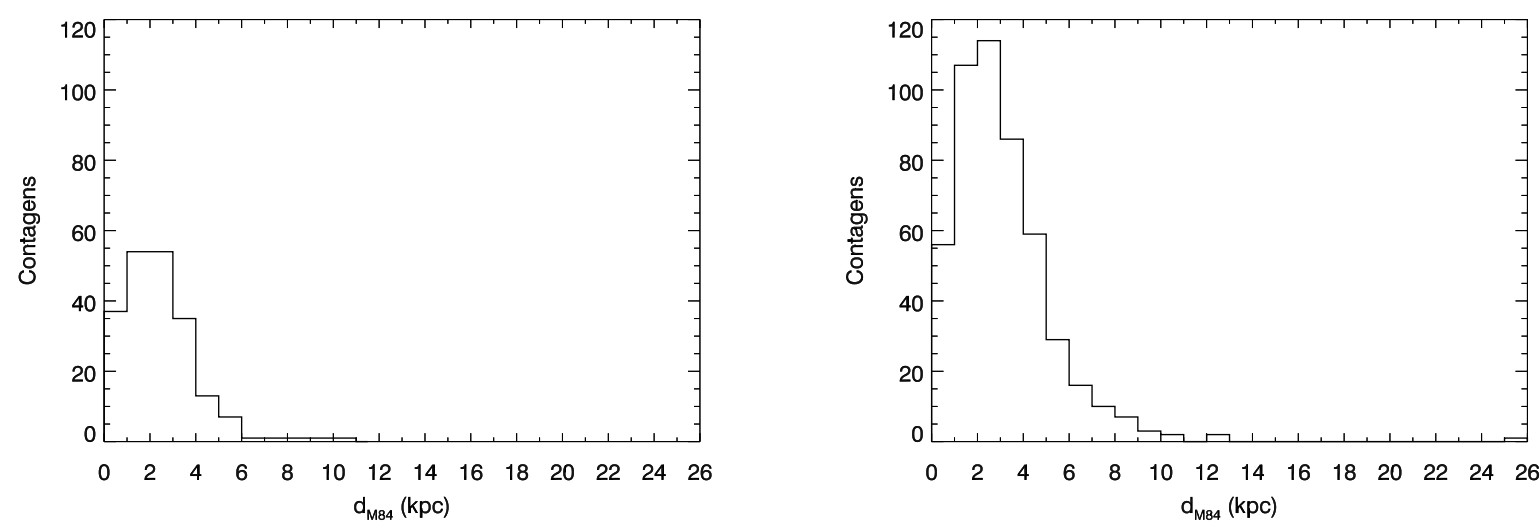

Figura 2.2: Distribuições de distâncias heliocêntricas obtidas do catálogo de Maciel (1984) da Amostra A (205 NPs), à esquerda, e B (402 NPs), à direita.

onde $F$ é o fluxo em 5 gigahertz dado em janskys, $\theta$ é o raio angular em segundos de arco, e a distância $d$ é dada em parsecs. O valor de $\log \mu$ é relacionado ao parâmetro de espessura óptica

$$
\tau=\log \frac{4 \theta^{2}}{F} .
$$

A massa ionizada $(\mu)$ aumenta enquanto a nebulosa se expande, e o grau de sua espessura óptica diminui.

Cahn et all (1992) calcularam $\mu$ e $\tau$ para 19 NPs com distâncias conhecidas e fluxos obtidos, e encontraram a seguinte relação

$$
\log \mu=\left\{\begin{array}{lc}
\tau-4, & \tau<3,13 \\
-0,87, & \tau>3,13,
\end{array}\right.
$$

sendo que em $\tau \sim 3,13$ é a transição da função de nebulosas opticamente espessas para opticamente finas.

Pelas observações dos parâmetros das NPs das Nuvens de Magalhães pelo Hubble Space Telescope, Stanghellini et al. (2008) recalibraram essa escala de distância. Adotaram a distância das Nuvens de Magalhães como a distância das NPs, pois a variação das distâncias individuais dentro das Nuvens é muito pequena. Com uma maior quantidade de calibradores (70 NPs de ambas as Nuvens de Magalhães), derivaram uma nova relação

$$
\log \mu= \begin{cases}1,21 \tau-3,39, & \tau<2,1 \\ -0,86, & \tau>2,1,\end{cases}
$$


onde a sequência de opticamente espessa é melhor definida (apresenta mais NPs) do que na relação de Cahn et al. (1992), e a sequência de opticamente fina é semelhante, porém aumenta levemente a massa ionizada, o que torna as distâncias das NPs um pouco maiores. Essa nova escala de distância foi aplicada ao catálogo de Cahn et al. (11992), e as distâncias de cerca de 560 NPs foram revisadas.

A escala de Stanghellini et al. (2018) é a mais recente publicada, e a utilização de objetos das Nuvens de Magalhães como calibradores assegura incertezas relativamente baixas. Os autores sugerem valores da ordem de 30\%, o que pode ser aplicado aos objetos mais próximos, com erros um pouco maiores para os objetos mais distantes.

A Amostra A apresenta 181 NPs com distâncias derivadas por esse método (será referida como $\left.d_{\mathrm{SSV} 08}\right)$, enquanto que a Amostra B apresenta 405 NPs, exibidas na sexta coluna em kpc da tabela A.] do Apêndice [A. Ambas as distribuições das distâncias heliocêntricas das amostras estão apresentadas na figura [2.3. A Amostra A está concentrada até em torno de $7 \mathrm{kpc}$ do Sol, enquanto que a B apresenta objetos até $11 \mathrm{kpc}$.
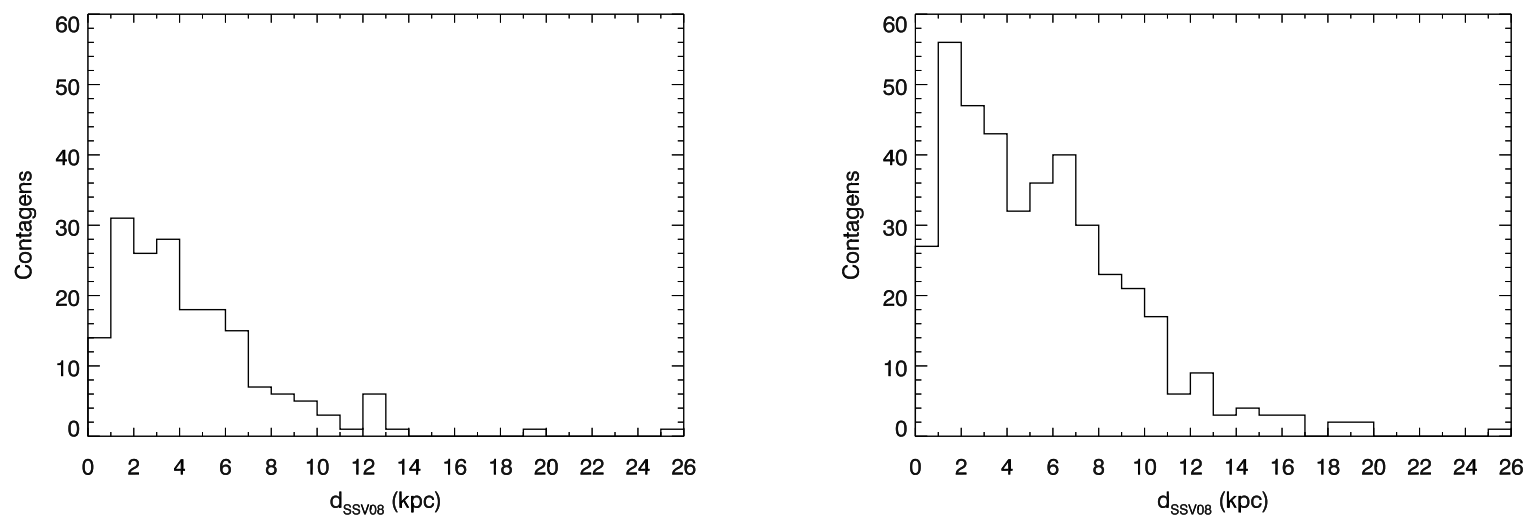

Figura 2.3: Distribuições de distâncias heliocêntricas obtidas do catálogo de Stanghellini et al. (2008) da Amostra A (181 NPs), à esquerda, e B (405 NPs), à direita.

As duas escalas de distâncias escolhidas para este projeto são obtidas por diferentes hipóteses. A escala de Maciel e Pottasch (11980) admite uma aproximação de primeira ordem para a massa ionizada das NPs em relação ao seu raio angular, enquanto que a de Stanghellini et al. (20108) considera a relação de massa ionizada de acordo com sua espessura óptica, apenas sendo constante quando a nebulosa é opticamente fina à radiação do contínuo de Lyman. A figura [2.4 apresenta a comparação dessas escalas com 363 
NPs que possuem as duas distâncias em ambas as amostras. A linha sólida representa o acordo perfeito das distâncias (relação 1:1). É claramente observado que as distâncias são muito diferentes, principalmente para objetos mais distantes $(d \gtrsim 3 \mathrm{kpc})$. A distância $d_{\mathrm{SSV} 08}$ apresenta, em geral, valores maiores que a $d_{\mathrm{M} 84}$. Elas possuem um bom acordo até aproximadamente 4 kpc. Por essa razão, é interessante utilizar as duas escalas de distância, para verificar o quanto elas podem influenciar nos resultados obtidos.

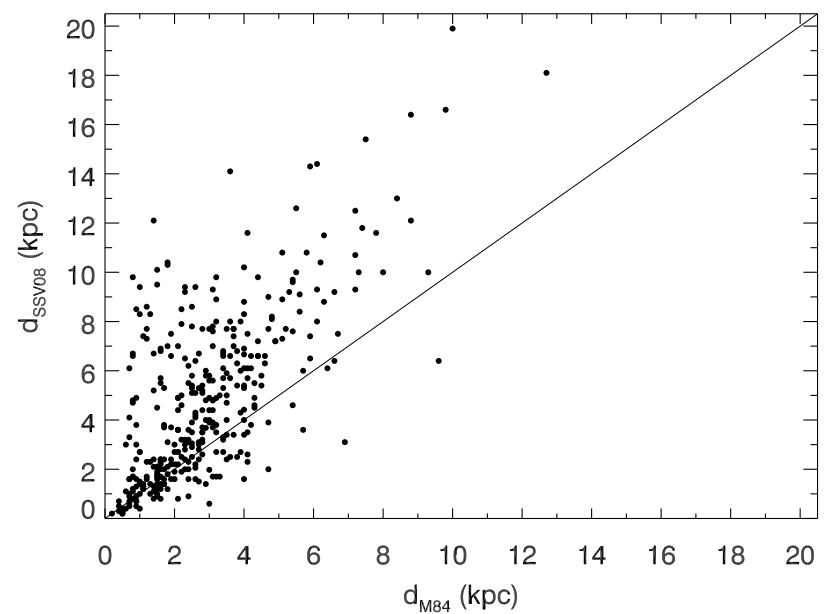

Figura 2.4: Comparação entre as distâncias obtidas pela escala de Stanghellini et al. (2008) $\left(d_{\text {SSV08 }}\right)$ com as obtidas pela escala de Maciel (1984) $\left(d_{\mathrm{M} 84}\right)$ para 363 NPs. A linha sólida representa o acordo perfeito.

\subsection{Velocidades Radiais Heliocêntricas}

O segundo parâmetro que deve ser conhecido é a velocidade radial heliocêntrica da ECNP. Durand et al. (19.98) compilaram um catálogo de dados cinemáticos de nebulosas planetárias conhecidos até aquele momento. Esse catálogo contém 867 nebulosas planetárias, sendo:

- 117 medidas de velocidades radiais de alta resolução, uma parte publicada em Zijlstra et al. (1997), 130 medidas de resolução média e 247 em baixa resolução, apresentadas no artigo;

- 373 nebulosas de Beanlien (1996), 73 de Kohoutek e Panls (1995), 52 de Dopita e Hua (1997), 3 de Spyromilio (1995) e 5 de Kraan-Korteweg et al. (11996); 
- 577 velocidades radiais publicadas no catálogo Strasbourg-ESO (Acker et al, 1992).

A média das velocidades radiais de cada nebulosa foi calculada utilizando como peso o valor inverso do erro associado ao quadrado, assim ponderando com peso menor os dados mais pobres. A maioria das nebulosas estão concentradas no disco Galáctico. Cerca de 90\% da amostra possui erros na velocidade radial abaixo de $20 \mathrm{~km} \mathrm{~s}^{-1}$ e as maiores incertezas encontram-se nas nebulosas do bojo Galáctico, não incluídas no presente projeto.

A Amostra A apresenta 220 NPs com velocidades radiais nesse catálogo. Dessas NPs, 195 apresentam distâncias no catálogo de Maciel (1984), das quais 87\% possuem erros na velocidade radial menor ou igual a $10 \mathrm{~km} \mathrm{~s}^{-1}$; e 170 apresentam distâncias no catálogo de Stanghellini et al. (2008), sendo 85\% com erros na velocidade radial menor ou igual a $10 \mathrm{~km} \mathrm{~s}^{-1}$. Como a Amostra B é baseada nesse catálogo, todos os objetos possuem velocidades radiais, sendo 492 NPs com distâncias no catálogo de Maciel (1984) e 405, no catálogo de Stanghellini et al. (20)8), das quais 70\% e 72\% possuem erros na velocidade radial menor ou igual a $10 \mathrm{~km} \mathrm{~s}^{-1}$, respectivamente. As velocidades radiais em $\mathrm{km} \mathrm{s}^{-1}$ estão apresentadas na quarta coluna da tabela [A.] do Apêndice [A. 
Capítulo 3

\section{Idades Cinemáticas}

A classificação de Peimbert e os quatro métodos discutidos na seção ए.2.2 apresentam estimativas de idades e/ou distribuições de idades plausíveis para ECNPs. Com o intuito de estimar a idade levando em conta apenas parâmetros espaciais e cinemáticos das ECNPs, independentemente das abundâncias de elementos químicos das NPs, escolhemos a relação que revela o aquecimento do disco Galáctico. A relação idade-dispersão de velocidade correlaciona apenas o movimento espacial do objeto diretamente com sua idade, sendo um método de idades estatísticas (seção [2.]), o que resultará em uma faixa de idade na qual o objeto se encaixa ou na distribuição de idades de uma amostra grande. Como adotamos duas amostras com quantidades suficientes de objetos para uma análise estatística, esperamos encontrar uma boa estimativa da distribuição de idades para as ECNPs.

O primeiro passo é a escolha da relação idade-dispersão de velocidade que será aplicada e, posteriormente, o desenvolvimento de um método para o cálculo da dispersão de velocidade das ECNPs, para então estimar a distribuição de idades. Por essa razão, as idades são chamadas de "idades cinemáticas".

\subsection{Relação Idade-Dispersão de Velocidade}

Desde antes da década de 50, os trabalhos sobre cinemática estelar apresentaram evidências de que grupos de estrelas com características espectrais semelhantes possuíam movimentos espaciais médios diferentes, ou seja, apontavam um aumento da dispersão de velocidade com o tipo espectral. Essas características foram relacionadas com a idade estelar, mostrando que grupos de estrelas com dispersões de velocidade maiores seriam 
em média mais velhas (ver por exemplo: Roman, 1950, 1952), surgindo assim uma relação entre a idade e a dispersão de velocidade espacial. A referência clássica é Wielen (1977), onde essa relação é explicada pelas flutuações locais do campo gravitacional da Galáxia, mesmo sem o conhecimento das fontes que podem estar causando essas irregularidades. Essas flutuações promovem o aquecimento cinemático das estrelas no disco e podem ser entendidas pelo estudo empírico da relação.

O termo aquecimento refere-se ao aumento dos movimentos das estrelas do disco casualmente, ou seja, a processos que introduzem energia cinética nas componentes do movimento espacial aleatoriamente com o passar do tempo (ordström, 2009). Condições iniciais como o halo e (talvez) o disco espesso, grupos moventes ou efeitos da rotação diferencial da Galáxia, não são causados devido a processos de aquecimento. Porém, diferenciá-los nas observações e relações derivadas não é algo simples.

\subsubsection{Processos de Aquecimento do Disco}

Existem diversos processos de aquecimento do disco Galáctico propostos na literatura, além de modelos que tentam explicar os dados observacionais e investigar as possíveis fontes. A relação da dispersão de velocidade com a idade é do tipo $\sigma_{v} \propto t^{\alpha}$, onde $\alpha$ assume valores entre 0,2 e 0,5 dependendo do modelo considerado, das características da fonte, entre outros. Os principais processos de aquecimento estão resumidos a seguir.

- Spitzer e Schwarzschild (195], 195:3) sugeriram que o aumento da dispersão de velocidade para estrelas mais velhas poderia ser causado pelo encontro gravitacional com nuvens moleculares gigantes, em torno de $\sim 10^{6} \mathrm{M}_{\odot}$, encontrando $\alpha=0,33$. Lacey (1.984) verificou duas fases na evolução da dispersão de velocidade, devido à influência dessas nuvens: uma fase inicial de relaxação e outra de aumento da dispersão com o tempo de maneira estável, porém ainda com a necessidade de outro mecanismo para explicar o aquecimento observado. O valor encontrado para $\alpha$ foi de 0,25 .

- Barbanis e Woltjer (11967) mostraram que estruturas espirais em larga escala poderiam ser responsáveis pelo aumento da dispersão de velocidade das estrelas. Carlberg e Sellwood (1983) e Sellwood e Carlberg (1984) simularam numericamente o aquecimento do disco causado pelos padrões espirais, e encontraram que $\alpha$ pode assumir 
valores entre 0,2 e 0,5 (Carlberg e Sellwood, 1985). Mais recentemente, De Simone et al. (20104) encontraram valores de $\alpha$ entre 0,2 a 0,76 por simulações numéricas.

- Lacey e Ostriker (1985) propuseram a presença de objetos compactos no halo Galáctico (buracos negros com massa $\sim 10^{6} \mathrm{M}_{\odot}$ ) que penetrariam no disco, perturbando as órbitas estelares, encontrando $\alpha=0,5$.

- Acreções de galáxias satélites podem ser responsáveis por uma fração substancial do aumento da dispersão de velocidade e da escala de altura das estrelas com a idade, segundo Toth e ()striker (119.92). O disco espesso da Galáxia pode ser resultado dessas fusões (Quinn et al., ए993).

- A barra Galáctica pode influenciar no espalhamento e na distribuição das velocidades das estrelas da vizinhança Solar (Fux, [2001).

Somente as nuvens moleculares gigantes não são capazes de reproduzir o aquecimento no plano do disco, porém geram um aquecimento grande na direção vertical. Mesmo com a presença de buracos negros com massa em torno de $10^{7} \mathrm{M}_{\odot}$, o aquecimento observado do disco ainda não é bem reproduzido (Hänninen e Flynn, [2012). A presença de braços espirais transientes pode levar a uma grande extensão do expoente $\alpha$ da relação idadedispersão de velocidade, o que mostra que apenas o valor do expoente não é suficiente para discriminar a fonte de aquecimento do disco (De Simone et al, 2004). Portanto, discernir o mecanismo que causa o aquecimento não é uma tarefa simples, e modelos que consideram várias fontes devem ser desenvolvidos para um estudo mais detalhado.

Todos esses modelos apresentam um contínuo aumento da relação da dispersão de velocidade com a idade, porém Quillen e Garnett (200) encontraram que o aquecimento do disco satura entre $\approx 3$ a 9 Gano, e que o disco espesso apareceria em torno de 10 Gano, onde há um aumento notável da dispersão de velocidade. Eles utilizaram a amostra de 189 estrelas anãs F e G de Edvardsson et al. (1993). Para o estudo dessa relação com um razoável grau de confiança, é necessário a obtenção de uma amostra com grande quantidade de objetos, extensa cobertura no céu, parâmetros acurados e não ser cinematicamente tendenciosa. Portanto, o mais completo conjunto de dados que pode ser utilizado para esse fim é o recente levantamento Geneva-Copenhagen, que será explicado na próxima seção. 


\subsubsection{Levantamento Geneva-Copenhagen}

Nordström et al. (2004) determinaram metalicidade, rotação, idade, cinemática e órbita Galáctica para uma amostra, não tendenciosa cinematicamente, com cerca de 14000 estrelas anãs do tipo espectral F e G da vizinhança solar - denominado levantamento GenevaCopenhagen - com objetivo de reanalisar as relações básicas do disco Galáctico, como distribuição de metalicidade de anãs $G$, relação idade-metalicidade e relação idade-velocidade. As múltiplas observações de velocidades radiais permitiram encontrar estrelas binárias da amostra. Com a adição de paralaxes acuradas do catálogo Hipparcos (ESA, 1997) e movimento próprio do catálogo Tycho-2 (Høg et al., 2000) $)$ derivaram as componentes da velocidade espacial e os parâmetros para as órbitas das estrelas. Utilizaram fotometria uvby $\beta$ de Strömgren para determinar os parâmetros astrofísicos. As calibrações para temperatura efetiva, metalicidade, distância e idade foram revisadas e melhoradas em Holmberg et al. (2007), parte II do levantamento). Os dados do catálogo de Hipparcos foram novamente reduzidos por van Leeuwen (2007), o que melhorou os valores das paralaxes. Esses novos valores foram adotados para o levantamento e apresentados em Holmberg et al. (20109, parte III).

As idades, as massas e suas incertezas foram calculadas pelo método de interpolação e técnica computacional Bayesiana de Jørgensen e Lindegren (2005). As isócronas utilizadas foram as do grupo de Padova (Girardi et aL, 2000); Salasnich et al, 2000). As componentes $U, V$ e $W$ da velocidade espacial foram derivadas, com o sistema definido com $U$ apontando em direção ao centro Galáctico, $V$ em direção a rotação e $W$ em direção ao polo norte Galáctico. Devido as correções nas calibrações e paralaxes da amostra, todos os parâmetros astrofísicos e as relações Galácticas examinadas foram revisadas, sendo apresentadas na parte III do levantamento (Holmberg et al., 2010)9).

Esse recente levantamento contém dados cinemáticos bem determinados de uma grande quantidade de estrelas, o que o torna essencial na revisão das relações Galácticas. Por isso, utilizaremos sua relação idade-dispersão de velocidade reexaminada. Holmberg et al. $(20109)$ subdividiram a amostra de estrelas com erro na idade abaixo de $25 \%$ ( $\approx 2600$ estrelas) em 30 intervalos de 88 estrelas e para cada um, calcularam a dispersão de velocidade média para as componentes da velocidade espacial e para a velocidade total. Os gráficos 
obtidos estão na figura [.]. A relação encontrada é

$$
\log \sigma_{v}=a \log t+b
$$

onde os valores de $a$ e $b$ são apresentados na tabela 3.0 para as dispersões das componentes $U, V$ e $W$ da velocidade espacial e da velocidade total $(T)$. Nessa tabela, os valores de $a$ encontrados para a primeira $\left(a_{\mathrm{GCSI}}\right)$ e segunda $\left(a_{\mathrm{GCSII}}\right)$ versão do levantamento também estão exibidos. Os valores apresentam uma leve mudança em relação ao primeiro trabalho do levantamento, mostrando que a revisão das calibrações foi importante. As relações foram calculadas excluindo os três primeiros e três últimos intervalos (bins), para evitar o viés que pode ser introduzido devido a estruturas jovens não relaxadas e estrelas do disco espesso, respectivamente. Note que o coeficiente $a$ é o correspondente ao expoente $\alpha$ discutido na seção anterior.
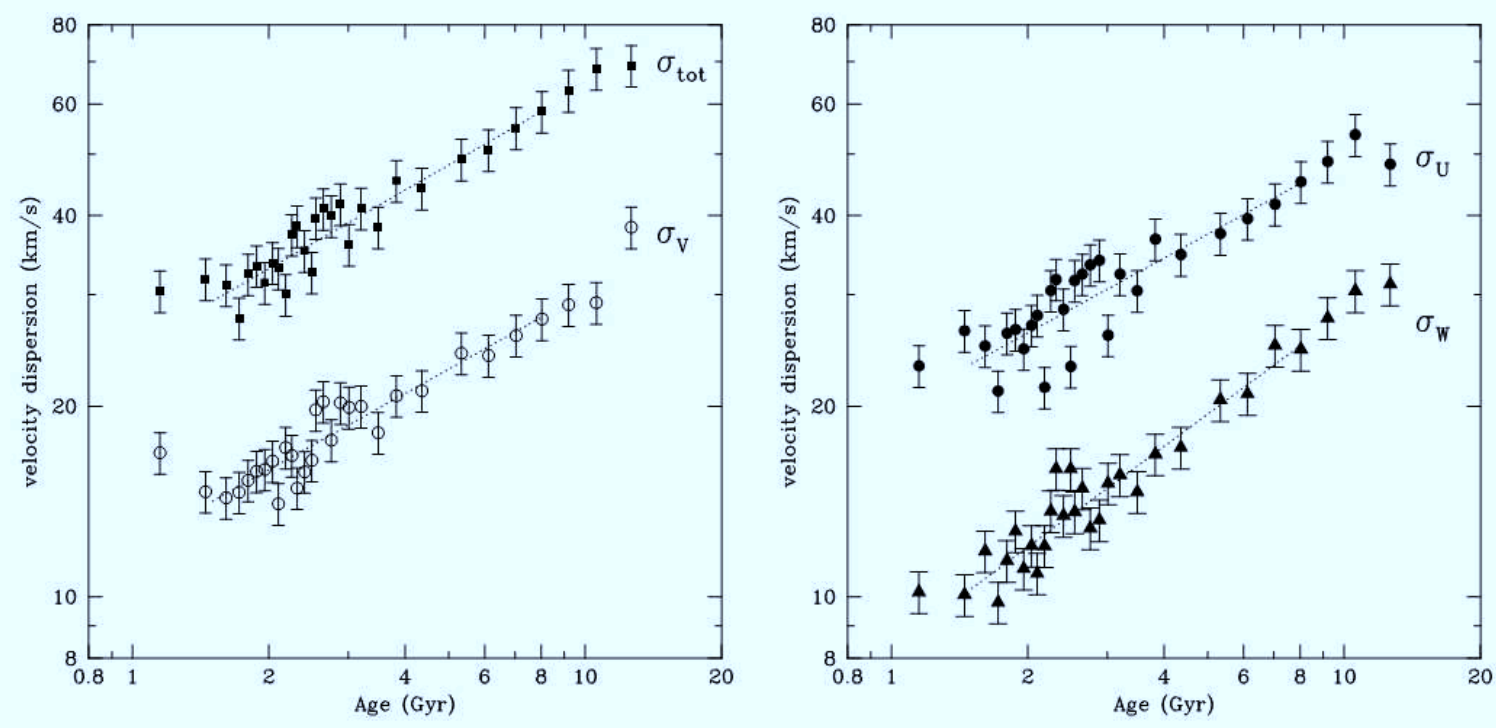

Figura 3.1: Relação idade-dispersão de velocidade das componentes de velocidade $U, V$ e $W$ e da velocidade total (tot) retirada do levantamento Geneva-Copenhagen, Holmberg et al. (200.9). A linha tracejada é o ajuste da relação excluindo os três primeiros e três últimos intervalos.

As estrelas consideradas para revisar as relações do disco Galáctico estão distribuídas em até 200 pc. Embora estejam na vizinhança solar, espera-se que as relações derivadas

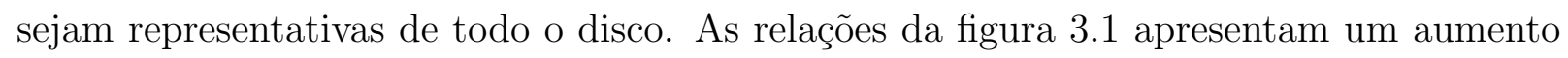
suave das dispersões de velocidade com a idade, não apresentando saturação, como encontrado por Quillen e Garnett (2001). É importante notar que esses autores utilizaram uma 
Tabela 3.1 - Coeficientes da relação idade-dispersão de velocidade.

\begin{tabular}{ccc||cc}
\hline \hline$v$ & $a$ & $b$ & $a_{\text {GCSI }}$ & $a_{\text {GCSII }}$ \\
\hline $\mathrm{U}$ & 0,39 & 1,30 & 0,31 & 0,38 \\
$\mathrm{~V}$ & 0,40 & 1,08 & 0,34 & 0,38 \\
$\mathrm{~W}$ & 0,53 & 0,92 & 0,47 & 0,54 \\
$\mathrm{~T}$ & 0,40 & 1,40 & 0,34 & 0,40 \\
\hline
\end{tabular}

amostra aproximadamente 14 vezes menor que a do levantamento Geneva-Copenhagen.

O diagrama de velocidades $\mathrm{U}-\mathrm{V}$ das estrelas do levantamento apresenta estruturas cinemáticas não aleatórias, identificadas como corrente de Hércules (Hercules stream), SiriusUMa, Coma e braço Híades-Plêiades, as quais podem interferir no reconhecimento de um mecanismo de aquecimento "puro" no disco (Nordström, 2010.9). Famaey et al. (2010.5) analisaram detalhadamente esse diagrama e utilizando uma técnica de máxima verossimilhança, identificaram distintos grupos dinâmicos. As estrelas desses grupos possuem uma ampla variação de idade e composição química, o que indica que elas não tiveram uma origem em comum. Provavelmente há algum mecanismo no disco que é capaz de aproximar essas estrelas em órbitas similares, tal como braços espirais transientes (De Simone et al, [20(14) ou a ressonância externa de Lindblad com a barra Galáctica (Fux, [2001). Além disso, as distribuições de velocidades das componentes U e V se distanciam de uma distribuição Gaussiana, indicando a presença e a influência dessas estruturas (Seabroke e (Gilmore, 20(07). Logo, a relação idade-dispersão de velocidade no plano Galáctico se torna complicada de ser obtida, e uma parametrização direta seria inadequada. A relação encontrada pelo levantamento, embora simples, provavelmente está sendo influenciada pelos movimentos não-aleatórios em pequenas escalas, o que torna difícil interpretar se o aquecimento é representativo ou se as estruturas podem estar mascarando o verdadeiro comportamento da relação.

Se alguns processos dinâmicos conduzem à formação de estruturas cinemáticas, tais como correntes (streams), onde uma população maior se reune em uma região específica do espaço de fase, essa concentração de estrelas em relação as estrelas "de fundo" pode alterar a dispersão de velocidade, por ponderá-la pela velocidade média da estrutura. 
Porém, se há de fato o aquecimento onde a contribuição dos processos dinâmicos é o aumento aleatório na direção, ocorrerá o preenchimento e a formação de um elipsóide de velocidade vagarosamente com o tempo.

Os movimentos na direção vertical $(W)$ não mostram aparentemente pequenas estruturas cinemáticas. Como a componente $\mathrm{W}$ apresenta uma distribuição aproximadamente Gaussiana em todas as idades, Seabroke e Gilmore (20107) sugeriram que o aquecimento é o termo apropriado para o aumento aleatório observado na dispersão da componente vertical. Logo, a relação descrita pela componente $W$ demonstra um aquecimento puro.

\subsection{Métodos de Estimar Idades Cinemáticas}

Soderblom (14900) calculou os movimentos espaciais para verificar a faixa de idades de algumas estrelas anãs do tipo espectral K e M, e binárias BY Draconis. Como elas são cromosfericamente ativas, espera-se que cinematicamente sejam estrelas jovens, ou seja, possuam dispersões de velocidade baixas. As dispersões de velocidades foram calculadas como em Wielen (1977). A maioria das estrelas ativas foi confirmada como cinematicamente jovem, com idades cinemáticas em torno de 0,5 Gano, e binárias BY Draconis apresentaram idades em torno de 1 a 2 Gano.

Em uma amostra de 63 anãs do tipo espectral M limitadas por volume, Reiners e Basril (200.9) calcularam seus movimentos espaciais e suas dispersões de velocidade. Eles derivaram uma idade cinemática de 3 Gano para a amostra. Outros trabalhos sobre a estimativa de idade cinemática estão citados nesse artigo.

Os dois trabalhos reportados acima destacam como o método de estimativa de idade cinemática pode ser empregado e produzir bons resultados. A relação idade-dispersão de velocidade observada no disco Galáctico pelo levantamento Geneva-Copenhagen possibilita um método de estimar idades cinemáticas, pois revela que grande quantidade de objetos do disco parece seguir a tendência de aumentar a dispersão de velocidade com o passar do tempo. Espera-se então, que as ECNPs devam seguir esse mesmo comportamento. Por isso, podemos utilizar essa relação como método para estimar a distribuição de idades das ECNPs de nossas amostras. Para tanto, precisamos calcular seus movimentos espaciais e determinar suas dispersões, o que exige o conhecimento do movimento próprio, posição espacial e velocidade radial de cada objeto. Porém, esses dados são medidos para uma 
pequena quantidade de ECNPs. Logo, há a necessidade de desenvolvermos métodos para o cálculo da dispersão da velocidade espacial, o que será explicado a seguir.

\subsubsection{Método 1: Velocidade de Rotação das NPS}

De acordo com a classificação de Peimbert, as NPs são objetos tanto jovens da População I do disco quanto de idades mais avançadas da População II do bojo ou halo. Embora com uma extensa faixa de idades, as NPs do disco descrevem uma curva de rotação bem definida e semelhante à determinada por objetos jovens, como regiões H II. Porém, apresentam uma dispersão maior, a qual é consistente com a altura média delas em relação ao plano Galáctico (Maciel e Lago, [2005), e isso pode revelar que esses objetos foram aquecidos. Logo, espera-se que a diferença entre a velocidade de rotação da NP e a velocidade de uma dada curva de rotação em sua posição seja em parte devida ao aquecimento do disco, sendo uma evidência de sua idade. Assim, neste método interpretaremos essa diferença de velocidade como a dispersão de velocidade de cada NP.

A velocidade de rotação $(\theta(R))$ de uma NP em km s${ }^{-1}$ a uma distância Galactocêntrica $R$ em kpc, admitindo órbitas circulares, pode ser escrita como

$$
\theta(R)=\frac{R}{R_{0}}\left[\frac{V_{r}}{\sin l \cos b}+\theta_{0}\right]
$$

onde $V_{r}$ é a velocidade radial relativa ao Padrão Local de Repouso dada em $\mathrm{km} \mathrm{s}^{-1}, \theta_{0} \mathrm{e}$ $R_{0}$ são a velocidade de rotação e a distância Galactocêntrica adotada para o Sol, $l$ e $b$ são a longitude e a latitude Galáctica. O Padrão Local de Repouso (LSR, sigla do inglês: Local Standard of Rest) é um ponto imaginário no plano Galáctico que se move com a velocidade média das estrelas da vizinhança solar.

Conhecida a distância heliocêntrica $(d)$ em kpc, pode-se calcular a distância galactocêntrica $(R)$ em kpc para cada NP pela relação:

$$
R^{2}=R_{0}^{2}+(d \cos b)^{2}-2 R_{0} d \cos b \cos l
$$

A velocidade radial relativa ao LSR pode ser calculada pela velocidade radial heliocêntrica $V_{r}$ (hel) usando o modelo solar padrão dado em Mihalas e Binney ([प98]) e Lang (1978), onde

$$
V_{r}=V_{r}(\text { hel })+V_{r 0} \quad \mathrm{e}
$$




$$
V_{r 0}=19,5\left(\cos \alpha_{0} \cos \delta_{0} \cos \alpha \cos \delta+\sin \alpha_{0} \cos \delta_{0} \sin \alpha \cos \delta+\sin \delta_{0} \sin \delta\right),
$$

sendo $V_{r 0}$ a velocidade radial solar relativa ao LSR, $\alpha_{0}=270,17^{\circ}$ e $\delta_{0}=29,94^{\circ}$ as coordenadas equatoriais do Sol da época 1950.0 e, $\alpha$ e $\delta$ as coordenadas equatoriais da época 1950.0 das NPs.

Então, podemos calcular a velocidade peculiar $V_{P}$ em $\mathrm{km} \mathrm{s}^{-1}$, ou seja, a diferença da velocidade de rotação da nebulosa planetária em relação à velocidade da curva de rotação $\theta_{c}$ em sua posição Galáctica:

$$
V_{P}=\Delta \theta=\left|\theta(R)-\theta_{c}(R)\right|
$$

Esperamos uma correlação entre a dispersão de velocidade e a velocidade peculiar, ou seja, $V_{P} \propto \sigma$. Logo, podemos estimar as idades das ECNPs para as quais as velocidades peculiares podem ser calculadas. Os parâmetros necessários para o cálculo desse método estão apresentados no capítulo [2, exceto a curva de rotação Galáctica que será descrita na próxima subseção.

\subsubsection{Curvas de Rotação Galácticas}

A curva de rotação revela como varia a velocidade média dos objetos de acordo com sua distância do centro da Galáxia. Seu conhecimento de forma acurada é essencial para o estudo da distribuição de massa e dinâmica da Galáxia, e é medida principalmente pelas nuvens do disco. Clemens (1985) determinou a curva de rotação usando dados de nuvens de monóxido de carbono (CO) e de hidrogênio neutro (H I) para a região interna da Galáxia e dados de nuvens de $\mathrm{CO}$ e hidrogênio ionizado (H II) para a região externa. A curva de rotação determinada é da forma

$$
\theta_{c}(R)=\sum_{i=0}^{7} c_{i} R^{i}
$$

onde $\theta_{c}(R)$ está $\mathrm{km} \mathrm{s}^{-1}$ e $R$, em kpc. Os coeficientes polinomiais $c_{i}$ foram ajustados para os valores do Sol em duas posições Galactocêntricas $R_{0}=8,5 \mathrm{kpc}$ e $R_{0}=10,0 \mathrm{kpc}$, e velocidades de rotação $\theta_{0}=220 \mathrm{~km} \mathrm{~s}^{-1}$ e $\theta_{0}=250 \mathrm{~km} \mathrm{~s}^{-1}$, respectivamente. O valor da distância Galactocêntrica recomendado para o Sol de acordo com a União Astronômica Internacional, naquela época, era $R_{0}=8,5 \mathrm{kpc}$ (Kerr e Lynden-Bell, 1986). Todavia, esse valor é atualmente considerado um limite superior, levando em conta as diversas estimativas 
recentes para a obtenção desse parâmetro, sendo encontrados valores em torno de 7,9 a 8,1 kpc (Malkin, 2012). Por essa razão, nosso grupo recalculou a curva de rotação de Clemens (11985) para os valores $R_{0}=8,0 \mathrm{kpc}$ e $\theta_{0}=220 \mathrm{~km} \mathrm{~s}^{-1}$, e os coeficientes polinomiais $c_{i}$ estão apresentados na tabela 5.2 para cada intervalo de distância Galactocêntrica. Essa foi a curva de rotação adotada como a curva "teórica" $\theta_{c}(R)$ na equação $\mathbf{3 . 5}$. Note-se que a curva de rotação é considerada constante a partir de $R=13 \mathrm{kpc}$, onde $\theta_{c}=230,6 \mathrm{~km} \mathrm{~s}^{-1}$.

Tabela 3.2 - Coeficientes do polinômio da curva de rotação Galáctica de Clemens (एप85) ajustada para $R_{0}=8,0 \mathrm{kpc} \mathrm{e} \theta_{0}=220 \mathrm{~km} \mathrm{~s}^{-1}$.

\begin{tabular}{cccccc}
\hline \hline$R(\mathrm{kpc})$ & $0-0,765$ & $0,765-2,9$ & $2,9-3,825$ & $3,825-13,0$ & $>13,0$ \\
\hline$c_{0}$ & 0,0 & 325,0912 & 329,8 & $-2346,0$ & 230,6 \\
$c_{1}$ & 3069,81 & $-248,1467$ & $-250,1$ & 2507,60391 & - \\
$c_{2}$ & $-15809,8$ & 231,87099 & 231,87099 & $-1024,068760$ & - \\
$c_{3}$ & 43980,1 & $-110,73531$ & $-110,73531$ & 224,562732 & - \\
$c_{4}$ & $-68287,3$ & 25,073006 & 25,073006 & $-28,4080026$ & - \\
$c_{5}$ & 54904,0 & $-2,110625$ & $-2,110625$ & 2,0697271 & - \\
$c_{6}$ & $-17731,0$ & - & - & $-0,080508084$ & - \\
$c_{7}$ & - & - & - & 0,00129348 & - \\
\hline
\end{tabular}

Maciel e Lago (2005) determinaram uma curva de rotação Galáctica baseada na grande amostra de nebulosas planetárias do catálogo de velocidades radiais de Durand et al. (19.98). Como esses são os objetos de estudo desta dissertação, é interessante utilizar essa curva de rotação como uma curva "teórica" alternativa na equação $\mathbf{3 . 5}$, pois é resultado do comportamento médio das NPs do disco. Eles utilizaram quatro escalas de distâncias para as NPs, porém as curvas de rotação derivadas para cada uma delas não apresentaram diferenças significativas. Foi ajustado então, um polinômio de segundo grau para os dados,

$$
\theta_{c}(R)=269,2549-14,7321 R+0,7847 R^{2}
$$

onde $R$ está em kpc e $\theta_{c}$ em $\mathrm{km} \mathrm{s}^{-1}$, calculada na extensão $4<R(\mathrm{kpc})<14$. Essa curva de rotação foi derivada levando em conta a escala de distância do catálogo de Maciell (1984), a distância Galactocêntrica do Sol $R_{0}=8,5 \mathrm{kpc}$ e a velocidade de rotação do Sol $\theta_{0}=220$ $\mathrm{km} \mathrm{s}^{-1}$. 
A diferença introduzida pelo valor adotado para $R_{0}$ é menor que $1 \%$ na velocidade de rotação considerando a equação [3.7, o que é insignificante para o objetivo deste projeto. Ambas as curvas de rotação estão apresentadas na figura 3.2. A curva sólida é a de Clemens (1085) e a tracejada, de Maciel e Lago (2005). A segunda curva mostra velocidades menores que a primeira a partir de aproximadamente $5 \mathrm{kpc}$, distanciando no máximo de $20 \mathrm{~km} \mathrm{~s}^{-1}$, mas possuem um bom acordo em geral (Maciel e Lago, 2010.5).

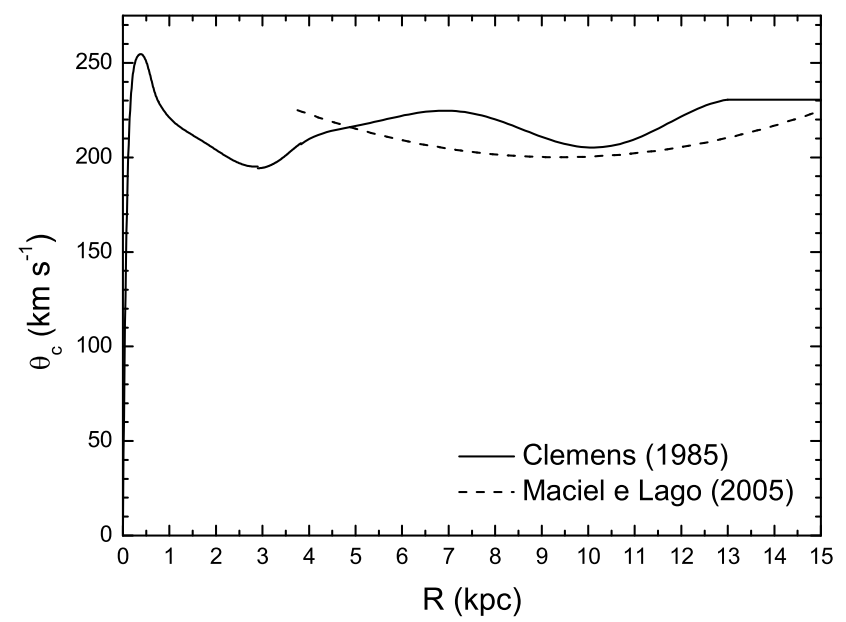

Figura 3.2: Curva de rotação Galáctica de Clemens (1985), curva sólida, e curva de rotação de Maciel e Lago (2010), curva tracejada.

\subsubsection{Método 2: Componentes U, V e W da Velocidade Espacial}

Tendo em vista as hipóteses consideradas no Método 1, desenvolvemos um segundo método mais robusto, cujas hipóteses são mais realistas. As componentes $U, V$ e $W$ da velocidade espacial podem ser calculadas conforme as seguintes relações, apresentadas em Boesgaard e Tripicco (1986):

$$
\begin{gathered}
U=-0,06715 X-0,87272 Y-0,48359 Z, \\
V=0,49274 X-0,45040 Y+0,74454 Z, \\
W=-0,86760 X-0,18837 Y+0,46020 Z,
\end{gathered}
$$

e $X, Y$ e $Z$ são dadas por

$$
X=-C \mu_{\alpha} \sin \alpha-C \mu_{\delta} \cos \alpha \sin \delta+v_{r} \cos \alpha \cos \delta+0,2,
$$




$$
\begin{gathered}
Y=C \mu_{\alpha} \cos \alpha-C \mu_{\delta} \sin \alpha \sin \delta+v_{r} \sin \alpha \cos \delta-17,2, \\
Z=C \mu_{\delta} \cos \delta+v_{r} \sin \delta+9,6 .
\end{gathered}
$$

Onde $C=4,738 / p, p$ é a paralaxe em segundos de arco, $\alpha$ e $\delta$ são a ascensão reta e a declinação para a época $1950.0, \mu_{\alpha}$ e $\mu_{\delta}$ são as componentes do movimento próprio em ascensão reta e em declinação em segundos de arco por ano, e $v_{r}$ é a velocidade radial heliocêntrica em $\mathrm{km} \mathrm{s}^{-1}$. As constantes adicionadas no final das expressões $X, Y$ e $Z$ levam em conta o movimento solar padrão, $19,7 \mathrm{~km} \mathrm{~s}^{-1}$ em direção a $\alpha=270,6^{\circ}$ e $\delta=29,2^{\circ}$. As velocidades U, V e W são definidas com U apontando em direção ao centro Galáctico, V em direção a rotação, e W em direção ao polo norte Galáctico. A velocidade espacial total $\mathrm{T}$ em $\mathrm{km} \mathrm{s}^{-1}$ é dada por

$$
T=\sqrt{U^{2}+V^{2}+W^{2}}
$$

O movimento próprio $(\mu)$ é o deslocamento angular aparente de uma estrela na esfera celeste ao longo de um ano, e é devido ao movimento verdadeiro da estrela e seu movimento relativo ao Sistema Solar. É determinado por medidas da posição da estrela, separadas por 1 ano ou mais, em relação a outros objetos considerados estacionários. Ele é decomposto em duas componentes, movimento próprio em ascensão reta $\left(\mu_{\alpha}=\mu_{\alpha}^{\prime} \cos \delta\right)$ e em declinação $\left(\mu_{\delta}\right)$ :

$$
\mu=\sqrt{\mu_{\alpha}^{\prime 2} \cos ^{2} \delta+\mu_{\delta}^{2}}=\sqrt{\mu_{\alpha}^{2}+\mu_{\delta}^{2}}
$$

onde o termo $\cos \delta$ faz a correção da escala de ascensão reta para obter a verdadeira separação angular.

A velocidade $v$ de uma estrela pode ser decomposta em uma componente ao longo da linha de visada, velocidade radial $v_{r}$, e uma componente perpendicular a linha de visada, velocidade tangencial $v_{t}$. Seu módulo é dado por

$$
v=\sqrt{v_{r}^{2}+v_{t}^{2}}
$$

Se a distância $d$ e o movimento próprio $\mu$ da estrela são conhecidos, a velocidade tangencial é encontrada por

$$
v_{t}=4,74 \mu d,
$$

onde $v_{t}$ está em $\mathrm{km} \mathrm{s}^{-1}, \mu$ em segundos de arco por ano, e $d$ em pc. 
Para calcular diretamente as componentes da velocidade espacial é necessário o conhecimento da distância (ou paralaxe), do movimento próprio e da velocidade radial do objeto para definir seu espaço de velocidade tridimensional. A distância, explicada na seção [2.2, é obtida por meio da adoção de escalas de distâncias estatísticas. A velocidade radial, explicada na seção [2.3, é um parâmetro bem conhecido, havendo catálogos próprios para NPs. Porém, o movimento próprio raramente foi obtido para esses objetos, pois estão muito distantes. Então, para o cálculo das componentes da velocidade espacial, foi necessário fazer algumas hipóteses a respeito do movimento próprio e consequentemente da velocidade tangencial das NPs:

1. Conhecendo a velocidade radial de um grande número de objetos, é razoável esperar que a média dessas velocidades deva ser aproximadamente igual à média de suas velocidades tangenciais. Portanto, admitimos que as velocidades tangenciais são aproximadamente iguais as velocidades radiais $\left(v_{t} \simeq v_{r}\right)$, o que não deve ser uma hipótese muito grosseira, pois não espera-se que haja um dado valor e direção preferenciais quando levado em conta uma grande quantidade de objetos espalhados pelo disco Galáctico.

2. As ECNPs estão muito distantes, logo seus deslocamentos angulares ao longo de um ano devem ser bastante pequenos, ou seja, seus movimentos próprios podem ser desprezados. Como primeira aproximação, negligenciamos os movimentos próprios, ou seja, consideramos $\mu_{\alpha} \simeq \mu_{\delta} \simeq 0$.

3. Em uma segunda aproximação, admitindo valores não-nulos para os movimentos próprios, esperamos que, em uma amostra significativamente grande, o movimento próprio em ascensão reta seja em média semelhante ao movimento próprio em declinação, portanto admitimos que eles, em média, são iguais $\left|\mu_{\alpha}\right| \simeq\left|\mu_{\delta}\right|$.

Os resultados obtidos com essas aproximações serão comparados, posteriormente, com os resultados da pequena amostra de NPs com determinações dos movimentos próprios.

Aplicando a hipótese do movimento próprio nulo $\mu_{\alpha} \simeq \mu_{\delta} \simeq 0$ nas expressões $X, Y$ e $Z$ em B.प, tem-se

$$
\begin{aligned}
& X=v_{r} \cos \alpha \cos \delta+0,2, \\
& Y=v_{r} \sin \alpha \cos \delta-17,2,
\end{aligned}
$$




$$
Z=v_{r} \sin \delta+9,6
$$

O termo $C$ desaparece das expressões, consequentemente $X, Y$ e $Z$ independem da paralaxe. Então, as componentes da velocidade espacial não serão dependentes da escala de distância, e sim, apenas da velocidade radial heliocêntrica. Elas foram calculadas pela equação [3.8, e a velocidade total pela equação 3.10 .

Aplicando a hipótese do movimento próprio em ascensão reta aproximadamente igual ao em declinação $\mu_{\alpha} \simeq \mu_{\delta} \simeq \bar{\mu}$ na equação do movimento próprio [.], tem-se

$$
\mu=\sqrt{\bar{\mu}^{2}+\bar{\mu}^{2}}=\sqrt{2} \bar{\mu}
$$

e substituindo na relação do movimento próprio com a velocidade tangencial em [3.13,

$$
\mu=\frac{v_{t}}{4,74 d}=\sqrt{2} \bar{\mu} \Rightarrow \bar{\mu}=\sqrt{\frac{1}{2}\left(\frac{v_{t}}{4,74 d}\right)^{2}} .
$$

Considerando a velocidade tangencial aproximadamente igual a velocidade radial, $v_{t} \simeq v_{r}$, a equação 3.16 torna-se

$$
\bar{\mu}=\sqrt{\frac{1}{2}\left(\frac{v_{r}}{4,74 d}\right)^{2}} .
$$

Portanto, podemos estimar o movimento próprio em ascensão reta e em declinação. As expressões $X, Y$ e $Z$, equação B.9, tornam-se

$$
\begin{gathered}
X=-4,738 d \bar{\mu}(\sin \alpha+\cos \alpha \sin \delta)+v_{r} \cos \alpha \cos \delta+0,2, \\
Y=4,738 d \bar{\mu}(\cos \alpha-\sin \alpha \sin \delta)+v_{r} \sin \alpha \cos \delta-17,2 \\
Z=4,738 d \bar{\mu} \cos \delta+v_{r} \sin \delta+9,6
\end{gathered}
$$

onde a paralaxe $p \sim d^{-1}$ do termo $C$ foi reescrita, $C=4,738 / p=4,738 d$. Substituindo o movimento próprio $\bar{\mu}$ pela equação [3.T7, verifica-se que o termo da distância desaparece das expressões em [3.18. Novamente elas não irão depender da escala de distância adotada, apenas da velocidade radial heliocêntrica. Logo, as componentes da velocidade espacial podem ser obtidas pela equação $\mathbf{3 . 8}$, e a velocidade total pela equação $\mathbf{3 . 1 0}$.

Para ambas as hipóteses adotadas, a dispersão de velocidade pode ser estimada da seguinte forma

$$
\sigma_{j}=\sqrt{\left(V_{j}-\overline{V_{j}}\right)^{2}}
$$

onde o índice $j$ representa as velocidades $U, V, W$ e $T$, e $\overline{V_{j}}$ representa a média aritmética das velocidades. Essa equação representa o desvio de cada ponto em relação a uma média. 
Capítulo 4

\section{Resultados e Discussão}

Derivamos as distribuições de idades cinemáticas para os dois métodos desenvolvidos no capítulo [3, aplicando os parâmetros necessários apresentados no capítulo \ para ambas as amostras. Os resultados também são discutidos em relação às aproximações do movimento próprio no método 2 e à extensão da relação idade-dispersão de velocidade adotada.

\subsection{Distribuições de Idades Cinemáticas: Método 1}

Neste método, a diferença entre a velocidade de rotação da ECNP e a velocidade prevista pela curva de rotação em sua posição Galáctica foi interpretada como a dispersão da velocidade espacial da estrela. Logo, a idade pôde ser inferida pela relação de idadedispersão de velocidade escolhida. Duas escalas de distância foram adotadas, Maciell (1984) e Stanghellini et al. (2008) (seção [2.2), para calcular a distância Galactocêntrica das ECNPs pela equação [3.3. A latitude e a longitude Galáctica foram retiradas da base de dados SIMBAD $^{\mathrm{m}}$, e adotamos como distância Galactocêntrica do Sol $R_{0}=8,0 \mathrm{kpc}$.

A figura 4.1$]$ apresenta a distribuição da distância Galactocêntrica da Amostra A, à esquerda, e da Amostra B, à direita. O histograma preto foi calculado pela escala de Maciel (1484), com 195 ECNPs da Amostra A e 492 da Amostra B; e o histograma vermelho, pela escala de Stanghellini et al. (2008), com 170 ECNPs da Amostra A e 405 da Amostra B. As ECNPs estão distribuídas entre 3 a $10 \mathrm{kpc}$ quando a distância Galactocêntrica é calculada pela escala de Maciel (1984), enquanto que há um aumento na quantidade de ECNPs nas extremidades ( $R<3 \mathrm{kpc}$ e $R>10 \mathrm{kpc}$ ), considerando a escala de Stanghellini et al. (2008).

\footnotetext{
${ }^{1}$ http://simbad.u-strasbg.fr/simbad/
} 

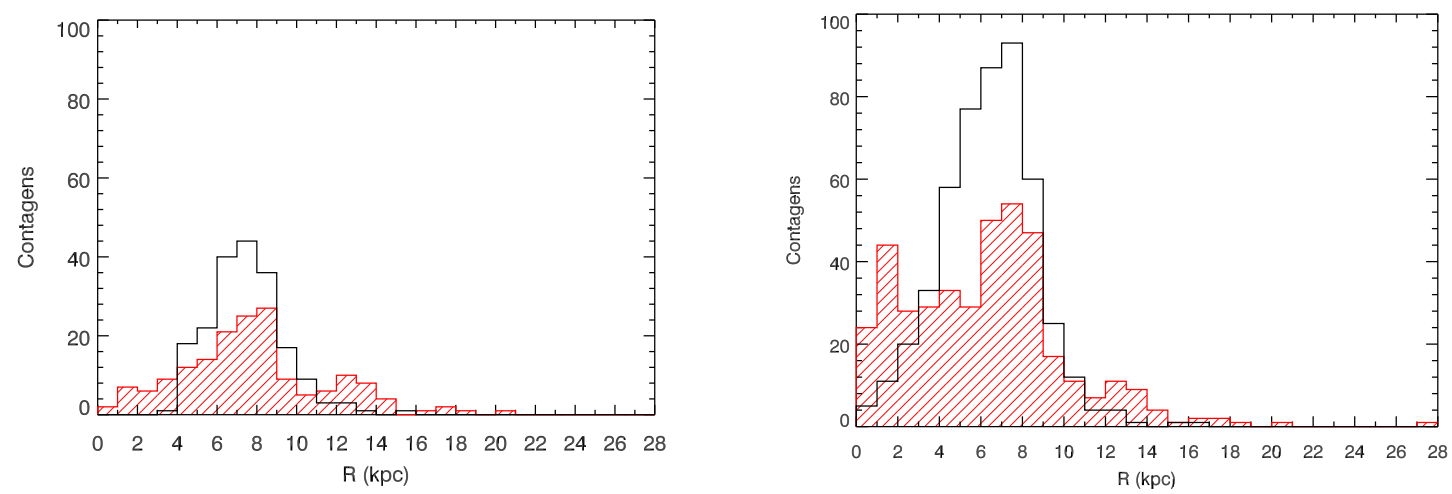

Figura 4.1: Distribuições de distâncias Galactocêntricas da Amostra A, à esquerda, e da Amostra B, à direita. O histograma preto foi calculado pela escala de Maciel (1984), com 195 ECNPs da Amostra A e 492 da Amostra B; e o histograma vermelho, pela escala de Stanghellini et al. (20018), com 170 ECNPs da Amostra A e 405 da Amostra B.

Calculamos a velocidade radial relativa ao LSR, equação [3.4, utilizando a velocidade heliocêntrica das ECNPs do catálogo de Durand et al (1998), e a ascensão reta e declinação da época de 1950.0 retirada da base de dados SIMBAD. Então, a velocidade de rotação pôde ser estimada pela equação [3.2, adotando para a velocidade de rotação do Sol $\theta_{0}=$ $220 \mathrm{~km} \mathrm{~s}^{-1}$.

Na seção [2.工工, apresentamos duas curvas de rotação da Galáxia. Uma calculada por nuvens, Clemens (1985), e outra por nebulosas planetárias, Maciel e Lago (2005). Ambas foram usadas como curvas de rotação "teóricas" quando comparadas com a velocidade de rotação da ECNP na equação [.5. De acordo com a posição Galactocêntrica da ECNP, derivamos a velocidade da curva de rotação $\theta_{c}(R)$ de Clemens (1985), pela equação [ె.6, e de Maciel e Lago (2005), pela equação 3.7. Logo, calculamos a diferença entre a velocidade de rotação da ECNP e as curvas de rotação.

Como o método 1 trata da velocidade de rotação das ECNPs, podemos considerar duas possibilidades para a velocidade peculiar: ser proporcional à (i) dispersão de velocidade da componente $V\left(\sigma_{V}\right)$ e a (ii) dispersão da velocidade total $T\left(\sigma_{T}\right)$. É intuitivo comparar com a dispersão da componente $V$ da velocidade espacial, uma vez que esta é a componente representante da rotação. Quanto à comparação com a dispersão da velocidade total, podemos admitir que as demais componentes $(U, W)$ também contribuem para o 
aquecimento.

A relação idade-dispersão de velocidade obtida pelo levantamento Geneva-Copenhagen, equação [.], é facilmente invertida para se tornar uma função da idade $t$. Portanto, utilizando os coeficientes $a$ e $b$ para a relação da dispersão da componente de velocidade $V$ e da velocidade total $T$, apresentados na tabela 3. , calculamos uma idade para cada ECNP de acordo com a dispersão encontrada. A relação foi aplicada no intervalo de $0<t($ Gano $)<14$.

Adotando as duas escalas de distâncias, as duas curvas de rotação e as duas possibilidades para a dispersão de velocidade, derivamos 8 distribuições de idades para a Amostra A e 8 para a Amostra B, denominadas $t_{1}$ a $t_{8}$ e definidas na tabela $4 . \mathbb{1}$. As distribuições de idades para cada caso bem como para a Amostra A e B estão apresentadas na figura 4.2, onde o histograma preto corresponde à Amostra $\mathrm{A}$ e o histrograma vermelho, à Amostra B. Para facilitar a comparação dos dados, os gráficos apresentam as frequências relativas de cada caso.

Tabela 4.1 - Parâmetros para o método 1.

\begin{tabular}{|c|c|c|c|c|c|}
\hline Idade & Distância & Curva de Rotação & Dispersão & $\mathrm{A}$ & B \\
\hline$t_{1}$ & Maciel (1.984) & Maciel e Lago (2005) & $\sigma_{V}$ & 74 & 149 \\
\hline$t_{2}$ & Maciel (1984) & Maciel e Lago (201).5) & $\sigma_{T}$ & 129 & 262 \\
\hline$t_{3}$ & Maciel (1.984) & Clemens (1985) & $\sigma_{V}$ & 78 & 154 \\
\hline$t_{4}$ & Maciel (1984) & Clemens (1985) & $\sigma_{T}$ & 123 & 256 \\
\hline$t_{5}$ & Stanghellini et al. (2008) & Maciel e Lago (2005) & $\sigma_{V}$ & 61 & 129 \\
\hline$t_{6}$ & Stanghellini et al. (2008) & Maciel e Lago (2005) & $\sigma_{T}$ & 99 & 209 \\
\hline$t_{7}$ & Stanghellini et al. (201)8) & Clemens (11985) & $\sigma_{V}$ & 62 & 138 \\
\hline$t_{8}$ & Stanghellini et al. (2008) & Clemens (1985) & $\sigma_{T}$ & 102 & 210 \\
\hline
\end{tabular}

Como foi admitido que as ECNPs têm órbitas circulares, aquelas que têm velocidades de rotação muito distantes da curva de rotação adotada apresentaram dispersões de velocidade grandes, o que gerou idades fisicamente não aceitáveis, ou seja, maiores que 14 Gano. Então, apenas contabilizamos nas distribuições de idades as ECNPs que possuem idades abaixo de 14 Gano, dentro do intervalo de validade da relação idade-dispersão de 

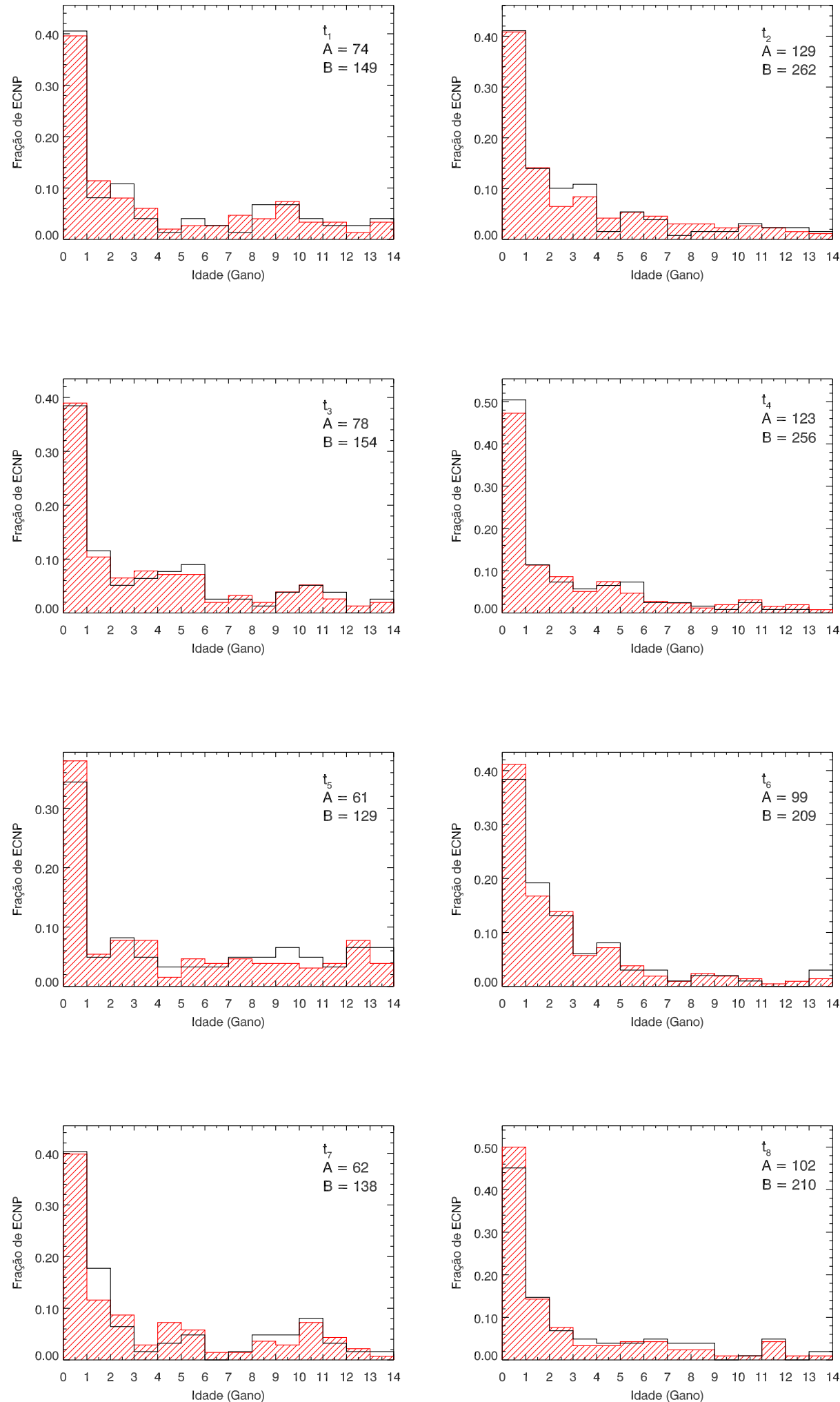

Figura 4.2: Distribuições de idades das ECNPs da Amostra A, histograma preto, e da Amostra B, histograma vermelho, pelo método 1 . 
velocidade. A quantidade de ECNPs em cada distribuição está apresentada também na tabela 4.1 para a Amostra A e B, na quinta e sexta coluna, respectivamente. Cerca de 60\% ou menos da Amostra A com dados disponíveis retornaram idades aceitáveis por esse método, enquanto que para a Amostra B, em torno de $50 \%$ ou menos.

Um comportamento geral apresentado pelas distribuições de idades do método 1 é que a maioria das ECNPs apresentam idades abaixo de 5 Gano, com uma alta concentração até 3 Gano. Para o caso onde consideramos a velocidade peculiar proporcional à dispersão da velocidade total, observa-se que há mais ECNPs nas distribuições de idades do que para o caso de $V_{P} \propto \sigma_{V}$. Isso deve-se à própria relação da idade-dispersão da velocidade total. O coeficiente angular $a(a=0,40)$ é o mesmo para ambas as relações, porém o coeficiente linear $b\left(b_{V}=1,08, b_{T}=1,40\right)$ é maior para a dispersão da velocidade total, o que é esperado, uma vez que a dispersão da velocidade total deve ser maior que a dispersão de suas componentes. Portanto, valores maiores da velocidade peculiar calculada produziram idades abaixo de 14 Gano, sendo assim contabilizadas na distribuição. Porém, examinando as frequências relativas das idades, a diferença no número de ECNPs não modifica o comportamento observado.

Ambas as amostras apresentam resultados muito semelhantes, não havendo uma distinção significativa entre elas, mesmo considerando que o número de ECNPs da Amostra B é aproximadamente o dobro da Amostra A, e que a Amostra A é mais homogênea que a Amostra B. É ainda notável que as diferenças sejam tão pequenas de um caso para outro, considerando que as escalas de distância e as curvas de rotação são diferentes.

\subsection{Distribuições de Idades Cinemáticas: Método 2}

O cálculo direto das componentes $U, V$ e $W$ da velocidade espacial requer o conhecimento da distância, velocidade radial e movimento próprio das ECNPs. A distância e a velocidade radial são parâmetros disponíveis na literatura para um grande número de NPs, porém o movimento próprio é pouco conhecido. Segundo a base de dados SIMBAD, cerca de 50 NPs de nossa amostra possuem dados sobre o movimento próprio. Esses valores variam de 0,0004 a 0,07 segundos de arco por ano, sendo muito pequenos. Portanto algumas hipóteses foram feitas para contornar a falta desse parâmetro, admitindo que a velocidade tangencial, em média, é aproximadamente igual a velocidade radial. 


\subsubsection{Movimento Próprio Nulo}

Como primeira aproximação, podemos esperar que o deslocamento angular desses objetos seja muito pequeno durante um ano e assim, negligenciamos seus valores, isto é, $\mu_{\alpha} \simeq \mu_{\delta} \simeq 0$. Isso implica em desconsiderar o primeiro e segundo termo das expressões $X$ e $Y$, e o primeiro termo da expressão $Z$, equação [3..], levando as componentes da velocidade espacial, equação [3.8, se tornarem independentes da distância do objeto, equação 3.14. Então, as componentes $U, V$ e $W$ apenas dependerão das coordenadas equatoriais e da velocidade radial das ECNPs. Por outro lado, as amostras, de acordo com a escala de distância adotada, apresentam quantidades diferentes de ECNPs. Há 25 ECNPs a mais adotando a escala de distância de Maciel (1984) do que a de Stanghellini et al. (2008), concomitantemente com dados das velocidades radiais, para a Amostra A, e 87 a mais para a mesma escala na Amostra B. É interessante notar se essa pequena diferença pode modificar algo no comportamento da distribuição de idades das ECNPs. Logo, continuaremos a dividir as amostras de acordo com a escala de distância adotada.

As velocidades radiais foram retiradas do catálogo de Durand et al] (1998), seção [2.3], e a ascensão reta e declinação para a época 1950.0 foram obtidas na base de dados SIMBAD. Calculando as expressões $X, Y$ e $Z$, obtivemos as componentes da velocidade espacial e a velocidade total $T$ pela equação 3.10 .

As distribuições das velocidades $U, V, W$ e $T$ podem ser vistas na figura 4.3 para a Amostra A, gráficos superiores, e Amostra B, gráficos inferiores. Os histogramas pretos consideram as 195 ECNPs com distâncias do catálogo de Maciel (1984), e os vermelhos, as 170 ECNPs com as distâncias do catálogo de Stanghellini et al. (201)8) para a Amostra A. Para a Amostra B os números são, respectivamente, 492 e 405 ECNPs. Os histogramas vermelhos e pretos não apresentam diferenças significativas, mostrando que a pequena variação do número de ECNPs, segundo a escala de distância adotada, não influência as distribuições de velocidades. Nota-se que as componentes da velocidade espacial se distribuem quase igualmente tanto para velocidades positivas quanto negativas, não havendo uma tendência significativa. A maioria das ECNPs possuem velocidades espaciais no intervalo de -40 a $40 \mathrm{~km} \mathrm{~s}^{-1}$, e a componente $W$ é fortemente concentrada nessa extensão, em ambas as amostras. Não há distinção significativa comparando uma amostra com a outra. 

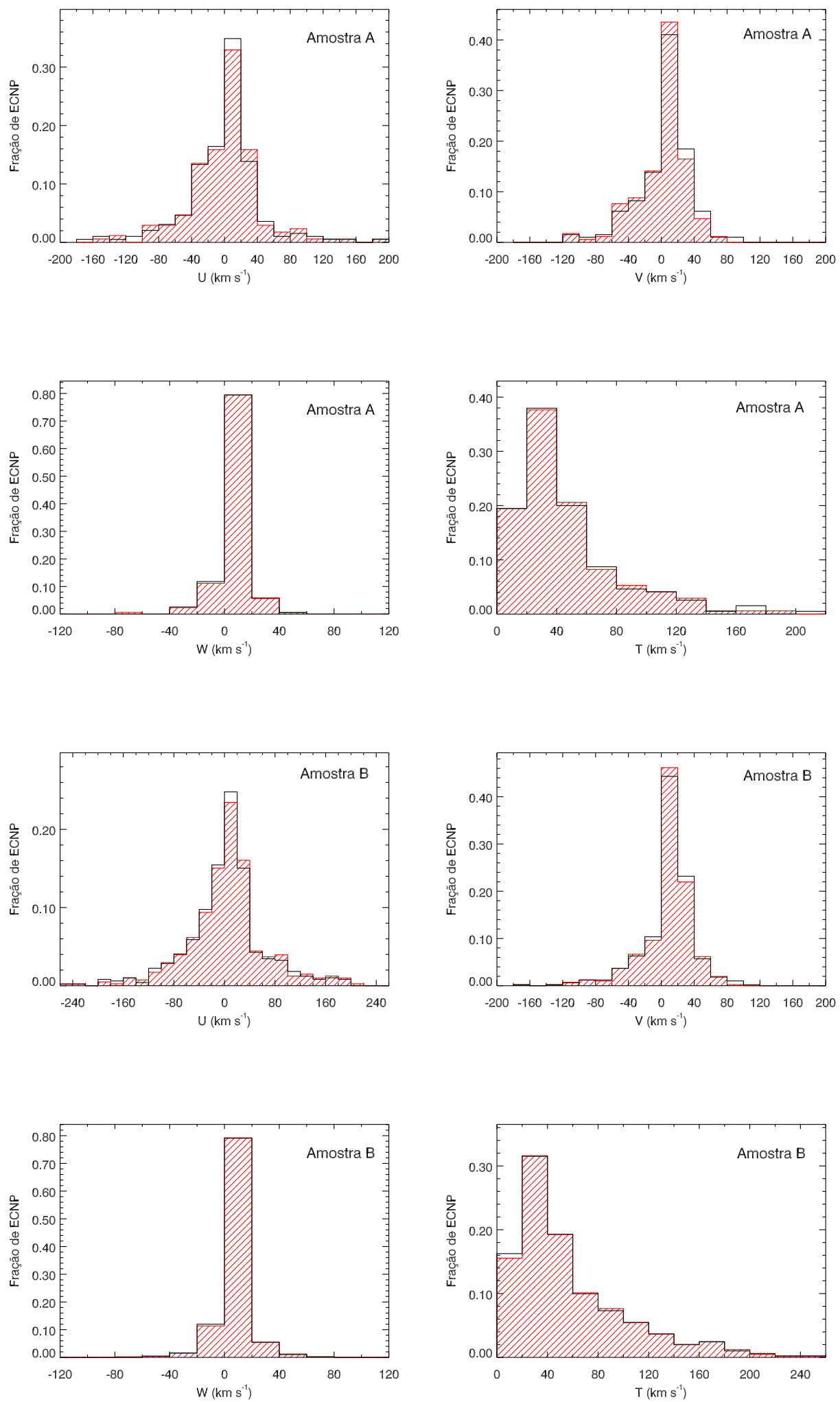

Figura 4.3: Distribuições das velocidades $U, V, W$ e $T$ para a Amostra A, gráficos superiores, e para a Amostra B, gráficos inferiores. As velocidades foram calculadas pelo método 2 com a hipótese do movimento próprio nulo. Os histogramas pretos consideram as ECNPs com distâncias do catálogo de Maciell (1984), e os vermelhos, as ECNPs com as distâncias do catálogo de Stanghellini et al. (20118). 
A média aritmética de cada componente da velocidade espacial e da velocidade total foi calculada e está apresentada em $\mathrm{km} \mathrm{s}^{-1}$ na tabela 42 para a Amostra A e B, de acordo com a escala de distância adotada. Os valores médios das componentes são pequenos e próximos a zero, como esperado segundo as distribuições de velocidades vistas na figura 4.3, e de acordo com a variação da quantidade de ECNPs em cada escala de distância, eles não modificam significativamente. As médias foram utilizadas para calcular as dispersões de velocidade de cada ECNP pela equação 3.19. Então, pela relação idade-dispersão de velocidade do levantamento Geneva-Copenhagen, equação [.], calculamos as idades referentes a cada componente e a velocidade total, utilizando os coeficientes apresentados na tabela 3.1 .

Tabela 4.2 - Média aritmética das velocidades com a hipótese do movimento próprio nulo.

\begin{tabular}{lllll}
\hline \hline & $\mathrm{A}\left(d_{\mathrm{M} 84}\right)$ & $\mathrm{A}\left(d_{\mathrm{SSV} 08}\right)$ & $\mathrm{B}\left(d_{\mathrm{M} 84}\right)$ & $\mathrm{B}\left(d_{\mathrm{SSV} 08}\right)$ \\
\hline $\bar{U}$ & $-1,33$ & 0,79 & 1,58 & 4,78 \\
$\bar{V}$ & 3,70 & 1,62 & 9,19 & 8,27 \\
$\bar{W}$ & 7,11 & 7,48 & 6,94 & 7,28 \\
$\bar{T}$ & 45,63 & 45,24 & 56,44 & 57,16 \\
\hline
\end{tabular}

Adotando ambas as escalas de distância, derivamos 8 distribuições de idades para cada amostra, denominadas de $t_{9}$ a $t_{16}$ e definidas na tabela 4.3. As distribuições de idades para cada caso e para a Amostra A e B estão apresentadas na figura 4.4, onde os histogramas pretos correspondem à Amostra A e os vermelhos, à Amostra B. Os gráficos apresentam a frequência relativa, o que facilita a comparação entre os casos e as amostras. A tabela 4.3 também mostra a quantidade de ECNPs com idades fisicamente aceitáveis, ou seja, menor que 14 Gano, e que foram contabilizadas no cálculo da frequência relativa. Algumas NPs apresentaram valores altos para as componentes da velocidade espacial, o que gera uma grande dispersão quando calculada pela maneira dada na equação $[\mathbf{L}$.9, produzindo assim idades que não devem ser reais. Comparando a quantidade de ECNPs do método 1 com o método 2, observamos que o segundo possui uma maior quantidade de objetos com idades aceitáveis, no mínimo 70\% das ECNPs com dados disponíveis em ambas as amostras. Isso indica que este método é provavelmente mais correto que o anterior. 

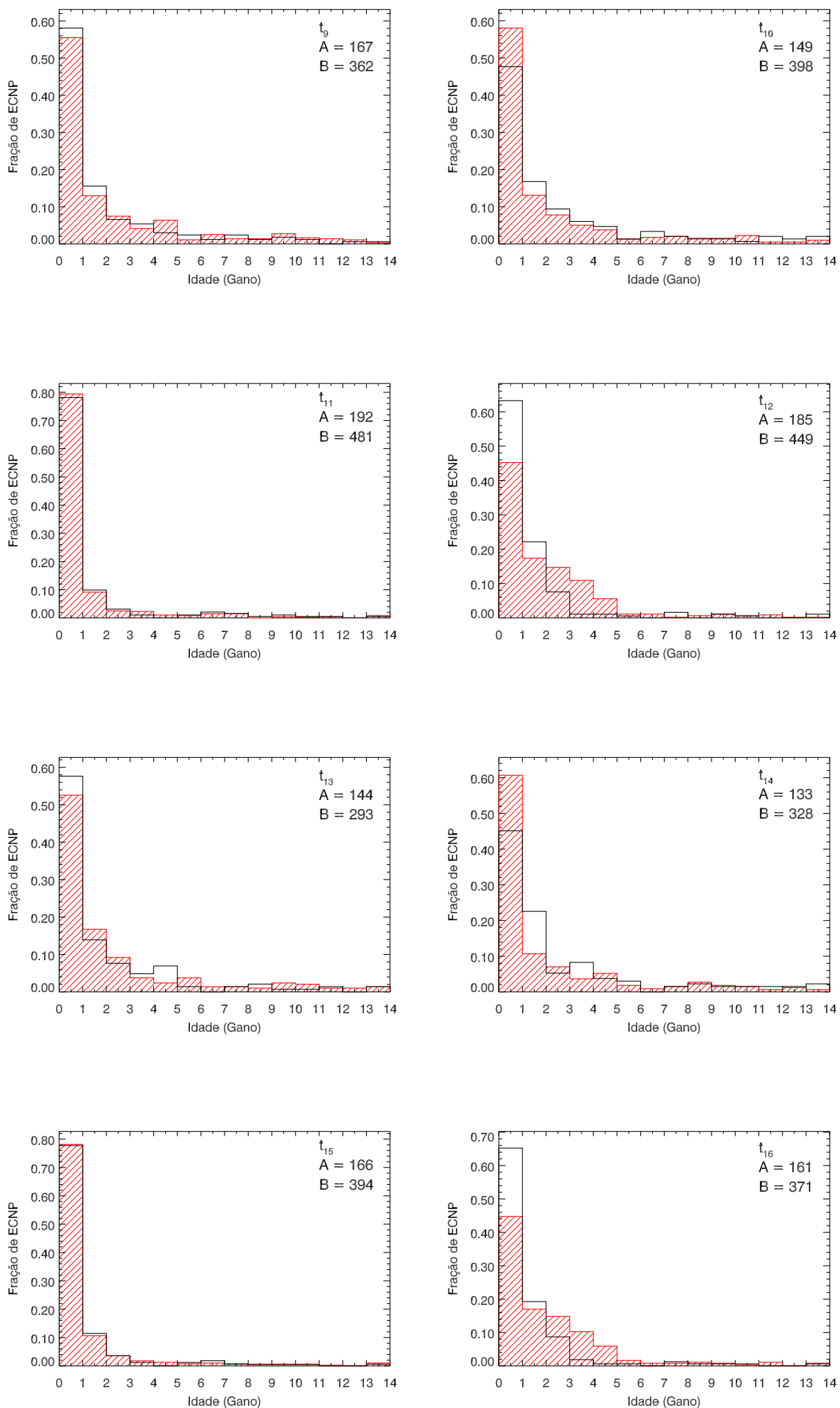

Figura 4.4: Distribuições de idades das ECNPs da Amostra A, histograma preto, e da Amostra B, histograma vermelho, pelo método 2 , admitindo a hipótese do movimento próprio nulo. 
Tabela 4.3 - Parâmetros para o método 2 com a hipótese do movimento próprio nulo.

\begin{tabular}{ccccc}
\hline \hline Idade & Distância & Dispersão & $\mathrm{A}$ & $\mathrm{B}$ \\
\hline$t_{9}$ & Maciel (11984) & $\sigma_{U}$ & 167 & 362 \\
$t_{10}$ & Maciel (11984) & $\sigma_{V}$ & 149 & 398 \\
$t_{11}$ & Maciel (11984) & $\sigma_{W}$ & 192 & 481 \\
$t_{12}$ & Maciel (11984) & $\sigma_{T}$ & 185 & 449 \\
$t_{13}$ & Stanghellini et al. (20108) & $\sigma_{U}$ & 144 & 293 \\
$t_{14}$ & Stanghellini et al. (20108) & $\sigma_{V}$ & 133 & 328 \\
$t_{15}$ & Stanghellini et al. (20108) & $\sigma_{W}$ & 166 & 394 \\
$t_{16}$ & Stanghellini et al. (201)8) & $\sigma_{T}$ & 161 & 371 \\
\hline
\end{tabular}

Novamente o comportamento geral das distribuições de idades é que a maioria das ECNPs possui idades abaixo de 5 Gano e há uma forte concentração até 3 Gano. Nota-se que a Amostra A apresenta um pico menos proeminente no primeiro intervalo de idade do que a Amostra B no histograma $t_{10}$ e $t_{14}$, referentes à dispersão da componente $V$, enquanto que a fração de ECNPs aumenta no segundo intervalo para a Amostra A. Os histogramas $t_{11}$ e $t_{15}$, referentes a dispersão de $W$, apresentam quase $80 \%$ das ECNPs no primeiro intervalo de idade, e isso é o reflexo da própria distribuição da componente $W$ da velocidade espacial, como visto na figura 4.3. Em relação à dispersão da velocidade total $T$, histogramas $t_{12}$ e $t_{16}$, a Amostra $\mathrm{A}$ distribui-se nos três primeiros intervalos de idade, enquanto que a $\mathrm{B}$, encontra-se distribuída até 5 Gano. A pequena diferença no número de ECNPs relacionado à escala de distância adotada não mostra alguma mudança no comportamento das distribuições de idades, o que pode ser visto comparando os histogramas $t_{9}$ a $t_{12}$ com os histogramas $t_{13}$ a $t_{16}$.

\subsubsection{Movimento Próprio Não-Nulo}

A segunda aproximação para o movimento próprio, admitindo que a velocidade tangencial, em média, é aproximadamente igual a velocidade radial, foi supor valores não-nulos e de mesma magnitude, ou seja, o movimento próprio em ascensão reta é aproximadamente igual ao em declinação $\left|\mu_{\alpha}\right| \simeq\left|\mu_{\delta}\right| \simeq|\bar{\mu}|$. Essa aproximação pode ser considerada como de 
primeira ordem, uma vez que não possuímos os valores do movimento próprio para a maioria das ECNPs. É razoável esperar que o deslocamento angular aparente em ascensão reta seja semelhante ao em declinação, em média, para uma amostra suficientemente grande. Porém, como visto na equação $3.17, \bar{\mu}$ pode apresentar valores tanto positivos quanto negativos, ou seja, além da suposição da magnitude, devemos considerar o sentido do movimento próprio tanto em ascensão reta quanto em declinação. Dependendo do sinal do movimento próprio, os termos das expressões $X, Y$ e $Z$ podem ser subtraídos ou somados, o que modifica seus resultados, influenciando assim as componentes da velocidade espacial. Primeiramente calculamos os valores absolutos de $\bar{\mu}$ e adotamos os valores positivos para ambos os movimentos próprios, $\mu_{\alpha} \simeq \mu_{\delta} \simeq+|\bar{\mu}|$.

Ao substituirmos a equação 3.17 do movimento próprio aproximado e o termo $C$ reescrito em função da distância $d$ nas expressões $X, Y$ e $Z$, verificamos que estas tornam-se independentes da distância novamente, equação [.]8. Como no caso anterior, há ainda a diferença na quantidade de ECNPs em cada amostra dependente da escala de distância adotada, logo continuaremos a considerar cada escala para os cálculos das componentes da velocidade espacial $U, V, W$ e a velocidade total $T$, e conferir se há mudança nas distribuições de idades determinadas.

As expressões $X, Y$ e $Z$ foram calculadas segundo a equação [3.18, adotando inicialmente valores positivos para a magnitude do movimento próprio, equação 3.17 . As componentes da velocidade espacial e a velocidade total foram calculadas pelas equações [3.8 e [3.T0, respectivamente.

As distribuições das velocidades $U, V, W$ e $T$ estão apresentadas na figura 4.5 para a Amostra A, gráficos superiores, e Amostra B, gráficos inferiores. Os histogramas pretos mostram dados de 195 ECNPs com distâncias do catálogo de Maciel (1984), e os vermelhos, 170 ECNPs com distâncias do catálogo de Stanghellini et al. (2008) para a Amostra A. Em relação a Amostra B, os valores são 492 e 405 ECNPs, respectivamente. As diferenças, devido à escala de distância adotada, são pequenas entre as amostras. No caso da velocidade $U, W$ e $T$ o pico é levemente mais proeminente para a escala de distância de Maciel (14844), sendo ao contrário para a velocidade $V$ em ambas as amostras. Há um outro intervalo de velocidade que apresenta uma concentração significativamente grande, sendo de velocidades positivas para a componente $V$, de 20 a $40 \mathrm{~km} \mathrm{~s}^{-1}$, e negativas para a compo- 
nente $U$, de 0 a $-20 \mathrm{~km} \mathrm{~s}^{-1}$. Neste caso, a velocidade $W$ apresenta uma maior extensão de velocidades, sendo a maior parte de velocidades positivas. A velocidade $V$ também apresenta a maior parte de ECNPs com velocidades positivas em ambas as amostras.

Calculamos então a média aritmética das componentes da velocidade espacial e da velocidade total, exibidas na tabela $4.4 \mathrm{em} \mathrm{km} \mathrm{s}^{-1}$, para cada amostra e escala de distância. Os valores são maiores comparados ao caso anterior e distanciam-se de zero. A velocidade $V$ apresenta médias altas e positivas, o que é observado nas distribuições da figura 4.5. A diferença de acordo com a escala de distância adotada é pequena. Conforme a equação 3.19, as médias foram utilizadas para calcular as dispersões das velocidades. Pela relação

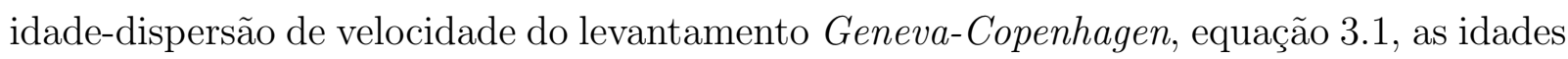
foram estimadas para cada componente da velocidade espacial e a velocidade total, equação 3.10.

Tabela 4.4 - Média aritmética das velocidades com a hipótese do movimento próprio não-nulo.

\begin{tabular}{lllll}
\hline \hline & $\mathrm{A}\left(d_{\mathrm{M} 84}\right)$ & $\mathrm{A}\left(d_{\mathrm{SSV} 08}\right)$ & $\mathrm{B}\left(d_{\mathrm{M} 84}\right)$ & $\mathrm{B}\left(d_{\mathrm{SSV} 08}\right)$ \\
\hline $\bar{U}$ & $-9,08$ & $-7,10$ & $-2,41$ & $-1,72$ \\
$\bar{V}$ & 27,57 & 23,98 & 47,40 & 46,18 \\
$\bar{W}$ & 15,32 & 18,51 & 7,68 & 8,36 \\
$\bar{T}$ & 68,83 & 68,48 & 84,21 & 84,79 \\
\hline
\end{tabular}

Como no caso anterior, adotamos as duas escalas de distância e derivamos 8 distribuições de idades para cada amostra, denominadas $t_{9}$ a $t_{16}$ e definidas na tabela 4.5 . As distribuições de idades para cada caso e amostra estão apresentadas na figura 4.6, onde os histogramas pretos correspondem à Amostra A, e os vermelhos, à Amostra B, apresentando a frequência relativa de ECNPs. A tabela 4.5 também mostra a quantidade de ECNPs que apresentam idades fisicamente aceitáveis e que foram contabilizadas nas distribuições de idades. Esses números são menores que os do caso anterior, principalmente para as velocidades $V$ e $W$, onde a extensão de velocidade obtida é ligeramente maior, ou seja, as ECNPs que apresentam velocidades muito altas, tendem a ter dispersões de velocidades grandes, gerando idades não aceitáveis por este método. Isso diminui a quantidade de ECNPs nas distribuições de idades. 

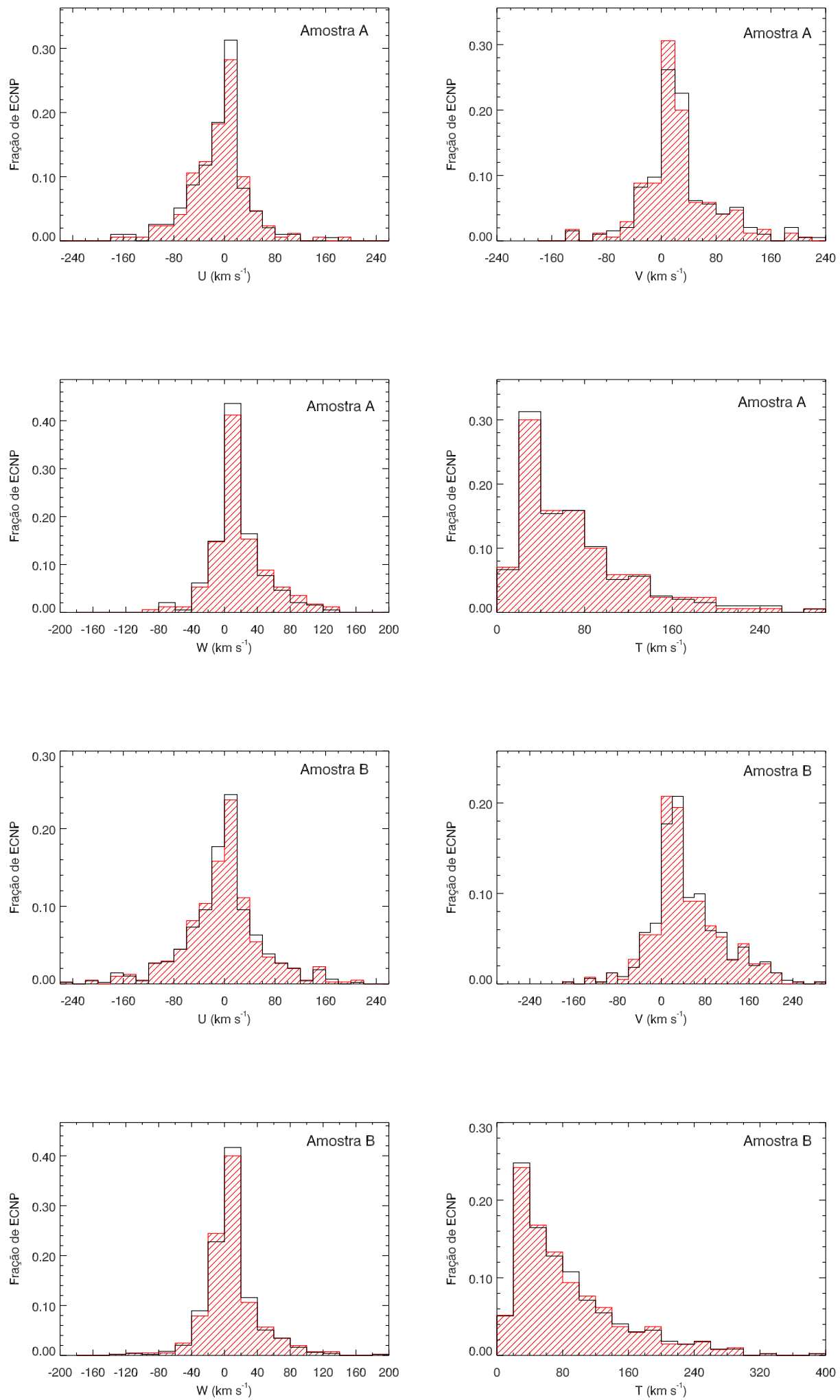

Figura 4.5: Distribuições das velocidades $U, V, W$ e $T$ para a Amostra A, gráficos superiores, e para a Amostra B, gráficos inferiores. As velocidades foram calculadas pelo método 2 com a hipótese do movimento próprio em ascensão reta aproximadamente igual ao em declinação. Os histogramas pretos consideram as ECNPs com distâncias do catálogo de Maciel (1984), e os vermelhos, as ECNPs com as distâncias do catálogo de Stanghellini et al. (2018). 
Tabela 4.5 - Parâmetros para o método 2 com a hipótese do movimento próprio não-nulo.

\begin{tabular}{ccccc}
\hline \hline Idade & Distância & Dispersão & $\mathrm{A}$ & $\mathrm{B}$ \\
\hline$t_{9}$ & Maciel (11984) & $\sigma_{U}$ & 158 & 355 \\
$t_{10}$ & Maciel (11984) & $\sigma_{V}$ & 113 & 249 \\
$t_{11}$ & Maciel (11984) & $\sigma_{W}$ & 151 & 401 \\
$t_{12}$ & Maciel (11984) & $\sigma_{T}$ & 176 & 421 \\
$t_{13}$ & Stanghellini et al. (20108) & $\sigma_{U}$ & 141 & 291 \\
$t_{14}$ & Stanghellini et al. (20108) & $\sigma_{V}$ & 102 & 207 \\
$t_{15}$ & Stanghellini et al. (20108) & $\sigma_{W}$ & 127 & 319 \\
$t_{16}$ & Stanghellini et al. (20188) & $\sigma_{T}$ & 154 & 345 \\
\hline
\end{tabular}

O comportamente geral observado nessas novas distribuições de idades é o mesmo que nas anteriores, a maioria das ECNPs possui idades menores que 5 Gano e concentrase fortemente nos primeiros intervalos de idade. Porém, apareceram algumas diferenças sutis. No caso da dispersão da componente $U$, histogramas $t_{9}$ e $t_{13}$, ambas as amostras apresentam o mesmo comportamento. Quanto à dispersão em $V$, histogramas $t_{10}$ e $t_{14}$, a Amostra A apresenta um pico bem proeminente no primeiro intervalo com cerca de $50 \%$ das ECNPs, enquanto que a Amostra B apresenta um pico inferior com aproximadamente $25 \%$ das ECNPs e o restante se espalha em idades mais avançadas. Esses são os histogramas que mais se diferenciam em relação às amostras, assim como as distribuições de velocidades deles. Os histogramas $t_{11}$ e $t_{15}$, referentes à dispersão em $W$, também se diferenciam nos dois primeiros intervalos de idades, neste caso, a Amostra B apresenta um pico mais proeminente, enquanto que a Amostra A exibe o segundo intervalo de idade com uma concentração mais próxima do primeiro intervalo, e no caso de $t_{15}$, há uma concentração significativa entre 2 a 3 Gano. As distribuições de idades da dispersão da velocidade total, histogramas $t_{12}$ e $t_{16}$, apresentam o pico mais proeminente para a Amostra $\mathrm{A}$ e um pequeno "platô" até 5 Gano, enquanto a Amostra B apresenta um suave decaimento da fração de ECNPs com a idade. A escala de distância adotada novamente não apresentou diferenças representativas. 

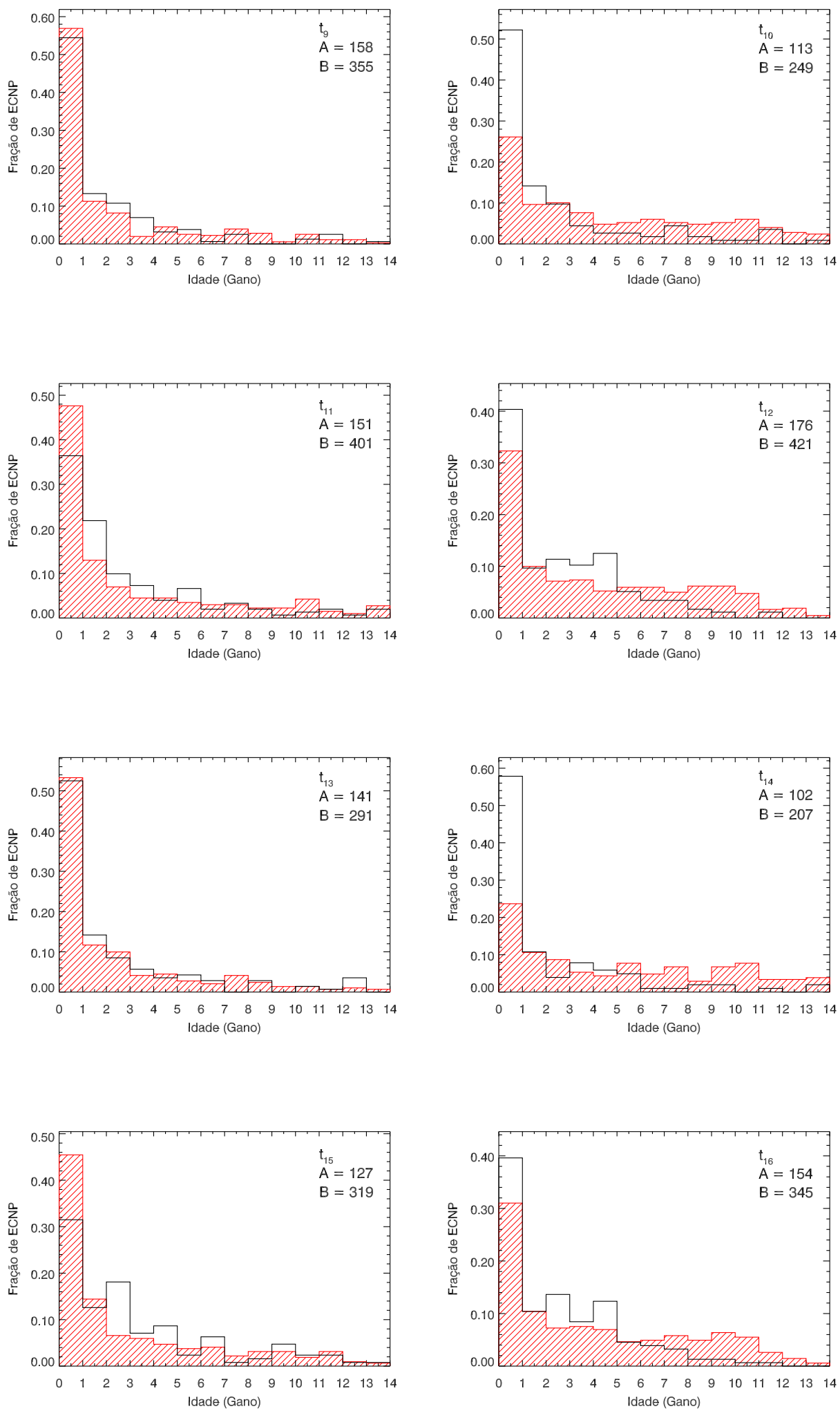

Figura 4.6: Distribuições de idades das ECNPs da Amostra A, histograma preto, e da Amostra B, histograma vermelho, pelo método 2 , admitindo a hipótese do movimento próprio em ascensão reta aproximadamente igual ao em declinação. 


\subsubsection{Teste do Sinal do Movimento Próprio}

Como era de se esperar, desprezar o movimento próprio elimina um ou dois termos das expressões $X, Y$ e $Z$, o que gera distribuições de $U, V$ e $W$ concentradas em baixas velocidades. Uma vez que admitimos um valor aproximado para aquele parâmetro, notamos uma maior extensão de velocidade das componentes, evitando um pico tão proeminente no primeiro intervalo da distribuição de idades, e as ECNPs se espalham em idades mais avançadas. Então, aproximar o valor do movimento próprio parece ser uma hipótese mais plausível e realística. Porém, há ainda a questão de seu sinal $( \pm|\bar{\mu}|)$. Os cálculos anteriores foram realizados considerando o movimento próprio positivo em ambas as coordenadas equatoriais. Seria interessante ver como as distribuições de idades variam em relação ao sinal do movimento próprio. Portanto, fizemos esse teste adotando todas as possibilidades de combinação entre os movimentos próprios: (i) $\mu_{\alpha}$ positivo e $\mu_{\delta}$ negativo, (ii) $\mu_{\alpha}$ negativo e $\mu_{\delta}$ positivo e (iii) $\mu_{\alpha}$ negativo e $\mu_{\delta}$ negativo.

Com os valores calculados do movimento próprio $\bar{\mu}$ pela equação 3.17 , alteramos apenas o sinal em $\mu_{\alpha}$ e em $\mu_{\delta}$, como nos casos citados acima, e aplicamos nas expressões $X, Y$ e $Z$, equação 3.18. Realizamos os mesmos procedimentos: calculamos as componentes $U, V$ e $W$ e a velocidade total, e derivamos as idades pelas relações idade-dispersões de velocidade do levantamento Geneva-Copenhagen. Nos dois casos anteriores, o método 2 gera 8 histogramas levando em conta a escala de distância. Como não houve alteração significativa com a escala de distância adotada, apresentaremos apenas os resultados para as ECNPs que possuem distâncias no catálogo de Maciell (1984), e utilizando apenas a Amostra B, pois possui uma maior quantidade de ECNPs. Para facilitar a comparação, uma vez que há 12 distribuições de idades novas, resolvemos apresentá-las em diagramas de extremos e quartis (em inglês, boxplot).

O boxplot é um gráfico que possibilita apresentar a distribuição de um conjunto de dados revelando apenas seus principais pontos descritivos: primeiro, segundo e terceiro quartis. Um quartil é qualquer um dos três valores que divide um conjunto de dados em 4 partes iguais. O primeiro quartil ou quartil inferior corresponde ao $25^{0}$ percentil, ou seja, é o valor ao qual se encontra $25 \%$ da amostra. O segundo quartil corresponde a mediana, ou seja, $50 \%$ da amostra. O terceiro quartil ou quartil superior corresponde a $25 \%$ da amostra com valores mais elevados da distribuição. Além disso, o boxplot pode exibir o 
$10^{\mathrm{O}}$ e o $90^{\mathrm{O}}$ percentil, ou o $5^{\mathrm{O}}$ e o $95^{\mathrm{O}}$ percentil, ou o $1^{\mathrm{O}}$ e o $99^{\mathrm{O}}$ percentil, ou o valor mínimo e máximo; como também, a média e os pontos que apresentam um grande afastamento dos demais ou são inconsistentes, chamados outliers. Logo, esse tipo de gráfico permite avaliar a simetria dos dados e sua dispersão, facilitando a comparação com outros conjuntos de dados de uma mesma grandeza.

A figura 4.7 é a representação gráfica de um boxplot. Ele tem o formato de uma caixa, onde as bordas inferior e superior representam o primeiro e terceiro quartis, respectivamente. A linha no interior representa a mediana. Neste caso, o símbolo quadrado representa a média da distribuição, e as barras inferior e superior representam o $5^{\mathrm{O}}$ e o $95^{\mathrm{O}}$ percentil.

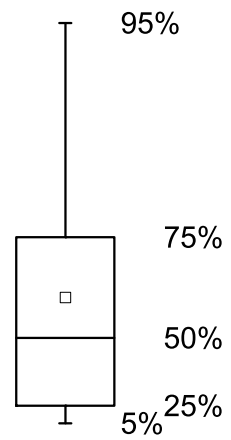

Figura 4.7: Representação gráfica de um boxplot com a descrição dos percentis.

Construímos os boxplots das idades $t_{9}, t_{10}, t_{11}$ e $t_{12}$ para cada variação do sinal do movimento próprio, incluindo a primeira hipótese de movimento próprio nulo, apresentados na figura 4.8. Para cada idade, há 5 boxplots distribuídos da seguinte maneira: movimento próprio em ascensão reta e em declinação nulos $\left(\mu_{\alpha}^{0} \mu_{\delta}^{0}\right)$, em ascensão e em declinação positivos $\left(\mu_{\alpha}^{+} \mu_{\delta}^{+}\right)$, em ascensão positivo e em declinação negativo $\left(\mu_{\alpha}^{+} \mu_{\delta}^{-}\right)$, em ascensão negativo e em declinação positivo $\left(\mu_{\alpha}^{-} \mu_{\delta}^{+}\right)$e em ascensão negativo e em declinação negativo $\left(\mu_{\alpha}^{-} \mu_{\delta}^{-}\right)$. Nestes casos, os traços inferiores e superiores representam o $5^{\mathrm{O}}$ e o $95^{\mathrm{O}}$ percentil, respectivamente. Contudo, os percentis mais importantes para o nosso objetivo são os referentes a caixa.

Todos os diagramas referentes à $t_{9}$ são muito semelhantes e bem concentrados. Apresentam $50 \%$ da amostra entre 0 e 1 Gano, e $75 \%$ possui idade abaixo de 4 Gano. No caso 

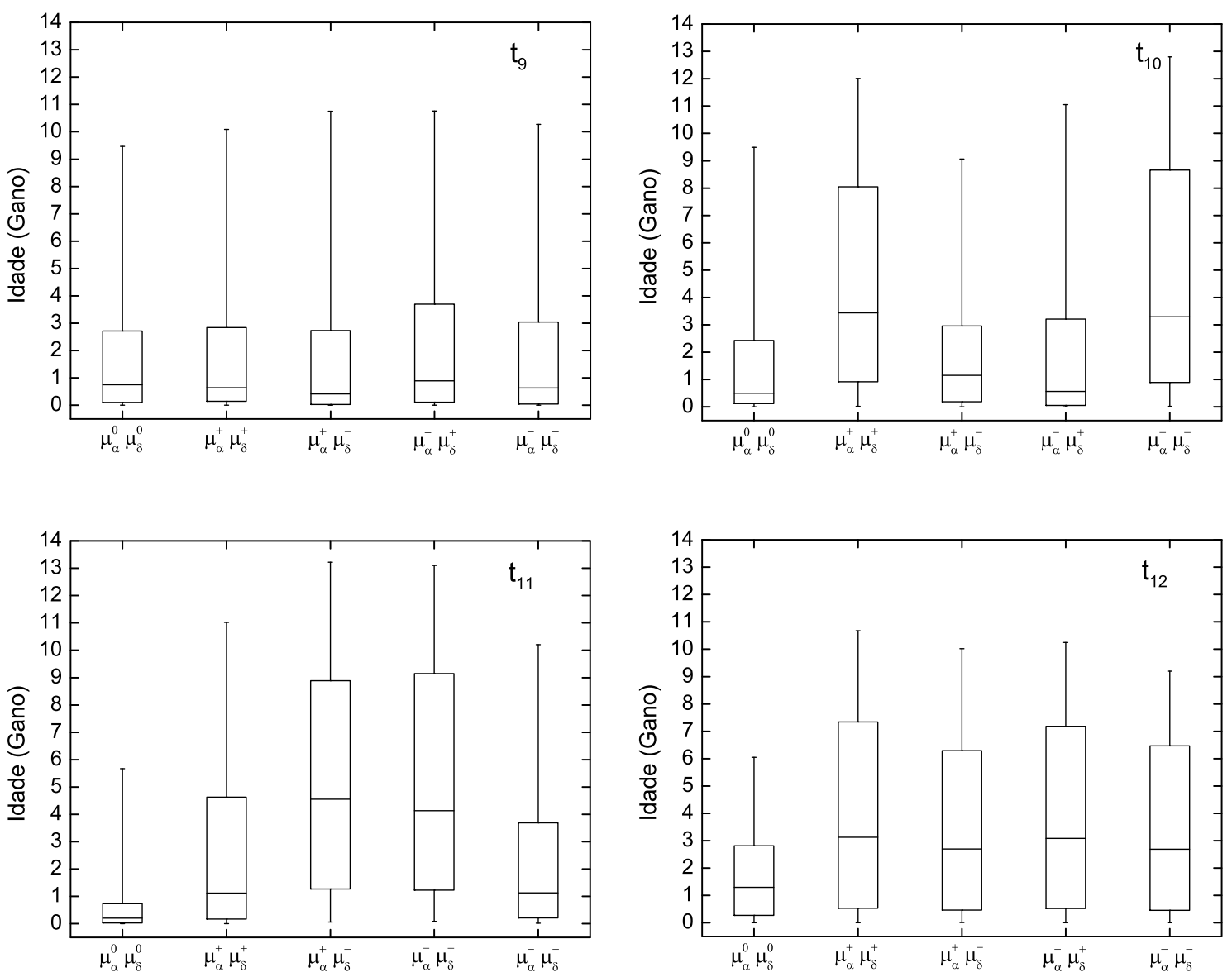

Figura 4.8: Distribuições de idades das ECNPs, apresentadas em boxplot, pelo método 2 com a Amostra B, admitindo a hipótese do movimento próprio em ascensão reta aproximadamente igual ao em declinação, e a variação de seus sinais.

de $t_{10}$, referente à dispersão na componente $V$, há uma grande variação do comportamento dependendo do sinal do movimento próprio. Na hipótese do movimento próprio nulo, o diagrama é bem concentrado e $75 \%$ da amostra apresenta idade abaixo de 3 Gano, o que já foi verificado na figura 4.4. Porém, nos casos $\mu_{\alpha}^{+} \mu_{\delta}^{+}$e $\mu_{\alpha}^{-} \mu_{\delta}^{-}$, a mediana fica entre 3 e 4 Gano e cerca de $75 \%$ da amostra possui idades distribuídas até 9 Gano, porém a maior concentração ainda está em baixas idades. O comportamento para o caso $\mu_{\alpha}^{+} \mu_{\delta}^{+}$pode ser visto em maior detalhe na figura 4.6. Nos casos $\mu_{\alpha}^{+} \mu_{\delta}^{-}$e $\mu_{\alpha}^{-} \mu_{\delta}^{+}$, os diagramas são mais concentrados e semelhantes ao caso do movimento próprio nulo, a maior parte da amostra está abaixo de 3 Gano. Para $t_{11}$, referente à componente $W$, como já notado, a concentração nos primeiros intervalos de idade é muito alta para $\mu_{\alpha}^{0} \mu_{\delta}^{0}$, e para $\mu_{\alpha}^{+} \mu_{\delta}^{+}$há uma fração sig- 
nificativa até 5 Gano, o que se repete para o diagrama de $\mu_{\alpha}^{-} \mu_{\delta}^{-}$. Nos casos $\mu_{\alpha}^{+} \mu_{\delta}^{-}$e $\mu_{\alpha}^{-} \mu_{\delta}^{+}$, os diagramas se alargam, a mediana encontra-se entre 4 e 5 Gano, e o quartil superior em torno de 9 Gano. Nota-se que esses diagramas são semelhantes aos casos $\mu_{\alpha}^{+} \mu_{\delta}^{+}$e $\mu_{\alpha}^{-} \mu_{\delta}^{-}$da idade $t_{10}$. Na idade $t_{12}$, apenas o diagrama referente ao cálculo com movimento próprio nulo é bem concentrado. Os outros diagramas são semelhantes, a mediana está em torno de 3 Gano e $75 \%$ da amostra distribui-se até 8 Gano.

O teste feito para analisar o comportamento da distribuição de idade pela mudança do sinal do movimento próprio é um pouco grosseiro. Obviamente não espera-se uma tendência no sentido do movimento próprio para as ECNPs, logo não se deve simplesmente tratar todos os objetos com sinais iguais. Um teste mais realista seria considerar sinais aleatórios para a amostra e assim verificar a distribuição de idades.

\subsubsection{Limites da Relação Idade-Dispersão de Velocidade}

Outro ponto que deve ser levado em conta é a grande quantidade de objetos no primeiro intervalo de idade, até 1 Gano. Isso ocorre devido à grande quantidade de dispersões pequenas encontradas por ambos os métodos. Todavia, a própria relação idade-dispersão de velocidade do levantamento Geneva-Copenhagen foi ajustada negligenciando os três primeiros e os três últimos pontos. Nos métodos desenvolvidos aqui, consideramos um intervalo fixo de idade $0<t($ Gano $)<14$ no qual a relação é aplicada, porém o ajuste da relação nos dados do levantamento foi feito na extensão de $1,5 \lesssim t$ (Gano) $\lesssim 8,0$ (rever figura [.]

É razóavel esperar que a dispersão continue aumentando com a idade do objeto, o que é até notado no três últimos pontos desconsiderados da relação que vão até cerca de 12,5 Gano. O motivo pelo qual foram excluídos do ajuste é a possibilidade de conterem estrelas do disco espesso. Existe também a possibilidade de que, a partir de uma certa idade, a dispersão torne-se aproximadamente constante ou varie de forma diferente. Então não há como saber ao certo como a relação idade-dispersão de velocidade se comporta no limite superior de idade. Contudo, decidimos expandir a relação até cerca de 14 Gano, como um valor limite representativo da idade da Galáxia.

No outro extremo, os três primeiros pontos foram excluídos do ajuste, pois as estrelas mais jovens podem ainda não terem tido tempo para relaxarem, e portanto, não 
apresentariam o comportamento esperado pela relação idade-dispersão de velocidade. É também notado que esses três pontos iniciais não seguem exatamente a tendência do ajuste, aparentando manter uma dispersão constante para idades mais baixas (rever figura [3.]). Seabroke e Gilmore (2007) verificaram, pela distribuição de velocidade da componente W, que as estrelas só aparentavam estar relaxadas após 2,8 Gano, quando a distribuição de W se aproximava de uma distribuição Gaussiana. Logo, a própria relação idade-dispersão de velocidade para essa componente deveria ser limitada inferiormente a essa idade. Porém, como queremos cobrir uma grande faixa de idades, pois sabemos que estrelas mais massivas evoluem em uma extensão de tempo menor, resolvemos expandir a relação até $\simeq 0$ Gano, mesmo que esses objetos provavelmente não tenham tido tempo para relaxarem. As distribuições de idades resultantes dos nossos métodos mostraram que a grande maioria das ECNPs caem nos primeiros intervalos de idades, gerando um pico muito proeminente. Portanto é importante reanalisar o primeiro intervalo e verificar como o pico da distribuição de idade varia se modificarmos o limite inferior da relação.

Para tanto, escolhemos três limites inferiores: 0,3, 0,5 e 0,7 Gano, mesmo sendo muito abaixo das idades esperadas para que os objetos estejam relaxados. Então, simplesmente excluímos as ECNPs que tivessem idades abaixo desses valores e construímos (i) histogramas de contagens, para verificar como diminui a quantidade de ECNPs no primeiro intervalo, e (ii) histogramas de frequência relativa, para notar como a distribuição de idades da amostra se rearranja após a retirada desses objetos. Aplicamos esse corte do limite inferior apenas para as ECNPs da Amostra B que tivessem distâncias no catálogo de Maciel (1484) e cujas idades foram estimadas pelo método 2. Há uma grande quantidade de ECNPs nesse caso e como o método 2 é mais robusto, esperamos ter uma noção geral do comportamento das distribuições.

A figura 4.9 apresenta os histogramas de contagens à esquerda, e os histogramas de frequência relativa à direita para as idades $t_{9}$ a $t_{12}$, admitindo a hipótese do movimento próprio nulo. A cor preta representa a distribuição de todas as ECNPs, como os histogramas vermelhos apresentados na figura 4.4; a cor vermelha representa apenas as ECNPs com idades acima de 0,3 Gano; a cor azul, ECNPs com idades acima de 0,5 Gano; e verde, ECNPs com idades acima de 0,7 Gano. Nota-se que grande parte das ECNPs, do primeiro intervalo, apresentam idades abaixo de 0,3 Gano. A diferença entre os histogramas de 
contagens preto e vermelho é muito grande para todas as idades, diminuindo notavelmente o pico proeminente do primeiro intervalo. A partir de $\simeq 0,5$ Gano, o pico da distribuição passa para o segundo intervalo de idade, exceto para $t_{11}$. A figura 4.10 apresenta os mesmos histogramas que no caso anterior, para a segunda hipótese do método 2: movimento próprio em ascensão reta aproximadamente igual ao em declinação. As variações no primeiro intervalo de idade e das distribuições relativas são semelhantes aos da figura 4.9 .

Portanto, a exclusão dos objetos muito jovens afeta a distribuição relativa no primeiro intervalo, mas a principal conclusão do método 2 não é afetada, isto é, a maior parte dos objetos analisados tem idades abaixo de 3 Gano aproximadamente, e o pico da distribuição encontra-se nesse intervalo de idade.

\subsubsection{Objetos com Determinações do Movimento Próprio}

A base de dados SIMBAD apresenta cerca de 50 NPs de nossa amostra com movimento próprio conhecido. Todas fazem parte da Amostra B e 33 estão contidas na Amostra A. É interessante estimarmos as idades dessas NPs aplicando o método 2, uma vez que conhecemos seus movimentos próprios verdadeiros, e verificarmos como as distribuições de idades se comportam. Apesar do número de NPs ser pequeno para um estudo estatístico, um comportamento mais geral pode ser percebido.

As 50 NPs apresentam velocidades radiais heliocêntricas no catálogo de Durand et aL (1998). Em torno de 42, possuem distâncias no catálogo de Maciell (1984) e 29 no catálogo de Stanghellini et al. (2008). Essas NPs estão apresentadas na tabela $\mathbb{B} .1$ do apêndice B. As três primeiras colunas $(1,2,3)$ mostram a identificação PN G (Acker et al., ए9.92), nome usual e identificação PK (Perek e Kohoutek, 1967) das nebulosas planetárias, respectivamente. A quarta coluna contém as velocidades radiais em $\mathrm{km} \mathrm{s}^{-1}$. A quinta e a sexta colunas apresentam as distâncias em kpc pela escala de Maciell (1984) e de Stanghellini et al. (20108), respectivamente. A sétima e a oitava colunas apresentam os dados do movimento próprio em ascensão reta e em delcinação em milisegundos de arco por ano, retirados da base de dados SIMBAD. A última coluna (9) designa qual amostra cada uma pertence.

Os cálculos foram feitos para as ECNPs que têm distâncias no catálogo de Maciell (1.984), pois além de possuir uma maior quantidade de objetos com determinações do movimento 

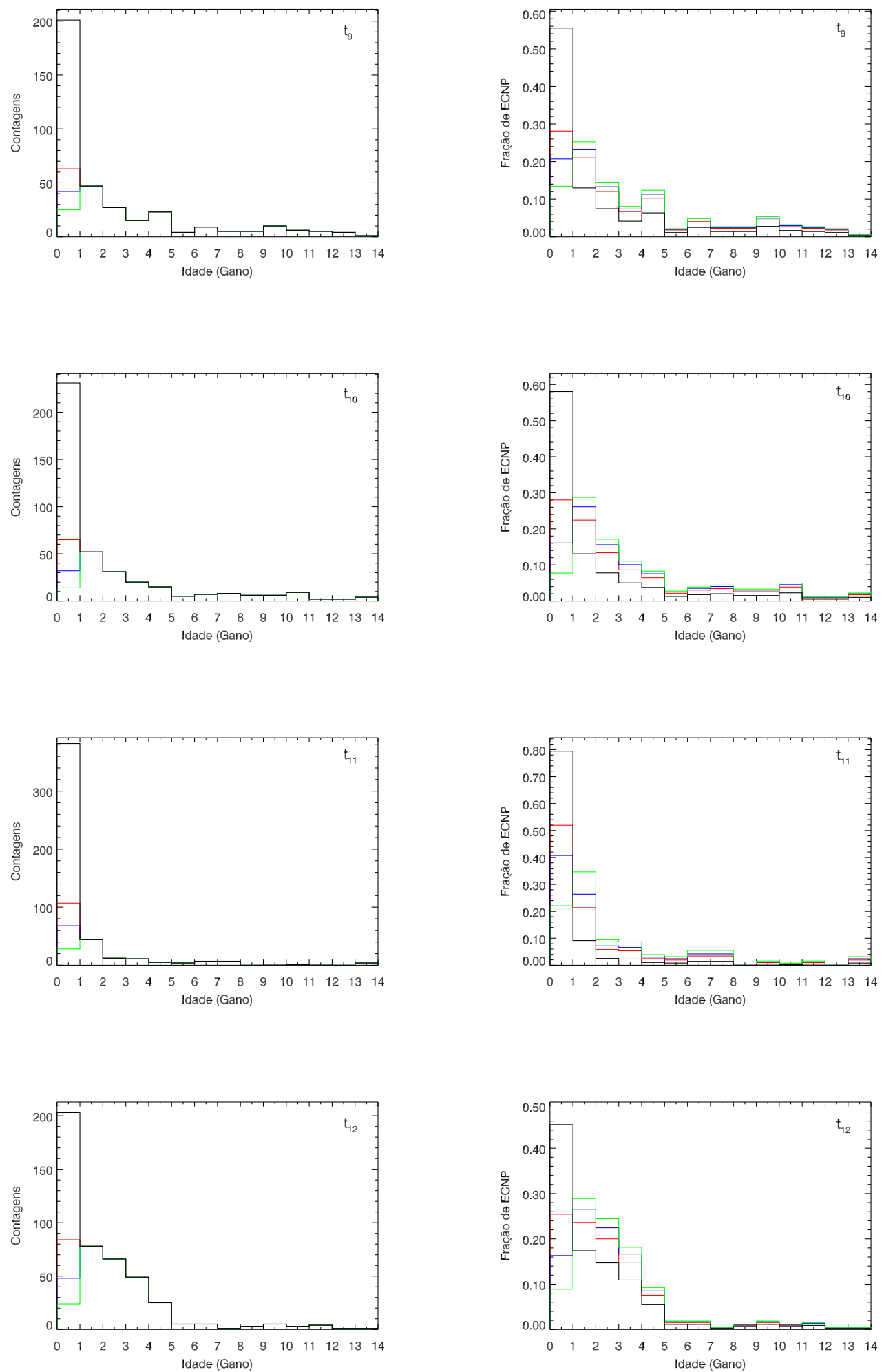

Figura 4.9: Distribuições de idades das ECNPs da Amostra B pelo método 2, admitindo a hipótese do movimento próprio nulo e atribuindo um limite inferior na relação idade-dispersão de velocidade. À esquerda, histogramas de contagens e à direita, histogramas de frequência relativa, para as idades $t_{9}$ a $t_{12}$. A cor preta representa todas as ECNPs; cor vermelha, ECNPs com idades acima de 0,3 Gano; cor azul, acima de 0,5 Gano; cor verde, acima de 0,7 Gano. 

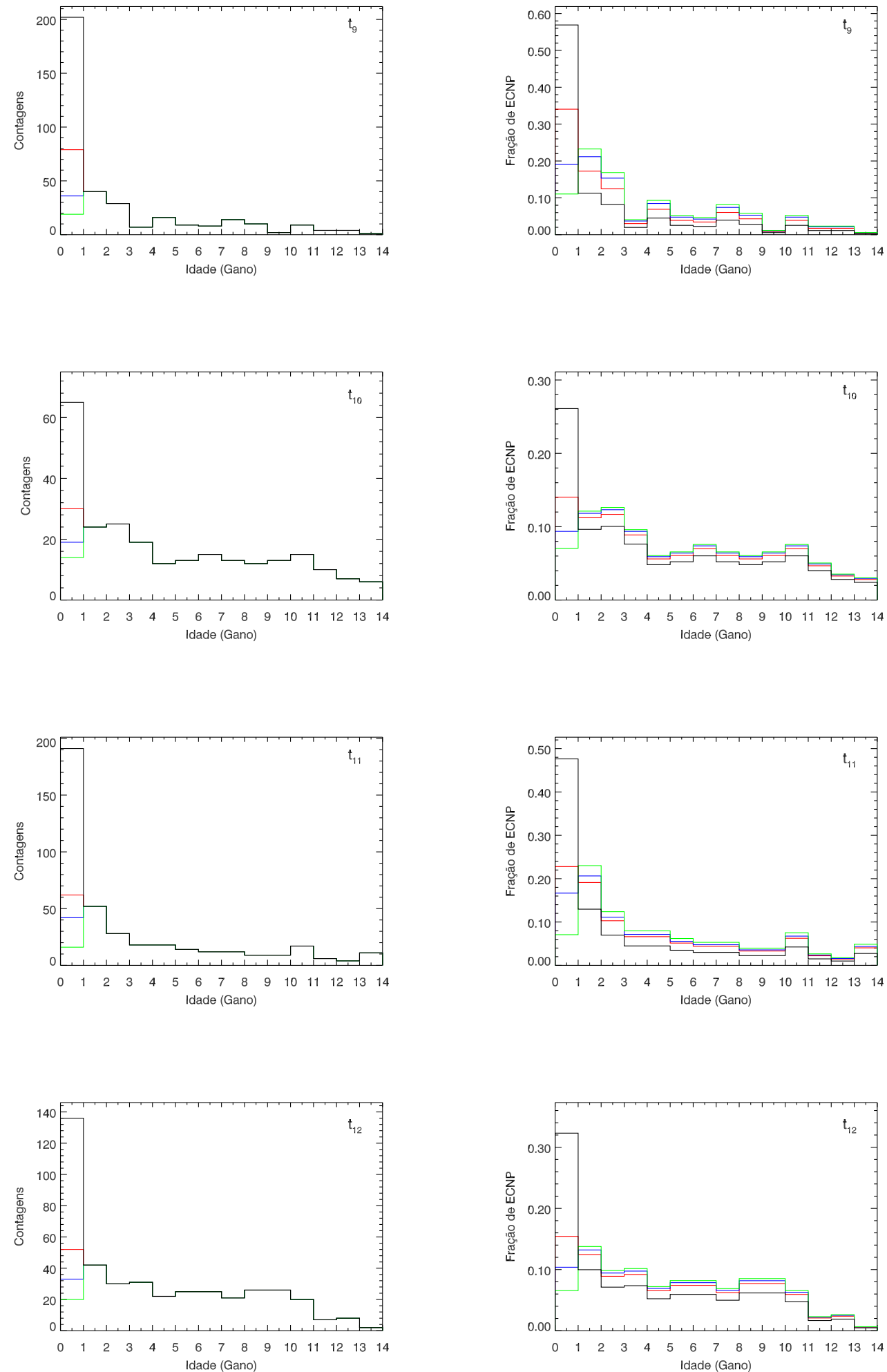

Figura 4.10: Distribuições de idades das ECNPs da Amostra B pelo método 2, admitindo a hipótese do movimento próprio em ascensão reta aproximadamente igual ao em declinação, e atribuindo um limite inferior na relação idade-dispersão de velocidade. À esquerda, histogramas de contagens e à direita, histogramas de frequência relativa, para as idades $t_{9}$ a $t_{12}$. A cor preta representa todas as ECNPs; cor vermelha, ECNPs com idades acima de 0,3 Gano; cor azul, acima de 0,5 Gano; cor verde, acima de 0,7 Gano. 
próprio, é considerada uma "escala curta", apresentando distâncias, em média, menores para as ECNPs. Em princípio, objetos mais próximos devem possuir determinações mais precisas para o movimento próprio. Então, as expressões $X, Y$ e $Z$, equação 3.9 puderam ser calculadas diretamente.

As componentes $U, V$ e $W$ foram calculadas pela equação 3.8 juntamente com a velocidade total $T$, equação उ.Tl e suas distribuições estão apresentadas na figura 4.71 . Duas NPs apresentaram velocidades muito grandes em uma das componentes. Como essas velocidades altas influenciavam a média aritmética das componentes, resolvemos excluí-las. Logo, as distribuições apresentadas possuem 40 ECNPs. Claramente as distribuições de velocidades não são simétricas, e provavelmente isso se deve à pequena amostra de NPs. A distribuição de $V$ apresenta mais velocidades negativas. A maior parte das ECNPs estão no intervalo de -80 a $80 \mathrm{~km} \mathrm{~s}^{-1}$. A distribuição de $T$ mostra um pico em torno de 60 a 80 $\mathrm{km} \mathrm{s}^{-1}$, diferente das distribuições anteriores que apresentaram esse pico em velocidades menores, $\simeq 20$ a $40 \mathrm{~km} \mathrm{~s}^{-1}$. A média aritmética de cada componente é: $\bar{U}=-6,09 \mathrm{~km} \mathrm{~s}^{-1}$, $\bar{V}=-20,04 \mathrm{~km} \mathrm{~s}^{-1}, \bar{W}=-1,78 \mathrm{~km} \mathrm{~s}^{-1}$, e $\bar{T}=90,07 \mathrm{~km} \mathrm{~s}^{-1}$.

Calculamos então as dispersões de velocidades pela equação [3.19 utilizando as médias encontradas. As idades foram estimadas como no caso anterior e suas distribuições estão apresentadas na figura 4. 2. Os histogramas $t_{9}$ a $t_{12}$ apresentam 25, 20, 23 e 37 ECNPs, respectivamente, as quais possuem idades fisicamente aceitáveis.

O número efetivo de objetos em cada histograma é pequeno, porém é possível concluir que as distribuições são semelhantes às encontradas anteriormente (figuras 4.2, 4.4, 4.6), ou seja, há um acumulo de objetos com idades abaixo de 3-4 Gano para as distribuições $t_{9}, t_{10}$ e $t_{12}$. Para a idade $t_{11}$, a distribuição não possui uma tendência clara.

Outra hipótese feita no método 2 foi admitir que a velocidade tangencial, em média, é semelhante à velocidade radial. Então calculamos as velocidades tangenciais das ECNPs com determinações do movimento próprio conforme dado pela equação [3.13. Verificando a distribuição dos módulos das velocidades radiais das ECNPs que apresentaram idades aceitáveis no método 2, percebemos que a grande maioria apresenta valores abaixo de $\left|V_{r}\right| \sim 100 \mathrm{~km} \mathrm{~s}^{-1}$, como exemplificado no histograma à esquerda na figura 4.13 para o caso da idade $t_{11}$ com a hipótese do movimento próprio não-nulo. Logo, comparamos os módulos das velocidades radiais $\left(\left|V_{r}\right|\right)$ e tangenciais $\left(\left|V_{t}\right|\right)$ das ECNPs até esse valor, 

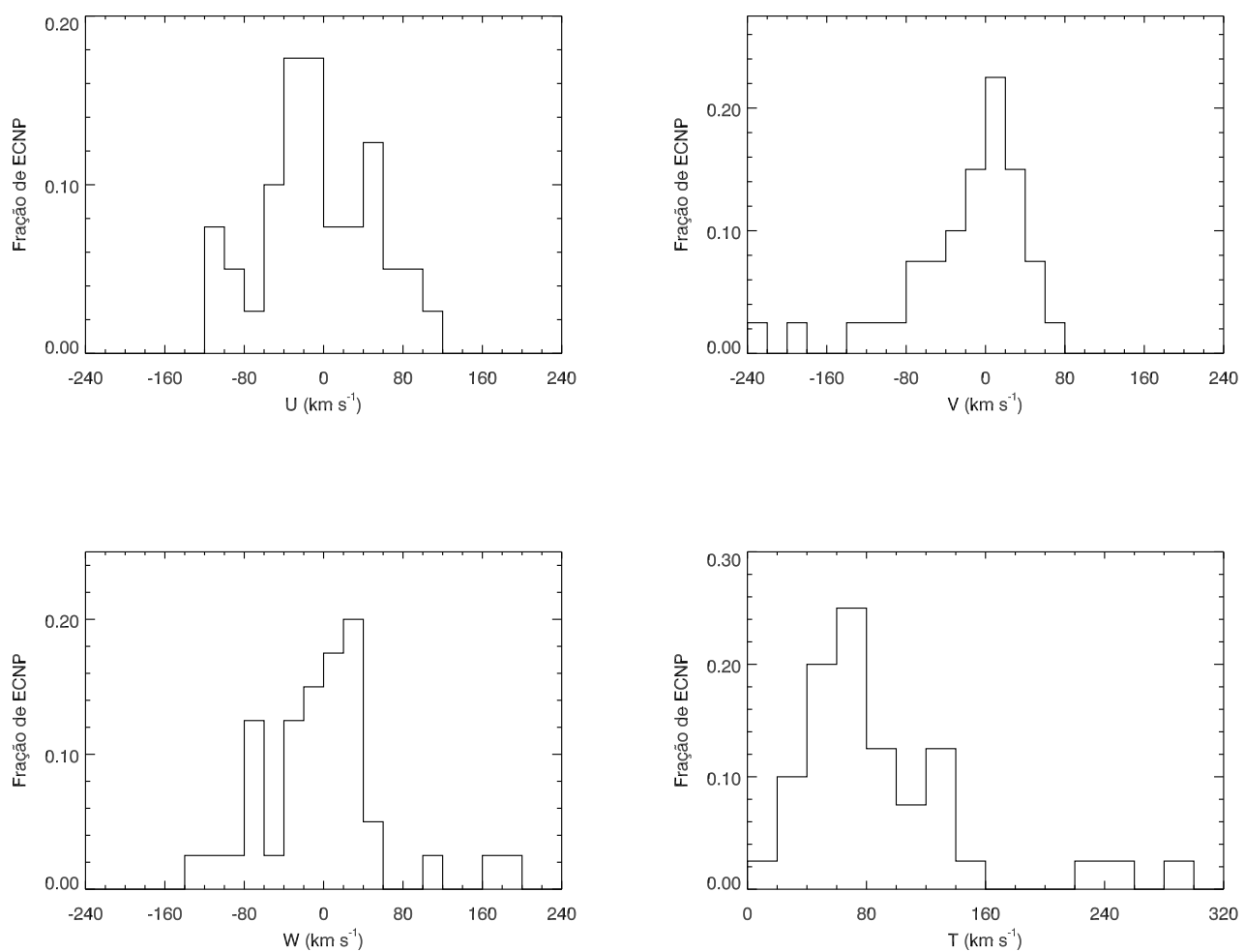

Figura 4.11: Distribuições das velocidades $U, V, W$ e $T$ pelo método 2 para ECNPs com determinações do movimento próprio. As distâncias foram retiradas do catálogo de Maciel ([1984).

como apresentado no lado direito da figura 4.13. A linha sólida indica o acordo perfeito, isto é, a relação 1:1. O gráfico apresenta 27 ECNPs, embora seja um número pequeno, aparentemente a hipótese feita é razoável.

\subsection{Incertezas}

As incertezas das distribuições de idades, formalmente, provêm das incertezas de todos os parâmetros envolvidos. Quanto às distâncias adotadas, essas são difíceis de estimar, pois envolvem as incertezas das propriedades dos objetos calibradores. A escala de distância de Maciel (1.984) apresenta erros entre 50\% a 70\%, enquanto que a de Stanghellini et al. (20)(18) apresenta erros em torno de 30\%. Por isso, adotamos essas duas escalas que foram obtidas por diferentes hipóteses e que não concordam em toda a extensão de distância, como mostrado na figura [2.4. Porém, não foi notada diferença significativa entre as dis- 

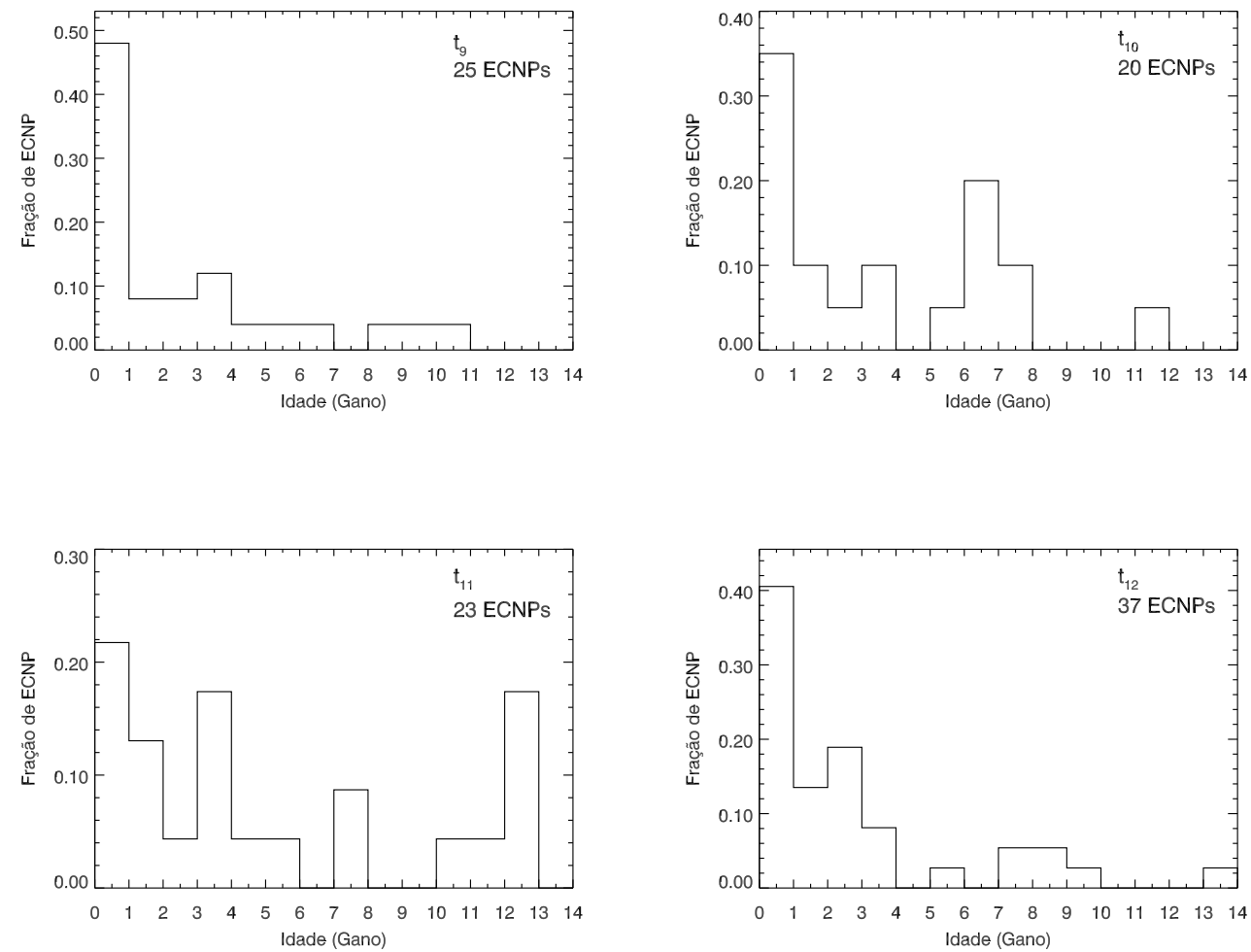

Figura 4.12: Distribuições de idades pelo método 2 para ECNPs com determinações do movimento próprio.

tribuições de idades estimadas com ambas as escalas, o que pode ser visto ao compararmos os histogramas $t_{1}$ a $t_{4}$ com os $t_{5}$ a $t_{8}$ do método 1 , e os histogramas $t_{9}$ a $t_{12}$ com os $t_{13}$ a $t_{16}$ do método 2. Portanto, as escalas de distância não devem causar incertezas expressivas nas distribuições de idades.

Como apresentado na seção [2.31, cerca de 80\% das ECNPs apresentam erros na velocidade radial heliocêntrica menor ou igual a $10 \mathrm{~km} \mathrm{~s}^{-1}$, o que também não deve ter um efeito importante na incerteza das distribuições de idades. As velocidades de rotação derivadas para a curva de rotação de Clemens (1985) apresentam desvios quadráticos médios em torno de $20 \mathrm{~km} \mathrm{~s}^{-1}$, enquanto que para as NPs esse valor é maior, cerca de $50 \mathrm{~km} \mathrm{~s}^{-1}$ (Maciel e Lago, 20(05). Não foi notada diferença representativa entre as distribuições de idades derivadas com a utilização de ambas as curvas de rotação teóricas, como pode ser visto na comparação dos histogramas $t_{1}$ e $t_{2}$ com $t_{3}$ e $t_{4}$ para a escala de distância de Maciell (1984), e $t_{5}$ e $t_{6}$ com $t_{7}$ e $t_{8}$ para a escala de distância de Stanghellini et al. (2008), no método 1. Entretanto, o método 1 não leva em conta desvios do movimento de rotação nem a 

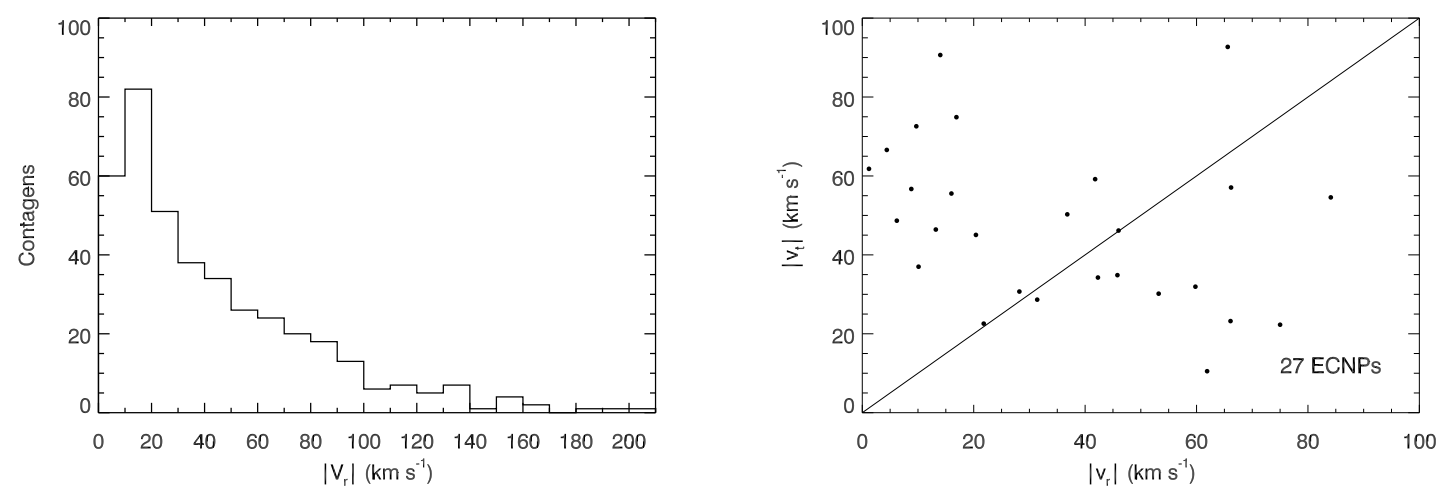

Figura 4.13: Lado esquerdo: distribuição do módulo da velocidade radial heliocêntrica das ECNPs com idades $t_{11}$ e com a hipótese de movimento próprio não-nulo. Lado direito: comparação entre o módulo da velocidade radial e o módulo da velocidade tangencial para as ECNPs com determinações do movimento próprio.

possibilidade de que outros efeitos, além da idade, possam influir na diferença entre as velocidades de rotação. O fato de que os resultados dos dois métodos são semelhantes sugere que essas fontes de incerteza não são muito importantes no que se refere às distribuições de idades.

As incertezas das distribuições de idades podem ser calculadas propagando os erros de cada grandeza, entretanto é provável que a maior fonte de incertezas seja introduzida pela determinação das idades e das dispersões de velocidades da relação idade-dispersão de velocidade do levantamento Geneva-Copenhagen. As idades usadas para derivar a relação possuem erros abaixo de 25\%, o que produz uma incerteza máxima para uma estrela de 14 Gano em torno de 3,5 Gano. A média no cálculo de 25\% nas idades das ECNPs contabilizadas em cada distribuição resulta valores entre 0,5 a 1,3 Gano. Como a maioria dos objetos possuem idades abaixo de 5 Gano, podemos adotar um valor aproximado de 1,2 Gano para a incerteza das distribuições do método 2, e um valor um pouco mais alto para o método 1, da ordem de 2 Gano, semelhante aos valores dos métodos baseados nas abundâncias (Maciel et an, [2010). 
Capítulo 5

\section{Conclusões e Perspectivas}

\subsection{Conclusões}

Neste trabalho, adotamos o método de idades estatísticas baseado na relação idadedispersão de velocidade para derivar as distribuições de idades de duas amostras de ECNPs Galácticas. Essa relação descreve o aquecimento do disco da Galáxia devido, provavelmente, às interações entre objetos massivos com as estrelas.

Os métodos consistem em determinar as dispersões de velocidade das componentes da velocidade espacial de cada ECNP, uma vez que elas são diretamente correlacionadas com a idade do objeto. O método 1 considera que a diferença entre a velocidade de rotação da ECNP e da velocidade predita pela curva de rotação Galáctica em sua posição é inteiramente devido ao aquecimento que a estrela sofreu, indicando assim sua idade. Porém, outros processos podem estar contidos nessa diferença de velocidade, tais como o desvio de uma rotação circular. O método 2 baseia-se em calcular diretamente as componentes da velocidade espacial, porém com a necessidade de algumas hipóteses quanto ao movimento próprio da estrela, pois esse parâmetro é apenas conhecido para uma pequena quantidade de ECNPs.

Como é um estudo estatístico, uma estrela que apresenta uma pequena dispersão não necessariamente é um objeto jovem ou vice-versa. Seabroke e Gilmore (2007) analisaram cada intervalo de idade da relação idade-dispersão de velocidade do levantamento GenevaCopenhagen, construindo histogramas das componentes das velocidades para cada um. As distribuições de velocidade dos objetos mais jovens apresentam uma concentração maior em velocidades baixas, mas há uma fração pequena desses objetos com velocidades altas, e a medida que a idade dos objetos aumenta, as distribuições alargam-se para velocidades 
maiores. Como dependemos dessa relação para a estimativa das idades, ela é aplicada diretamente em nossos métodos, ou seja, todos as ECNPs de baixa dispersão resultam em objetos jovens, e as de alta dispersão, em objetos com idades mais avançadas. Portanto, as idades calculadas devem ser consideradas como um valor limite, uma vez que foram derivadas de um parâmetro de dispersão, o que representa a variabilidade dentro de um certo intervalo em estatística.

Ambos os métodos apresentam distribuições muito semelhantes, embora sejam baseados em hipóteses distintas e utilizem parâmetros diferentes, como escalas de distâncias, curvas de rotação e dispersões das componentes da velocidade espacial. Os principais resultados são:

- O comportamento geral notado em todas as distribuições de idades encontradas é que a maioria das ECNPs apresenta idades abaixo de 5 Gano, e o pico da distribuição encontra-se entre 0 e 3 Gano.

- Não há diferença representativa entre as distribuições de idades do método 1 usando a dispersão da componente $V$ ou da velocidade total $T$, para ambas as escalas de distância e amostras.

- Não há diferença significativa entre as distribuições de idades pelas dispersões em $U, V, W$ e $T$ no método 2. Segundo a análise de Seabroke e Gilmore (2007), o aquecimento do disco é melhor percebido pela componente $W$ da velocidade espacial. Portanto, as distribuições de idades derivadas para essa componente devem ser mais acurada.

- A hipótese do movimento próprio nulo do método 2 apresenta uma quantidade muito maior de ECNPs em idades baixas, até 3 Gano. Enquanto que a hipótese do movimento próprio não-nulo mostra ECNPs mais distribuídas em outros intervalos de idade.

- Apesar de a Amostra A conter menos da metade dos objetos com parâmetros necessários disponíveis que a Amostra B, ela parece ser representativa da população de ECNPs, uma vez que suas distribuições de idades são muito semelhantes com as da Amostra B. 
- O método 2 parece ser mais plausível que o método 1, pois além de calcular diretamente as componentes da velocidade espacial com hipóteses mais razoáveis, também apresenta uma maior quantidade de ECNPs com idades aceitáveis fisicamente.

- As incertezas das distribuições devem provir principalmente da relação idade-dispersão de velocidade adotada, sendo aproximadamente 1,2 Gano para o método 2 e um pouco maior, da ordem de 2 Gano, para o método 1.

- A variação do sinal do movimento próprio adotado não influência expressivamente no comportamento geral das distribuições de idades.

- A adoção de um limite inferior de idade para aplicar a relação idade-dispersão de velocidade tende a modificar o pico da distribuição, deslocando-o para idades um pouco maiores, mas ainda assim é mantida a conclusão de que a maior parte dos objetos tem idades abaixo de 5 Gano, com um pico abaixo de 3 Gano.

- As distribuições de idades derivadas para as ECNPs com determinações do movimento próprio são semelhantes às distribuições obtidas com as hipóteses do método 2. A hipótese sobre a velocidade tangencial se mostra bastante razoável.

As distribuições de idades encontradas aqui assemelham-se com as derivadas pela relação idade-massa (N/O), para o caso B da relação idade-massa da estrela na SP, em Maciel et aL. (2010), explicado na subseção ․2.2.3. Concordando, também, com a distribuição de idades estimada diretamente da distribuição de massas empíricas das ECNPs no mesmo trabalho (subseção प.2.2.4). As incertezas dos nossos métodos são menores que as encontradas pelos métodos desenvolvidos em Maciel et al. (2010).

\subsection{Perspectivas Futuras}

Dos dois métodos analisados neste trabalho, o método 2 produz resultados mais corretos, uma vez que suas hipóteses podem ser verificadas com maior exatidão. Considerando que as distâncias e as velocidades radiais aqui utilizadas são relativamente precisas, seria interessante aplicar esse método utilizando os movimentos próprios reais das ECNPs. A médio prazo isto deverá ser possível com os resultados previstos do projeto GAIA. 
Uma outra alternativa para a falta desse parâmetro e da velocidade tangencial seria a aplicação da técnica de Decomposição em Valores Singulares (em inglês singular value decomposition), como em Branham (2010), o que pode também confirmar os valores obtidos com as hipóteses do método 2 .

As distribuições de idades de ECNPs aqui estimadas podem auxiliar na compreensão da evolução química e dinâmica da Galáxia, particularmente no estudo da evolução temporal de seus gradientes radiais de abundâncias, como preliminarmente analisado em Maciel et al. (2011b). Além disso, seria também relevante no entendimento da evolução e nas propriedades das estrelas de massa baixa e intermediária. 


\section{Referências Bibliográficas}

Acker A., Marcout J., Ochsenbein F., Stenholm B., Tylenda R., Schohn C., The Strasbourg-ESO catalogue of Galactic planetary nebulae. Parts I, II., 1992

Bahcall J. N., Piran T., Stellar collapses in the galaxy, ApJ, 1983, vol. 267, p. L77

Barbanis B., Woltjer L., Orbits in spiral galaxies and the velocity dispersion of population I stars, ApJ, 1967, vol. 150, p. 461

Barnes S. A., Ages for illustrative field stars using gyrochronology: viability, limitations, and errors, ApJ, 2007, vol. 669, p. 1167

Beaulieu S., Dynamics of the galactic bulge using planetary nebulae, Australian National University, 1996, Tese de Doutorado

Blöcker T., Stellar evolution of low- and intermediate-mass stars. II. Post-AGB evolution., A\&A, 1995, vol. 299, p. 755

Blöcker T., Evolution on the AGB and beyond: on the formation of H-deficient post-AGB stars, Ap\&SS, 2001, vol. 275, p. 1

Boesgaard A. M., Tripicco M. J., Lithium in early F dwarfs, ApJ, 1986, vol. 303, p. 724

Branham Jr. R. L., Kinematics and velocity ellipsoid of the F giants, MNRAS, 2010, vol. 409, p. 1269

Cahn J. H., Kaler J. B., The distances and distribution of planetary nebulae, ApJS, 1971, vol. 22 , p. 319 
Cahn J. H., Kaler J. B., Stanghellini L., A catalogue of absolute fluxes and distances of planetary nebulae, A\&AS, 1992, vol. 94, p. 399

Carlberg R. G., Sellwood J. A., Spiral instabilities and disk heating. In Internal Kinematics and Dynamics of Galaxies, vol. 100 of IAU Symposium, 1983, p. 127

Carlberg R. G., Sellwood J. A., Dynamical evolution in galactic disks, ApJ, 1985, vol. 292, p. 79

Cazetta J. O., Maciel W. J., Distances of Galactic planetary nebulae based on a relationship between the central star mass and the N/O abundance, Rev. Mexicana Astron. Astrofis., 2000, vol. 36, p. 3

Clemens D. P., Massachusetts-Stony Brook Galactic plane CO survey - The Galactic disk rotation curve, ApJ, 1985, vol. 295, p. 422

Daub C. T., A statistical survey of local planetary nebulae, ApJ, 1982, vol. 260, p. 612

de Freitas Pacheco J. A., Oxygen, sulfur, argon, and the chemical evolution of the galactic disk, ApJ, 1993, vol. 403, p. 673

De Simone R., Wu X., Tremaine S., The stellar velocity distribution in the solar neighbourhood, MNRAS, 2004, vol. 350, p. 627

Dopita M. A., Hua C. T., Southern emission-line flux standards, ApJS, 1997, vol. 108, p. 515

Durand S., Acker A., Ziljlstra A., The kinematics of 867 galactic planetary nebulae, A\&AS, 1998, vol. 132, p. 13

Edvardsson B., Andersen J., Gustafsson B., Lambert D. L., Nissen P. E., Tomkin J., The chemical evolution of the Galactic disk - part one - analysis and results, A\&A, 1993, vol. 275 , p. 101

ESA The Hipparcos and Tycho Catalogues (ESA 1997), VizieR Online Data Catalog, 1997, vol. 1239 , p. 0 
Famaey B., Jorissen A., Luri X., Mayor M., Udry S., Dejonghe H., Turon C., Local kinematics of $\mathrm{K}$ and $\mathrm{M}$ giants from CORAVEL/Hipparcos/Tycho-2 data. Revisiting the concept of superclusters, A\&A, 2005, vol. 430, p. 165

Faundez-Abans M., Maciel W. J., The classification of planetary nebulae, A\&A, 1987, vol. 183 , p. 324

Fux R., Order and chaos in the local disc stellar kinematics induced by the Galactic bar, A\&A, 2001, vol. 373, p. 511

Gesicki K., Zijlstra A. A., White dwarf masses derived from planetary nebula modelling, A\&A, 2007, vol. 467, p. L29

Girardi L., Bressan A., Bertelli G., Chiosi C., Evolutionary tracks and isochrones for lowand intermediate-mass stars: From 0.15 to $7 \mathrm{M}_{\text {sun }}$, and from $\mathrm{Z}=0.0004$ to 0.03 , A\&AS, 2000, vol. 141, p. 371

Hajian A. R., Distances to planetary nebulae. In Planetary Nebulae in our Galaxy and Beyond, vol. 234 of IAU Symposium, 2006, p. 41

Hänninen J., Flynn C., Simulations of the heating of the Galactic stellar disc, MNRAS, 2002, vol. 337, p. 731

Høg E., Fabricius C., Makarov V. V., Urban S., Corbin T., Wycoff G., Bastian U., Schwekendiek P., Wicenec A., The Tycho-2 catalogue of the 2.5 million brightest stars, A\&A, 2000, vol. 355, p. L27

Holmberg J., Nordström B., Andersen J., The Geneva-Copenhagen survey of the Solar neighbourhood II. New uvby calibrations and rediscussion of stellar ages, the G dwarf problem, age-metallicity diagram, and heating mechanisms of the disk, A\&A, 2007, vol. 475 , p. 519

Holmberg J., Nordström B., Andersen J., The Geneva-Copenhagen survey of the solar neighbourhood. III. Improved distances, ages, and kinematics, A\&A, 2009, vol. 501, p. 941 
Idiart T. P., Maciel W. J., Costa R. D. D., Chemical evolution of the Small Magellanic Cloud based on planetary nebulae, A\&A, 2007, vol. 472, p. 101

Jørgensen B. R., Lindegren L., Determination of stellar ages from isochrones: Bayesian estimation versus isochrone fitting, A\&A, 2005, vol. 436, p. 127

Kerr F. J., Lynden-Bell D., Review of galactic constants, MNRAS, 1986, vol. 221, p. 1023

Kippenhahn R., Weigert A., Stellar structure and evolution. Springer-Verlag, 1994

Kohoutek L., Pauls R., Planetary nebulae in the direction of the galactic bulge. I. New radial velocities based on image-tube spectra., A\&AS, 1995, vol. 111, p. 493

Kraan-Korteweg R. C., Fairall A. P., Woudt P. A., van de Steene G. C., Two new planetary nebulae discovered in a galaxy search in the southern Milky Way., A\&A, 1996, vol. 315, p. 549

Kwok S., The origin and evolution of planetary nebulae. Cambridge University Press, 2000

Lacey C. G., The influence of massive gas clouds on stellar velocity dispersions in galactic discs, MNRAS, 1984, vol. 208, p. 687

Lacey C. G., Ostriker J. P., Massive black holes in galactic halos?, ApJ, 1985, vol. 299, p. 633

Lang K. R., Astrophysycal formulae. Springer-Verlag, 1978

Maciel W. J., Distances of planetary nebulae. II, A\&AS, 1981, vol. 44, p. 123

Maciel W. J., A catalogue of distances of planetary nebulae, A\&AS, 1984, vol. 55, p. 253

Maciel W. J., Galactic distribution radial velocities and masses of PN. In Planetary Nebulae, vol. 131 of IAU Symposium, 1989, p. 73

Maciel W. J., Nebular abundances and the chemical evolution of the Galaxy. In Elements and the Cosmos, 1992, p. 210

Maciel W. J., Introdução à estrutura e evolução estelar. Edusp, 1999 
Maciel W. J., Helium contamination from the progenitor stars of planetary nebulae: The He/H radial gradient and the $\Delta \mathrm{Y} / \Delta \mathrm{Z}$ enrichment ratio, Ap\&SS, 2001, vol. 277, p. 545

Maciel W. J., Costa R. D. D., Idiart T. E. P., Age distribution of the central stars of galactic disk planetary nebulae, A\&A, 2010, vol. 512, p. A19

Maciel W. J., Costa R. D. D., Uchida M. M. M., An estimate of the time variation of the $\mathrm{O} / \mathrm{H}$ radial gradient from planetary nebulae, A\&A, 2003, vol. 397, p. 667

Maciel W. J., Köppen J., Abundance gradients from disk planetary nebulae: O, Ne, S, and AR, A\&A, 1994, vol. 282, p. 436

Maciel W. J., Lago L. G., A new determination of the rotation curve from Galactic disk planetary nebulae, Rev. Mexicana Astron. Astrofis., 2005, vol. 41, p. 383

Maciel W. J., Lago L. G., Costa R. D. D., An estimate of the time variation of the abundance gradient from planetary nebulae. II. Comparison with open clusters, cepheids and young objects, A\&A, 2005, vol. 433, p. 127

Maciel W. J., Lago L. G., Costa R. D. D., An estimate of the time variation of the abundance gradient from planetary nebulae. III. O, S, Ar, and Ne: a comparison of PN samples, A\&A, 2006, vol. 453, p. 587

Maciel W. J., Pottasch S. R., Distances of planetary nebulae, A\&A, 1980, vol. 88, p. 1

Maciel W. J., Quireza C., Abundance gradients in the outer galactic disk from planetary nebulae, A\&A, 1999, vol. 345, p. 629

Maciel W. J., Rodrigues T. S., Costa R. D. D., Kinematic ages of the central stars of planetary nebulae, Rev. Mexicana Astron. Astrofis., 2011a, vol. 47, p. 401

Maciel W. J., Rodrigues T. S., Costa R. D. D., Radial abundance gradients from planetary nebulae and young objects: age effects. In Planetary Nebulae - An Eye to the Future, vol. 283 of IAU Symposium, submitted, 2011b

Madej J., Należyty M., Althaus L. G., Mass distribution of DA white dwarfs in the first data release of the Sloan Digital Sky Survey, A\&A, 2004, vol. 419, p. L5 
Malkin Z., The current best estimate of the Galactocentric distance of the Sun based on comparison of different statistical techniques, ArXiv e-prints, 2012

Mamajek E. E., Soderblom D. R., Wyse R. F. G., eds. 2009. The ages of stars vol. 258 of IAU Symposium

Mihalas D., Binney J., Galactic astronomy. Freeman, 1981

Milne D. K., Radio observations at $5 \mathrm{GHz}$ of southern planetary nebula II, A\&AS, 1979, vol. 36 , p. 227

Milne D. K., Aller L. H., Radio observations at $5 \mathrm{GHz}$ of southern planetary nebulae., A\&A, 1975, vol. 38, p. 183

Nissen P. E., Abundance distribution in Galactic stellar populations. In Elements and the Cosmos, 1992, p. 110

Nordström B., Signatures of heating processes in the Galactic thin disk. In IAU Symposium, vol. 258 of IAU Symposium, 2009, p. 31

Nordström B., Mayor M., Andersen J., Holmberg J., Pont F., Jørgensen B. R., Olsen E. H., Udry S., Mowlavi N., The Geneva-Copenhagen survey of the Solar neighbourhood. Ages, metallicities, and kinematic properties of $14000 \mathrm{~F}$ and G dwarfs, A\&A, 2004, vol. 418, p. 989

Peimbert M., Chemical abundances in planetary nebulae. In Planetary Nebulae, vol. 76 of IAU Symposium, 1978, p. 215

Peimbert M., Serrano A., On the helium and nitrogen enrichment of the interstellar medium by planetary nebulae, Rev. Mexicana Astron. Astrofis., 1980, vol. 5, p. 9

Peimbert M., Torres-Peimbert S., Type I planetary nebulae. In Planetary Nebulae, vol. 103 of IAU Symposium, 1983, p. 233

Perek L., Kohoutek L., Catalogue of galactic planetary nebulae., Publ. House Czech. Acad. Sci., 1967 
Quillen A. C., Garnett D. R., The saturation of disk heating in the Solar neighborhood and evidence for a merger 9 Gyr ago. In Galaxy Disks and Disk Galaxies, vol. 230 of Astronomical Society of the Pacific Conference Series, 2001, p. 87

Quinn P. J., Hernquist L., Fullagar D. P., Heating of galactic disks by mergers, ApJ, 1993, vol. 403 , p. 74

Quireza C., Rocha-Pinto H. J., Maciel W. J., Bayesian posterior classification of planetary nebulae according to the Peimbert types, A\&A, 2007, vol. 475, p. 217

Reiners A., Basri G., A volume-limited sample of 63 M7-M9.5 dwarfs. I. Space motion, kinematic age, and lithium, ApJ, 2009, vol. 705, p. 1416

Rocha-Pinto H. J., Maciel W. J., Scalo J., Flynn C., Chemical enrichment and star formation in the Milky Way disk. I. Sample description and chromospheric age-metallicity relation, A\&A, 2000, vol. 358, p. 850

Roman N. G., A correlation between the spectroscopic and dynamical characteristics of the late F - and early G - type stars., ApJ, 1950, vol. 112, p. 554

Roman N. G., The spectra of the bright stars of types F5-K5., ApJ, 1952, vol. 116, p. 122

Salasnich B., Girardi L., Weiss A., Chiosi C., Evolutionary tracks and isochrones for alpha -enhanced stars, A\&A, 2000, vol. 361, p. 1023

Seabroke G. M., Gilmore G., Revisiting the relations: Galactic thin disc age-velocity dispersion relation, MNRAS, 2007, vol. 380, p. 1348

Sellwood J. A., Carlberg R. G., Spiral instabilities provoked by accretion and star formation, ApJ, 1984, vol. 282, p. 61

Soderblom D. R., Kinematics of chromospherically active late-type dwarfs in the solar neighborhood, AJ, 1990, vol. 100, p. 204

Soderblom D. R., Some problems in studying the ages of stars. In IAU Symposium, vol. 258 of IAU Symposium, 2009, p. 1

Soderblom D. R., The ages of stars, ARA\&A, 2010, vol. 48, p. 581 
Spitzer Jr. L., Schwarzschild M., The possible influence of interstellar clouds on stellar velocities., ApJ, 1951, vol. 114, p. 385

Spitzer Jr. L., Schwarzschild M., The possible influence of interstellar clouds on stellar velocities. II., ApJ, 1953, vol. 118, p. 106

Spyromilio J., Wavelengths of the $[\mathrm{NII}]{ }^{3} \mathrm{P}-{ }^{1} \mathrm{D}$ forbidden lines, MNRAS, 1995, vol. 277, p. L59

Stanghellini L., Shaw R. A., Villaver E., The Magellanic Cloud calibration of the Galactic planetary nebula distance scale, ApJ, 2008, vol. 689, p. 194

Stasińska G., Abundance determinations in H II regions and planetary nebulae. In Cosmochemistry. The melting pot of the elements, 2004, p. 115

Toth G., Ostriker J. P., Galactic disks, infall, and the global value of Omega, ApJ, 1992, vol. 389 , p. 5

Twarog B. A., The chemical evolution of the solar neighborhood. II - The age-metallicity relation and the history of star formation in the galactic disk, ApJ, 1980, vol. 242, p. 242

van Leeuwen F., Validation of the new Hipparcos reduction, A\&A, 2007, vol. 474, p. 653

Vassiliadis E., Wood P. R., Post-asymptotic giant branch evolution of low- to intermediatemass stars, ApJS, 1994, vol. 92, p. 125

Wielen R., The diffusion of stellar orbits derived from the observed age-dependence of the velocity dispersion, A\&A, 1977, vol. 60, p. 263

Zijlstra A. A., Acker A., Walsh J. R., Radial velocities of planetary nebulae towards the Galactic bulge, A\&AS, 1997, vol. 125, p. 289 
Apêndice 

Apêndice A

\section{Amostra de Nebulosas Planetárias}

A tabela A.1] apresenta todos os objetos das amostras escolhidas. As três primeiras colunas $(1,2,3)$ mostram a identificação PN G (Acker et al., 19922), nome usual e identificação PK (Perek e Kohoutek, 1967) das nebulosas planetárias, respectivamente. A quarta coluna contém a velocidade radial heliocêntrica $\mathrm{em} \mathrm{km} \mathrm{s}^{-1}$ retirada do catálogo de Durand et al. ([1998). A quinta e a sexta colunas estão as distâncias heliocêntricas em kpc obtidas do catálogo de Maciel (1484) ( $\left.d_{\mathrm{M} 84}\right)$ e Stanghellini et al. (2008) ( $\left.d_{\mathrm{SSV} 08}\right)$. A sétima e a oitava colunas estão a ascensão reta $(\alpha)$ e a declinação $(\delta)$ para a época 1950. A última coluna (9) designa qual amosta cada nebulosa planetária pertence.

Tabela A.1 - Amostra de nebulosas planetárias.

\begin{tabular}{ccccccccc}
\hline \hline PN G & Nome & PK & $V_{r}$ (hel) & $d_{\text {M } 84}$ & $d_{\text {SSV08 }}$ & $\alpha(1950)$ & $\delta(1950)$ & Amostra \\
\hline $000.0-06.8$ & H $1-62$ & $359-061$ & $-86,6$ & & & 181001,9 & $-322033,9$ & B \\
$000.1+04.3$ & H $1-16$ & $000+042$ & 55,6 & & & 172616,7 & $-262344,8$ & B \\
$000.1+17.2$ & PC 12 & $000+171$ & $-60,3$ & 6,1 & 14,4 & 164058,4 & $-185139,2$ & B \\
$000.1-01.1$ & M 3-43 & $000-011$ & $-7,3$ & 0,9 & 8,5 & 174712,9 & $-292429,6$ & B \\
$000.1-02.3$ & B 3-10 & $000-022$ & $-109,0$ & & & 175208,3 & $-295708,7$ & B \\
$000.1-05.6$ & H 2-40 & $000-051$ & $-54,3$ & 6,4 & & 180515,7 & $-313704,7$ & B \\
$000.1-08.0$ & SB1 & $000-081$ & $-54,0$ & & & 181532,0 & $-324913,1$ & B \\
$000.2-01.9$ & M 2-19 & $000-015$ & $-61,2$ & 1,0 & 8,3 & 175033,7 & $-294312,1$ & B \\
$000.2-04.6$ & Wray16-363 & $000-043$ & 121,4 & & & 180130,1 & $-310301,0$ & B \\
$000.3+06.9$ & Trz 41 & $000+062$ & 5,8 & & & 171717,8 & $-244853,1$ & B \\
$000.3+12.2$ & IC 4634 & $000+121$ & $-36,3$ & 2,5 & 3,9 & 165834,0 & $-214514,1$ & A, B \\
$000.3-02.8$ & M 3-47 & $000-025$ & $-16,0$ & & & 175430,9 & $-300212,9$ & B \\
$000.3-04.6$ & M 2-28 & $000-041$ & $-29,9$ & & 9,5 & 180148,8 & $-305832,4$ & B \\
$000.4-01.9$ & M 2-20 & $000-016$ & 58,7 & & & 175113,7 & $-293536,8$ & B \\
$000.4-02.9$ & M 3-19 & $000-026$ & 141,7 & 2,2 & 7,9 & 175507,0 & $-300025,0$ & B \\
$000.5-01.6$ & Al 2-Q & $000-017$ & $-67,0$ & & & 175013,8 & $-291633,0$ & B \\
$000.5-03.1$ & KFL 1 & $000-032$ & $-52,8$ & & & 175603,1 & $-300236,9$ & B \\
\hline
\end{tabular}

Continua na próxima página... 
Apêndice A. Amostra de Nebulosas Planetárias

Tabela A.1 - Continuação

\begin{tabular}{|c|c|c|c|c|c|c|c|c|}
\hline PN G & Nome & $\mathrm{PK}$ & $V_{r}($ hel $)$ & $d_{\mathrm{M} 84}$ & $d_{\mathrm{SSV} 08}$ & $\alpha(1950)$ & $\delta(1950)$ & Amostra \\
\hline $000.5-05.3$ & SB2 & 000-05 2 & 3,0 & & & 180520,6 & $-310722,0$ & B \\
\hline $000.6-02.3$ & H $2-32$ & $000-023$ & 128,7 & & & 175312,6 & $-293744,0$ & B \\
\hline $000.7+03.2$ & He $2-250$ & $000+031$ & $-175,1$ & 2,7 & & 173147,7 & $-263400,6$ & $\mathrm{~A}, \mathrm{~B}$ \\
\hline $000.7+04.7$ & H $2-11$ & $000+041$ & 10,2 & & & 172620,2 & $-254647,1$ & B \\
\hline $000.7-02.7$ & M 2-21 & $000-024$ & $-164,5$ & & 10,2 & 175457,6 & $-294405,0$ & $\mathrm{~B}$ \\
\hline $000.7-03.7$ & M 3-22 & $000-031$ & $-56,2$ & 3,0 & 7,7 & 175906,5 & $-301428,5$ & B \\
\hline $000.7-06.1$ & SB3 & $000-062$ & 68,0 & & & 180900,1 & $-312044,4$ & B \\
\hline $000.7-07.4$ & M 2-35 & $000-071$ & $-30,1$ & & & 181421,8 & $-315756,7$ & B \\
\hline $000.8-07.6$ & H $2-46$ & $000-072$ & 47,9 & & & 181522,1 & $-315559,5$ & $\mathrm{~B}$ \\
\hline $000.9-02.0$ & $\mathrm{Bl} 3-13$ & $000-021$ & 246,0 & & & 175251,7 & $-291051,9$ & $\mathrm{~B}$ \\
\hline $000.9-04.8$ & M $3-23$ & $000-042$ & $-153,0$ & 2,6 & 4,3 & 180352,9 & $-303441,0$ & B \\
\hline $001.0-02.6$ & Sa 3-104 & $001-022$ & 87,0 & & & 175514,5 & $-292035,0$ & $\mathrm{~B}$ \\
\hline $001.1-06.4$ & SB4 & $001-063$ & 21,0 & & & 181100,0 & $-311204,3$ & B \\
\hline $001.2+02.1$ & He $2-262$ & $001+021$ & $-172,4$ & 1,5 & 9,5 & 173705,6 & $-264248,7$ & $\mathrm{~B}$ \\
\hline $001.2-03.0$ & H $1-47$ & $001-031$ & 107,5 & & & 175726,2 & $-292146,3$ & $\mathrm{~B}$ \\
\hline $001.2-03.9$ & ShWi $2-5$ & $358-038$ & $-129,6$ & & & 180041,5 & $-295131,9$ & B \\
\hline $001.3-05.6$ & SB5 & $001-051$ & $-31,0$ & & & 180802,1 & $-303831,9$ & $\mathrm{~B}$ \\
\hline $001.4+05.3$ & H $1-15$ & $001+052$ & 33,0 & 5,6 & 9,1 & 172533,2 & $-244843,8$ & $\mathrm{~B}$ \\
\hline $001.4-03.4$ & ShWi 1 & $001-033$ & $-39,4$ & & & 175914,4 & $-292509,0$ & B \\
\hline $001.5-06.7$ & SwSt 1 & $001-062$ & $-17,0$ & 2,1 & & 181258,7 & $-305311,1$ & $\mathrm{~A}, \mathrm{~B}$ \\
\hline $001.6-05.9$ & SB6 & $001-052$ & 31,0 & & & 181002,8 & $-302649,4$ & $\mathrm{~B}$ \\
\hline $001.7+05.7$ & H $1-14$ & $001+051$ & 60,3 & 6,6 & 6,4 & 172458,0 & $-242257,4$ & B \\
\hline $001.7-01.6$ & $\mathrm{H} 2-31$ & $001-013$ & 120,0 & & & 175252,7 & $-281347,1$ & $\mathrm{~B}$ \\
\hline $001.7-04.4$ & H $1-55$ & $001-041$ & $-35,6$ & & & 180402,7 & $-294149,2$ & $\mathrm{~B}$ \\
\hline $001.7-04.6$ & H $1-56$ & $001-042$ & $-116,6$ & 3,6 & & 180441,9 & $-294501,8$ & $\mathrm{~A}, \mathrm{~B}$ \\
\hline $001.8-03.8$ & ShWi 7 & $358-0310$ & $-222,0$ & & & 180154,3 & $-292027,7$ & B \\
\hline $002.0-02.0$ & H $1-45$ & $002-021$ & $-19,2$ & & & 175512,2 & $-281438,2$ & $\mathrm{~B}$ \\
\hline $002.0-06.2$ & M $2-33$ & $002-061$ & $-126,0$ & & 8,7 & 181153,8 & $-3016 \quad 32,3$ & B \\
\hline $002.0-13.4$ & IC 4776 & $002-131$ & 16,3 & 3,3 & 5,0 & 184233,6 & $-332344,0$ & $\mathrm{~A}, \mathrm{~B}$ \\
\hline $002.1-02.2$ & M $3-20$ & $002-022$ & 24,3 & 3,7 & 7,7 & 175609,7 & $-281338,3$ & B \\
\hline $002.1-04.2$ & $\mathrm{H} 1-54$ & $002-041$ & $-137,4$ & & 12,3 & 180356,1 & $-291330,5$ & $\mathrm{~A}, \mathrm{~B}$ \\
\hline $002.2-02.5$ & KFL 2 & $002-02$ & $-80,3$ & & & 175750,2 & $-281617,2$ & B \\
\hline $002.2-02.7$ & M $2-23$ & $002-024$ & 186,7 & & 8,7 & 175832,7 & $-282544,8$ & $\mathrm{~A}, \mathrm{~B}$ \\
\hline $002.2-06.3$ & H $1-63$ & $002-062$ & $-29,4$ & 5,7 & & 181306,9 & $-300840,4$ & B \\
\hline $002.2-09.4$ & Cn $1-5$ & 002-09 1 & $-30,9$ & & 5,4 & 182557,2 & $-313159,1$ & $\mathrm{~B}$ \\
\hline $002.3-03.4$ & H $2-37$ & 002-032 & $-156,8$ & 2,0 & & 180118,6 & $-283747,2$ & $\mathrm{~A}, \mathrm{~B}$ \\
\hline $002.3-07.8$ & M 2-41 & $002-071$ & $-26,2$ & 3,9 & & 181921,0 & $-304501,2$ & B \\
\hline $002.4+05.8$ & NGC 6369 & $002+051$ & $-100,9$ & 0,6 & 1,1 & 172617,6 & $-234314,5$ & $\mathrm{~A}, \mathrm{~B}$ \\
\hline $002.4-03.7$ & M $1-38$ & $002-035$ & $-82,8$ & & & 180255,4 & $-284049,0$ & $\mathrm{~A}, \mathrm{~B}$ \\
\hline $002.5-01.7$ & Pe $2-11$ & $002-011$ & 155,0 & 1,3 & & 175522,5 & $-273652,4$ & $\mathrm{~B}$ \\
\hline $002.5-05.4$ & KFL 14 & $002-05$ & 93,0 & & & 180949,7 & $-292605,5$ & B \\
\hline $002.6+02.1$ & $\mathrm{~K} 5-13$ & $002+022$ & 237,2 & & & 174033,7 & $-253524,3$ & B \\
\hline $002.6+04.2$ & Th $3-27$ & $002+041$ & $-135,3$ & & & 173254,5 & $-242337,6$ & $\mathrm{~B}$ \\
\hline $002.6+05.5$ & $\mathrm{~K} 5-3$ & $002+052$ & 144,9 & & & 172738,3 & $-234245,9$ & B \\
\hline $002.6+08.1$ & H $1-11$ & $002+081$ & 4,3 & 6,7 & 7,5 & 171817,0 & $-221540,1$ & B \\
\hline
\end{tabular}

Continua na próxima página... 
Tabela A.1 - Continuação

\begin{tabular}{|c|c|c|c|c|c|c|c|c|}
\hline PN G & Nome & PK & $V_{r}($ hel $)$ & $d_{\mathrm{M} 84}$ & $d_{\mathrm{SSV} 08}$ & $\alpha(1950)$ & $\delta(1950)$ & Amostra \\
\hline $002.6-03.4$ & M 1-37 & $002-033$ & 220,5 & & & 180215,9 & $-2822 \quad 21,1$ & $\mathrm{~B}$ \\
\hline $002.7-04.8$ & M $1-42$ & $002-042$ & $-86,5$ & 4,3 & 5,5 & 180754,3 & $-285940,8$ & $\mathrm{~A}, \mathrm{~B}$ \\
\hline $002.7-52.4$ & IC $5148-50$ & $002-521$ & $-26,2$ & & 1,1 & 215633,5 & $-393732,0$ & B \\
\hline $002.8+01.7$ & H $2-20$ & $002+011$ & 164,1 & 1,0 & 9,4 & 174234,0 & $-253850,6$ & B \\
\hline $002.8+01.8$ & K $5-16$ & $002+012$ & $-32,6$ & & & 174222,6 & $-253700,2$ & $\mathrm{~B}$ \\
\hline $002.9+06.5$ & PM 1-149 & $002+061$ & 70,0 & & & 172451,4 & $-225455,1$ & B \\
\hline $002.9-03.9$ & H $2-39$ & $002-036$ & 36,6 & & & 180455,8 & $-282639,0$ & B \\
\hline $003.0-02.6$ & KFL 4 & $003-02$ & 17,0 & & & 175942,8 & $-274105,2$ & B \\
\hline $003.1+02.9$ & $\mathrm{Hb} 4$ & $003+021$ & $-60,0$ & 2,2 & 5,0 & 173848,4 & $-244042,2$ & $\mathrm{~A}, \mathrm{~B}$ \\
\hline $003.1+03.4$ & H $2-17$ & $003+031$ & 85,4 & 7,2 & 10,7 & 173703,4 & $-242409,1$ & B \\
\hline $003.2-04.4$ & KFL 12 & $003-04$ & 100,5 & & & 180721,0 & $-282002,0$ & B \\
\hline $003.2-06.2$ & M $2-36$ & $003-061$ & 73,0 & 4,0 & 6,1 & 181430,5 & $-290930,3$ & B \\
\hline $003.3-04.6$ & Ap $1-12$ & $003-047$ & 151,1 & 2,2 & 4,6 & 180825,3 & $-282320,3$ & B \\
\hline $003.3-06.1$ & SB7 & $003-062$ & $-27,0$ & & & 181435,5 & $-2907 \quad 18,2$ & B \\
\hline $003.3-07.5$ & KFL 19 & $003-07$ & 47,0 & & & 181957,1 & $-294459,3$ & B \\
\hline $003.4-04.8$ & H $2-43$ & $003-049$ & $-15,6$ & 2,6 & 5,1 & 180938,2 & $-282048,7$ & B \\
\hline $003.5-02.4$ & IC 4673 & $003-023$ & $-15,3$ & 3,4 & 3,2 & $1800 \quad 10,4$ & $-270630,2$ & $\mathrm{~A}, \mathrm{~B}$ \\
\hline $003.5-04.6$ & NGC 6565 & $003-045$ & $-5,9$ & 3,5 & 4,7 & 180842,9 & $-281127,3$ & $\mathrm{~A}, \mathrm{~B}$ \\
\hline $003.6+03.1$ & M 2-14 & $003+032$ & $-48,2$ & & & 173853,6 & $-240950,6$ & B \\
\hline $003.6+04.9$ & PM 1-159 & $003+041$ & $-85,5$ & & & 173229,0 & $-230954,2$ & B \\
\hline $003.6-02.3$ & M 2-26 & $003-022$ & $-52,9$ & 1,3 & 8,3 & 180004,0 & $-265837,4$ & B \\
\hline $003.7+07.9$ & $\mathrm{H} 2-8$ & $003+071$ & 26,3 & 25,2 & & 172146,0 & $-213055,8$ & B \\
\hline $003.7-04.6$ & M 2-30 & $003-048$ & 154,9 & & 9,4 & 180925,2 & $-275859,7$ & B \\
\hline $003.8+05.3$ & H $2-15$ & $003+051$ & $-63,2$ & 7,5 & 15,4 & 173125,0 & $-225121,6$ & B \\
\hline $003.8-04.3$ & H $1-59$ & $003-043$ & 7,0 & 2,6 & 9,4 & 180820,3 & $-274659,0$ & $\mathrm{~A}, \mathrm{~B}$ \\
\hline $003.8-04.5$ & $\mathrm{H} 2-41$ & $003-044$ & 26,3 & 2,5 & 8,6 & 180914,7 & $-275301,2$ & B \\
\hline $003.8-17.1$ & $\mathrm{Hb} 8$ & $003-171$ & 180,5 & 5,3 & 9,2 & 190220,1 & $-331613,4$ & B \\
\hline $003.9+02.6$ & IRAS $17414-2412$ & $003+022$ & 14,3 & & & 174129,3 & $-241215,0$ & B \\
\hline $003.9-02.3$ & M $1-35$ & $003-021$ & 67,9 & 0,7 & 6,1 & 180031,9 & $-264343,1$ & $\mathrm{~A}, \mathrm{~B}$ \\
\hline $003.9-03.1$ & KFL 7 & $003-03$ & $-91,0$ & & & 180342,0 & $-2706 \quad 39,1$ & $\mathrm{~B}$ \\
\hline $003.9-14.9$ & $\mathrm{Hb} 7$ & $003-141$ & $-65,3$ & 4,8 & 8,1 & 185223,1 & $-321941,1$ & $\mathrm{~B}$ \\
\hline $004.0-03.0$ & M 2-29 & $004-031$ & $-112,2$ & 1,2 & 8,6 & 180333,2 & $-265518,3$ & B \\
\hline $004.0-05.8$ & Pe $1-12$ & $004-053$ & 159,2 & 5,9 & 6,5 & 181432,7 & $-281824,3$ & $\mathrm{~B}$ \\
\hline $004.0-11.1$ & M 3-29 & $004-111$ & 49,3 & 3,0 & 5,8 & 183612,9 & $-304321,4$ & $\mathrm{~A}, \mathrm{~B}$ \\
\hline $004.1-03.8$ & KFL 11 & 004-03 B & $-39,2$ & & & 180704,0 & $-27 \quad 17 \quad 12,8$ & B \\
\hline $004.2-03.2$ & KFL 10 & 004-03 A & $-128,2$ & & & 180453,6 & $-265429,8$ & B \\
\hline $004.2-04.3$ & H $1-60$ & $004-041$ & 24,4 & 4,0 & 10,2 & 180916,7 & $-273000,2$ & B \\
\hline $004.2-05.2$ & SB8 & $004-056$ & 40,0 & & & 181241,3 & $-275002,0$ & B \\
\hline $004.2-05.9$ & M $2-37$ & $004-055$ & 59,1 & 3,8 & & 181528,9 & $-280915,5$ & B \\
\hline $004.3+01.8$ & H $2-24$ & $004+011$ & $-198,2$ & 6,3 & 8,8 & 174532,7 & $-241538,3$ & $\mathrm{~B}$ \\
\hline $004.3-02.6$ & H $1-53$ & $004-021$ & 75,0 & & & 180250,3 & $-263001,2$ & B \\
\hline $004.5+06.8$ & H $2-12$ & $004+061$ & $-176,8$ & 12,9 & & 172736,2 & $-212641,5$ & B \\
\hline $004.6+06.0$ & H $1-24$ & $004+062$ & 158,1 & 3,5 & 5,9 & 173037,3 & $-214423,4$ & B \\
\hline $004.6-09.9$ & SB9 & 004-09 1 & $-22,0$ & & & 183230,8 & $-294053,2$ & B \\
\hline $004.7-05.5$ & SB10 & $004-057$ & 54,0 & & & 181458,4 & $-273247,0$ & $\mathrm{~B}$ \\
\hline
\end{tabular}

Continua na próxima página. . 
Apêndice A. Amostra de Nebulosas Planetárias

Tabela A.1 - Continuação

\begin{tabular}{|c|c|c|c|c|c|c|c|c|}
\hline PN G & Nome & $\mathrm{PK}$ & $V_{r}($ hel $)$ & $d_{\mathrm{M} 84}$ & $d_{\mathrm{SSV} 08}$ & $\alpha(1950)$ & $\delta(1950)$ & Amostra \\
\hline $004.7-11.8$ & He $2-418$ & $004-112$ & 109,3 & 5,8 & & 184102,4 & $-302242,2$ & B \\
\hline $004.8+02.0$ & H $2-25$ & $004+021$ & 16,2 & 6,3 & 11,5 & 174557,4 & $-234159,9$ & B \\
\hline $004.8-05.0$ & M 3-26 & $004-051$ & $-9,8$ & 3,1 & 7,6 & 181303,3 & $-271601,4$ & B \\
\hline $004.9+04.9$ & M 1-25 & $004+041$ & 14,1 & 3,6 & 6,6 & 173529,5 & $-220658,4$ & $\mathrm{~A}, \mathrm{~B}$ \\
\hline $004.9-04.9$ & M 1-44 & $004-042$ & $-107,7$ & 1,8 & 10,3 & 181309,5 & $-270535,0$ & $\mathrm{~B}$ \\
\hline $005.0+03.0$ & Pe $1-9$ & $005+031$ & $-129,2$ & 3,5 & & 174234,8 & $-230117,1$ & B \\
\hline $005.0+04.4$ & $\mathrm{H} 1-27$ & $005+041$ & 19,0 & 4,7 & 7,7 & 173716,9 & $-221745,4$ & B \\
\hline $005.0-03.9$ & H $2-42$ & $005-032$ & $-118,7$ & 3,1 & & 180916,1 & $-263341,4$ & B \\
\hline $005.1+02.0$ & K 5-19 & $005+022$ & $-79,6$ & & & 174648,5 & $-232653,3$ & $\mathrm{~B}$ \\
\hline $005.1-03.0$ & H $1-58$ & $005-031$ & 74,0 & & & 180607,4 & $-260302,4$ & $\mathrm{~B}$ \\
\hline $005.1-08.9$ & Hf $2-2$ & $005-081$ & 54,9 & 4,5 & & 182920,8 & $-284535,3$ & B \\
\hline $005.2+04.2$ & M 3-13 & $005+042$ & 127,2 & & & 173835,7 & $-221136,1$ & $\mathrm{~B}$ \\
\hline $005.2+05.6$ & M 3-12 & $005+051$ & 16,5 & 3,1 & 7,8 & 173322,7 & $-212922,6$ & B \\
\hline $005.2-05.9$ & SB11 & $005-053$ & 95,0 & & & 181736,6 & $-271712,0$ & $\mathrm{~B}$ \\
\hline $005.2-18.6$ & StWr $2-21$ & $005-181$ & 194,4 & & & 191108,9 & $-323930,0$ & $\mathrm{~B}$ \\
\hline $005.4-06.1$ & SB12 & $005-062$ & 25,0 & & & 181847,1 & $-271115,0$ & B \\
\hline $005.5+02.7$ & H $1-34$ & $005+021$ & 13,3 & & & 174505,8 & $-224548,8$ & $\mathrm{~B}$ \\
\hline $005.5+06.1$ & M 3-11 & $005+061$ & $-2,1$ & 3,1 & 7,0 & 173222,3 & $-205529,5$ & $\mathrm{~B}$ \\
\hline $005.5-02.5$ & M 3-24 & $005-021$ & 119,2 & 1,1 & & 180448,4 & $-252430,5$ & B \\
\hline $005.5-04.0$ & H $2-44$ & $005-041$ & $-56,9$ & 2,7 & & 181033,9 & $-260931,3$ & $\mathrm{~B}$ \\
\hline $005.6-04.7$ & KFL 16 & $005-04$ & 35,0 & & & 181347,1 & $-262431,3$ & $\mathrm{~B}$ \\
\hline $005.7-03.6$ & KFL 13 & $005-03$ & $-81,7$ & & & 180939,1 & $-254513,4$ & B \\
\hline $005.7-05.3$ & M 2-38 & $005-051$ & $-77,3$ & 3,7 & 7,0 & 181618,0 & $-263638,0$ & $\mathrm{~B}$ \\
\hline $005.8+05.1$ & H $2-16$ & $005+052$ & $-56,6$ & 5,4 & & 173656,0 & $-211239,1$ & $\mathrm{~B}$ \\
\hline $005.8-06.1$ & NGC 6620 & $005-061$ & 69,6 & 4,0 & 8,8 & 181946,7 & $-265050,3$ & $\mathrm{~A}, \mathrm{~B}$ \\
\hline $006.0+03.1$ & M 1-28 & $006+032$ & 18,0 & 3,5 & 4,0 & 174437,9 & $-220525,0$ & B \\
\hline $006.0-03.6$ & M 2-31 & $006-033$ & 151,6 & & & 181010,4 & $-253056,5$ & B \\
\hline $006.1+08.3$ & M $1-20$ & $006+081$ & 59,9 & & & 172600,7 & $-191331,5$ & B \\
\hline $006.2-03.7$ & KFL 15 & $006-03$ & 127,8 & & & 181113,9 & $-252147,1$ & B \\
\hline $006.3+03.3$ & H $2-22$ & $006+031$ & $-3,0$ & 2,5 & & 174433,5 & $-214622,2$ & B \\
\hline $006.3+04.4$ & H $2-18$ & $006+041$ & $-113,4$ & 3,9 & & 174029,2 & $-210832,6$ & $\mathrm{~A}, \mathrm{~B}$ \\
\hline $006.4+02.0$ & M $1-31$ & $006+025$ & 68,8 & & 5,1 & 174940,2 & $-222118,4$ & B \\
\hline $006.4-04.6$ & Pe $2-13$ & $006-041$ & 32,4 & 6,0 & & 181507,6 & $-253923,3$ & B \\
\hline $006.5-03.1$ & H $1-61$ & $006-031$ & 52,5 & & & 180929,3 & $-245048,7$ & B \\
\hline $006.5-05.8$ & SB13 & $006-051$ & 57,0 & & & 181954,3 & $-260650,7$ & $\mathrm{~B}$ \\
\hline $006.7-02.2$ & M 1-41 & $006-021$ & $-5,9$ & 1,4 & 0,8 & 180626,1 & $-241258,3$ & B \\
\hline $006.8+02.0$ & Pe $2-10$ & $006+024$ & $-39,0$ & 1,0 & & 175036,5 & $-215807,3$ & B \\
\hline $006.8+02.3$ & $\mathrm{Th} 4-7$ & $006+023$ & 12,0 & & & 174922,0 & $-215033,5$ & $\mathrm{~B}$ \\
\hline $006.8+04.1$ & M $3-15$ & $006+042$ & 97,2 & 1,5 & & 174232,4 & $-205652,0$ & $\mathrm{~A}, \mathrm{~B}$ \\
\hline $006.8-03.4$ & $\mathrm{H} 2-45$ & $006-032$ & 91,1 & 2,3 & & 181124,3 & $-244434,9$ & B \\
\hline $006.8-08.6$ & Wray15-1876 & $006-081$ & 71,3 & & & 183147,6 & $-270844,9$ & $\mathrm{~B}$ \\
\hline $007.0+06.3$ & M $1-24$ & $007+062$ & $-26,3$ & 3,8 & & 173514,1 & $-193556,0$ & $\mathrm{~B}$ \\
\hline $007.0-06.0$ & H $1-66$ & $007-061$ & 85,1 & 4,0 & 8,3 & 182151,8 & $-254338,0$ & $\mathrm{~B}$ \\
\hline $007.0-06.8$ & Vy $2-1$ & $007-062$ & 107,7 & 3,9 & 8,0 & 182453,3 & $-260843,7$ & $\mathrm{~B}$ \\
\hline $007.2+01.8$ & $\mathrm{Hb} 6$ & $007+011$ & 10,3 & 1,9 & & 175206,6 & $-214412,1$ & $\mathrm{~A}, \mathrm{~B}$ \\
\hline
\end{tabular}

Continua na próxima página... 
Tabela A.1 - Continuação

\begin{tabular}{|c|c|c|c|c|c|c|c|c|}
\hline $\mathrm{PN} \mathrm{G}$ & Nome & PK & $V_{r}($ hel $)$ & $d_{\mathrm{M} 84}$ & $d_{\mathrm{SSV} 08}$ & $\alpha(1950)$ & $\delta(1950)$ & Amostra \\
\hline $007.5+04.3$ & Th 4-1 & $007+041$ & $-110,6$ & & & 174322,5 & $-201242,1$ & B \\
\hline $007.5+07.4$ & M $1-22$ & $007+071$ & $-1,4$ & 4,4 & 9,8 & 173214,2 & $-183225,7$ & B \\
\hline $007.6+06.9$ & M 1-23 & $007+061$ & $-73,5$ & 3,7 & & 173425,6 & $-184456,6$ & B \\
\hline $007.7-05.3$ & SB14 & $007-051$ & 31,0 & & & 182038,6 & $-244905,7$ & B \\
\hline $007.8-03.7$ & M $2-34$ & $007-031$ & 66,7 & 2,0 & & $1814 \quad 12,4$ & $-240002,5$ & B \\
\hline $007.8-04.4$ & H $1-65$ & $007-041$ & 122,4 & & 7,8 & 181705,0 & $-241626,3$ & $\mathrm{~B}$ \\
\hline $007.9+10.1$ & $\mathrm{MaC} 1-4$ & $007+101$ & $-45,0$ & & & 172344,4 & $-164558,4$ & B \\
\hline $008.0+03.9$ & NGC 6445 & $008+031$ & 16,2 & 1,0 & 1,4 & $1746 \quad 17,1$ & $-195941,0$ & $\mathrm{~A}, \mathrm{~B}$ \\
\hline $008.1-04.7$ & M 2-39 & $008-041$ & 72,7 & 8,8 & 12,1 & 181857,5 & $-24 \quad 1209,7$ & B \\
\hline $008.2+06.8$ & He $2-260$ & $008+061$ & 21,7 & & & 173601,4 & $-181557,0$ & $\mathrm{~B}$ \\
\hline $008.2-04.8$ & M $2-42$ & $008-042$ & 121,2 & 2,3 & 9,4 & 181928,4 & $-241100,1$ & $\mathrm{~B}$ \\
\hline $008.3-01.1$ & M 1-40 & $008-011$ & $-34,4$ & 1,9 & & 180524,8 & $-221723,5$ & $\mathrm{~A}, \mathrm{~B}$ \\
\hline $008.3-07.3$ & NGC 6644 & $008-072$ & 191,0 & 1,7 & & 182929,8 & $-250959,5$ & $\mathrm{~A}, \mathrm{~B}$ \\
\hline $008.4-03.6$ & H $1-64$ & $008-031$ & 82,6 & 2,1 & & 181521,3 & $-232610,9$ & B \\
\hline $008.6-02.6$ & $\mathrm{MaC} 1-11$ & $008-021$ & $-89,0$ & & & 181149,1 & $-224454,0$ & B \\
\hline $008.6-07.0$ & He $2-406$ & $008-071$ & 28,2 & & & 182848,5 & $-244828,9$ & $\mathrm{~B}$ \\
\hline $008.8+05.2$ & Th $4-2$ & $008+051$ & 44,8 & 2,5 & & 174313,5 & $-183824,0$ & B \\
\hline $009.0+04.1$ & Th $4-5$ & $009+041$ & $-19,4$ & 4,4 & 6,6 & 174731,9 & $-190222,0$ & B \\
\hline $009.3+02.8$ & Th 4-9 & $009+021$ & 64,0 & & & 175303,2 & $-192902,8$ & $\mathrm{~B}$ \\
\hline $009.3+04.1$ & Th 4-6 & $009+042$ & 16,0 & & & 174800,8 & $-184602,4$ & B \\
\hline $009.3-06.5$ & SB15 & $009-062$ & 165,0 & & & 182811,4 & $-240014,7$ & B \\
\hline $009.4-05.0$ & NGC 6629 & $009-051$ & 14,6 & 1,6 & 2,4 & 182240,2 & $-231355,8$ & $\mathrm{~A}, \mathrm{~B}$ \\
\hline $009.4-05.6$ & SB16 & $009-052$ & 61,0 & & & 182518,7 & $-232722,6$ & B \\
\hline $009.4-09.8$ & M 3-32 & 009-09 1 & 58,4 & 4,6 & 6,6 & 184138,1 & $-252442,5$ & B \\
\hline $009.6+10.5$ & A 41 & $009+101$ & 29,7 & 5,4 & 4,6 & 172610,3 & $-151043,2$ & B \\
\hline $009.6+14.8$ & NGC 6309 & $009+141$ & $-47,3$ & 2,1 & 2,7 & 171115,6 & $-125110,2$ & $\mathrm{~A}, \mathrm{~B}$ \\
\hline $009.6-10.6$ & M 3-33 & $009-101$ & 179,3 & 5,6 & 8,4 & 184507,2 & $-253215,3$ & $\mathrm{~B}$ \\
\hline $009.8-04.6$ & H $1-67$ & 009-04 1 & $-8,3$ & 2,5 & 7,8 & 182203,5 & $-223635,6$ & B \\
\hline $009.8-07.5$ & GJJC 1 & $010-081$ & $-32,0$ & & & 183319,7 & $-235751,4$ & B \\
\hline $010.1+00.7$ & NGC 6537 & $010+001$ & $-16,9$ & 0,9 & & 180215,2 & $-195051,2$ & $\mathrm{~A}, \mathrm{~B}$ \\
\hline $010.4+04.5$ & M $2-17$ & $010+041$ & $-39,0$ & 2,7 & 6,7 & 174909,9 & $-173524,2$ & $\mathrm{~B}$ \\
\hline $010.6+03.2$ & Th $4-10$ & $010+031$ & 28,0 & & & 175411,0 & $-180624,4$ & B \\
\hline $010.7+07.4$ & $\mathrm{Sa} 2-230$ & $010+071$ & 113,8 & & & 173909,2 & $-155442,7$ & $\mathrm{~B}$ \\
\hline $010.7-06.4$ & IC 4732 & $010-061$ & $-145,8$ & 3,4 & 6,8 & 183053,2 & $\begin{array}{lll}-22 & 41 & 02,2\end{array}$ & $\mathrm{~A}, \mathrm{~B}$ \\
\hline $010.7-06.7$ & Pe $1-13$ & $010-062$ & $-86,6$ & 6,6 & 9,2 & 183150,1 & $-224543,7$ & B \\
\hline $010.8+18.0$ & M 2-9 & $010+182$ & 87,5 & 3,3 & 1,7 & 170252,6 & $-100433,2$ & $\mathrm{~A}, \mathrm{~B}$ \\
\hline $010.8-01.8$ & NGC 6578 & $010-011$ & 4,4 & 2,1 & 3,6 & 181317,9 & $-202807,3$ & $\mathrm{~A}, \mathrm{~B}$ \\
\hline $011.0+05.8$ & NGC 6439 & $011+051$ & $-96,7$ & 3,8 & 6,3 & 174526,4 & $-162747,5$ & $\mathrm{~A}, \mathrm{~B}$ \\
\hline $011.0+06.2$ & M $2-15$ & $011+061$ & $-15,9$ & 5,4 & 7,6 & 174401,2 & $-161621,3$ & B \\
\hline $011.0-05.1$ & M $1-47$ & $011-051$ & $-67,2$ & 2,8 & 7,7 & 182610,8 & $-214854,9$ & B \\
\hline $011.1+07.0$ & Sa $2-237$ & $011+071$ & $-86,4$ & & & 174149,7 & $-154358,3$ & B \\
\hline $011.1+11.5$ & M $2-13$ & $011+111$ & 59,1 & & 16,6 & 172544,6 & $-132357,5$ & B \\
\hline $011.1-07.9$ & SB17 & $011-071$ & 145,0 & & & 183718,3 & $-225718,3$ & B \\
\hline $011.3+02.8$ & Th $4-11$ & $011+021$ & $-62,0$ & & & 175713,7 & $-174037,8$ & $\mathrm{~B}$ \\
\hline $011.3-09.4$ & H $2-48$ & 011-09 1 & $-5,3$ & & & 184332,9 & $-233004,0$ & $\mathrm{~B}$ \\
\hline
\end{tabular}

Continua na próxima página. . 
Tabela A.1 - Continuação

\begin{tabular}{|c|c|c|c|c|c|c|c|c|}
\hline PN G & Nome & PK & $V_{r}($ hel $)$ & $d_{\mathrm{M} 84}$ & $d_{\mathrm{SSV} 08}$ & $\alpha(1950)$ & $\delta(1950)$ & Amostra \\
\hline $011.4-07.3$ & SB18 & $011-072$ & $-2,0$ & & & 183542,0 & $-222656,0$ & B \\
\hline $011.7+00.0$ & M $1-43$ & $011-001$ & $-19,0$ & 0,7 & & 180852,4 & $-184706,4$ & B \\
\hline $011.7-00.6$ & NGC 6567 & $011-002$ & 119,3 & 2,1 & 3,6 & 181048,3 & $-190527,9$ & $\mathrm{~A}, \mathrm{~B}$ \\
\hline $011.7-06.6$ & M 1-55 & $011-061$ & $-22,4$ & & & 183333,6 & $-215137,0$ & B \\
\hline $011.9+04.2$ & M $1-32$ & $011+041$ & $-90,6$ & 0,8 & 4,7 & 175326,6 & $-162842,3$ & B \\
\hline $012.2+04.9$ & PM 1-188 & $012+041$ & 41,9 & & & 175128,3 & $-155521,0$ & B \\
\hline $012.5-09.8$ & M 1-62 & $012-091$ & 45,7 & 4,4 & & 184725,1 & $-223755,2$ & B \\
\hline $012.6-02.7$ & M $1-45$ & $012-021$ & 139,8 & & & 182010,9 & $-191840,0$ & B \\
\hline $013.0-04.3$ & Pe $2-14$ & $013-041$ & 88,1 & 3,1 & & 182702,0 & $-194242,1$ & $\mathrm{~B}$ \\
\hline $013.1+04.1$ & M 1-33 & $013+041$ & $-56,8$ & 1,2 & 7,3 & 175606,5 & $-153204,1$ & $\mathrm{~B}$ \\
\hline $013.3+32.7$ & Sn 1 & $013+321$ & $-125,5$ & & 12,9 & 161830,4 & $-000906,3$ & $\mathrm{~A}, \mathrm{~B}$ \\
\hline $013.4-03.9$ & M 1-48 & 013-031 & 128,7 & 7,3 & 10,0 & 182633,3 & $-190746,8$ & $\mathrm{~B}$ \\
\hline $013.7-10.6$ & $\mathrm{Y}-\mathrm{C} 2-32$ & $013-101$ & 34,4 & & & 185230,8 & $-215334,0$ & B \\
\hline $013.8-07.9$ & PC 21 & 013-07 1 & 43,2 & 5,5 & & 184236,8 & $-203810,0$ & $\mathrm{~B}$ \\
\hline $014.0-05.5$ & $\mathrm{~V}-\mathrm{V} 3-5$ & $014-051$ & $-45,5$ & & & 183335,3 & $-192201,8$ & B \\
\hline $014.2-07.3$ & M 3-31 & $014-071$ & $-88,3$ & & & 184104,2 & $-195757,6$ & B \\
\hline $014.3-05.5$ & $\mathrm{~V}-\mathrm{V} 3-6$ & $014-052$ & $-29,0$ & & & 183414,5 & $-190457,5$ & $\mathrm{~B}$ \\
\hline $014.4-06.1$ & SB19 & $014-061$ & 72,0 & & & 183643,3 & $-191658,3$ & B \\
\hline $014.6-04.3$ & M $1-50$ & $014-041$ & 11,5 & 3,9 & 6,0 & 183025,2 & $-181856,1$ & $\mathrm{~A}, \mathrm{~B}$ \\
\hline $014.7-11.8$ & Sa We 4 & $014-111$ & 20,0 & & & 185916,9 & $-213114,0$ & $\mathrm{~B}$ \\
\hline $014.8-08.4$ & SB20 & $014-081$ & $-24,0$ & & & 184626,9 & $-195544,0$ & $\mathrm{~B}$ \\
\hline $014.9+06.4$ & $\mathrm{~K} 2-5$ & $014+061$ & $-10,9$ & 5,7 & 3,6 & 175137,2 & $-124805,5$ & B \\
\hline $015.4-04.5$ & M 1-53 & $015-041$ & 53,6 & 3,6 & 5,7 & 183253,5 & $-173838,4$ & $\mathrm{~B}$ \\
\hline $015.6-03.0$ & A 44 & $015-031$ & 44,0 & 1,8 & 2,1 & 182717,5 & $-164731,7$ & $\mathrm{~B}$ \\
\hline $015.9+03.3$ & M 1-39 & $015+031$ & 99,3 & & 6,4 & 180440,9 & $-132914,3$ & B \\
\hline $016.0+13.5$ & A 42 & $016+131$ & 10,0 & 5,1 & & 172845,6 & $-081659,9$ & B \\
\hline $016.0-04.3$ & M $1-54$ & $016-041$ & $-47,4$ & 3,2 & 3,8 & 183314,4 & $-170228,2$ & $\mathrm{~A}, \mathrm{~B}$ \\
\hline $016.0-07.6$ & SB21 & $016-071$ & 66,0 & & & 184515,3 & $-183300,9$ & B \\
\hline $016.1-04.7$ & M $1-56$ & $016-042$ & $-3,8$ & 4,5 & & 183452,2 & $-170825,0$ & B \\
\hline $016.4-01.9$ & M $1-46$ & $016-011$ & 29,3 & 2,4 & 3,6 & 182504,0 & $-153450,3$ & B \\
\hline $016.7-07.3$ & SB22 & $016-072$ & $-10,0$ & & & 184535,5 & $-1747 \quad 18,3$ & B \\
\hline $017.3-21.9$ & A 65 & $017-211$ & 13,0 & 1,5 & 1,7 & 194334,7 & $-231536,5$ & B \\
\hline $017.5-07.4$ & SB23 & $017-071$ & 52,0 & & & 184719,4 & $-170553,3$ & B \\
\hline $017.5-09.2$ & SB24 & 017-09 1 & 22,0 & & & 185421,8 & $-175450,8$ & B \\
\hline $017.6-10.2$ & A 51 & $017-101$ & 16,8 & 2,7 & 1,5 & 185806,3 & $-181631,9$ & $\mathrm{~B}$ \\
\hline $017.7-02.9$ & M $1-52$ & $017-021$ & 133,1 & 2,1 & 7,0 & 183107,1 & $-145448,5$ & B \\
\hline $017.9-04.8$ & M 3-30 & $017-041$ & 53,5 & 4,3 & 4,5 & 183822,8 & $-153636,9$ & B \\
\hline $018.0+20.1$ & $\mathrm{Na} 1$ & $018+201$ & 18,4 & & 5,5 & 171014,4 & $-031229,4$ & B \\
\hline $018.6-02.2$ & M 3-54 & $018-021$ & 156,2 & & 8,6 & 183013,8 & $-134637,9$ & B \\
\hline $018.9+03.6$ & M 4- 8 & $018+031$ & 28,3 & & & 180923,2 & $-104345,5$ & B \\
\hline $018.9+04.1$ & M 3-52 & $018+041$ & $-14,0$ & 4,2 & 6,6 & 180740,3 & $-102942,7$ & B \\
\hline $019.2-02.2$ & M 4-10 & 019-02 1 & 49,6 & & & 183124,5 & $-131447,8$ & B \\
\hline $019.4-05.3$ & M 1-61 & $019-051$ & 29,9 & & & 184304,4 & $-143051,3$ & B \\
\hline $019.4-13.6$ & DeHt 3 & $019-131$ & $-37,2$ & & & 191409,8 & $-180701,9$ & B \\
\hline $019.7+03.2$ & M 3-25 & $019+031$ & 162,8 & & & 181231,2 & $-101110,2$ & B \\
\hline
\end{tabular}

Continua na próxima página... 
Tabela A.1 - Continuação

\begin{tabular}{|c|c|c|c|c|c|c|c|c|}
\hline $\mathrm{PN} \mathrm{G}$ & Nome & PK & $V_{r}($ hel $)$ & $d_{\mathrm{M} 84}$ & $d_{\mathrm{SSV} 08}$ & $\alpha(1950)$ & $\delta(1950)$ & Amostra \\
\hline $019.7-04.5$ & M 1-60 & 019-04 1 & 93,1 & & 9,4 & 184048,3 & $-134752,5$ & B \\
\hline $019.8+05.6$ & CTS 1 & $019+051$ & 86,0 & & & 180415,5 & $-085557,4$ & B \\
\hline $019.9+00.9$ & M 3-53 & $019+001$ & 20,6 & 0,9 & & 182121,1 & $-110821,6$ & B \\
\hline $020.7-05.9$ & Sa $1-8$ & $020-051$ & 33,6 & & 8,1 & 184754,8 & $-133436,8$ & $\mathrm{~B}$ \\
\hline $020.9-01.1$ & M $1-51$ & $021-011$ & $-6,7$ & 1,2 & 2,3 & 183042,2 & $-110946,4$ & $\mathrm{~A}, \mathrm{~B}$ \\
\hline $021.1-05.9$ & M 1-63 & $021-051$ & 8,5 & 3,2 & 9,8 & 184841,8 & $-131414,2$ & B \\
\hline $021.2-03.9$ & We $1-7$ & $021-031$ & $-11,0$ & & & 184118,2 & $\begin{array}{lll}-12 & 16 & 02,9\end{array}$ & $\mathrm{~B}$ \\
\hline $021.7-00.6$ & M 3-55 & $021-002$ & 9,7 & 1,4 & & 183029,0 & $-101738,4$ & B \\
\hline $021.8-00.4$ & M $3-28$ & $021-001$ & 4,7 & 0,9 & 4,9 & 182955,7 & $-100806,5$ & B \\
\hline $022.0-03.1$ & $\mathrm{M} 1-58$ & $022-031$ & 57,2 & 3,4 & 5,3 & 184010,3 & $-110953,9$ & $\mathrm{~B}$ \\
\hline $022.1-02.4$ & M $1-57$ & $022-021$ & 79,9 & 3,1 & 4,4 & 183734,0 & $-104236,9$ & $\mathrm{~A}, \mathrm{~B}$ \\
\hline $022.5+04.8$ & MA 2 & $022+041$ & 24,0 & & & 181231,4 & $-065812,9$ & B \\
\hline $023.0+04.3$ & MA 3 & $023+041$ & 119,0 & & & 181507,7 & $-064933,4$ & $\mathrm{~B}$ \\
\hline $023.3-07.6$ & $\mathrm{MaC} 1-16$ & $023-071$ & 54,0 & & & 185834,3 & $-120239,3$ & B \\
\hline $023.9-02.3$ & M 1-59 & $023-021$ & 85,9 & 2,5 & 5,4 & 184035,9 & $-090751,6$ & B \\
\hline $024.1+03.8$ & M $2-40$ & $024+031$ & 89,1 & 1,6 & 6,9 & 181843,0 & $-060322,9$ & $\mathrm{~B}$ \\
\hline $024.2+05.9$ & M 4-9 & $024+051$ & $-35,9$ & 1,5 & 1,8 & 181138,8 & $-050018,8$ & B \\
\hline $024.2-05.2$ & M 4-11 & $024-051$ & 42,7 & & & 185132,3 & $-100903,9$ & B \\
\hline $024.3-03.3$ & $\mathrm{Pe} 1-17$ & $024-031$ & 1,0 & 3,2 & 8,0 & 184504,4 & $-091228,7$ & $\mathrm{~A}, \mathrm{~B}$ \\
\hline $024.8-02.7$ & M 2-46 & $024-021$ & 66,7 & 1,4 & 12,1 & 184351,0 & $-083118,6$ & B \\
\hline $025.0-11.6$ & A 60 & $025-111$ & $-10,0$ & 3,1 & 1,7 & 191630,3 & $-122011,0$ & B \\
\hline $025.3+40.8$ & IC 4593 & $025+401$ & 22,0 & 2,4 & 3,2 & 160923,6 & $+121157,4$ & $\mathrm{~A}, \mathrm{~B}$ \\
\hline $025.3-04.6$ & K 4-8 & $025-041$ & $-85,0$ & & & 185136,1 & $-085122,9$ & B \\
\hline $025.4-04.7$ & IC 1295 & $025-042$ & $-15,8$ & 0,7 & 1,0 & 185153,3 & $-085329,9$ & B \\
\hline $025.8-17.9$ & NGC 6818 & $025-171$ & $-14,1$ & 1,5 & 2,1 & 194109,0 & $-141627,2$ & $\mathrm{~A}, \mathrm{~B}$ \\
\hline $025.9-10.9$ & $\mathrm{Na} 2$ & $026-111$ & 97,8 & & & 191533,3 & $-111146,5$ & B \\
\hline $027.3-02.1$ & Pe1-18 & $027-021$ & & 1,5 & & 184605,8 & $-055933,7$ & $\mathrm{~A}$ \\
\hline $027.3-03.4$ & A 49 & $027-031$ & 28,0 & 2,1 & & 185047,1 & $-063232,9$ & B \\
\hline $027.4-03.5$ & Vy $1-4$ & $027-032$ & 54,8 & & 10,1 & 185120,7 & $-063008,5$ & B \\
\hline $027.6+04.2$ & M 2-43 & $027+041$ & 96,4 & & & 182403,1 & $-024448,0$ & B \\
\hline $027.6-09.6$ & IC 4846 & 027-09 1 & 151,0 & 3,3 & & 191344,3 & $-090759,1$ & $\mathrm{~A}, \mathrm{~B}$ \\
\hline $028.0+10.2$ & WeSb 3 & $028+101$ & 49,0 & & & 180327,4 & $+002217,8$ & B \\
\hline $028.2-04.0$ & Pe $1-20$ & $028-041$ & 65,0 & 3,4 & 6,1 & 185436,7 & $-060354,1$ & $\mathrm{~B}$ \\
\hline $028.5+01.6$ & M 2-44 & $028+011$ & 106,0 & 0,8 & 4,8 & 183459,6 & $-030835,1$ & B \\
\hline $028.5+05.1$ & $\mathrm{~K} 3-2$ & $028+051$ & 41,0 & & 10,0 & 182225,0 & $-013236,0$ & B \\
\hline $028.7-03.9$ & Pe $1-21$ & $028-031$ & $-20,0$ & 2,5 & 5,1 & 185509,6 & $-053144,3$ & $\mathrm{~B}$ \\
\hline $029.2+00.0$ & TDC 1 & $029-001$ & $-55,0$ & & & 184215,7 & $-032342,4$ & B \\
\hline $029.2-05.9$ & NGC 6751 & $029-051$ & $-38,9$ & 2,8 & 2,6 & 190315,0 & $-060411,5$ & $\mathrm{~A}, \mathrm{~B}$ \\
\hline $029.8-07.8$ & LSA 1 & 029-07 1 & 89,0 & & & 1911 14,8 & $-062404,4$ & B \\
\hline $031.0-10.8$ & M 3-34 & $031-101$ & 48,3 & 4,4 & 6,6 & 192420,9 & $-064110,9$ & B \\
\hline $032.1+07.0$ & PC 19 & $032+072$ & 20,0 & & 10,4 & 182213,6 & $+022745,5$ & B \\
\hline $032.7-02.0$ & M 1-66 & $032-021$ & 27,9 & & 8,8 & 185551,2 & $-010753,0$ & B \\
\hline $032.9-02.8$ & K $3-19$ & $032-022$ & 3,5 & & & 185901,3 & $-012328,6$ & B \\
\hline $033.1-06.3$ & NGC 6772 & $033-061$ & 0,0 & 1,4 & 1,3 & 191159,5 & $-024740,2$ & B \\
\hline $033.8-02.6$ & NGC 6741 & $033-021$ & 41,3 & 1,7 & 3,7 & 190002,8 & $-003121,8$ & $\mathrm{~A}, \mathrm{~B}$ \\
\hline
\end{tabular}

Continua na próxima página... 
Apêndice A. Amostra de Nebulosas Planetárias

Tabela A.1 - Continuação

\begin{tabular}{|c|c|c|c|c|c|c|c|c|}
\hline PN G & Nome & PK & $V_{r}($ hel $)$ & $d_{\mathrm{M} 84}$ & $d_{\mathrm{SSV} 08}$ & $\alpha(1950)$ & $\delta(1950)$ & Amostra \\
\hline $034.5-06.7$ & NGC 6778 & $034-061$ & 91,0 & 2,2 & 3,2 & 191549,4 & $-0141 \quad 18,4$ & $\mathrm{~A}, \mathrm{~B}$ \\
\hline $034.6+11.8$ & NGC 6572 & $034+111$ & $-10,1$ & 0,8 & 1,7 & 180940,6 & $+065025,6$ & A, B \\
\hline $035.9-01.1$ & Sh $2-71$ & $036-011$ & 24,7 & 1,0 & 1,0 & 185927,9 & $+020455,0$ & B \\
\hline $036.0+17.6$ & A 43 & $036+171$ & $-42,0$ & & 1,6 & 175111,0 & $+103757,6$ & B \\
\hline $036.1-57.1$ & NGC 7293 & $036-57$ & $-28,2$ & 0,2 & 0,2 & 222654,7 & $-210536,5$ & $\mathrm{~A}, \mathrm{~B}$ \\
\hline $037.7-34.5$ & NGC 7009 & $037-341$ & $-46,6$ & 0,9 & 1,3 & 210127,6 & $-113345,6$ & $\mathrm{~A}, \mathrm{~B}$ \\
\hline $037.8-06.3$ & NGC 6790 & $037-061$ & 40,4 & 1,5 & & 192024,8 & $+012456,9$ & $\mathrm{~A}, \mathrm{~B}$ \\
\hline $038.1-25.4$ & A 70 & $038-251$ & $-79,0$ & 3,5 & 2,4 & 202852,8 & $-071528,7$ & B \\
\hline $038.2+12.0$ & Cn $3-1$ & $038+121$ & $-11,2$ & 2,9 & 6,0 & 181512,3 & $+100752,2$ & $\mathrm{~A}, \mathrm{~B}$ \\
\hline $038.7-03.3$ & M 1-69 & $038-032$ & 7,0 & & & 191124,2 & $+033229,4$ & B \\
\hline $039.5-02.7$ & M 2-47 & $039-021$ & 22,9 & 2,9 & 4,8 & 191105,9 & $+043253,0$ & B \\
\hline $041.8-02.9$ & NGC 6781 & $041-021$ & 4,3 & 0,9 & 0,7 & 191601,5 & $+062647,8$ & $\mathrm{~A}, \mathrm{~B}$ \\
\hline $042.5-14.5$ & NGC 6852 & $042-141$ & $-11,0$ & 3,4 & 2,7 & 195807,1 & $+013521,2$ & B \\
\hline $042.9-06.9$ & NGC 6807 & $042-061$ & $-67,7$ & 5,5 & 12,6 & 193205,8 & $+053425,5$ & $\mathrm{~A}, \mathrm{~B}$ \\
\hline $043.0-03.0$ & M 4-14 & $043-031$ & 31,7 & 1,6 & 6,8 & 191835,3 & $+073110,4$ & B \\
\hline $043.1+03.8$ & M 1-65 & $043+031$ & 3,7 & 2,3 & 9,2 & 185412,3 & $+104809,8$ & B \\
\hline $043.1+37.7$ & NGC 6210 & $043+371$ & $-36,2$ & 1,3 & 2,3 & 164223,6 & $+235329,0$ & $\mathrm{~A}, \mathrm{~B}$ \\
\hline $043.3+11.6$ & M $3-27$ & $043+111$ & $-6,0$ & & & 182531,6 & $+142709,7$ & B \\
\hline $045.4-02.7$ & Vy $2-2$ & $045-021$ & $-71,2$ & & & 192159,3 & $+094800,8$ & $\mathrm{~A}, \mathrm{~B}$ \\
\hline $045.7-04.5$ & NGC 6804 & $045-041$ & $-12,0$ & 1,6 & 1,7 & 192911,3 & $+090706,3$ & $\mathrm{~A}, \mathrm{~B}$ \\
\hline $046.4-04.1$ & NGC 6803 & $046-041$ & 12,9 & 2,5 & 5,3 & 192853,6 & $+095657,8$ & $\mathrm{~A}, \mathrm{~B}$ \\
\hline $048.7+01.9$ & He $2-429$ & $048+011$ & $-0,8$ & 0,8 & 6,7 & 191121,6 & $+145407,3$ & B \\
\hline $049.3+88.1$ & H $4-1$ & $049+881$ & $-141,0$ & 12,7 & 18,1 & 125702,9 & $+275420,4$ & B \\
\hline $051.3+01.8$ & PM 1-295 & $051+011$ & 13,0 & & & 191704,4 & $+170613,6$ & $\mathrm{~B}$ \\
\hline $051.4+09.6$ & Hu $2-1$ & $051+091$ & 14,0 & 1,9 & & 184738,6 & $+204708,0$ & A, B \\
\hline $051.9-03.8$ & M 1-73 & $051-031$ & $-13,8$ & 1,9 & 6,6 & 193851,6 & $+144955,2$ & B \\
\hline $052.2-04.0$ & M 1-74 & $052-041$ & 32,4 & & & 194001,3 & $+150200,1$ & A, B \\
\hline $052.5-02.9$ & Me $1-1$ & $052-022$ & $-6,0$ & 3,8 & 6,8 & 193653,3 & $+154952,5$ & $\mathrm{~A}, \mathrm{~B}$ \\
\hline $053.3+24.0$ & Vy $1-2$ & $053+241$ & $-102,0$ & 4,7 & 9,0 & 175224,7 & $+280026,9$ & $\mathrm{~A}, \mathrm{~B}$ \\
\hline $054.1-12.1$ & NGC 6891 & $054-121$ & 42,3 & 2,1 & 3,6 & 201247,5 & $+123302,9$ & $\mathrm{~A}, \mathrm{~B}$ \\
\hline $055.5-00.5$ & M 1-71 & $055-001$ & 24,7 & & & 193415,0 & $+193539,0$ & B \\
\hline $057.2-08.9$ & NGC 6879 & $057-081$ & 9,0 & 3,7 & 7,7 & 200810,0 & $+164625,5$ & $\mathrm{~A}, \mathrm{~B}$ \\
\hline $058.3-10.9$ & IC 4997 & $058-101$ & $-66,2$ & 2,3 & & 201751,4 & $+163422,3$ & $\mathrm{~A}, \mathrm{~B}$ \\
\hline $059.7-18.7$ & A 72 & $059-181$ & $-58,6$ & 1,1 & 1,2 & 204740,1 & $+132216,2$ & B \\
\hline $060.1-07.7$ & NGC 6886 & $060-072$ & $-35,8$ & 2,8 & 4,4 & 201029,4 & +1950 18,2 & $\mathrm{A}, \mathrm{B}$ \\
\hline $060.3-07.3$ & He $1-5$ & $060-071$ & 38,9 & 2,8 & 3,1 & 200943,0 & $+201103,3$ & B \\
\hline $060.8-03.6$ & NGC 6853 & $060-031$ & $-41,8$ & 0,4 & 0,3 & 195726,8 & $+223500,2$ & $\mathrm{~A}, \mathrm{~B}$ \\
\hline $061.4-09.5$ & NGC 6905 & 061-09 1 & $-8,4$ & 1,8 & 1,8 & 202009,1 & $+195636,9$ & A, B \\
\hline $061.8+02.1$ & He $2-442$ & $061+021$ & 38,7 & & & 193739,8 & $+262234,7$ & B \\
\hline $062.4+09.5$ & NGC 6765 & $062+091$ & $-60,0$ & 4,1 & 2,3 & 190910,2 & $+302740,5$ & $\mathrm{~B}$ \\
\hline $063.1+13.9$ & NGC 6720 & $063+131$ & $-19,2$ & 0,7 & 0,9 & 185143,5 & $+325756,8$ & $\mathrm{~A}, \mathrm{~B}$ \\
\hline $064.6+48.2$ & NGC 6058 & $064+481$ & 2,7 & 4,1 & 3,5 & 160243,1 & $+404903,4$ & B \\
\hline $064.7+05.0$ & BD+30 3639 & $064+051$ & $-31,4$ & 0,6 & 3,0 & 193247,5 & $+302420,7$ & $\mathrm{~A}, \mathrm{~B}$ \\
\hline $064.9+15.5$ & M 1-64 & $064+151$ & $-25,1$ & 3,1 & 5,6 & 184814,4 & $+351102,8$ & B \\
\hline $065.0-27.3$ & Ps 1 & $065-271$ & $-140,6$ & 9,3 & 10,0 & 212734,4 & $+115715,6$ & B \\
\hline
\end{tabular}

Continua na próxima página... 
Tabela A.1 - Continuação

\begin{tabular}{|c|c|c|c|c|c|c|c|c|}
\hline PN G & Nome & PK & $V_{r}($ hel $)$ & $d_{\mathrm{M} 84}$ & $d_{\mathrm{SSV} 08}$ & $\alpha(1950)$ & $\delta(1950)$ & Amostra \\
\hline $065.2-05.6$ & He $1-6$ & $065-051$ & $-38,3$ & 6,9 & 3,1 & 201513,8 & $+251225,3$ & $\mathrm{~B}$ \\
\hline $065.9+00.5$ & NGC 6842 & $065+001$ & $-5,0$ & 1,7 & 1,4 & 195301,5 & $+290921,1$ & B \\
\hline $066.7-28.2$ & NGC 7094 & $066-281$ & $-101,1$ & 2,9 & 1,4 & 213428,0 & $+123349,0$ & B \\
\hline $068.3-02.7$ & He $2-459$ & $068-021$ & $-72,0$ & & & 201155,7 & $+292446,6$ & B \\
\hline $068.6+01.1$ & He $1-4$ & $068+012$ & $-10,5$ & 1,9 & & 195720,3 & $+314624,4$ & B \\
\hline $068.8+00.0$ & M $1-75$ & $068-001$ & $-25,3$ & 3,1 & 3,9 & 200245,3 & $+311849,2$ & $\mathrm{~A}, \mathrm{~B}$ \\
\hline $069.4-02.6$ & NGC 6894 & $069-021$ & $-58,0$ & 1,5 & 1,7 & 201422,9 & $+302435,0$ & $\mathrm{~A}, \mathrm{~B}$ \\
\hline $071.6-02.3$ & M 3-35 & $071-021$ & $-192,2$ & & & 201904,7 & $+321948,9$ & B \\
\hline $072.7-17.1$ & A 74 & $072-171$ & 18,0 & 0,6 & & 211438,1 & $+235616,1$ & B \\
\hline $074.5+02.1$ & NGC 6881 & $074+021$ & $-14,4$ & 1,7 & 5,3 & 200901,7 & $+371544,1$ & $\mathrm{~A}, \mathrm{~B}$ \\
\hline $077.6+14.7$ & A 61 & $077+141$ & $-48,0$ & 1,7 & & 191742,1 & $+460915,7$ & B \\
\hline $078.5+18.7$ & A 50 & $078+181$ & $-159,0$ & 2,8 & 5,1 & 185759,1 & $+482341,5$ & B \\
\hline $079.6+05.8$ & M $4-17$ & $079+051$ & $-26,0$ & 1,8 & & 200722,2 & $+433451,7$ & B \\
\hline $081.2-14.9$ & A 78 & $081-141$ & 17,0 & 4,0 & 1,6 & 213320,0 & $+312818,6$ & B \\
\hline $082.1+07.0$ & NGC 6884 & $082+071$ & $-35,6$ & 1,7 & 3,8 & 200849,1 & $+461842,8$ & $\mathrm{~A}, \mathrm{~B}$ \\
\hline $082.5+11.3$ & NGC 6833 & $082+111$ & $-108,8$ & 2,6 & & 194820,9 & $+485001,1$ & $\mathrm{~A}, \mathrm{~B}$ \\
\hline $083.5+12.7$ & NGC 6826 & $083+121$ & $-6,2$ & 0,7 & 1,6 & 194327,1 & $+502410,9$ & $\mathrm{~A}, \mathrm{~B}$ \\
\hline $084.9-03.4$ & NGC 7027 & $084-031$ & 8,8 & 0,7 & & 210509,4 & $+420203,8$ & $\mathrm{~A}, \mathrm{~B}$ \\
\hline $086.5-08.8$ & $\mathrm{Hu} 1-2$ & $086-081$ & 9,9 & 2,2 & & 213107,7 & $+392449,0$ & $\mathrm{~A}, \mathrm{~B}$ \\
\hline $088.7-01.6$ & NGC 7048 & $088-011$ & $-50,2$ & 1,2 & 1,6 & 211227,7 & $+460448,9$ & B \\
\hline $089.0+00.3$ & NGC 7026 & $089+001$ & $-40,6$ & 0,9 & 2,4 & 210435,2 & $+473901,9$ & $\mathrm{~A}, \mathrm{~B}$ \\
\hline $089.8-05.1$ & IC 5117 & $089-051$ & $-26,1$ & 1,7 & & 213036,8 & $+442228,3$ & $\mathrm{~A}, \mathrm{~B}$ \\
\hline $093.3-02.4$ & M 1-79 & 093-02 1 & $-24,0$ & 1,0 & 2,7 & 213512,6 & $+484231,6$ & $\mathrm{~A}, \mathrm{~B}$ \\
\hline $093.4+05.4$ & NGC 7008 & $093+052$ & $-75,7$ & 0,9 & 0,9 & 205904,9 & $+542048,2$ & $\mathrm{~A}, \mathrm{~B}$ \\
\hline $093.5+01.4$ & M $1-78$ & $093+011$ & $-88,1$ & 3,1 & & 211905,6 & $+514040,5$ & $\mathrm{~A}, \mathrm{~B}$ \\
\hline $095.1-02.0$ & M 2-49 & $095-021$ & $-134,0$ & & & 214129,3 & $+501127,6$ & B \\
\hline $095.2+00.7$ & K $3-62$ & $095+001$ & $-53,0$ & & & 213009,0 & $+522034,0$ & B \\
\hline $096.3+02.3$ & $\mathrm{~K} 3-61$ & $096+021$ & & 1,1 & 7,4 & 212823,9 & $+541414,2$ & $\mathrm{~A}$ \\
\hline $096.4+29.9$ & NGC 6543 & $096+291$ & $-66,1$ & 0,7 & 1,6 & 175834,2 & $+663805,4$ & $\mathrm{~A}, \mathrm{~B}$ \\
\hline $097.5+03.1$ & A 77 & $097+031$ & $-113,4$ & 1,5 & 0,9 & 213035,9 & $+553923,7$ & B \\
\hline $097.6-02.4$ & M 2-50 & 097-02 1 & $-136,0$ & 1,5 & 10,1 & 215551,1 & $+512718,6$ & A, B \\
\hline $100.0-08.7$ & Me $2-2$ & $100-081$ & $-152,0$ & & & 222937,8 & $+473237,1$ & $\mathrm{~A}, \mathrm{~B}$ \\
\hline $100.6-05.4$ & IC 5217 & $100-051$ & $-98,6$ & 2,8 & 5,4 & 222155,7 & $+504247,0$ & $\mathrm{~A}, \mathrm{~B}$ \\
\hline $103.2+00.6$ & M 2-51 & $103+001$ & $-11,0$ & 1,5 & 1,9 & 221416,2 & $+571334,7$ & $\mathrm{~B}$ \\
\hline $103.7+00.4$ & M $2-52$ & $103+002$ & $-92,0$ & 1,5 & 4,5 & 221841,1 & $+572112,2$ & B \\
\hline $104.1+07.9$ & NGC 7139 & $104+071$ & $-54,2$ & 1,6 & 1,4 & 214449,6 & $+633335,1$ & B \\
\hline $104.2-29.6$ & Jn 1 & $104-291$ & $-67,0$ & 0,9 & 0,7 & 233323,9 & $+301130,4$ & B \\
\hline $104.4-01.6$ & M $2-53$ & $104-011$ & $-62,0$ & 0,9 & 3,8 & 223021,4 & $+555458,2$ & B \\
\hline $106.5-17.6$ & NGC 7662 & $106-171$ & $-13,2$ & 0,8 & 2,0 & 232329,1 & $+421536,0$ & $\mathrm{~A}, \mathrm{~B}$ \\
\hline $107.6-13.3$ & Vy $2-3$ & $107-131$ & $-49,5$ & 7,4 & 11,8 & 232035,9 & $+463729,6$ & $\mathrm{~B}$ \\
\hline $107.7+07.8$ & IsWe 2 & $107+071$ & $-8,0$ & & & 221155,8 & $+653901,4$ & B \\
\hline $107.7-02.2$ & M 1-80 & $107-021$ & $-58,2$ & 1,6 & 5,5 & 225414,8 & $+565318,7$ & A, B \\
\hline $107.8+02.3$ & NGC 7354 & $107+021$ & $-41,8$ & 0,8 & 1,7 & 223827,8 & $+610128,0$ & $\mathrm{~A}, \mathrm{~B}$ \\
\hline $108.4-76.1$ & BoBn 1 & $108-761$ & 196,0 & & & 003444,9 & $-135928,6$ & B \\
\hline $110.1+01.9$ & PM 1-339 & $110+011$ & $-80,0$ & & & 225654,5 & $+614152,5$ & $\mathrm{~B}$ \\
\hline
\end{tabular}

Continua na próxima página... 
Tabela A.1 - Continuação

\begin{tabular}{|c|c|c|c|c|c|c|c|c|}
\hline $\mathrm{PN} G$ & Nome & PK & $V_{r}($ hel $)$ & $d_{\mathrm{M} 84}$ & $d_{\mathrm{SSV} 08}$ & $\alpha(1950)$ & $\delta(1950)$ & Amostra \\
\hline $111.2+07.0$ & $\mathrm{KjPn} 6$ & $111+061$ & $-68,3$ & & & 224716,6 & $+664545,5$ & $\mathrm{~B}$ \\
\hline $111.8-02.8$ & $\mathrm{Hb} 12$ & $111-021$ & $-5,0$ & 0,5 & & 232357,3 & $+575424,2$ & A, B \\
\hline $112.5+03.7$ & K 3-88 & $112+031$ & $-54,7$ & & & 231011,3 & $+642258,4$ & B \\
\hline $112.5-00.1$ & $\mathrm{Kj} \operatorname{Pn} 8$ & $112-001$ & $-40,6$ & & & 232155,9 & $+604101,7$ & B \\
\hline $114.0-04.6$ & A 82 & $114-041$ & $-30,5$ & 2,0 & 1,9 & 234320,9 & $+564718,8$ & $\mathrm{~B}$ \\
\hline $116.2+08.5$ & M 2-55 & $116+081$ & $-22,4$ & 1,9 & 2,2 & 232942,5 & $+700536,3$ & B \\
\hline $118.0-08.6$ & Vy $1-1$ & $118-081$ & $-50,4$ & & 6,3 & 001601,5 & $+533541,1$ & $\mathrm{~A}, \mathrm{~B}$ \\
\hline $118.8-74.7$ & NGC 246 & $118-741$ & $-46,0$ & 0,4 & 0,5 & 004432,5 & $-120841,1$ & $\mathrm{~A}, \mathrm{~B}$ \\
\hline $119.3+00.3$ & BV 5-1 & $119+001$ & $-71,9$ & & & 001716,1 & $+624224,5$ & $\mathrm{~B}$ \\
\hline $119.6-06.7$ & $\mathrm{Hu} 1-1$ & $119-061$ & $-53,7$ & 1,9 & 7,5 & 002530,1 & $+554119,4$ & A, B \\
\hline $120.0+09.8$ & NGC 40 & $120+091$ & $-20,4$ & 0,8 & 1,2 & 001016,7 & $+721438,2$ & $\mathrm{~A}, \mathrm{~B}$ \\
\hline $120.2-05.3$ & Sh $2-176$ & $120-051$ & $-37,0$ & & & 002906,0 & +5706 & $\mathrm{~B}$ \\
\hline $121.6+00.0$ & BV $5-2$ & $121+001$ & $-39,0$ & & & 003726,0 & $+623506,7$ & B \\
\hline $121.6+03.5$ & We $1-1$ & $121+031$ & $-77,3$ & & & 003555,7 & $+660720,0$ & $\mathrm{~B}$ \\
\hline $122.1-04.9$ & A 2 & $122-041$ & $-41,8$ & 3,0 & 4,0 & 004240,6 & $+574111,4$ & $\mathrm{~B}$ \\
\hline $123.6+34.5$ & IC 3568 & $123+341$ & $-41,1$ & 2,1 & 2,7 & 123146,7 & $+825022,0$ & $\mathrm{~A}, \mathrm{~B}$ \\
\hline $126.3+02.9$ & K $3-90$ & $126+031$ & $-49,4$ & & 5,8 & 012132,8 & $+652258,9$ & $\mathrm{~B}$ \\
\hline $128.0-04.1$ & Simeiz 22 & $128-041$ & $-26,0$ & & & 012718,4 & $+580923,2$ & $\mathrm{~B}$ \\
\hline $130.2+01.3$ & IC 1747 & $130+011$ & $-66,5$ & 0,9 & 3,0 & 015358,0 & $+630441,5$ & $\mathrm{~A}, \mathrm{~B}$ \\
\hline $130.3-11.7$ & M 1-1 & $130-111$ & $-38,0$ & 6,1 & 8,0 & 013413,0 & $+501255,8$ & $\mathrm{~A}, \mathrm{~B}$ \\
\hline $130.4+03.1$ & K $3-92$ & $130+031$ & $-61,7$ & & 6,9 & 015955,3 & $+644314,1$ & B \\
\hline $130.9-10.5$ & NGC650-51 & $130-101$ & $-19,1$ & 0,7 & 0,7 & 013910,5 & $+511924,4$ & $\mathrm{~A}, \mathrm{~B}$ \\
\hline $131.4-05.4$ & BV $5-3$ & $131-051$ & $-59,0$ & & & 014942,9 & $+560931,2$ & $\mathrm{~B}$ \\
\hline $133.1-08.6$ & M 1-2 & $133-081$ & $-12,1$ & & & 015532,9 & $+523914,6$ & $\mathrm{~B}$ \\
\hline $136.3+05.5$ & HFG 1 & $136+05$ & $-26,0$ & & & 025932,1 & $+644252,4$ & B \\
\hline $138.8+02.8$ & IC 289 & $138+021$ & $-13,4$ & 1,3 & 1,4 & 030616,5 & $+610738,6$ & B \\
\hline $144.5+06.5$ & NGC 1501 & $144+061$ & 36,2 & 1,1 & 1,2 & 040240,7 & $+604711,9$ & $\mathrm{~B}$ \\
\hline $146.7+07.6$ & M 4-18 & $146+071$ & $-17,0$ & & 9,1 & 042131,2 & $+600024,0$ & B \\
\hline $147.4-02.3$ & M 1-4 & $147-021$ & $-33,0$ & 0,8 & 6,6 & 033759,1 & $+520725,3$ & $\mathrm{~A}, \mathrm{~B}$ \\
\hline $147.8+04.1$ & M 2-2 & $147+041$ & $-7,0$ & 1,4 & 5,2 & 040910,2 & $+564920,2$ & $\mathrm{~A}, \mathrm{~B}$ \\
\hline $148.4+57.0$ & NGC 3587 & $148+571$ & 6,0 & 0,7 & 0,6 & 111154,6 & $+551729,8$ & $\mathrm{~A}, \mathrm{~B}$ \\
\hline $149.7-03.3$ & IsWe 1 & $150-03$ & $-1,5$ & & & 034525,7 & $+495106,4$ & B \\
\hline $158.8+37.1$ & A 28 & $158+371$ & $-2,0$ & 2,4 & 0,9 & 083738,8 & $+582431,3$ & B \\
\hline $159.0-15.1$ & IC 351 & $159-151$ & $-9,1$ & 3,0 & 5,6 & 034420,3 & $+345335,4$ & $\mathrm{~A}, \mathrm{~B}$ \\
\hline $161.2-14.8$ & IC 2003 & $161-141$ & $-15,5$ & 2,4 & 5,5 & 035310,1 & $+334348,6$ & $\mathrm{~A}, \mathrm{~B}$ \\
\hline $164.8+31.1$ & JnEr 1 & $164+311$ & $-84,3$ & 0,9 & & 075358,9 & $+533322,5$ & B \\
\hline $165.5-06.5$ & $\mathrm{~K} 3-67$ & $165-061$ & $-77,0$ & & 3,4 & 043627,5 & $+363952,2$ & $\mathrm{~A}, \mathrm{~B}$ \\
\hline $165.5-15.2$ & NGC 1514 & $165-151$ & 59,8 & 0,8 & 0,8 & 040608,3 & $+303841,8$ & $\mathrm{~A}, \mathrm{~B}$ \\
\hline $166.1+10.4$ & IC 2149 & $166+101$ & $-30,7$ & 0,7 & 3,3 & 055240,7 & $+460553,7$ & $\mathrm{~A}, \mathrm{~B}$ \\
\hline $167.0-00.9$ & A 8 & $167-001$ & 58,2 & 2,6 & 1,6 & 050311,9 & $+390410,1$ & $\mathrm{~B}$ \\
\hline $167.4-09.1$ & K $3-66$ & $167-091$ & $-67,5$ & & 12,9 & 043322,1 & $+333326,8$ & $\mathrm{~A}, \mathrm{~B}$ \\
\hline $169.6+00.0$ & IC 2120 & $169-001$ & $-8,0$ & & 1,4 & 051446,2 & $+373018,2$ & B \\
\hline $170.3+15.8$ & NGC 2242 & $170+15$ & & & & 063027,9 & $+444858,8$ & A \\
\hline $170.7+04.6$ & K $3-69$ & $170+041$ & 22,9 & & & 053754,0 & +3913 39,2 & B \\
\hline $171.3-25.8$ & $\mathrm{Ba} 1$ & $171-251$ & $-17,0$ & 3,6 & 2,5 & 035042,8 & $+192048,2$ & B \\
\hline
\end{tabular}

Continua na próxima página... 
Tabela A.1 - Continuação

\begin{tabular}{|c|c|c|c|c|c|c|c|c|}
\hline PN G & Nome & PK & $V_{r}($ hel $)$ & $d_{\mathrm{M} 84}$ & $d_{\mathrm{SSV} 08}$ & $\alpha(1950)$ & $\delta(1950)$ & Amostra \\
\hline $173.5+03.2$ & $\mathrm{Pu} 2$ & $173+031$ & 45,0 & & & 053912,1 & $+360744,3$ & B \\
\hline $173.7-05.8$ & K $2-1$ & $173-051$ & 19,2 & 1,4 & 1,1 & 050356,7 & $+304532,3$ & $\mathrm{~A}, \mathrm{~B}$ \\
\hline $174.2-14.6$ & H $3-29$ & $174-141$ & $-19,9$ & & 4,6 & 043420,7 & $+245641,3$ & $\mathrm{~A}, \mathrm{~B}$ \\
\hline $178.3-02.5$ & K $3-68$ & $178-021$ & 65,0 & & 6,0 & 052825,5 & $+285630,8$ & $\mathrm{~A}, \mathrm{~B}$ \\
\hline $181.5+00.9$ & $\mathrm{Pu} 1$ & $181+001$ & 38,6 & & 2,0 & 054939,0 & $+280520,4$ & B \\
\hline $184.0-02.1$ & M $1-5$ & $184-021$ & 37,0 & & & 054346,1 & $+242058,6$ & $\mathrm{~A}, \mathrm{~B}$ \\
\hline $184.6+00.6$ & K $3-70$ & $184+001$ & 26,9 & & 19,4 & 055540,0 & $+251831,6$ & B \\
\hline $184.8+04.4$ & K $3-71$ & $184+041$ & 18,0 & & & 061047,4 & $+265351,0$ & B \\
\hline $189.1+19.8$ & NGC 2371-72 & $189+191$ & 20,8 & 1,5 & 1,6 & 072225,6 & $+293525,5$ & $\mathrm{~A}, \mathrm{~B}$ \\
\hline $189.8+07.7$ & M 1-7 & $189+071$ & 2,1 & 5,7 & 6,0 & 063417,8 & $+240312,6$ & $\mathrm{~A}, \mathrm{~B}$ \\
\hline $190.3-17.7$ & J 320 & $190-171$ & $-23,4$ & 4,1 & 6,1 & 050248,4 & $+103821,0$ & $\mathrm{~A}, \mathrm{~B}$ \\
\hline $193.6-09.5$ & Н $3-75$ & 193-09 1 & 22,9 & & & 053756,7 & $+121952,3$ & $\mathrm{~A}, \mathrm{~B}$ \\
\hline $194.2+02.5$ & J 900 & $194+021$ & 47,2 & 2,1 & 4,9 & 062302,2 & $+174914,4$ & $\mathrm{~A}, \mathrm{~B}$ \\
\hline $196.6-10.9$ & NGC 2022 & $196-101$ & 14,0 & 2,2 & 2,5 & 053921,8 & $+090346,1$ & $\mathrm{~A}, \mathrm{~B}$ \\
\hline $197.2-14.2$ & K $1-7$ & $197-141$ & 57,6 & 3,0 & 3,9 & 052903,7 & $+065352,5$ & $\mathrm{~A}, \mathrm{~B}$ \\
\hline $197.4-06.4$ & WeDe 1 & $197-061$ & 16,0 & & & 055638,5 & $+104131,8$ & B \\
\hline $197.8+17.3$ & NGC 2392 & $197+171$ & 75,0 & 1,1 & 1,3 & 072613,3 & $+210056,6$ & $\mathrm{~A}, \mathrm{~B}$ \\
\hline $198.6-06.3$ & A 12 & $198-061$ & & 2,6 & 2,1 & 055934,9 & +09 3918,3 & A \\
\hline $201.7+02.5$ & $\mathrm{~K} 4-48$ & $201+021$ & & & 13,5 & 063709,1 & $+110918,1$ & A \\
\hline $204.0-08.5$ & A 13 & $204-081$ & & 1,8 & 1,2 & 060209,6 & $+035650,9$ & A \\
\hline $204.1+04.7$ & K $2-2$ & $204+041$ & 28,0 & 1,0 & 0,4 & 064938,0 & $+100136,9$ & B \\
\hline $205.1+14.2$ & A $21 /$ YM 29 & $205+141$ & 28,8 & 0,5 & 0,2 & 072614,3 & $+132102,4$ & $\mathrm{~A}, \mathrm{~B}$ \\
\hline $205.8-26.7$ & $\mathrm{MaC} 2-1$ & $205-261$ & 74,1 & & & 050115,1 & -0614 12,0 & $\mathrm{A}, \mathrm{B}$ \\
\hline $206.4-40.5$ & NGC 1535 & $206-401$ & $-3,2$ & 1,6 & 2,3 & 041155,6 & $-125152,5$ & $\mathrm{~A}, \mathrm{~B}$ \\
\hline $210.3+01.9$ & M 1-8 & $210+011$ & 52,0 & 3,5 & 3,4 & 065056,5 & $+031213,5$ & $\mathrm{~A}, \mathrm{~B}$ \\
\hline $211.2-03.5$ & M 1-6 & $211-031$ & 65,6 & 3,0 & & 063311,5 & $-000306,9$ & $\mathrm{~A}, \mathrm{~B}$ \\
\hline $212.0+04.3$ & M 1-9 & $212+041$ & 118,0 & & 11,6 & 070242,3 & $+025135,8$ & $\mathrm{~A}, \mathrm{~B}$ \\
\hline $214.9+07.8$ & A 20 & $214+071$ & & 3,0 & 2,0 & 072022,0 & $+015122,4$ & A \\
\hline $215.2-24.2$ & IC 418 & $215-241$ & 61,9 & 0,8 & & 052509,4 & $-1244 \quad 17,2$ & $\mathrm{~A}, \mathrm{~B}$ \\
\hline $215.5-30.8$ & A 7 & $215-301$ & 18,0 & 0,5 & 0,2 & 050051,9 & $-154033,7$ & B \\
\hline $215.6+03.6$ & NGC 2346 & $215+031$ & 21,8 & 1,5 & 1,4 & 070649,6 & $-004330,2$ & A, B \\
\hline $215.6+11.1$ & K $1-11$ & $215+111$ & 26,8 & 2,1 & & 073331,2 & $+024910,6$ & B \\
\hline $216.0-00.2$ & A 18 & $216-001$ & & 1,7 & 1,6 & 065344,2 & $-024909,7$ & A \\
\hline $217.0+00.0$ & $\mathrm{MaC} 1-1$ & $217-001$ & 53,0 & & & 065614,6 & $-033659,8$ & B \\
\hline $217.1+14.7$ & A 24 & $217+141$ & 12,7 & 0,9 & 0,5 & 074900,6 & $+030805,0$ & $\mathrm{~A}, \mathrm{~B}$ \\
\hline $217.4+02.0$ & St $3-1$ & $217+021$ & 54,5 & & & 070420,6 & $-030026,6$ & B \\
\hline $219.1+31.2$ & A 31 & $219+311$ & 41,0 & 0,5 & 0,3 & 085131,7 & +0905 19,4 & B \\
\hline $220.3-53.9$ & NGC 1360 & $220-531$ & 41,8 & 0,5 & 0,4 & 033106,9 & $-2602 \quad 21,9$ & B \\
\hline $221.3-12.3$ & IC 2165 & $221-121$ & 53,9 & 1,9 & 3,7 & 061924,4 & $-125744,2$ & $\mathrm{~A}, \mathrm{~B}$ \\
\hline $221.7+05.3$ & M 3-3 & $221+051$ & 95,0 & 2,5 & 5,8 & 072406,3 & $-051547,5$ & $\mathrm{~A}, \mathrm{~B}$ \\
\hline $224.3+15.3$ & K $1-13$ & $224+151$ & 16,0 & 2,1 & 0,8 & 080415,6 & $-024352,9$ & B \\
\hline $226.7+05.6$ & M 1-16 & $226+051$ & 65,6 & 5,5 & 10,0 & 073455,5 & $-093159,8$ & $\mathrm{~A}, \mathrm{~B}$ \\
\hline $228.8+05.3$ & M $1-17$ & $228+051$ & 49,3 & 5,8 & 10,8 & 073800,8 & $-112529,6$ & $\mathrm{~A}, \mathrm{~B}$ \\
\hline $231.4+04.3$ & M $1-18$ & $231+041$ & 18,0 & 2,1 & 4,4 & 073945,8 & $-141406,1$ & $\mathrm{~A}, \mathrm{~B}$ \\
\hline $231.8+04.1$ & NGC 2438 & $231+042$ & 75,4 & 1,5 & 1,2 & 073932,6 & $-143701,4$ & A, B \\
\hline
\end{tabular}

Continua na próxima página. . 
Tabela A.1 - Continuação

\begin{tabular}{|c|c|c|c|c|c|c|c|c|}
\hline $\mathrm{PN} \mathrm{G}$ & Nome & PK & $V_{r}($ hel $)$ & $d_{\mathrm{M} 84}$ & $d_{\mathrm{SSV} 08}$ & $\alpha(1950)$ & $\delta(1950)$ & Amostra \\
\hline $232.0+05.7$ & SaSt $2-3$ & $232+051$ & 166,0 & & & 074544,9 & $-140009,7$ & B \\
\hline $232.4-01.8$ & M $1-13$ & $232-011$ & 45,5 & 4,5 & 5,4 & 071901,8 & $-180251,3$ & $\mathrm{~A}, \mathrm{~B}$ \\
\hline $232.8-04.7$ & M 1-11 & $232-041$ & 16,3 & & & 070905,9 & $-194600,4$ & $\mathrm{~A}, \mathrm{~B}$ \\
\hline $233.5-16.3$ & A 15 & $233-161$ & 35,0 & 3,1 & 3,7 & 062459,6 & $-252056,1$ & B \\
\hline $234.3-06.6$ & $\mathrm{~K} 2-3$ & $234-061$ & 65,2 & & & 070449,0 & $-215742,0$ & $\mathrm{~B}$ \\
\hline $234.8+02.4$ & NGC 2440 & $234+021$ & 62,7 & 1,1 & 1,4 & 073941,0 & $-180523,0$ & A, B \\
\hline $234.9-01.4$ & M 1-14 & $235-011$ & 77,4 & & 6,4 & 072545,6 & $-200712,0$ & $\mathrm{~A}, \mathrm{~B}$ \\
\hline $235.3-03.9$ & M $1-12$ & $235-031$ & 95,8 & & & 071712,8 & $-213819,7$ & $\mathrm{~A}, \mathrm{~B}$ \\
\hline $236.7+03.5$ & $\mathrm{~K} 1-12$ & $236+031$ & 58,8 & 3,9 & 2,7 & 074758,6 & $-191037,2$ & B \\
\hline $238.0+34.8$ & A 33 & $238+341$ & 60,1 & 1,6 & 0,8 & 093637,2 & $-023456,4$ & B \\
\hline $238.9+07.3$ & Sa $2-21$ & $238+072$ & & & 3,4 & 080630,3 & $-190513,4$ & A \\
\hline $239.6+13.9$ & NGC 2610 & $239+131$ & 88,9 & 2,6 & 2,2 & 083104,8 & $-155839,6$ & $\mathrm{~A}, \mathrm{~B}$ \\
\hline $240.3+07.0$ & $\mathrm{YC} 2-5$ & $240+071$ & & & 7,8 & 080828,9 & $-202235,0$ & A \\
\hline $240.3-07.6$ & M 3-2 & $240-071$ & 67,7 & 3,2 & 8,9 & 071249,6 & $-274506,0$ & A, B \\
\hline $241.0+02.3$ & M 3-4 & $241+021$ & 74,0 & 9,6 & 6,4 & 075303,3 & $-233014,0$ & $\mathrm{~A}, \mathrm{~B}$ \\
\hline $242.6-11.6$ & M 3-1 & $242-111$ & 56,8 & 4,3 & 4,6 & 070055,7 & $-313102,1$ & $\mathrm{~A}, \mathrm{~B}$ \\
\hline $243.3-01.0$ & NGC 2452 & $243-011$ & 62,0 & 2,7 & 2,8 & 074523,3 & $-271237,8$ & $\mathrm{~A}, \mathrm{~B}$ \\
\hline $245.4+01.6$ & M 3-5 & $245+011$ & 64,0 & 4,9 & 7,2 & 080025,4 & $-273328,3$ & A, B \\
\hline $253.5+10.7$ & $\mathrm{~K} 1-2$ & $253+101$ & 66,0 & 3,3 & & 085538,7 & $-284558,3$ & B \\
\hline $253.9+05.7$ & M 3-6 & $254+051$ & 45,8 & 2,6 & 4,2 & 083838,9 & $-321150,5$ & $\mathrm{~A}, \mathrm{~B}$ \\
\hline $258.1-00.3$ & $\mathrm{Sa} 2-26 / \mathrm{He} 2-9$ & $258-001$ & 53,1 & 0,8 & & 082638,0 & $-391338,2$ & $\mathrm{~A}, \mathrm{~B}$ \\
\hline $261.0+32.0$ & NGC 3242 & $261+321$ & 4,4 & 0,8 & 1,1 & 102221,6 & $-182317,9$ & $\mathrm{~A}, \mathrm{~B}$ \\
\hline $261.6+03.0$ & $\mathrm{Sa} 2-32 / \mathrm{He} 2-15$ & $261+021$ & 16,4 & 2,1 & 2,2 & 085137,8 & $-395216,6$ & $\mathrm{~A}, \mathrm{~B}$ \\
\hline $261.9+08.5$ & NGC 2818 & $261+081$ & $-0,9$ & 2,3 & 2,0 & 091400,5 & $-362505,1$ & $\mathrm{~A}, \mathrm{~B}$ \\
\hline $264.1-08.1$ & He $2-7$ & $264-081$ & 88,0 & 3,2 & 1,7 & 081002,0 & $-483416,2$ & $\mathrm{~A}, \mathrm{~B}$ \\
\hline $264.4-12.7$ & He $2-5$ & $264-121$ & 133,0 & 5,4 & 9,7 & 074600,5 & $-510733,5$ & $\mathrm{~A}, \mathrm{~B}$ \\
\hline $265.1-04.2$ & ESO 259-10 & $265-041$ & 49,0 & & & 083229,9 & $-470615,5$ & $\mathrm{~B}$ \\
\hline $265.7+04.1$ & NGC 2792 & $265+041$ & 14,0 & 1,8 & 3,1 & 091033,6 & $-421315,4$ & $\mathrm{~A}, \mathrm{~B}$ \\
\hline $268.4+02.4$ & PB 5 & $268+021$ & 53,3 & & & 091420,4 & $-451608,4$ & $\mathrm{~B}$ \\
\hline $269.7-03.6$ & PB 3 & $269-031$ & & 3,2 & 4,9 & 085243,5 & $-502054,6$ & A \\
\hline $272.1+12.3$ & NGC 3132 & $272+121$ & $-16,0$ & 1,1 & 1,3 & 100455,1 & $-401130,4$ & $\mathrm{~A}, \mathrm{~B}$ \\
\hline $273.2-03.7$ & He $2-18$ & $273-031$ & 40,0 & 3,1 & & 090707,3 & $-530701,1$ & $\mathrm{~B}$ \\
\hline $274.3+09.1$ & Lo 4 & $274+091$ & 33,0 & & & 100343,4 & $-440654,9$ & $\mathrm{~B}$ \\
\hline $274.6+02.1$ & Sa $2-48 /$ He $2-35$ & $274+022$ & 184,3 & 5,9 & 7,4 & 093949,0 & $-494415,6$ & $\mathrm{~B}$ \\
\hline $274.6+03.5$ & He $2-37$ & $274+031$ & 12,0 & 2,3 & 3,0 & 094533,3 & $-484414,9$ & $\mathrm{~A}, \mathrm{~B}$ \\
\hline $275.0-04.1$ & PB 4 & $275-041$ & & 2,9 & 3,7 & 091336,6 & $-544012,2$ & A \\
\hline $275.3-04.7$ & He $2-21$ & $275-042$ & 131,3 & 7,2 & 12,5 & 091223,5 & $-551548,5$ & $\mathrm{~A}, \mathrm{~B}$ \\
\hline $275.5-01.3$ & Pe $2-4$ & $275-011$ & 17,0 & 1,9 & & 092908,8 & $-525644,5$ & $\mathrm{~B}$ \\
\hline $275.8-02.9$ & He $2-29$ & $275-022$ & 25,0 & 4,0 & 4,0 & 092311,1 & $-542316,5$ & A, B \\
\hline $277.1-03.8$ & NGC 2899 & $277-031$ & 3,4 & 1,5 & 1,0 & 092530,9 & $-5553 \quad 15,8$ & $\mathrm{~A}, \mathrm{~B}$ \\
\hline $278.1-05.9$ & NGC 2867 & $278-051$ & 12,9 & 1,6 & 2,2 & 092000,6 & $-5805 \quad 50,7$ & $\mathrm{~A}, \mathrm{~B}$ \\
\hline $278.6-06.7$ & He $2-26$ & $278-061$ & 98,8 & 4,7 & 2,0 & 091806,0 & $-585915,8$ & $\mathrm{~B}$ \\
\hline $278.8+04.9$ & $\mathrm{Sa} 2-58 / \mathrm{PB} 6$ & $278+051$ & 58,7 & 4,0 & 4,4 & 101118,6 & $-500505,3$ & $\mathrm{~A}, \mathrm{~B}$ \\
\hline $279.6-03.1$ & He $2-36$ & $279-031$ & $-7,1$ & 2,7 & 2,4 & 094150,3 & $-570308,2$ & $\mathrm{~B}$ \\
\hline $280.0+02.9$ & Sa $2-56$ & $280+021$ & 71,9 & & & 101003,8 & $-522325,8$ & B \\
\hline
\end{tabular}

Continua na próxima página... 
Tabela A.1 - Continuação

\begin{tabular}{|c|c|c|c|c|c|c|c|c|}
\hline PN G & Nome & PK & $V_{r}($ hel $)$ & $d_{\mathrm{M} 84}$ & $d_{\mathrm{SSV} 08}$ & $\alpha(1950)$ & $\delta(1950)$ & Amostra \\
\hline $281.0-05.6$ & IC 2501 & $281-051$ & 31,5 & 2,0 & & 093720,5 & $-595154,2$ & $\mathrm{~A}, \mathrm{~B}$ \\
\hline $282.9+03.8$ & $\mathrm{He} 2-48$ & $282+031$ & & 5,1 & & 102932,5 & $-531803,7$ & A \\
\hline $283.3+03.9$ & He $2-50$ & $283+031$ & 10,9 & 2,8 & 5,2 & 103218,8 & $-532531,5$ & B \\
\hline $283.6+25.3$ & K $1-22$ & $283+251$ & $-14,0$ & & 1,0 & 112416,9 & $-340540,3$ & B \\
\hline $283.8-04.2$ & He $2-39$ & $283-041$ & $-23,0$ & 3,4 & & 100213,4 & $-602913,6$ & B \\
\hline $283.9-01.8$ & Hf 4 & $283-011$ & 22,0 & 1,9 & & 101350,7 & $-583613,0$ & B \\
\hline $285.4+01.5$ & Pe $1-1$ & $285+011$ & 19,8 & 2,2 & & 103629,7 & $-563128,3$ & B \\
\hline $285.4+02.2$ & $\operatorname{Pe} 2-7$ & $285+021$ & 26,5 & & & 103919,6 & $-555333,7$ & B \\
\hline $285.4-05.3$ & IC 2553 & $285-051$ & 37,3 & 2,8 & 3,7 & 100747,0 & $-622202,2$ & B \\
\hline $285.6-02.7$ & He $2-47$ & $285-021$ & $-17,7$ & 2,0 & & 102124,1 & $-601729,6$ & $\mathrm{~A}, \mathrm{~B}$ \\
\hline $285.7+01.2$ & Pe $1-2$ & $285+012$ & 73,2 & 2,1 & & 103734,7 & $-565034,0$ & B \\
\hline $285.7-14.9$ & IC 2448 & $285-141$ & $-24,0$ & 2,9 & 4,0 & 090637,3 & $-694420,8$ & $\mathrm{~A}, \mathrm{~B}$ \\
\hline $286.0-06.5$ & He $2-41$ & $286-061$ & 95,9 & 3,6 & & 100554,3 & $-633947,8$ & $\mathrm{~B}$ \\
\hline $286.3+02.8$ & $\mathrm{Sa} 2-68 / \mathrm{He} 2-55$ & $286+021$ & $-21,0$ & 2,6 & & 104640,1 & $-554717,2$ & $\mathrm{~A}, \mathrm{~B}$ \\
\hline $286.3-04.8$ & NGC 3211 & $286-041$ & $-22,3$ & 2,5 & 2,9 & 101612,7 & $\begin{array}{lll}-62 & 25 & 09,1\end{array}$ & $\mathrm{~A}, \mathrm{~B}$ \\
\hline $286.8-29.5$ & K $1-27$ & $286-291$ & 75,0 & & & 055850,1 & $-754031,8$ & $\mathrm{~B}$ \\
\hline $288.4+00.3$ & Hf 38 & $288+001$ & 64,0 & 2,2 & & 105233,5 & $-585346,6$ & $\mathrm{~B}$ \\
\hline $288.8-05.2$ & He $2-51$ & $288-051$ & 8,0 & 3,0 & 4,4 & 103402,1 & $-640337,4$ & $\mathrm{~A}, \mathrm{~B}$ \\
\hline $289.8+07.7$ & He $2-63$ & $289+071$ & 123,2 & 7,8 & 11,6 & 112141,5 & $-523450,0$ & $\mathrm{~B}$ \\
\hline $290.5+07.9$ & $\mathrm{Fg} 1$ & $290+071$ & 28,6 & 2,4 & 3,1 & 112614,9 & $-523932,1$ & $\mathrm{~A}, \mathrm{~B}$ \\
\hline $291.3-26.2$ & Vo 1 & & $-55,9$ & & & 070244,3 & $-793423,0$ & B \\
\hline $291.6-04.8$ & IC 2621 & $291-041$ & 11,4 & 2,1 & 4,9 & 105823,9 & $-645850,1$ & $\mathrm{~A}, \mathrm{~B}$ \\
\hline $291.7+03.7$ & He $2-64$ & $291+031$ & 72,0 & 4,6 & & 112505,8 & $-570127,2$ & $\mathrm{~B}$ \\
\hline $292.4+04.1$ & Sa $2-77 / \mathrm{PB} 8$ & $292+041$ & 21,9 & 5,1 & 7,3 & 113056,4 & $-564938,9$ & B \\
\hline $292.6+01.2$ & NGC 3699 & $292+011$ & $-15,8$ & 2,0 & 1,6 & 112540,6 & $-594055,7$ & B \\
\hline $292.8+01.1$ & He $2-67$ & $292+012$ & 59,5 & 4,3 & & 112629,9 & $\begin{array}{lll}-59 & 50 & 04,7\end{array}$ & $\mathrm{~A}, \mathrm{~B}$ \\
\hline $293.6+10.9$ & $\mathrm{BlDz} 1$ & $293+101$ & $-10,0$ & & & 115035,8 & $-503417,3$ & B \\
\hline $294.1+43.6$ & NGC 4361 & $294+431$ & 9,3 & 0,9 & 0,9 & 122154,7 & $-183028,2$ & $\mathrm{~A}, \mathrm{~B}$ \\
\hline $294.6+04.7$ & NGC 3918 & $294+041$ & $-16,1$ & 0,9 & 1,6 & 114748,9 & $-565415,5$ & $\mathrm{~A}, \mathrm{~B}$ \\
\hline $294.9-04.3$ & He $2-68$ & $294-041$ & 40,5 & 5,1 & & 112930,7 & $-654139,3$ & B \\
\hline $295.3-09.3$ & He $2-62$ & $295-091$ & 89,8 & & & 111545,2 & $-703307,6$ & B \\
\hline $296.3-03.0$ & He $2-73$ & $296-031$ & $-1,3$ & 3,4 & 6,7 & 114612,3 & $-645156,3$ & B \\
\hline $296.4-06.9$ & He $2-71$ & $296-061$ & 57,9 & 7,8 & & 113653,9 & $-683530,9$ & B \\
\hline $296.6-20.0$ & NGC 3195 & $296-201$ & $-7,3$ & 2,4 & 2,0 & 100956,9 & $-803642,1$ & $\mathrm{~A}, \mathrm{~B}$ \\
\hline $297.4+03.7$ & He $2-78$ & $297+031$ & 25,8 & 3,8 & & 120632,7 & $-582555,6$ & B \\
\hline $298.3+06.6$ & Po 1 & $298+061$ & $-38,0$ & & & 121551,6 & $-553724,7$ & B \\
\hline $298.3-04.8$ & NGC 4071 & $298-041$ & 11,0 & 2,4 & 1,6 & 120139,0 & $-670153,1$ & B \\
\hline $299.5+02.4$ & He $2-82$ & $299+021$ & $-12,5$ & 2,0 & & 122108,4 & $-595635,9$ & B \\
\hline $300.4-00.9$ & He $2-84$ & $300-001$ & $-44,0$ & 1,6 & & 122557,0 & $-632801,8$ & B \\
\hline $300.7-02.0$ & $\mathrm{Sa} 2-86 / \mathrm{He} 2-86$ & $300-021$ & $-7,5$ & 2,6 & & 122738,9 & $-643530,7$ & $\mathrm{~A}, \mathrm{~B}$ \\
\hline $300.8-03.4$ & ESO 095-12 & $300-031$ & 57,0 & & & 122731,9 & $-655748,6$ & $\mathrm{~B}$ \\
\hline $302.3-01.3$ & DuRe 1 & & 55,1 & & & 124251,1 & $-635314,2$ & $\mathrm{~B}$ \\
\hline $303.6+40.0$ & A 35 & $303+401$ & $-6,6$ & 0,5 & 0,2 & 125052,9 & $-223605,4$ & $\mathrm{~B}$ \\
\hline $304.2+05.9$ & Wray $16-122$ & $304+052$ & $-30,0$ & & & 125741,4 & $-563730,9$ & B \\
\hline $304.5-04.8$ & IC 4191 & $304-041$ & $-18,3$ & 2,0 & 2,7 & 130527,0 & $-672237,7$ & B \\
\hline
\end{tabular}

Continua na próxima página... 
Tabela A.1 - Continuação

\begin{tabular}{|c|c|c|c|c|c|c|c|c|}
\hline $\mathrm{PN}$ G & Nome & PK & $V_{r}($ hel $)$ & $d_{\mathrm{M} 84}$ & $d_{\mathrm{SSV} 08}$ & $\alpha(1950)$ & $\delta(1950)$ & Amostra \\
\hline $304.8+05.1$ & He $2-88$ & $304+051$ & $-4,8$ & & & 130245,4 & $-572321,0$ & B \\
\hline $305.6-13.1$ & ESO $040-11$ & $305-131$ & $-16,0$ & & & 132958,4 & $-753108,7$ & B \\
\hline $306.4-00.6$ & $\mathrm{Th} 2-\mathrm{A}$ & $306-001$ & $-45,0$ & 2,5 & 2,5 & 131914,3 & $-630520,5$ & $\mathrm{~A}, \mathrm{~B}$ \\
\hline $307.2-03.4$ & NGC 5189 & $307-031$ & $-9,5$ & 0,7 & 0,5 & 133001,0 & $-654303,8$ & $\mathrm{~A}, \mathrm{~B}$ \\
\hline $307.2-09.0$ & He $2-97$ & $307-091$ & 51,6 & 4,9 & & 134122,3 & $-71 \quad 1353,4$ & B \\
\hline $307.5-04.9$ & My Cn 18 & $307-041$ & $-67,8$ & 2,8 & 3,2 & 133555,4 & $-670739,1$ & B \\
\hline $309.0+00.8$ & He $2-96$ & $309+001$ & $-53,2$ & 0,9 & & 133910,5 & $-610722,2$ & B \\
\hline $309.0-04.2$ & He $2-99$ & $309-041$ & 1,2 & 4,2 & 3,8 & 134846,3 & $-660838,8$ & $\mathrm{~A}, \mathrm{~B}$ \\
\hline $309.1-04.3$ & NGC 5315 & $309-042$ & $-34,5$ & 1,2 & & 135011,4 & $-661605,6$ & $\mathrm{~A}, \mathrm{~B}$ \\
\hline $309.2+01.3$ & VBRC 5 & $309+011$ & $-59,0$ & & & 134034,9 & $-603442,5$ & B \\
\hline $309.5-02.9$ & $\mathrm{MaC} 1-2$ & $302-021$ & $-47,1$ & & & 135046,1 & $-644452,2$ & B \\
\hline $310.3+24.7$ & Lo 8 & $310+241$ & $-3,0$ & & & 132245,4 & $-372039,3$ & $\mathrm{~B}$ \\
\hline $310.7-02.9$ & He $2-103$ & $310-021$ & $-29,9$ & 4,0 & 3,4 & 140150,9 & $-642640,0$ & B \\
\hline $311.0+02.4$ & SuWt 2 & $311+022$ & $-40,0$ & & & 135215,9 & $-590758,8$ & B \\
\hline $311.1+03.4$ & He $2-101$ & $311+031$ & $-40,8$ & & & 135130,6 & $-581233,9$ & $\mathrm{~B}$ \\
\hline $312.0-02.0$ & He $2-106$ & $312-021$ & 8,0 & & & 141023,1 & $-631146,1$ & B \\
\hline $312.3+10.5$ & NGC 5307 & $312+101$ & 39,9 & 2,3 & 3,2 & 134751,5 & $-505730,4$ & $\mathrm{~A}, \mathrm{~B}$ \\
\hline $315.0-00.3$ & He $2-111$ & $315-001$ & $-10,4$ & 2,8 & 3,5 & 142931,5 & $-603623,4$ & $\mathrm{~A}, \mathrm{~B}$ \\
\hline $315.1-13.0$ & He $2-131$ & $315-131$ & $-1,2$ & 1,6 & & 153153,5 & $-714458,2$ & $\mathrm{~A}, \mathrm{~B}$ \\
\hline $315.4+09.4$ & He $2-104$ & $315+091$ & $-143,6$ & 6,3 & & 140833,4 & $-511219,1$ & $\mathrm{~B}$ \\
\hline $316.1+08.4$ & He $2-108$ & $316+081$ & $-11,1$ & 3,9 & 4,3 & 141447,0 & $-515649,5$ & $\mathrm{~A}, \mathrm{~B}$ \\
\hline $317.1-05.7$ & He $2-119$ & $317-051$ & $-11,0$ & 1,7 & 1,4 & 150623,9 & $-642906,4$ & $\mathrm{~A}, \mathrm{~B}$ \\
\hline $318.3-02.0$ & He $2-114$ & $318-021$ & $-37,0$ & 4,1 & 2,6 & 150009,7 & $-604136,5$ & $\mathrm{~B}$ \\
\hline $318.4+41.4$ & A 36 & $318+411$ & 36,8 & 0,6 & 0,4 & 133757,4 & $-193745,9$ & $\mathrm{~B}$ \\
\hline $319.2+06.8$ & $\mathrm{He} 2-112$ & $319+061$ & & 2,5 & 3,0 & 143701,6 & $-522205,3$ & A \\
\hline $319.6+15.7$ & IC 4406 & $319+151$ & $-41,4$ & 1,7 & 2,4 & 141915,3 & $-435525,3$ & A, B \\
\hline $320.1-09.6$ & He $2-138$ & $320-091$ & $-56,9$ & 3,1 & 4,8 & 155119,6 & $-660023,8$ & $\mathrm{~A}, \mathrm{~B}$ \\
\hline $320.3-28.8$ & He $2-434$ & $320-281$ & 38,0 & & & 192731,6 & $-743924,8$ & B \\
\hline $320.9+02.0$ & He $2-117$ & $321+022$ & 44,6 & 1,7 & & 150214,4 & $-554740,4$ & $\mathrm{~A}, \mathrm{~B}$ \\
\hline $321.0+03.9$ & He $2-113$ & $321+031$ & $-56,9$ & & & 145614,7 & $-540612,6$ & $\mathrm{~B}$ \\
\hline $321.3+02.8$ & He $2-115$ & $321+021$ & $-56,2$ & 2,0 & & 150134,3 & $-545932,3$ & $\mathrm{~A}, \mathrm{~B}$ \\
\hline $321.3-16.7$ & He $2-185$ & $321-161$ & $-6,0$ & 4,9 & & 165545,8 & $-700137,4$ & B \\
\hline $321.8+01.9$ & He $2-120$ & $321+011$ & $-18,4$ & 3,2 & 2,7 & 150810,9 & $-552829,5$ & B \\
\hline $322.1-06.6$ & He $2-136$ & $322-061$ & $-135,0$ & 4,5 & & 154749,0 & $-622147,9$ & B \\
\hline $322.4-00.1$ & $\operatorname{Pe} 2-8$ & $322-001$ & $-16,3$ & 2,9 & & 151949,6 & $-565846,7$ & B \\
\hline $322.4-02.6$ & Mz 1 & $322-021$ & $-32,3$ & 2,4 & 2,3 & 153014,1 & $-585906,6$ & $\mathrm{~B}$ \\
\hline $322.5-05.2$ & NGC 5979 & $322-051$ & 23,0 & 2,6 & 4,1 & 154326,4 & $-610350,6$ & $\mathrm{~A}, \mathrm{~B}$ \\
\hline $323.1-02.5$ & He $2-132$ & $323-021$ & $-131,0$ & 3,7 & 3,4 & 153358,6 & $-583452,7$ & B \\
\hline $323.6-04.5$ & IRAS $15463-5949$ & $323-041$ & $-41,0$ & & & 154618,3 & $-594945,4$ & $\mathrm{~B}$ \\
\hline $323.9+02.4$ & He $2-123$ & $323+021$ & $-12,0$ & 2,9 & 5,8 & 151834,9 & $-535730,2$ & $\mathrm{~A}, \mathrm{~B}$ \\
\hline $324.0+03.5$ & PM 1-89 & $324+031$ & $-81,0$ & & & 151527,6 & $-525856,4$ & $\mathrm{~B}$ \\
\hline $324.1+09.0$ & ESO $223-10$ & $324+091$ & $-55,0$ & & & 145814,0 & $-480913,0$ & $\mathrm{~B}$ \\
\hline $324.2+02.5$ & He $2-125$ & $324+021$ & $-37,1$ & 1,8 & 10,4 & 151952,3 & $-534049,5$ & $\mathrm{~B}$ \\
\hline $325.0+03.2$ & He $2-129$ & $325+031$ & $-16,3$ & 10,3 & & 152150,7 & $-524006,0$ & $\mathrm{~B}$ \\
\hline $325.4-04.0$ & He $2-141$ & $325-041$ & $-46,0$ & 2,8 & 3,5 & 155501,9 & $-581520,9$ & $\mathrm{~A}, \mathrm{~B}$ \\
\hline
\end{tabular}

Continua na próxima página... 
Tabela A.1 - Continuação

\begin{tabular}{|c|c|c|c|c|c|c|c|c|}
\hline PN G & Nome & PK & $V_{r}($ hel $)$ & $d_{\mathrm{M} 84}$ & $d_{\mathrm{SSV} 08}$ & $\alpha(1950)$ & $\delta(1950)$ & Amostra \\
\hline $325.8+04.5$ & He $2-128$ & $325+041$ & $-94,8$ & 4,7 & & 152129,5 & $-510909,0$ & $\mathrm{~B}$ \\
\hline $325.8-12.8$ & He $2-182$ & $325-121$ & $-89,5$ & 3,8 & & 164949,1 & $-640936,2$ & B \\
\hline $326.0-06.5$ & He $2-151$ & $326-061$ & $-128,2$ & 8,4 & & 161125,7 & $-594631,9$ & B \\
\hline $326.7+42.2$ & IC 972 & $326+421$ & $-26,6$ & 3,8 & 2,5 & 140141,6 & $-165919,2$ & B \\
\hline $327.1-01.8$ & He $2-140$ & $327-012$ & $-70,2$ & 2,9 & & 155410,6 & $-553314,5$ & $\mathrm{~A}, \mathrm{~B}$ \\
\hline $327.1-02.2$ & He $2-142$ & $327-021$ & $-94,5$ & 3,7 & 7,4 & 155558,9 & $-554703,9$ & B \\
\hline $327.5+13.3$ & He $2-118$ & $327+131$ & $-134,2$ & 8,4 & & 150255,2 & $-424821,8$ & $\mathrm{~A}, \mathrm{~B}$ \\
\hline $327.8+10.0$ & NGC 5882 & $327+101$ & 9,7 & 1,6 & 2,4 & 151324,9 & $-452757,8$ & $\mathrm{~A}, \mathrm{~B}$ \\
\hline $327.8-01.6$ & He $2-143$ & $327-011$ & $-35,0$ & 2,7 & 5,3 & 155702,8 & $-545714,7$ & B \\
\hline $327.8-06.1$ & He $2-158$ & $327-061$ & $-39,0$ & 10,0 & 19,9 & 161918,6 & $-581224,7$ & $\mathrm{~A}, \mathrm{~B}$ \\
\hline $327.8-07.2$ & He $2-163$ & $327-071$ & $-45,0$ & 3,1 & 4,8 & 162514,9 & $-590250,8$ & B \\
\hline $328.9-02.4$ & He $2-146$ & $328-021$ & 61,0 & 1,7 & 2,0 & 160643,4 & $-544944,9$ & B \\
\hline $329.0+01.9$ & Sp 1 & $329+021$ & $-31,5$ & 1,6 & 1,2 & 154756,5 & $-5122 \quad 29,2$ & B \\
\hline $329.3-02.8$ & $\mathrm{Mz} 2$ & $329-022$ & $-29,7$ & 2,3 & 2,4 & 161033,9 & $-544931,2$ & $\mathrm{~A}, \mathrm{~B}$ \\
\hline $329.4-02.7$ & He $2-149$ & $329-021$ & $-113,0$ & 8,4 & 13,0 & 161026,2 & $-544005,3$ & B \\
\hline $330.2+05.9$ & Lo 9 & $330+051$ & $-33,0$ & & & 153839,5 & $-4731 \quad 12,9$ & $\mathrm{~B}$ \\
\hline $330.6-02.1$ & He $2-153$ & $330-021$ & $-40,2$ & 1,2 & 7,7 & $1613 \quad 19,7$ & $-532446,1$ & $\mathrm{~A}, \mathrm{~B}$ \\
\hline $330.6-03.6$ & He $2-159$ & $330-031$ & $-89,2$ & 4,3 & 4,9 & 162022,2 & $-542908,9$ & B \\
\hline $330.7+04.1$ & Cn $1-1$ & $330+041$ & $-9,0$ & & & 154738,2 & $-483557,8$ & B \\
\hline $330.9+04.3$ & Wray 16-189 & $330+042$ & 27,0 & & & 154742,7 & $-481706,5$ & B \\
\hline $331.0-02.7$ & He $2-157$ & $331-021$ & $-70,0$ & 5,4 & 9,6 & 161818,2 & $-533351,4$ & $\mathrm{~A}, \mathrm{~B}$ \\
\hline $331.1-05.7$ & PC 11 & $331-051$ & $-52,0$ & 6,2 & & 163337,6 & $-553625,7$ & $\mathrm{~B}$ \\
\hline $331.3+16.8$ & NGC 5873 & $331+161$ & $-130,5$ & 3,8 & 5,4 & 150938,5 & $-375620,4$ & $\mathrm{~A}, \mathrm{~B}$ \\
\hline $331.3-12.1$ & He $3-1357$ & $331-121$ & 13,0 & & & 171156,3 & $-592603,8$ & B \\
\hline $331.4-03.5$ & He $2-162$ & $331-031$ & 30,6 & 5,6 & & 162352,9 & $-535448,1$ & B \\
\hline $331.5-02.7$ & He $2-161$ & $331-022$ & $-98,0$ & 4,0 & 5,3 & 162042,3 & $-531541,0$ & B \\
\hline $331.5-03.9$ & He $2-165$ & $331-032$ & $-17,7$ & 1,6 & 2,0 & 162601,3 & $-540256,3$ & B \\
\hline $331.7-01.0$ & Mz 3 & $331-011$ & $-21,2$ & 1,0 & 1,4 & 161323,1 & $-515148,4$ & $\mathrm{~A}, \mathrm{~B}$ \\
\hline $332.0-03.3$ & He $2-164$ & $332-031$ & $-73,0$ & 2,3 & 2,8 & 162556,9 & $-531643,3$ & $\mathrm{~A}, \mathrm{~B}$ \\
\hline $332.3-04.2$ & He $2-170$ & $332-041$ & 60,6 & 7,1 & & 163122,6 & $-534401,1$ & B \\
\hline $332.9-09.9$ & Не $3-1333$ & $332-091$ & $-65,6$ & & & 170447,8 & $-565056,1$ & B \\
\hline $333.4+01.1$ & He $2-152$ & $333+011$ & $-63,5$ & 2,0 & 3,0 & 161137,2 & $-490552,2$ & B \\
\hline $333.4-04.0$ & Ha $\operatorname{Tr} 3$ & $333-041$ & $-88,0$ & & & 163541,5 & $-524320,7$ & B \\
\hline $334.3-09.3$ & IC 4642 & $334-091$ & 43,9 & 2,7 & 3,0 & 170736,8 & $-552023,1$ & B \\
\hline $334.8-07.4$ & SaSt $2-12$ & $334-071$ & $-63,0$ & & & 165900,5 & $-535138,5$ & B \\
\hline $335.2-03.6$ & $\mathrm{HaTr} 4$ & $335-031$ & $-97,0$ & & & 164108,1 & $-510650,7$ & $\mathrm{~B}$ \\
\hline $335.4+09.2$ & ESO 330-02 & $335+091$ & $-62,0$ & & & 154948,2 & $-414133,1$ & B \\
\hline $335.6-04.0$ & MeWe 1-8 & $335-041$ & 24,0 & & & 164447,8 & $-510405,2$ & $\mathrm{~B}$ \\
\hline $335.9-03.6$ & MeWe $1-7$ & $335-032$ & $-85,0$ & & & 164405,9 & $-503730,3$ & B \\
\hline $336.2+01.9$ & Pe $1-6$ & $336+011$ & $-76,0$ & 4,0 & 5,4 & 162016,1 & $-463519,9$ & $\mathrm{~B}$ \\
\hline $336.2-06.9$ & $\mathrm{PC} 14$ & $336-061$ & $-46,0$ & 4,5 & 5,8 & 170216,5 & $-522559,0$ & $\mathrm{~A}, \mathrm{~B}$ \\
\hline $336.3-05.6$ & He $2-186$ & $336-051$ & $-72,3$ & 6,2 & 10,4 & 165540,9 & $-513738,9$ & B \\
\hline $336.8-07.2$ & K $2-17$ & $336-071$ & 8,0 & & & 170538,2 & $-520915,2$ & B \\
\hline $336.9+08.3$ & StWr $4-10$ & $336+081$ & $-159,0$ & & & 155848,0 & $-4125 \quad 16,9$ & B \\
\hline $336.9-11.5$ & MeWe $1-10$ & $336-111$ & $-14,0$ & & & 173021,5 & $-542657,4$ & $\mathrm{~B}$ \\
\hline
\end{tabular}

Continua na próxima página. . 
Tabela A.1 - Continuação

\begin{tabular}{|c|c|c|c|c|c|c|c|c|}
\hline PN G & Nome & PK & $V_{r}($ hel $)$ & $d_{\mathrm{M} 84}$ & $d_{\mathrm{SSV} 08}$ & $\alpha(1950)$ & $\delta(1950)$ & Amostra \\
\hline $337.4+01.6$ & Pe $1-7$ & $337+011$ & $-33,0$ & 2,6 & & 162648,3 & $-455621,6$ & B \\
\hline $337.4-09.1$ & Wray 16-266 & $337-091$ & $-101,0$ & & & 171836,6 & $-524343,0$ & $\mathrm{~B}$ \\
\hline $337.5-05.1$ & He $2-187$ & $337-051$ & 52,0 & 3,2 & & 165745,3 & $-501836,7$ & $\mathrm{~B}$ \\
\hline $337.6-04.2$ & MeWe $1-9$ & $337-041$ & $-96,0$ & & & 165339,3 & $-494216,6$ & $\mathrm{~B}$ \\
\hline $338.1-08.3$ & NGC 6326 & $338-081$ & 8,5 & 2,5 & 3,5 & 171649,2 & $-514216,0$ & $\mathrm{~B}$ \\
\hline $338.8+05.6$ & He $2-155$ & $338+051$ & $-35,3$ & 2,7 & 3,1 & 161554,7 & $-420823,7$ & $\mathrm{~B}$ \\
\hline $340.4-14.1$ & $\mathrm{Sa} 1-6$ & $340-141$ & $-279,0$ & & & 175657,8 & $-524416,1$ & $\mathrm{~B}$ \\
\hline $340.8+10.8$ & Lo 12 & $340+101$ & 9,0 & & & 160508,3 & $-370049,7$ & $\mathrm{~B}$ \\
\hline $340.8+12.3$ & Lo 11 & $340+121$ & $-79,0$ & & & 160006,3 & $-355239,1$ & $\mathrm{~B}$ \\
\hline $340.9-04.6$ & $\mathrm{Sa} 1-5$ & $340-041$ & $-2,0$ & & & 170742,7 & $-472123,1$ & $\mathrm{~B}$ \\
\hline $341.0+09.4$ & SB25 & $341+091$ & $-93,0$ & & & 161018,1 & $-375223,2$ & B \\
\hline $341.5-09.1$ & He $2-248$ & $341-091$ & $-25,0$ & 6,7 & & 173215,7 & $-492352,6$ & $\mathrm{~B}$ \\
\hline $341.6+13.7$ & NGC 6026 & $341+131$ & $-101,5$ & 1,9 & 2,4 & 155807,6 & $-342413,6$ & B \\
\hline $341.7-06.0$ & SB26 & $341-061$ & $-154,0$ & & & 171717,2 & $-473229,6$ & $\mathrm{~B}$ \\
\hline $341.8+05.4$ & NGC 6153 & $341+051$ & 38,8 & 1,0 & 1,5 & 162804,7 & $-4008 \quad 47,8$ & $\mathrm{~A}, \mathrm{~B}$ \\
\hline $341.9+08.8$ & SB27 & $341+081$ & $-2,0$ & & & 161553,6 & $-3740 \quad 14,7$ & $\mathrm{~B}$ \\
\hline $342.1+10.8$ & NGC 6072 & $342+101$ & 6,9 & 1,3 & 1,0 & 160940,8 & $-360608,3$ & $\mathrm{~B}$ \\
\hline $342.1+27.5$ & Me $2-1$ & $342+271$ & 44,4 & 4,0 & 6,7 & 151923,1 & $-232650,0$ & $\mathrm{~A}, \mathrm{~B}$ \\
\hline $342.3-06.0$ & SB28 & $342-062$ & $-27,0$ & & & 171908,2 & $-465958,9$ & B \\
\hline $342.5-14.3$ & Sp 3 & $342-141$ & 45,2 & 2,2 & 1,9 & 180319,4 & $-510133,2$ & $\mathrm{~B}$ \\
\hline $342.7+00.7$ & H $1-3$ & $342+001$ & $-6,0$ & 1,4 & 2,1 & 164958,8 & $-423429,3$ & $\mathrm{~B}$ \\
\hline $342.8-06.6$ & Cn $1-4$ & $342-061$ & $-87,4$ & & & 172404,4 & $-465312,8$ & B \\
\hline $342.9-02.0$ & Pe $1-8$ & $342-021$ & $-1,0$ & 2,2 & & 170245,7 & $-440909,8$ & $\mathrm{~B}$ \\
\hline $342.9-04.9$ & He $2-207$ & $342-041$ & $-36,1$ & & & 171551,5 & $-455012,7$ & $\mathrm{~B}$ \\
\hline $343.4+11.9$ & H $1-1$ & $343+111$ & 91,2 & 8,8 & 16,4 & 161013,4 & $-342803,5$ & B \\
\hline $343.5-07.8$ & PC 17 & $343-071$ & $-128,1$ & 4,8 & 8,2 & 173156,8 & $-465754,2$ & $\mathrm{~B}$ \\
\hline $343.6+03.7$ & SuWt 3 & $343+031$ & $-110,0$ & & & 164057,4 & $-395748,8$ & $\mathrm{~B}$ \\
\hline $343.7-09.6$ & SB29 & $343-091$ & $-88,0$ & & & 174146,4 & $-474240,1$ & $\mathrm{~B}$ \\
\hline $343.9-05.8$ & SB30 & $343-051$ & 67,0 & & & 172321,2 & $-453006,8$ & $\mathrm{~B}$ \\
\hline $344.2+04.7$ & $\mathrm{Vd} 1-1$ & $344+041$ & $-142,1$ & & & 163909,0 & $-384852,5$ & $\mathrm{~B}$ \\
\hline $344.2-01.2$ & $\mathrm{H} 1-6$ & $344-011$ & $-51,0$ & 1,4 & & 170325,3 & $-4237 \quad 12,3$ & $\mathrm{~B}$ \\
\hline $344.4-06.1$ & Wray $16-278$ & $344-061$ & $-35,0$ & & & 172623,1 & $-452032,1$ & $\mathrm{~B}$ \\
\hline $344.8+03.4$ & Vd $1-3$ & $344+031$ & $-138,8$ & & & 164607,0 & $-391558,6$ & B \\
\hline $345.0+03.4$ & Vd $1-4$ & $345+031$ & 35,1 & & & 164659,9 & $-390312,3$ & B \\
\hline $345.0+04.3$ & $\operatorname{Vd} 1-2$ & $345+041$ & 3,4 & & & 164320,9 & $-383136,1$ & $\mathrm{~B}$ \\
\hline $345.0-04.9$ & $\mathrm{Cn} 1-3$ & $345-041$ & $-79,8$ & & & 172234,4 & $-440850,3$ & B \\
\hline $345.2-08.8$ & Tc 1 & $345-081$ & $-84,1$ & 1,0 & 2,7 & 174152,3 & $-460411,9$ & $\mathrm{~A}, \mathrm{~B}$ \\
\hline $345.3-10.2$ & MeWe $1-11$ & $345-101$ & 21,0 & & & 174902,4 & $-464121,9$ & B \\
\hline $345.4+00.1$ & IC 4637 & $345+001$ & 10,4 & 1,4 & 2,4 & 170140,7 & $-404903,5$ & B \\
\hline $345.5+15.1$ & Lo 13 & $345+151$ & $-19,0$ & & & 160636,9 & $-304717,7$ & B \\
\hline $345.6+06.7$ & He $2-175$ & $345+061$ & $-33,6$ & 6,4 & 6,1 & 163608,0 & $-362824,3$ & B \\
\hline $345.9+03.0$ & $\mathrm{Vd} 1-6$ & $345+032$ & $-142,0$ & & & 165102,3 & $-383920,5$ & B \\
\hline $346.0+08.5$ & He $2-171$ & $346+081$ & $-101,5$ & & & 163047,0 & $-345913,0$ & $\mathrm{~B}$ \\
\hline $346.2-08.2$ & IC 4663 & $346-081$ & $-52,1$ & 3,2 & 3,5 & 174148,1 & $-445303,1$ & B \\
\hline $346.3-06.8$ & $\mathrm{Fg} 2$ & $346-061$ & 34,5 & & & 173541,5 & $-440759,3$ & B \\
\hline
\end{tabular}

Continua na próxima página... 
Tabela A.1 - Continuação

\begin{tabular}{|c|c|c|c|c|c|c|c|c|}
\hline PN G & Nome & $\mathrm{PK}$ & $V_{r}($ hel $)$ & $d_{\mathrm{M} 84}$ & $d_{\mathrm{SSV} 08}$ & $\alpha(1950)$ & $\delta(1950)$ & Amostra \\
\hline $346.9+12.4$ & K $1-3$ & $346+121$ & $-12,5$ & 3,0 & 0,6 & 162007,6 & $-313801,7$ & B \\
\hline $347.4+05.8$ & H $1-2$ & $347+051$ & $-105,5$ & & & 164534,6 & $-354156,1$ & B \\
\hline $347.7+02.0$ & $\mathrm{Vd} 1-8$ & $347+011$ & $-117,0$ & & & 170109,7 & $-374907,9$ & B \\
\hline $347.9-06.0$ & SB31 & $347-061$ & $-70,0$ & & & 173628,8 & $-422231,2$ & B \\
\hline $348.0+06.3$ & MGP 1 & $348+061$ & $-52,0$ & & & 164530,4 & $-345544,1$ & B \\
\hline $348.0-13.8$ & IC 4699 & $348-131$ & $-123,3$ & 5,2 & 7,7 & 181449,2 & $-460014,5$ & B \\
\hline $348.4-04.1$ & H $1-21$ & $348-041$ & $-200,7$ & & & 172916,5 & $-405622,3$ & B \\
\hline $348.8-09.0$ & He $2-306$ & $348-091$ & $-40,0$ & 7,1 & & 175257,5 & $-430256,2$ & B \\
\hline $349.2-03.5$ & H $2-14$ & $349-031$ & $-12,0$ & & & 172851,0 & $-394917,3$ & B \\
\hline $349.3-01.1$ & NGC 6337 & $349-011$ & $-70,9$ & 1,7 & 1,4 & 171849,6 & $-382611,7$ & B \\
\hline $349.3-04.2$ & Lo 16 & $349-041$ & 13,0 & & & 173212,1 & $-400932,6$ & B \\
\hline $349.5+01.0$ & NGC 6302 & $349+011$ & $-38,9$ & 0,4 & 0,7 & 171021,0 & $-370247,7$ & $\mathrm{~A}, \mathrm{~B}$ \\
\hline $349.7-09.1$ & SB32 & $349-091$ & 186,0 & & & 175552,1 & $-422442,0$ & B \\
\hline $349.8+04.4$ & M 2-4 & $349+041$ & $-207,2$ & & & 165747,6 & $-344516,8$ & B \\
\hline $350.1-03.9$ & H $1-26$ & $350-031$ & $-36,9$ & 2,5 & & 173301,7 & $-392006,6$ & B \\
\hline $350.5-05.0$ & H $1-28$ & $350-051$ & $-44,2$ & 1,7 & & 173925,2 & $-393501,7$ & B \\
\hline $350.8-02.4$ & H $1-22$ & $350-021$ & $-213,0$ & & & 172856,7 & $-375515,8$ & B \\
\hline $350.9+04.4$ & H $2-1$ & $350+041$ & $-18,8$ & 3,4 & 5,7 & 1701 18,9 & $-335511,9$ & B \\
\hline $351.0-10.4$ & HaTr 9 & $351-102$ & $-38,0$ & & & 180525,4 & $-414909,9$ & B \\
\hline $351.1+04.8$ & M 1-19 & $351+041$ & $-55,2$ & & 10,8 & 170030,3 & $-332533,3$ & B \\
\hline $351.2+05.2$ & M 2-5 & $351+051$ & $-123,4$ & 1,5 & & 165903,1 & $-330548,4$ & B \\
\hline $351.2-06.3$ & SB33 & $351-062$ & 58,0 & & & 174658,6 & $-393928,1$ & B \\
\hline $351.3+07.6$ & $\mathrm{H} 1-4$ & $351+071$ & 2,7 & & & 165023,9 & $-313540,0$ & B \\
\hline $351.5-06.5$ & SB34 & $351-063$ & $-57,0$ & & & 174840,5 & $-393132,6$ & B \\
\hline $351.6-06.2$ & H $1-37$ & $351-061$ & $-22,3$ & 3,9 & & 174716,2 & $-391637,9$ & B \\
\hline $351.7-06.6$ & SB35 & $351-064$ & $-120,0$ & & & 174934,2 & $-392330,9$ & $\mathrm{~B}$ \\
\hline $351.7-10.9$ & Wray $16-385$ & $351-101$ & $-58,0$ & & & 180920,0 & $-413115,2$ & B \\
\hline $351.9+09.0$ & PC 13 & $351+091$ & $-75,1$ & & & 164706,2 & $-301448,6$ & B \\
\hline $351.9-01.9$ & Wray $16-286$ & $351-011$ & $-152,3$ & & & 172937,4 & $-364146,7$ & $\mathrm{~B}$ \\
\hline $352.0-04.6$ & H $1-30$ & $352-041$ & $-12,9$ & 2,2 & & 174140,7 & $-3807 \quad 37,1$ & $\mathrm{~B}$ \\
\hline $352.0-06.7$ & SB36 & $352-061$ & 35,0 & & & 175052,6 & $-391005,6$ & B \\
\hline $352.1+05.1$ & M 2-8 & $352+051$ & 25,1 & 3,1 & 9,3 & 170215,6 & $-322805,1$ & $\mathrm{~B}$ \\
\hline $352.6+03.0$ & H $1-8$ & $352+032$ & $-116,0$ & 5,1 & 8,9 & 171126,0 & $-332123,6$ & B \\
\hline $352.6-04.9$ & SB37 & $352-042$ & 20,0 & & & 174427,2 & $-374702,6$ & $\mathrm{~B}$ \\
\hline $352.7-08.4$ & SB38 & $352-081$ & 59,0 & & & 180000,4 & $-392134,5$ & B \\
\hline $352.8-00.2$ & H $1-13$ & $352-001$ & $-26,3$ & 0,8 & & 172507,2 & $-350506,8$ & $\mathrm{~A}, \mathrm{~B}$ \\
\hline $352.9+11.4$ & $\mathrm{~K} 2-16$ & $352+112$ & 5,2 & & & 164141,7 & $-275835,3$ & B \\
\hline $352.9-07.5$ & $\mathrm{Fg} 3$ & $352-071$ & 4,0 & 2,0 & & 175644,3 & $-384945,9$ & B \\
\hline $353.0+08.3$ & MyCn 26 & $353+081$ & 44,0 & & & 165236,8 & $-294534,6$ & B \\
\hline $353.2-05.2$ & H $1-38$ & $353-051$ & 149,6 & 5,7 & & 174720,4 & $-372306,3$ & B \\
\hline $353.3+06.3$ & M 2-6 & $353+062$ & $-127,4$ & & & 170105,9 & $-304920,9$ & $\mathrm{~A}, \mathrm{~B}$ \\
\hline $353.3-08.3$ & SB39 & $353-081$ & 6,0 & & & 180104,2 & $-384751,4$ & B \\
\hline $353.4-02.4$ & K 5-8 & $353-022$ & $-52,3$ & & & 173555,5 & $-354521,1$ & B \\
\hline $353.4-04.5$ & K $6-13$ & $353-042$ & 0,2 & & & 174437,4 & $-364909,5$ & B \\
\hline $353.5-04.9$ & H $1-36$ & $353-041$ & $-119,3$ & & & 174624,1 & $-370035,9$ & B \\
\hline
\end{tabular}

Continua na próxima página... 
Tabela A.1 - Continuação

\begin{tabular}{|c|c|c|c|c|c|c|c|c|}
\hline PN G & Nome & PK & $V_{r}($ hel $)$ & $d_{\mathrm{M} 84}$ & $d_{\mathrm{SSV} 08}$ & $\alpha(1950)$ & $\delta(1950)$ & Amostra \\
\hline $353.7+06.3$ & M $2-7$ & $353+061$ & $-60,2$ & 4,4 & 7,2 & 170201,8 & $-302814,4$ & B \\
\hline $353.7-12.8$ & Wray $16-411$ & $353-121$ & $-50,0$ & & & 182311,2 & $-403141,4$ & B \\
\hline $354.1-04.1$ & Нe $3-1481$ & & $-10,1$ & & & 174441,8 & $-3607 \quad 17,8$ & $\mathrm{~B}$ \\
\hline $354.2+04.3$ & M 2-10 & $354+041$ & $-85,6$ & 3,9 & & 171053,5 & $-311616,3$ & $\mathrm{~A}, \mathrm{~B}$ \\
\hline $354.4+04.0$ & Te 233 & $354+043$ & $-220,0$ & & & 1712 & -3119 & B \\
\hline $354.4-07.8$ & $\mathrm{H} 1-52$ & $354-071$ & $-124,0$ & 4,7 & & 180132,3 & $-373822,1$ & $\mathrm{~B}$ \\
\hline $354.5+03.3$ & Th $3-4$ & $354+031$ & $-165,0$ & & & 171537,7 & $-313600,6$ & $\mathrm{~B}$ \\
\hline $354.6+04.9$ & Te 139 & $354+044$ & 41,0 & & & 170941,1 & $-303634,2$ & $\mathrm{~B}$ \\
\hline $354.7-07.2$ & SB40 & $354-072$ & $-69,0$ & & & 175931,3 & $-370818,4$ & $\mathrm{~B}$ \\
\hline $354.7-10.0$ & SB41 & $354-101$ & 34,0 & & & 181212,2 & $-382856,9$ & $\mathrm{~B}$ \\
\hline $354.9+03.5$ & Th $3-6$ & $355+033$ & $-73,1$ & & & 171606,7 & $-310936,7$ & $\mathrm{~B}$ \\
\hline $355.1+04.7$ & Te 140 & $355+042$ & 64,0 & & & 171151,0 & $-3017 \quad 15,4$ & $\mathrm{~B}$ \\
\hline $355.1-02.9$ & H $1-31$ & $355-024$ & 47,5 & & & 174212,4 & $-343244,8$ & $\mathrm{~B}$ \\
\hline $355.1-06.9$ & M $3-21$ & $355-061$ & $-66,9$ & 4,9 & & 175908,8 & $-363915,0$ & $\mathrm{~B}$ \\
\hline $355.2+03.7$ & Te 137 & $355+031$ & 70,0 & & & 171550,3 & $-305048,9$ & $\mathrm{~B}$ \\
\hline $355.2-02.5$ & H $1-29$ & $355-022$ & $-16,9$ & & & 174054,6 & $-341616,9$ & $\mathrm{~B}$ \\
\hline $355.3-04.0$ & IRAS $17474-3500$ & & $-163,2$ & & & 174726,2 & $-350042,0$ & $\mathrm{~B}$ \\
\hline $355.3-07.5$ & SB42 & $355-071$ & 40,0 & & & 180228,9 & $-364555,7$ & $\mathrm{~B}$ \\
\hline $355.4+02.3$ & Te 138 & $355+024$ & $-46,0$ & & & 172149,3 & $-312559,3$ & $\mathrm{~B}$ \\
\hline $355.4-02.4$ & M $3-14$ & $355-021$ & $-82,3$ & 1,6 & 5,7 & 174101,7 & $-340524,9$ & $\mathrm{~A}, \mathrm{~B}$ \\
\hline $355.4-04.0$ & Hf $2-1$ & $355-041$ & $-84,5$ & 2,4 & 6,2 & 174751,8 & $-345438,5$ & $\mathrm{~B}$ \\
\hline $355.6-02.7$ & H $1-32$ & $355-023$ & $-220,0$ & & & 174247,5 & $-340237,4$ & $\mathrm{~A}, \mathrm{~B}$ \\
\hline $355.7-03.0$ & H $1-33$ & $355-031$ & $-120,0$ & & 12,1 & 174430,4 & $-340705,0$ & $\mathrm{~B}$ \\
\hline $355.7-03.4$ & H $2-23$ & $355-032$ & 82,5 & & & 174538,5 & $-342058,8$ & B \\
\hline $355.7-03.5$ & H $1-35$ & $355-033$ & 123,4 & 2,7 & & 174554,5 & $-342158,6$ & B \\
\hline $355.8-08.7$ & SB43 & $355-081$ & $-74,0$ & & & 180859,8 & $-365340,5$ & $\mathrm{~B}$ \\
\hline $355.9+03.6$ & H $1-9$ & $355+032$ & $-157,6$ & & & 171819,6 & $-301754,5$ & B \\
\hline $355.9-04.2$ & M 1-30 & $355-042$ & $-120,1$ & & & 174939,0 & $-343745,0$ & B \\
\hline $356.0-07.4 \mathrm{~A}$ & SB44 & $356-073$ & 1,0 & & & 180345,5 & $-360309,4$ & $\mathrm{~B}$ \\
\hline $356.0-07.4 \mathrm{~B}$ & SB45 & $356-074$ & $-116,0$ & & & 180330,0 & $-360705,5$ & $\mathrm{~B}$ \\
\hline $356.1+02.7$ & Th $3-13$ & $356+021$ & $-99,0$ & & & 172206,5 & $-303803,9$ & B \\
\hline $356.1-03.3$ & H $2-26$ & $356-031$ & $-156,9$ & 2,2 & & 174632,0 & $-335938,9$ & B \\
\hline $356.1-08.6$ & SB46 & $356-081$ & 54,0 & & & 180916,6 & $-363237,1$ & B \\
\hline $356.2-04.4$ & Cn $2-1$ & $356-041$ & $-169,6$ & 3,6 & 8,0 & 175113,6 & $-342150,1$ & $\mathrm{~A}, \mathrm{~B}$ \\
\hline $356.3-06.2$ & M 3-49 & $356-061$ & $-50,1$ & 4,8 & & 175911,2 & $-351318,5$ & B \\
\hline $356.3-07.3$ & SB47 & $356-075$ & $-27,0$ & & & 180359,6 & $-354608,8$ & $\mathrm{~B}$ \\
\hline $356.4-06.8$ & SB48 & $356-063$ & 10,0 & & & 180153,0 & $-352823,1$ & $\mathrm{~B}$ \\
\hline $356.5+01.5$ & Th $3-55$ & $356+012$ & $-46,2$ & & & 172745,3 & $-305852,1$ & B \\
\hline $356.5+05.1$ & Th $3-3$ & $356+051$ & $-48,0$ & & & 171410,4 & $-285617,3$ & B \\
\hline $356.5-02.3$ & M $1-27$ & $356-022$ & $-47,7$ & & 4,6 & 174328,1 & $-330730,0$ & B \\
\hline $356.5-03.6$ & H $2-27$ & $356-032$ & $-31,3$ & & & 174832,1 & $-334653,2$ & B \\
\hline $356.5-03.9$ & H $1-39$ & $356-033$ & $-85,3$ & & 15,3 & 175002,3 & $-335522,2$ & $\mathrm{~B}$ \\
\hline $356.6-07.8$ & H $1-57$ & $356-072$ & $-20,0$ & 4,4 & & 180627,4 & $-354448,9$ & $\mathrm{~B}$ \\
\hline $356.7-04.8$ & H $1-41$ & $356-042$ & 73,3 & 4,1 & 5,7 & 175400,0 & $-340930,1$ & B \\
\hline $356.7-06.4$ & H $1-51$ & $356-062$ & $-208,0$ & 5,1 & & 180108,8 & $-345813,3$ & $\mathrm{~B}$ \\
\hline
\end{tabular}

Continua na próxima página... 
Tabela A.1 - Continuação

\begin{tabular}{|c|c|c|c|c|c|c|c|c|}
\hline $\mathrm{PN} G$ & Nome & PK & $V_{r}($ hel $)$ & $d_{\mathrm{M} 84}$ & $d_{\mathrm{SSV} 08}$ & $\alpha(1950)$ & $\delta(1950)$ & Amostra \\
\hline $356.8+03.3$ & Th $3-12$ & $356+031$ & 185,4 & & & 172154,6 & $-294237,9$ & B \\
\hline $356.8-05.4$ & H $2-35$ & $356-051$ & $-195,5$ & 3,9 & & 175658,6 & $-342733,3$ & B \\
\hline $356.8-11.7$ & Lo 17 & $356-111$ & $-65,3$ & & & 182425,6 & $-371746,0$ & B \\
\hline $356.9+04.4$ & M 3-38 & $356+042$ & $-171,1$ & 3,6 & 14,1 & 171754,2 & $-290002,9$ & B \\
\hline $356.9+04.5$ & M 2-11 & $356+041$ & 83,7 & 5,1 & 10,8 & 171723,1 & $-285740,6$ & B \\
\hline $356.9-05.8$ & M $2-24$ & $356-052$ & 145,5 & 7,2 & 9,3 & 175843,2 & $-342749,0$ & B \\
\hline $357.0+02.4$ & M 4-4 & $357+025$ & 12,7 & 3,0 & & 172538,1 & $-300522,3$ & B \\
\hline $357.1+01.9$ & Th $3-24$ & $357+027$ & $-197,0$ & & & 172738,9 & $-301458,4$ & B \\
\hline $357.1+03.6$ & M 3-7 & $357+031$ & $-193,6$ & 4,6 & 6,3 & 172123,5 & $-292138,3$ & B \\
\hline $357.1+04.4$ & TeJu 18 & $357+042$ & 52,0 & & & 171827,8 & $-285220,0$ & B \\
\hline $357.1-04.7$ & H $1-43$ & $357-043$ & 49,0 & & 13,3 & 175455,9 & $-334722,6$ & B \\
\hline $357.1-06.1$ & M 3-50 & $357-061$ & 17,5 & 8,3 & & 180045,5 & $-342848,0$ & B \\
\hline $357.2+01.4$ & $\mathrm{Al} 2-\mathrm{H}$ & $357+012$ & $-30,0$ & & & 173004,2 & $-302427,2$ & B \\
\hline $357.2+02.0$ & H $2-13$ & $357+026$ & $-133,7$ & & & 172755,8 & $-300815,1$ & B \\
\hline $357.2+07.4$ & M 4-3 & $357+071$ & 132,5 & & & 170734,6 & $-270503,5$ & $\mathrm{~A}, \mathrm{~B}$ \\
\hline $357.2-04.5$ & H $1-42$ & $357-041$ & $-79,2$ & 4,2 & 6,1 & 175407,0 & $-333524,4$ & B \\
\hline $357.2-09.8$ & SB49 & $357-091$ & 20,0 & & & 181646,7 & $-360841,8$ & B \\
\hline $357.3+03.3$ & M 3-41 & $357+032$ & $-110,0$ & 3,4 & 6,6 & 172248,9 & $-291915,3$ & B \\
\hline $357.3+04.0$ & $\mathrm{H} 2-7$ & $357+041$ & 76,0 & 8,0 & 10,0 & 172014,7 & $-2856 \quad 19,9$ & B \\
\hline $357.3-06.5$ & SB50 & $357-062$ & 0,0 & & & 180248,5 & $-343350,3$ & B \\
\hline $357.4-03.2$ & M $2-16$ & $357-032$ & 90,0 & 1,8 & 7,0 & 174917,6 & $-324511,4$ & $\mathrm{~A}, \mathrm{~B}$ \\
\hline $357.4-03.5$ & M 2-18 & $357-034$ & $-21,1$ & & 15,7 & 175020,7 & $-325812,8$ & B \\
\hline $357.4-04.6$ & M $2-22$ & $357-042$ & $-103,3$ & 2,2 & 8,5 & 175514,6 & $-332823,2$ & B \\
\hline $357.4-07.2$ & SB51 & $357-071$ & $-222,0$ & & & 180556,2 & $-344812,5$ & B \\
\hline $357.5+03.2$ & M 3-42 & $357+034$ & $-267,2$ & 4,0 & 6,9 & 172349,0 & $-291301,1$ & B \\
\hline $357.6+01.0$ & $\operatorname{TrBr} 4$ & $357+013$ & $-122,0$ & & & 173230,8 & $-301933,5$ & B \\
\hline $357.6+01.7$ & H $1-23$ & $357+011$ & $-72,5$ & 0,8 & 9,8 & 172934,9 & $-295809,3$ & $\mathrm{~A}, \mathrm{~B}$ \\
\hline $357.6+02.6$ & H $1-18$ & $357+024$ & $-221,5$ & & & 172631,5 & $-293031,3$ & $\mathrm{~A}, \mathrm{~B}$ \\
\hline $357.6-03.3$ & H $2-29$ & $357-033$ & 107,0 & 1,2 & & 175000,2 & $-3240 \quad 02,0$ & B \\
\hline $357.9-03.8$ & $\mathrm{H} 2-30$ & $358-032$ & $-32,0$ & 4,7 & & 175257,7 & $-323655,9$ & B \\
\hline $357.9-05.1$ & M $1-34$ & $357-051$ & 10,5 & 3,5 & 5,0 & 175804,5 & $-331741,9$ & $\mathrm{~A}, \mathrm{~B}$ \\
\hline $358.0+02.6$ & Th $3-23$ & $358+022$ & 11,7 & & & 172710,7 & $-290756,5$ & B \\
\hline $358.0+07.5$ & TeJu 8 & $358+072$ & 10,1 & & & 170927,4 & $-262151,6$ & B \\
\hline $358.0+09.3$ & Th $3-1$ & $358+091$ & $-17,2$ & & & 170240,0 & $-252100,1$ & B \\
\hline $358.0-02.7$ & $\mathrm{Al} 2-\mathrm{O}$ & $358-024$ & $-78,8$ & & & 174829,7 & $-320220,7$ & B \\
\hline $358.0-05.1$ & Pe $1-11$ & $358-051$ & $-130,6$ & 2,3 & 6,5 & 175825,3 & $-331528,6$ & B \\
\hline $358.2+03.5$ & $\mathrm{H} 2-10$ & $358+032$ & 44,1 & & 13,3 & 172423,2 & $-282838,6$ & B \\
\hline $358.2+03.6$ & M 3-10 & $358+031$ & $-131,3$ & 6,1 & 9,3 & 172410,6 & $-282522,0$ & B \\
\hline $358.2+04.2$ & M $3-8$ & $358+041$ & 76,6 & 4,9 & 7,2 & 172143,2 & $-280314,7$ & B \\
\hline $358.2-01.1$ & Bl D & $358-011$ & $-30,9$ & 1,1 & & 174248,9 & $-310231,3$ & B \\
\hline $358.3+01.2$ & $\mathrm{Bl} \mathrm{B}$ & $358+014$ & $-108,2$ & & & 173348,2 & $-293821,5$ & B \\
\hline $358.3+03.0$ & $\mathrm{H} 1-17$ & $358+037$ & $-29,7$ & & 25,7 & 172630,6 & $-283803,0$ & $\mathrm{~A}, \mathrm{~B}$ \\
\hline $358.3-07.3$ & SB52 & $358-072$ & $-49,0$ & & & 180821,1 & $-340105,7$ & B \\
\hline $358.3-21.6$ & IC 1297 & $358-211$ & 10,3 & 3,0 & 4,9 & 191357,3 & $-394211,4$ & $\mathrm{~A}, \mathrm{~B}$ \\
\hline $358.4+03.3$ & Th $3-19$ & $358+033$ & 122,0 & & 18,2 & 172532,2 & $-282456,0$ & B \\
\hline
\end{tabular}

Continua na próxima página... 
Tabela A.1 - Continuação

\begin{tabular}{|c|c|c|c|c|c|c|c|c|}
\hline $\mathrm{PN} \mathrm{G}$ & Nome & PK & $V_{r}($ hel $)$ & $d_{\mathrm{M} 84}$ & $d_{\mathrm{SSV} 08}$ & $\alpha(1950)$ & $\delta(1950)$ & Amostra \\
\hline $358.5+03.7$ & $\mathrm{Al} 2-\mathrm{B}$ & $358+039$ & $-158,0$ & & & 172437,9 & $-280833,5$ & B \\
\hline $358.5+05.4$ & M 3-39 & $358+051$ & 1,5 & 1,5 & 2,1 & 171804,0 & $-270842,5$ & $\mathrm{~B}$ \\
\hline $358.5-02.5$ & M 4-7 & $358-021$ & $-233,8$ & 2,6 & 6,4 & 174829,8 & $-313517,0$ & B \\
\hline $358.5-04.2$ & H $1-46$ & $358-041$ & $-44,3$ & 4,3 & & 175546,4 & $-322132,3$ & $\mathrm{~B}$ \\
\hline $358.5-07.3$ & NGC 6563 & $358-071$ & $-29,5$ & 1,9 & 1,6 & 180844,1 & $-335252,6$ & $\mathrm{~A}, \mathrm{~B}$ \\
\hline $358.6+01.8$ & M 4- 6 & $358+011$ & $-272,9$ & & & 173203,4 & $-290115,6$ & B \\
\hline $358.6+07.8$ & M 3-36 & $358+071$ & 1,4 & 5,9 & 14,3 & 170934,0 & $-254005,3$ & B \\
\hline $358.6-05.5$ & M 3-51 & $358-054$ & 31,8 & 4,6 & & 180139,2 & $-325415,6$ & $\mathrm{~B}$ \\
\hline $358.7+05.2$ & M 3-40 & $358+052$ & 32,8 & 4,1 & 11,6 & 171920,8 & $-270552,2$ & B \\
\hline $358.7-02.7$ & $\mathrm{Al} 2-\mathrm{R}$ & $358-025$ & $-119,0$ & & & 175021,9 & $-312450,6$ & B \\
\hline $358.7-05.1$ & SB53 & $358-055$ & $-14,0$ & & & 180012,0 & $-323734,5$ & B \\
\hline $358.7-05.2$ & H $1-50$ & $358-053$ & 70,3 & & & 180036,8 & $-324152,0$ & $\mathrm{~B}$ \\
\hline $358.8+03.0$ & Th $3-26$ & $358+038$ & 197,4 & & 7,5 & 172760,0 & $-281237,8$ & B \\
\hline $358.8+04.0$ & Th $3-15$ & $358+042$ & 33,0 & & & 172402,2 & $-274129,5$ & $\mathrm{~B}$ \\
\hline $358.8+04.1$ & SaWe 2 & $358+043$ & 46,0 & & & 172351,8 & $-273804,5$ & B \\
\hline $358.9+03.2$ & H $1-20$ & $358+036$ & 189,5 & 4,1 & 7,5 & 172734,8 & $-280152,3$ & B \\
\hline $358.9+03.4$ & H $1-19$ & $358+034$ & 23,4 & & & 172653,6 & $-275700,1$ & $\mathrm{~B}$ \\
\hline $358.9-00.7$ & M $1-26$ & $358-002$ & $-7,2$ & 1,4 & & 174245,1 & $-301052,2$ & $\mathrm{~A}, \mathrm{~B}$ \\
\hline $358.9-03.7$ & $\mathrm{H} 1-44$ & $358-031$ & 97,1 & 3,2 & & 175455,6 & $-314241,0$ & B \\
\hline $359.0+02.8$ & $\mathrm{Al} 2-\mathrm{G}$ & $359+025$ & $-262,0$ & & & 172913,3 & $-281219,9$ & B \\
\hline $359.0-04.1$ & M 3-48 & $359-041$ & $-12,0$ & 4,4 & & 175641,4 & $-315420,1$ & $\mathrm{~B}$ \\
\hline $359.0-04.8$ & M 2-25 & $359-043$ & 10,6 & 2,0 & 2,2 & 175930,9 & $-320935,0$ & $\mathrm{~B}$ \\
\hline $359.1+15.1$ & A 40 & $359+151$ & 2,0 & 4,7 & 3,9 & 164536,4 & $-205537,1$ & B \\
\hline $359.1-01.7$ & M 1-29 & $359-011$ & $-41,0$ & 0,7 & 4,1 & 174704,8 & $-303405,4$ & $\mathrm{~B}$ \\
\hline $359.1-02.3$ & M 3-16 & $359-022$ & 63,4 & 1,4 & 6,7 & 174932,4 & $-304855,7$ & B \\
\hline $359.1-02.9$ & M $3-46$ & $359-024$ & $-71,0$ & 2,6 & & 175151,6 & $-311147,5$ & B \\
\hline $359.2+01.2$ & 19W32 & $359+013$ & 55,3 & & & 173552,4 & $-285457,2$ & $\mathrm{~B}$ \\
\hline $359.2+04.7$ & Th $3-14$ & $359+041$ & $-239,2$ & & & 172236,8 & $-265511,8$ & B \\
\hline $359.3+01.4$ & Th $3-35$ & $359+011$ & 54,5 & & & 173532,0 & $-284105,4$ & B \\
\hline $359.3+03.6$ & $\mathrm{Al} 2-\mathrm{E}$ & $359+035$ & $-58,6$ & & & 172706,2 & $-272802,8$ & $\mathrm{~B}$ \\
\hline $359.3-00.9$ & $\mathrm{Hb} 5$ & $359-001$ & $-24,0$ & 1,2 & 1,7 & 174443,9 & $-295842,2$ & $\mathrm{~A}, \mathrm{~B}$ \\
\hline $359.3-01.8$ & M 3-44 & $359-012$ & $-99,2$ & 1,0 & & 174806,0 & $-302308,0$ & B \\
\hline $359.3-03.1$ & M 3-17 & $359-031$ & $-32,6$ & & 11,6 & 175311,6 & $-310354,1$ & B \\
\hline $359.3-06.0$ & SB54 & $359-062$ & 38,0 & & & 180515,0 & $-323019,9$ & B \\
\hline $359.4+02.3$ & Th $3-32$ & $359+024$ & $-167,0$ & & & 173206,4 & $-280506,3$ & $\mathrm{~B}$ \\
\hline $359.4-03.4$ & H $2-33$ & $359-032$ & 107,0 & 3,5 & 7,7 & 175458,4 & $-310751,1$ & B \\
\hline $359.4-08.5$ & SB55 & $359-081$ & 163,0 & & & 181608,2 & $-333822,6$ & B \\
\hline $359.6+02.2$ & Al 2-I & $359+027$ & 97,5 & & & 173305,1 & $-275855,7$ & B \\
\hline $359.6-04.8$ & H $2-36$ & $359-044$ & $-119,7$ & & & 180053,0 & $-313924,7$ & B \\
\hline $359.7-01.8$ & M 3-45 & $359-013$ & 28,3 & 1,0 & & 174853,5 & $-300432,2$ & B \\
\hline $359.7-02.6$ & H $1-40$ & $359-023$ & 64,0 & & 7,2 & 175222,8 & $-303306,0$ & B \\
\hline $359.7-04.4$ & KFL 3 & $359-04$ & 204,6 & & & 175938,4 & $-312404,0$ & B \\
\hline $359.8+01.5$ & K 6-6 & $359+014$ & $-161,0$ & & & 173608,8 & $-281331,2$ & B \\
\hline $359.8+02.4$ & Th $3-33$ & $359+023$ & 72,0 & & & 173239,5 & $-274127,9$ & B \\
\hline $359.8+03.7$ & Th $3-25$ & $359+032$ & $-92,7$ & & 14,5 & 172739,2 & $-270343,8$ & B \\
\hline
\end{tabular}

Continua na próxima página... 
Tabela A.1 - Continuação

\begin{tabular}{|c|c|c|c|c|c|c|c|c|}
\hline $\mathrm{PN} \mathrm{G}$ & Nome & PK & $V_{r}($ hel $)$ & $d_{\mathrm{M} 84}$ & $d_{\mathrm{SSV} 08}$ & $\alpha(1950)$ & $\delta(1950)$ & Amostra \\
\hline $359.8+05.2$ & TeJu 19 & $359+053$ & $-34,4$ & & & 172217,4 & $-260916,3$ & B \\
\hline $359.8+05.6$ & M 2-12 & $359+051$ & 63,4 & 5,7 & & 172055,6 & $-255639,9$ & $\mathrm{~B}$ \\
\hline $359.8+06.9$ & M 3-37 & $359+061$ & $-74,2$ & 9,8 & 16,6 & 171608,6 & $-2514 \quad 13,5$ & $\mathrm{~B}$ \\
\hline $359.8-07.2$ & M $2-32$ & $359-071$ & $-54,3$ & & & 181134,1 & $-323752,9$ & $\mathrm{~B}$ \\
\hline $359.9+05.1$ & M 3-9 & $359+052$ & $-83,9$ & 3,4 & 3,3 & 172237,1 & $-260919,4$ & $\mathrm{~B}$ \\
\hline $359.9-04.5$ & M $2-27$ & $359-042$ & 144,8 & & 12,4 & 180038,2 & $-311756,6$ & $\mathrm{~A}, \mathrm{~B}$ \\
\hline $359.9-05.4$ & KFL 9 & $359-05$ & 0,0 & & & 180404,3 & $-314321,9$ & $\mathrm{~B}$ \\
\hline $359.9-07.4$ & SB56 & $359-072$ & 222,0 & & & $1812 \quad 16,0$ & $-323901,1$ & B \\
\hline
\end{tabular}


Apêndice B

\section{NPs com Determinações do Movimento Próprio}

A tabela $\mathbb{B} . \mathbb{l}$ apresenta as nebulosas planetárias de ambas as amostras com determinações do movimento próprio retiradas da base de dados SIMBAD. As três primeiras colunas $(1,2,3)$ mostram a identificação PN G (Acker et al, 19.92), nome usual e identificação PK (Perek e Kohoutek, 1967) das nebulosas planetárias, respectivamente. A quarta

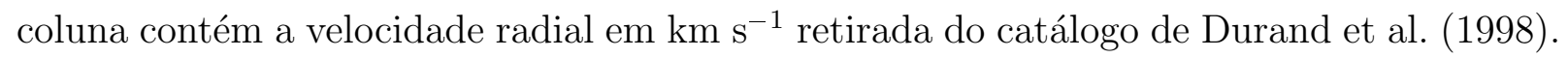
A quinta e a sexta colunas apresentam as distâncias em kpc do catálogo de Maciel (1984) e de Stanghellini et al. (20108), respectivamente. A sétima e a oitava colunas apresentam os dados do movimento próprio em ascensão reta e em declinação em milisegundos de arco por ano. A última coluna (9) designa qual amostra cada uma pertence.

Tabela B.1 - Amostra de nebulosas planetárias com determinações do movimento próprio.

\begin{tabular}{ccccccccc}
\hline \hline PN G & Nome & PK & $V_{r}$ (hel) & $d_{\text {M } 84}$ & $d_{\text {SSV08 }}$ & $\mu_{\alpha}$ & $\mu_{\delta}$ & Amostra \\
\hline $001.5-06.7$ & SwSt 1 & $001-062$ & $-17,0$ & 2,1 & & $-26,07$ & $-13,63$ & A, B \\
$010.1+00.7$ & NGC 6537 & $010+001$ & $-16,9$ & 0,9 & & $+17,50$ & $-1,40$ & A, B \\
$011.0+05.8$ & NGC 6439 & $011+051$ & $-96,7$ & 3,8 & 6,3 & $-29,80$ & 7,20 & A, B \\
$011.7-06.6$ & M 1-55 & $011-061$ & $-22,4$ & & & $-6,90$ & $+31,90$ & B \\
$017.6-10.2$ & A 51 & $017-101$ & 16,8 & 2,7 & 1,5 & $+0,80$ & $-19,30$ & B \\
$025.3+40.8$ & IC 4593 & $025+401$ & 22,0 & 2,4 & 3,2 & $-8,70$ & $+4,10$ & A, B \\
$034.6+11.8$ & NGC 6572 & $034+111$ & $-10,1$ & 0,8 & 1,7 & $+8,90$ & $-4,00$ & A, B \\
$036.1-57.1$ & NGC 7293 & $036-571$ & $-28,2$ & 0,2 & 0,2 & $+32,00$ & $-5,00$ & A, B \\
$038.2+12.0$ & Cn 3-1 & $038+121$ & $-11,2$ & 2,9 & 6,0 & $-4,60$ & $-9,40$ & A, B \\
$043.1+37.7$ & NGC 6210 & $043+371$ & $-36,2$ & 1,3 & 2,3 & $-9,60$ & $-16,00$ & A, B \\
$051.4+09.6$ & Hu 2-1 & $051+091$ & 14,0 & 1,9 & & $+10,04$ & $-0,71$ & A, B \\
$054.1-12.1$ & NGC 6891 & $054-121$ & 42,3 & 2,1 & 3,6 & 2,00 & $-2,80$ & A, B \\
$058.3-10.9$ & IC 4997 & $058-101$ & $-66,2$ & 2,3 & & $-3,60$ & $-3,80$ & A, B \\
$060.3-07.3$ & He 1-5 & $060-071$ & 38,9 & 2,8 & 3,1 & $+0,44$ & $-8,60$ & B \\
$064.7+05.0$ & BD+30 3639 & $064+051$ & $-31,4$ & 0,6 & 3,0 & $-4,08$ & $-9,21$ & A, B \\
$083.5+12.7$ & NGC 6826 & $083+121$ & $-6,2$ & 0,7 & 1,6 & $-11,00$ & $-9,70$ & A, B \\
\hline
\end{tabular}

Continua na próxima página... 
Tabela B.1 - Continuação

\begin{tabular}{|c|c|c|c|c|c|c|c|c|}
\hline $\mathrm{PN} \mathrm{G}$ & Nome & $\mathrm{PK}$ & $V_{r}(\mathrm{hel})$ & $d_{\mathrm{M} 84}$ & $d_{\text {SSV08 }}$ & $\mu_{\alpha}$ & $\mu_{\delta}$ & Amostra \\
\hline $084.9-03.4$ & NGC 7027 & $084-031$ & 8,8 & 0,7 & & $+6,00$ & $-16,00$ & A, B \\
\hline $096.4+29.9$ & NGC 6543 & $096+291$ & $-66,1$ & 0,7 & 1,6 & $+3,60$ & $+6,00$ & A, B \\
\hline $100.0-08.7$ & Me 2-2 & $100-081$ & $-152,0$ & & & $-4,00$ & $-2,10$ & A, B \\
\hline $106.5-17.6$ & NGC 7662 & $106-171$ & $-13,2$ & 0,8 & 2,0 & $+12,20$ & $-1,00$ & A, B \\
\hline $118.8-74.7$ & NGC 246 & $118-741$ & $-46,0$ & 0,4 & 0,5 & $-23,85$ & $-4,89$ & A, B \\
\hline $120.0+09.8$ & NGC 40 & $120+091$ & $-20,4$ & 0,8 & 1,2 & $-10,66$ & $+5,25$ & A, B \\
\hline $123.6+34.5$ & IC 3568 & $123+341$ & $-41,1$ & 2,1 & 2,7 & $+0,80$ & $-24,00$ & A, B \\
\hline $165.5-15.2$ & NGC 1514 & $165-151$ & 59,8 & 0,8 & 0,8 & $-8,01$ & $+2,60$ & A, B \\
\hline $197.8+17.3$ & NGC 2392 & $197+171$ & 75,0 & 1,1 & 1,3 & $-3,97$ & $+1,59$ & A, B \\
\hline $211.2-03.5$ & M 1-6 & $211-031$ & 65,6 & 3,0 & & $-2,30$ & $-6,10$ & A, B \\
\hline $215.2-24.2$ & IC 418 & $215-241$ & 61,9 & 0,8 & & $-1,20$ & $+2,50$ & A, B \\
\hline $215.6+03.6$ & NGC 2346 & $215+031$ & 21,8 & 1,5 & 1,4 & $-3,14$ & $-0,47$ & A, B \\
\hline $220.3-53.9$ & NGC 1360 & $220-531$ & 41,8 & 0,5 & 0,4 & $+1,77$ & $+24,91$ & B \\
\hline $253.9+05.7$ & M 3-6 & $254+051$ & 45,8 & 2,6 & 4,2 & $+2,00$ & $+2,00$ & A, B \\
\hline $261.0+32.0$ & NGC 3242 & $261+321$ & 4,4 & 0,8 & 1,1 & $-17,10$ & $+4,00$ & A, B \\
\hline $272.1+12.3$ & NGC 3132 & $272+121$ & $-16,0$ & 1,1 & 1,3 & $-9,00$ & $+5,70$ & A, B \\
\hline $279.6-03.1$ & He $2-36$ & $279-031$ & $-7,1$ & 2,7 & 2,4 & $-5,72$ & $+6,38$ & B \\
\hline $300.7-02.0$ & Sa $2-86$ & $300-021$ & $-7,5$ & 2,6 & & $-4,80$ & $-10,20$ & A, B \\
\hline $303.6+40.0$ & A 35 & $303+401$ & $-6,6$ & 0,5 & 0,2 & $-60,91$ & $-13,41$ & B \\
\hline $309.0+00.8$ & He $2-96$ & $309+001$ & $-53,2$ & 0,9 & & $+3,40$ & $+6,20$ & B \\
\hline $310.3+24.7$ & Lo 8 & $310+241$ & $-3,0$ & & & $-6,70$ & $+3,30$ & B \\
\hline $315.1-13.0$ & He $2-131$ & $315-131$ & $-1,2$ & 1,6 & & $-7,90$ & $-2,00$ & A, B \\
\hline $318.4+41.4$ & A 36 & $318+411$ & 36,8 & 0,6 & 0,4 & $+17,06$ & $+4,62$ & B \\
\hline $320.1-09.6$ & He $2-138$ & $320-091$ & $-56,9$ & 3,1 & 4,8 & $-6,17$ & $-6,09$ & A, B \\
\hline $325.8-12.8$ & He $2-182$ & $325-121$ & $-89,5$ & 3,8 & & $+3,10$ & $-6,90$ & B \\
\hline $327.8+10.0$ & NGC 5882 & $327+101$ & 9,7 & 1,6 & 2,4 & $-9,40$ & $-1,80$ & A, B \\
\hline $330.7+04.1$ & Cn $1-1$ & $330+041$ & $-9,0$ & & & $-3,89$ & $-0,76$ & B \\
\hline $331.1-05.7$ & PC 11 & $331-051$ & $-52,0$ & 6,2 & & $-5,10$ & $-18,20$ & B \\
\hline $331.3-12.1$ & Не $3-1357$ & $331-121$ & 13,0 & & & $-0,80$ & $-4,00$ & B \\
\hline $332.9-09.9$ & Не $3-1333$ & $332-091$ & $-65,6$ & & & $-69,82$ & $-35,71$ & B \\
\hline $334.8-07.4$ & SaSt $2-12$ & $334-071$ & $-63,0$ & & & $-5,44$ & $-6,24$ & B \\
\hline $345.2-08.8$ & Tc 1 & $345-081$ & $-84,1$ & 1,0 & 2,7 & $-1,60$ & $-11,40$ & A, B \\
\hline $349.3-04.2$ & Lo 16 & $349-041$ & 13,0 & & & $-6,00$ & $-4,30$ & B \\
\hline $352.9-07.5$ & $\mathrm{Fg} 3$ & $352-071$ & 4,0 & 2,0 & & $+4,20$ & $-9,80$ & B \\
\hline
\end{tabular}


Apêndice C

\section{Artigo}

Os principais resultados dessa dissertação estão apresentados no artigo Kinematic ages of the central stars of planetary nebulae de Maciel, Rodrigues e Costa (2010a), publicado na Revista Mexicana de Astronomia y Astrofisica em outubro de 2011. 


\title{
KINEMATIC AGES OF THE CENTRAL STARS OF PLANETARY NEBULAE
}

\author{
W. J. Maciel, T. S. Rodrigues, and R. D. D. Costa \\ Instituto de Astronomia, Geofísica e Ciências Atmosféricas \\ Universidade de São Paulo, Brazil
}

Received 2011 July 01; accepted 2011 August 11

\section{RESUMEN}

Se determina la distribución de edades de las estrellas centrales de nebulosas planetarias (CSPN) utilizando dos métodos basados en sus propiedades cinemáticas. En primer lugar, se comparan las velocidades de rotación de las nebulosas esperadas a partir de sus distancias galactocéntricas con los valores predichos por la curva de rotación, y las diferencias encontradas se atribuyen a las distintas edades de las estrellas evolucionadas. Se usa la relación entre las edades y la dispersión de velocidades del relevamiento Geneva-Copenhagen para obtener la distribución de edades. En segundo lugar, se determinan las componentes $U, V, W$ de las velocidades de las estrellas, y se usan las relaciones edad-dispersión de velocidades para inferir la distribución de edades. Hemos aplicado estos métodos a dos muestras de nebulosas planetarias en nuestra galaxia. Los resultados son similares para ambas, y muestran que la distribución de edades de las CSPN está concentrada en edades menores que 5 giga-años, y que tiene un pico entre 1 y 3 giga-años.
\end{abstract}

\section{ABSTRACT}

The age distribution of the central stars of planetary nebulae (CSPN) is estimated using two methods based on their kinematic properties. First, the expected rotation velocities of the nebulae at their Galactocentric distances are compared with the predicted values from the rotation curve, and the differences are attributed to the different ages of the evolved stars. Adopting the relation between the ages and the velocity dispersions determined by the Geneva-Copenhagen survey, the age distribution can be derived. Second, the $U, V, W$, velocity components of the stars are determined, and the corresponding age-velocity dispersion relations are used to infer the age distribution. These methods have been applied to two samples of PN in the Galaxy. The results are similar for both samples, and show that the age distribution of the PN central stars concentrates at ages lower than 5 Gyr, peaking at about 1 to 3 Gyr.

Key Words: planetary nebulae: general — stars: AGB and Post-AGB — stars: fundamental parameters - stars: general

\section{INTRODUCTION}

Planetary nebulae $(\mathrm{PN})$ are evolved objects ejected by stars with main sequence masses in the range of 0.8 and $8 M_{\odot}$, so that the expected ages of their central stars are of the order of, or greater than, about 1 Gyr. However, the relatively large mass bracket of their progenitor stars implies that an age distribution is to be expected, which has some consequences for the interpretation of the PN data in the Galaxy and other stellar systems. The de- termination of ages of the central stars is a difficult problem, and most usual methods have large uncertainties when applied to intermediate and old age objects. We have recently developed three different methods to estimate the age distribution of the CSPN (Maciel, Costa, \& Idiart 2010, see also Maciel, Costa, \& Uchida 2003; Maciel, Lago, \& Costa 2005, 2006), and have applied these methods to a sample of PN in the disk of the Galaxy, most of which are located in the solar neighborhood, within 
$3 \mathrm{kpc}$ of the Sun. These methods include the determination of the age distribution of CSPN using (i) an age-metallicity relation that also depends on the Galactocentric distance, (ii) an age-metallicity relation obtained for the disk, and (iii) the central star masses obtained from the observed nitrogen abundances. We concluded that most CSPN in our sample have ages under $6 \mathrm{Gyr}$, and that the age distribution is peaked around 2-4 Gyr. The average uncertainties were estimated as 1-2 Gyr, and the results were compared with the expected distribution based both on the observed mass distribution of white dwarfs and on the age distribution derived from available masses of CSPN.

In the present work we develop two additional and more accurate methods to estimate the age distribution of the CSPN based on their kinematical properties, namely: (i) a method based on the expected rotation velocities of the nebulae at their Galactocentric distances, which are then compared with the predicted values for a given rotation curve, the differences being attributed to the different ages of the evolved stars; (ii) a method based on the derived $U, V, W$, velocity components of the stars and their corresponding dispersions. In both cases, the age-velocity dispersion relations from the GenevaCopenhagen survey are used to infer the age distribution. These methods are applied to two PN samples, (i) the previous sample of disk PN used by Maciel et al. (2010), for which a detailed data set is available, and (ii) a sample containing all PN for which accurate radial velocities are known. The methods are developed in $\S 2$, and the samples used are described in $\S 3$. The main results and discussion are given in $\S 4$.

\section{DETERMINATION OF THE AGE DISTRIBUTION OF CSPN}

\subsection{Method 1: The PN rotation velocity}

As objects of intermediate age, $\mathrm{PN}$ in the disk of the Galaxy describe a rotation curve similar to the one defined by younger objects, such as HII regions, although with a higher dispersion, as discussed in detail by Maciel \& Lago (2005). Therefore, the discrepancies between the rotation velocities inferred from the $\mathrm{PN}$ radial velocities and distances and the velocities expected from the known rotation curve may be at least partially ascribed to their evolved status. In other words, a given nebula located at a distance $d$, with galactic coordinates $\ell$ and $b$ and observed heliocentric radial velocity $V_{r}($ hel $)$ can be associated with a rotation velocity $\theta(R)$, after obtaining its Galactocentric distance $R$ and its radial velocity relative to the Local Standard of Rest (LSR), $V_{r}$ (LSR). Assuming circular orbits, the rotation velocity $\theta(R)$ at the Galactocentric distance $R$ can be written as

$$
\theta(R)=\frac{R}{R_{o}}\left[\frac{V_{r}(\mathrm{LSR})}{\sin \ell \cos b}+\theta_{0}\right],
$$

where $R_{0}$ and $\theta_{0}$ are the Galactocentric distance and rotation velocity at the solar position (see for example Maciel \& Lago 2005; Maciel \& Dutra 1992). On the other hand, the expected rotation velocity at the given Galactocentric distance, $\theta_{c}(R)$, can be obtained from an adopted rotation curve. The difference $\Delta \theta=\left|\theta(R)-\theta_{c}(R)\right|$ can then be considered as proportional to the age difference between the $\mathrm{PN}$ and the objects defining the rotation curve. We have adopted the radial velocities from the catalogue by Durand, Acker, \& Zijlstra (1998), and two distance scales, those by Maciel (1984) and Stanghellini, Shaw, \& Villaver (2008). The first one was based on a relationship between the ionized mass and the radius of the nebulae, while the second is an update of the distance scale by Cahn, Kaler, \& Stanghellini (1992), using a modified Shklovksy method following Daub (1982). Since the distances of planetary nebulae in the Galaxy may contain large individual uncertainties, the use of two different scales which are considered as "short" (Maciel 1984) and "long" (Stanghellini et al. 2008) ensures that these uncertainties will not affect the derived age distributions. We have adopted $R_{0}=8.0 \mathrm{kpc}$ for the distance of the Sun to the centre and $\theta_{0}=220 \mathrm{~km} \mathrm{~s}^{-1}$ for the rotation velocity at $R_{0}$. Slightly different values can be found in the literature (see for example Perryman 2009, and Reid 2010), but the values above are frequently adopted, so that a comparison with other work is made easier. For the "theoretical" rotation curve we have also adopted two possibilities, namely, the PN curve derived by Maciel \& Lago (2005), and the HII region curve derived by Clemens (1985). In the first case, the rotation velocity can be written as

$$
\theta_{c}(R)=a_{0}+a_{1} R+a_{2} R^{2},
$$

where the constants are $a_{0}=269.2549, a_{1}=$ -14.7321 , and $a_{2}=0.7847$, the Galactocentric distance $R$ is given in $\mathrm{kpc}$ and $\theta_{c}(R)$ in $\mathrm{km} \mathrm{s}^{-1}$. For the CO/HII region based Clemens (1985) curve, we have made an adjustment for $R_{0}=8.0 \mathrm{kpc}$ and $\theta_{0}(R)=220 \mathrm{~km} \mathrm{~s}^{-1}$, in which case we have

$$
\theta_{c}(R)=\sum a_{i} R^{i}
$$

where the constants are given in Table 1 , with the same units as in equation (2). 
TABLE 1

COEFFICIENTS OF THE POLYNOMIAL GIVEN BY EQUATION (3)

\begin{tabular}{cccccc}
\hline$R(\mathrm{kpc})$ & $0-0.765$ & $0.765-2.9$ & $2.9-3.825$ & $3.825-13$ & $>13$ \\
\hline$a_{0}$ & 0.0 & 325.0912 & 329.8 & -2346.0 & 230.6 \\
$a_{1}$ & 3069.81 & -248.1467 & -250.1 & 2507.60391 & $\ldots$ \\
$a_{2}$ & -15809.8 & 231.87099 & 231.87099 & -1024.068760 & $\ldots$ \\
$a_{3}$ & 43980.1 & -110.73531 & -110.73531 & 224.562732 & $\ldots$ \\
$a_{4}$ & -68287.3 & 25.073006 & 25.073006 & -28.4080026 & $\ldots$ \\
$a_{5}$ & 54904.0 & -2.110625 & -2.110625 & 2.0697271 & $\ldots$ \\
$a_{6}$ & -17731.0 & $\ldots$ & $\ldots$ & -0.080508084 & $\ldots$ \\
$a_{7}$ & $\ldots$ & $\ldots$ & $\ldots$ & 0.00129348 & $\ldots$ \\
\hline
\end{tabular}

TABLE 2

COEFFICIENTS OF EQUATION

\begin{tabular}{ccc}
\hline & $a$ & $b$ \\
\hline$U$ & 0.39 & 1.31 \\
$V$ & 0.40 & 1.10 \\
$W$ & 0.53 & 0.94 \\
Total & 0.40 & 1.40 \\
\hline
\end{tabular}

The recent Geneva-Copenhagen Survey of the Solar Neighborhood (cf. Nordström et al. 2004; Holmberg, Nordström, \& Andersen 2007, 2009) has considerably improved the relations involving the ages, kinematics, and chemical composition of a large sample containing about $14000 \mathrm{~F}$ and G nearby stars. Using basically the original Hipparcos parallaxes, uvby $-\beta$ photometry and the Padova stellar evolution models, several basic relations were investigated. In particular, high correlations have been obtained between the velocity dispersions $\sigma_{U}, \sigma_{V}$, $\sigma_{W}$, and $\sigma_{T}$ and the ages of the stars, which clearly show a smooth increase of the velocity dispersions (of the $U, V, W$ components and the total velocity $T)$ with time. From the calibration by Holmberg et al. (2009) these correlations can be approximately written as

$$
\log \sigma=a \log t+b,
$$

where the age $t$ is expressed in Gyr and the constants $a, b$ are given in Table 2. This approximation is valid in the age interval $0<t(\mathrm{Gyr})<14$ with an estimated average uncertainty of about $25 \%$. Method 1 consists of assuming that the discrepancy in the rotation velocity $\Delta \theta$ is due to the evolved status of the CSPN, so that we should expect a correlation
TABLE 3

\section{PARAMETERS FOR METHOD 1}

\begin{tabular}{cccc}
\hline Distance & Rotation Curve & Dispersion & Age \\
\hline Maciel & PN & $\sigma_{V}$ & $t_{1}$ \\
Maciel & PN & $\sigma_{T}$ & $t_{2}$ \\
Maciel & Clemens & $\sigma_{V}$ & $t_{3}$ \\
Maciel & Clemens & $\sigma_{T}$ & $t_{4}$ \\
Stanghellini & PN & $\sigma_{V}$ & $t_{5}$ \\
Stanghellini & PN & $\sigma_{T}$ & $t_{6}$ \\
Stanghellini & Clemens & $\sigma_{V}$ & $t_{7}$ \\
Stanghellini & Clemens & $\sigma_{T}$ & $t_{8}$ \\
\hline
\end{tabular}

between $\Delta \theta$ and the velocity dispersion, as given by equation (4). Since in this method we are using the rotation velocity, we have considered two possibilities, according to which the velocity discrepancy $\Delta \theta$ can be associated with (i) the $V$ component of the total velocity $\left(\sigma_{V}\right)$, or (ii) the total velocity $\left(\sigma_{T}\right)$. Moreover, since we are adopting two distance scales and two theoretical rotation curves, we have 8 different age distributions for Method 1, characterized by the timescales $t_{1}$ to $t_{8}$, as explained in Table 3 .

\subsection{Method 2: The $U, V, W$ velocity components}

Method 2 is also a kinematic method, and in principle more accurate than Method 1, as discussed in more detail in $\S 4$. From the PN radial velocities and distances, we have estimated their proper motions both in right ascension and declination, $\mu_{\alpha}$ and $\mu_{\delta}$. We have assumed that, on average, the tangential velocities are similar to the radial velocities, namely $V_{t} \simeq V_{r}$. In view of the large distances of the nebulae, this hypothesis in practice does not introduce any major uncertainties in the 
TABLE 4

PARAMETERS FOR METHOD 2

\begin{tabular}{ccc}
\hline Distance & Dispersion & Age \\
\hline Maciel & $\sigma_{U}$ & $t_{9}$ \\
Maciel & $\sigma_{V}$ & $t_{10}$ \\
Maciel & $\sigma_{W}$ & $t_{11}$ \\
Maciel & $\sigma_{T}$ & $t_{12}$ \\
Stanghellini & $\sigma_{U}$ & $t_{13}$ \\
Stanghellini & $\sigma_{V}$ & $t_{14}$ \\
Stanghellini & $\sigma_{W}$ & $t_{15}$ \\
Stanghellini & $\sigma_{T}$ & $t_{16}$ \\
\hline
\end{tabular}

results. Considering further the equatorial coordinates $(\alpha, \delta)$ of the $\mathrm{PN}$, we have used the equations by Boesgaard \& Tripicco (1986) to derive the $U$, $V, W$ velocity components of the nebulae, as well as the total velocity $T$ and the velocity dispersions $\sigma_{U}, \sigma_{V}, \sigma_{W}$, and $\sigma_{T}$. According to these equations we derive the following parameters: $C=f(d)$, $X=f\left(C, \mu_{\alpha}, \mu_{\delta}, \alpha, \delta, V_{r}\right), Y=f\left(C, \mu_{\alpha}, \mu_{\delta}, \alpha, \delta, V_{r}\right)$, and $Z=f\left(C, \mu_{\delta}, \delta, V_{r}\right)$, from which the velocities can be written as $U=f(X, Y, Z), V=f(X, Y, Z)$, $W=f(X, Y, Z)$, and $T=f(X, Y, Z)$, so that the dispersions are given by

$$
\sigma_{i}=\sqrt{\left(V_{i}-\bar{V}_{i}\right)^{2}},
$$

where $V_{i}$ stands for the velocities $U, V, W, T$. Then, we have again used the detailed correlations between the velocity dispersions and the ages as given by the Geneva-Copenhagen survey (Holmberg et al. 2009), adopting the same coefficients given in Table 2. We have used the same distance scales (Maciel 1984; Stanghellini et al. 2008), so that we have again 8 different age distributions, corresponding to the timescales $t_{9}$ to $t_{16}$, as described in Table 4 .

In practice, we have considered several additional cases, in order to better investigate the hypothesis of $V_{t} \simeq V_{r}$. Assuming that these velocities are of the same magnitude, but allowing for the possibility of different signs, we have as a result several possibilities for the proper motions $\mu_{\alpha}$ and $\mu_{\delta}$, all of which are consistent with either $V_{t} \simeq V_{r}$ or $\left|V_{t}\right| \simeq\left|V_{r}\right|$. It turns out that these possibilities produce very similar age distributions, which will be discussed in $\S 4$. Therefore, we will present only the distributions of the ages $t_{9}$ to $t_{16}$, as defined in Table 4 , for the cases where $\mu_{\alpha} \simeq \mu_{\delta} \simeq 0$.

An interesting alternative to overcome the lack of proper motion and tangential velocity measure- ments would be to apply the singular value decomposition (SVD) technique, as used by Branham (2010) to solve the inverse problem, that is, obtaining the space velocities from available proper motions. However, in view of the similarity of the results for different assumptions regarding the tangential velocities, it is unlikely that this technique would produce very different results than presented here.

\section{THE SAMPLES}

As mentioned in the Introduction, we have considered two samples of Milky Way PN. In order to make comparisons with our previous work, we have first considered the same sample used by Maciel et al. (2003, 2005, 2006), which we will call Sample 1. This sample contains 234 well-observed nebulae located in the solar neighborhood and in the disk, for which all data were obtained with the highest accuracy. Their Galactocentric distances are in the range $4<R(\mathrm{kpc})<14$, and most $(69 \%)$ are located in the solar neighborhood, with distances $d<3 \mathrm{kpc}$.

The second sample considered in this work, called Sample 2, includes all the nebulae for which accurate radial velocities are available in the catalogue by $\mathrm{Du}-$ rand et al. (1998), comprising 867 objects. This is a more complete sample, so that it is expected that the derived results can be extended to the observed population of $\mathrm{PN}$ in the Galaxy. In both samples, the number of nebulae used depends on the availability of the statistical distances. The actual numbers of objects from the Maciel (1984) and Stanghellini et al. (2008) distance scales are 195 and 170 for Sample 1 and 493 and 403 for Sample 2, respectively. We have then applied the approximation given by equation (4) for both samples, with the coefficients shown in Table 2, considering only the objects for which ages in the interval $0<t(\mathrm{Gyr})<14$ could be obtained.

\section{RESULTS AND DISCUSSION}

The main results for the age distribution of the CSPN are shown in Figures 1-4, where we have used the age parameter definitions given in Tables 3 and 4 for Methods 1 and 2, respectively. Figures 1 and 2 refer to Sample 1, while Figures 3 and 4 refer to Sample 2. It can be seen that the age distributions obtained by both methods are similar, in the sense that most objects have ages under 5 Gyr, with a strong peak at ages typically between 1 and 3 Gyr. The histograms of Figures 3-4 are summarized in Table 5, where the fraction of stars obtained by Method 1 (ages $t_{1}$ to $t_{8}$ ) and Method 2 (ages $t_{9}$ to $t_{16}$ ) are shown for three age bins, namely 0-3 Gyr, 3-6 Gyr, and $t>6$ Gyr. 

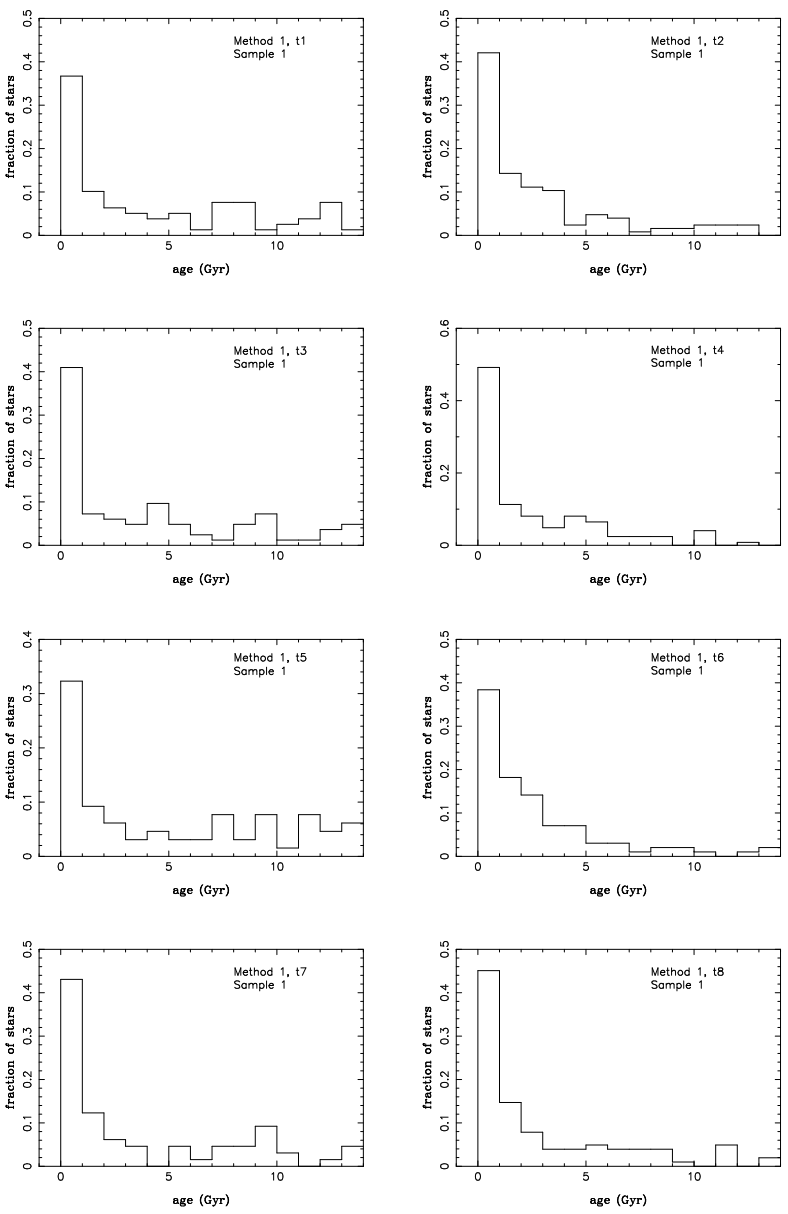

Fig. 1. Age distribution of CSPN, Method 1, Sample 1.

The similarity of the results of both methods is remarkable, especially considering that Method 2 is probably more accurate than Method 1. Method 2 consists of straightforward calculations of the velocities and velocity dispersions followed by an application of relatively accurate correlations involving the kinematics and ages of the objects considered. On the other hand, Method 1 is based on the assumption that the differences between the observed and predicted rotation velocities are essentially due to age effects. However, other processes may be important, such as deviations from the circular rotation, which is particularly important for nearby objects. According to Table 5, in all cases the vast majority of CSPN have ages under 3 Gyr. For Method 1 the total fraction of objects with $t \leq 3$ Gyr is 50 $70 \%$, while for Method 2 this fraction is somewhat higher, 70-90\%. It is unlikely that this is a result from bias in the samples, as the results for the larger Sample 2 are essentially the same as in the smaller Sample 1. It should be pointed out that the latter,
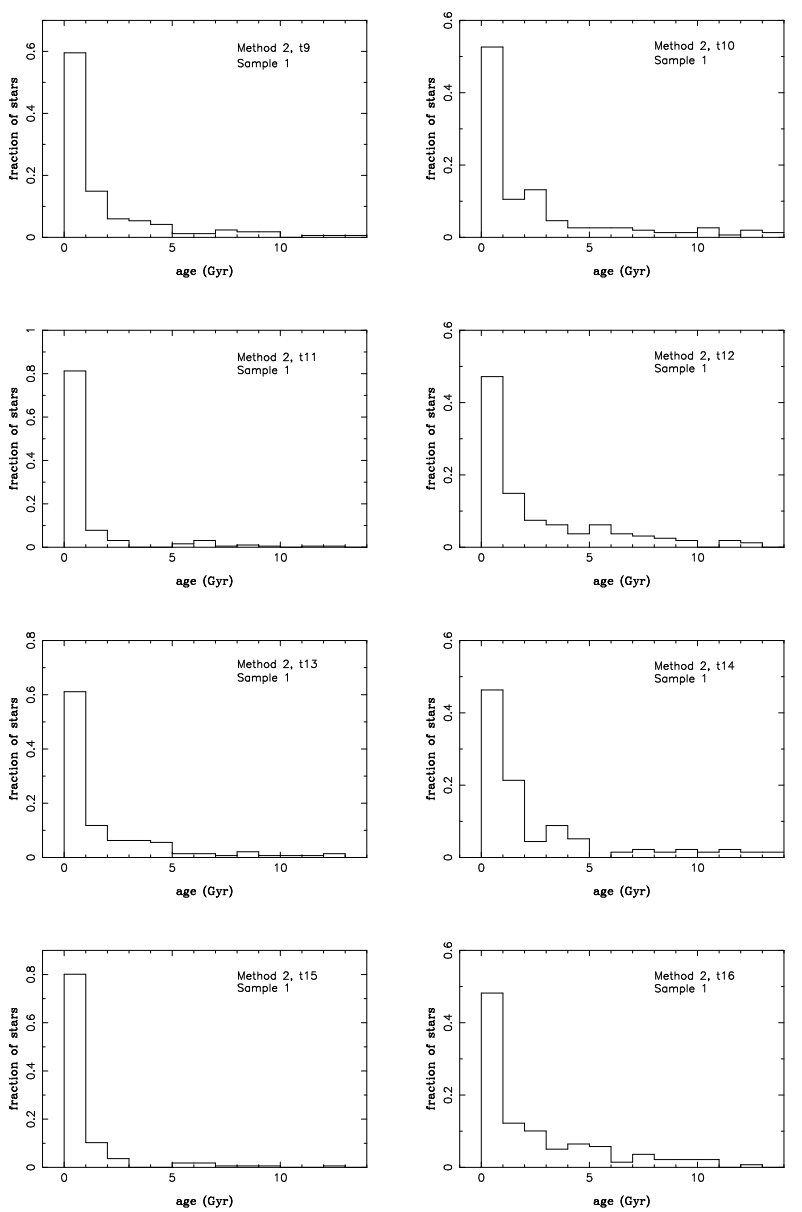

Fig. 2. Age distribution of CSPN, Method 2, Sample 1.

albeit smaller, includes only well studied nebulae, for which all individual parameters (distances, velocities, abundances) are better determined.

Also, there are no significant differences in the results using the different velocity components $U, V$, $W$, and $T$. For Method 1, the distributions using the $V$ velocity component are essentially the same as those using the total velocity, for both distance scales and samples. For Method 2, the distributions are slightly more concentrated in the first few age bins for the $W$ component, compared with the distributions for the $U$ and $V$ components and the total velocity, again for both distance scales and samples. Since the $W$ component is more clearly associated with the disk heating, essentially caused by age effects, the corresponding distributions are probably more accurate.

Similar remarks can be made regarding the adopted values for the proper motions. As mentioned at the end of $\S 2$, the results shown here assume that $\mu_{\alpha} \simeq \mu_{\delta} \simeq 0$. Adopting nonzero values 

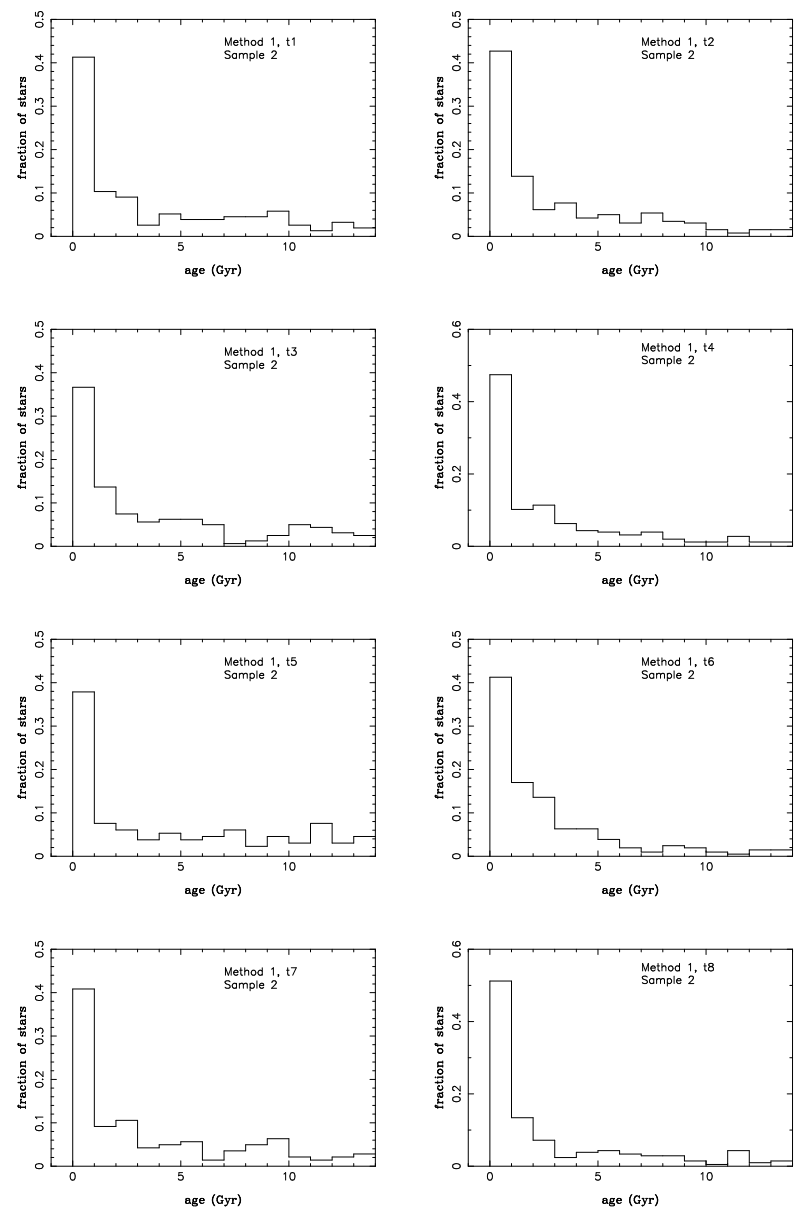

Fig. 3. Age distribution of CSPN, Method 1, Sample 2.

for these quantities $\left(\mu_{\alpha} \simeq \mu_{\delta} \neq 0\right)$, either the $V$ or $W$ component distributions become slightly less concentrated at the first few age bins, but most objects still have ages under about 4 Gyr. Again, the application of the SVD technique could be useful to confirm these results.

The uncertainties in the distances of the Milky Way PN are difficult to estimate, but the procedure adopted here ensures that the obtained age distributions are not particularly affected by the individual distances of the objects in the samples. As mentioned in $\S 2$, we have adopted two very different statistical scales, and the derived age distrbutions are essentially the same in both cases. The individual distances may depend on the particular scale, but the results shown in Figures 1-4 and in Table 5 do not depend on the choice of the distance scale. This can be seen by comparing the results for the timescales $t_{1}-t_{4}$ with those for $t_{5}-t_{8}$, or the results for $t_{9}-t_{12}$ with those for $t_{13}-t_{16}$.
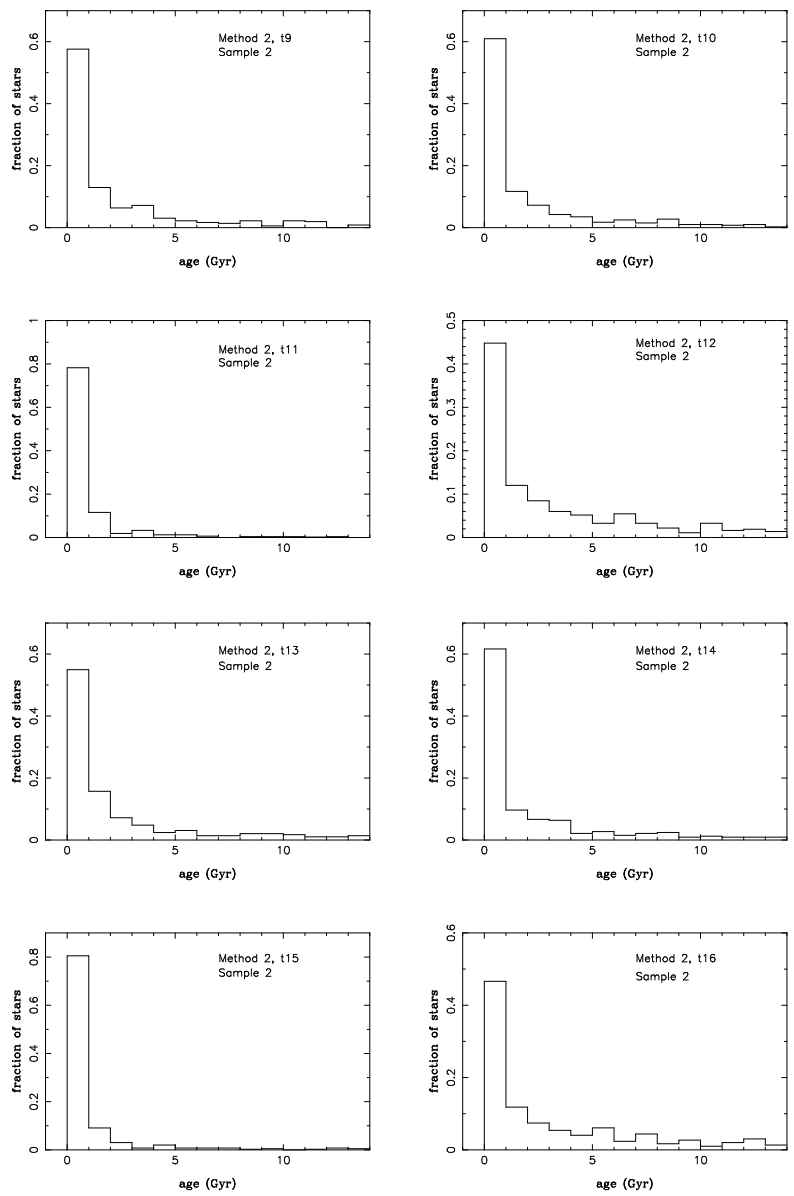

Fig. 4. Age distribution of CSPN, Method 2, Sample 2.

The uncertainties in the radial velocities also do not seem to have an important effect on the age distributions. In the catalogue by Durand et al. (1998), most objects $(\sim 90 \%)$ have uncertainties smaller than $20 \mathrm{~km} \mathrm{~s}^{-1}$, and many objects have much lower uncertainties. Concerning Method 1, from Maciel \& Lago (2005), the average rms deviation in the rotation velocity is about $50 \mathrm{~km} \mathrm{~s}^{-1}$ for PN, which can be compared with the values of about $20 \mathrm{~km} \mathrm{~s}^{-1}$ for HII regions (see also Clemens 1985, and Maciel \& Dutra 1992).

Probably the main uncertainty of the age distributions is due to the calibration between the stellar ages and the velocity dispersions, given by equation (4), which affects both Method 1 and 2. From the Geneva-Copenhagen Survey, this relation has a dispersion of about $20 \mathrm{~km} \mathrm{~s}^{-1}$ in average, which corresponds roughly to an age uncertainty of about $25 \%$, amounting to less than $1.2 \mathrm{Gyr}$ for the objects of Figures 1-4. Therefore, the uncertainties 
TABLE 5

FRACTION OF STARS IN THREE AGE INTERVALS

\begin{tabular}{ccccc}
\hline & $\Delta t$ (Gyr) & $0-3$ & $3-6$ & $>6$ \\
\hline Method 1 & $t_{1}$ & 0.57 & 0.13 & 0.30 \\
& $t_{2}$ & 0.62 & 0.18 & 0.20 \\
& $t_{3}$ & 0.57 & 0.19 & 0.24 \\
& $t_{4}$ & 0.67 & 0.18 & 0.16 \\
& $t_{5}$ & 0.51 & 0.13 & 0.36 \\
& $t_{6}$ & 0.71 & 0.17 & 0.12 \\
& $t_{7}$ & 0.61 & 0.15 & 0.24 \\
& $t_{8}$ & 0.71 & 0.11 & 0.18 \\
& & & & \\
Method 2 & $t_{9}$ & 0.76 & 0.12 & 0.12 \\
& $t_{10}$ & 0.79 & 0.10 & 0.11 \\
& $t_{11}$ & 0.92 & 0.04 & 0.04 \\
& $t_{12}$ & 0.77 & 0.18 & 0.05 \\
& $t_{13}$ & 0.78 & 0.10 & 0.12 \\
& $t_{14}$ & 0.78 & 0.11 & 0.11 \\
& $t_{15}$ & 0.93 & 0.03 & 0.04 \\
& $t_{16}$ & 0.76 & 0.18 & 0.06 \\
\hline
\end{tabular}

of the present method are comparable and probably smaller than in the case of the methods based on age-metallicity relations considered by Maciel et al. (2010).

The results for Sample 2 are not essentially different from those of Sample 1, so that a direct comparison can be made with the results by Maciel et al. (2010). The results of both investigations are similar, even though the present methods are completely independent of the metallicity-based methods used by Maciel et al. (2010). The main difference is that the kinematic methods used in the present investigation suggest somewhat lower ages for the CSPN in our samples. In this respect, these results fit nicely with the probability distribution for the progenitors of the CSPN according to Maciel et al. (2010, cf.
Figure 7, dashed line). In this case the well known relation between the main sequence mass and the stellar ages by Bahcall \& Piran (1983) was adopted, taking $t=10 \mathrm{Gyr}$ for $1 M_{\odot}$ stars on the main sequence. Taking into account the uncertainties of the methods, which are typically in the range 1-2 Gyr, this case was considered as the most realistic, so that it is reassuring that the kinematic methods produce similar results.

We thank Dr. R. Branham, Jr., for some interesting comments on an earlier version of this paper. This work was partly supported by FAPESP and CNPq.

\section{REFERENCES}

Bahcall, J. N., \& Piran, T. 1983, ApJ, 267, L77

Boesgaard, A. M., \& Tripicco, M. J. 1986, ApJ, 303, 724

Branham, R. L., Jr. 2010, MNRAS, 409, 1269

Cahn, J. H., Kaler, J. B., \& Stanghellini, L. 1992, A\&AS, 94, 399

Clemens, D. C. 1985, ApJ, 295, 422

Daub, C. T. 1982, ApJ, 260, 612

Durand, S., Acker, A., \& Zijlstra, A. 1998, A\&S, 132, 13

Holmberg, J., Nordström, B., \& Andersen, J. 2007, A\&A, 475,519

.2009, A\&A, 501, 941

Maciel, W. J. 1984, A\&AS, 55, 253

Maciel, W. J., Costa, R. D. D., \& Idiart, T. E. P. 2010, A\&A, 512, A19

Maciel, W. J., Costa, R. D. D., \& Uchida, M. M. M. 2003, A\&A, 397, 667

Maciel, W. J., \& Dutra, C. M. 1992, A\&A, 262, 271

Maciel, W. J., \& Lago, L. G. 2005, RevMexAA, 41, 383

Maciel, W. J., Lago, L. G., \& Costa, R. D. D. 2005, A\&A, 433, 127 2006, A\&A, 453, 587

Nordström, B., et al. 2004, A\&A, 418, 989

Perryman, M. 2009, Astronomical Applications of Astrometry: Ten Years of Exploitation of the Hipparcos Satellite Data (Cambridge: Cambridge Univ. Press)

Reid, M. 2010, http://www.cfa.harvard.edu/events/ 2010/dyn

Stanghellini, L., Shaw, R. A., \& Villaver, E. 2008, ApJ, 689,194

W. J. Maciel, T. S. Rodrigues and R. D. D. Costa: Instituto de Astronomia, Geofísica e Ciências Atmosféricas, Universidade de São Paulo, Rua do Matão 1226, CEP 05508-090, São Paulo SP, Brazil (maciel, tsrodrigues, roberto@astro.iag.usp.br). 